Florida International University FIU Digital Commons

\title{
The Use of Elemental Databases in Forensic Science: Studies on Vehicle Glass Interpretation and Milk Powder Provenancing
}

Tricia Marie Hoffman

thoff010@fiu.edu

DOI: $10.25148 /$ etd.FIDC006821

Follow this and additional works at: https://digitalcommons.fiu.edu/etd

Part of the Analytical Chemistry Commons, Food Chemistry Commons, and the Multivariate Analysis Commons

\section{Recommended Citation}

Hoffman, Tricia Marie, "The Use of Elemental Databases in Forensic Science: Studies on Vehicle Glass Interpretation and Milk Powder Provenancing" (2018). FIU Electronic Theses and Dissertations. 3812.

https://digitalcommons.fiu.edu/etd/3812 


\title{
FLORIDA INTERNATIONAL UNIVERSITY
}

Miami, Florida

\section{THE USE OF ELEMENTAL DATABASES IN FORENSIC SCIENCE: STUDIES ON} VEHICLE GLASS INTERPRETATION AND MILK POWDER PROVENANCING

\author{
A dissertation submitted in partial fulfillment of \\ the requirements for the degree of \\ DOCTOR OF PHILOSOPHY \\ in \\ CHEMISTRY \\ by
}

Tricia Marie Hoffman 
To: Dean Michael R. Heithaus

College of Arts, Sciences and Education

This dissertation, written by Tricia Marie Hoffman, and entitled The Use of Elemental Databases in Forensic Science: Studies on Vehicle Glass Interpretation and Milk Powder Provenancing, having been approved in respect to style and intellectual content, is referred to you for judgment.

We have read this dissertation and recommend that it be approved.

$\begin{array}{r}\text { Yong Cai } \\ \hline \text { Anthony DeCaprio } \\ \hline \text { Katma Huffman } \\ \hline \text { Jose Almirall, Major Professor }\end{array}$

Date of Defense: May 30, 2018

The dissertation of Tricia Marie Hoffman is approved.

Dean Michael R. Heithaus College of Arts, Sciences and Education

Andrés G. Gil

Vice President for Research and Economic Development and Dean of the University Graduate School

Florida International University, 2018 


\section{ACKNOWLEDGMENTS}

My sincerest gratitude goes to all my professors and friends. It is impossible to have completed this project on my own. I would like to thank you all for seeing that I had potential and pushing me to excel even when I doubted myself.

First, I'd like to acknowledge and thank Dr. José Almirall for his leadership and encouragement. He gave me the opportunity to become a part of his research group, which became my family and strongest support system while at FIU. I would also like to thank my dissertation committee Dr. Yong Cai, Dr. Anthony DeCaprio, Dr. Fatma Huffman, and Dr. Konstantinos Kavallieratos for their time and guidance.

I'd like to also acknowledge those that helped with the milk powder project. The members of the IAEA/FAO cooperation are acknowledged for their assistance in providing samples and analytical data. Also, Dr. Nives Ogrinc, Dr. Radojko Jaćimović, and Marta Jagodic are acknowledged for their assistance and guidance throughout the Slovenia - American cooperation.

I'd also like to acknowledge the Glass Interpretation Working Group (GIWG) and Vidia for their assistance with the glass project. Without them, this project would not have been possible.

Many thanks to Dr. Tatiana Trejos, Dr. Sarah Jantzi, and Pupi Tomassini for their guidance, time, and support both personally and professionally. I would also like to thank Ruthie for all her help when my Excel kept crashing.

I would like to acknowledge the Nuclear Regulatory Commission (NRC-HQ-8414-G-0040) for the $\mathrm{PhD}$ fellowship, research funds, and travel funds; the National Institute of Justice (2015-DN-BX-K049), which provided funding for the glass project; 
and the Graduate and Professional Student Committee for providing travel funds for research and conference travel throughout my graduate school career.

Last but not least, I would like to thank my families for motivating me to be the best that I could be and my best friend, Jonathan Coppella, for sticking by my side and giving me the confidence I needed to push through the difficult times. 


\section{ABSTRACT OF THE DISSERTATION}

THE USE OF ELEMENTAL DATABASES IN FORENSIC SCIENCE: STUDIES ON VEHICLE GLASS INTERPRETATION AND MILK POWDER PROVENANCING

by

Tricia Marie Hoffman

Florida International University, 2018

Miami, Florida

Professor Jose Almirall, Major Professor

The first study focuses on the development of a laser based method for the elemental analysis of solid milk powder. Milk powder samples originating from five different countries were analyzed to determine any geographic differences. A LA-ICPMS method was developed and compared to $k_{0}$-INAA for several milk samples as well as a reference sample. Precision of $10 \%$ RSD or better and a bias of $10 \%$ was achieved for both techniques for most elements with LA-ICP-MS producing lower limits of detection ( $1 \mathrm{ppm}$ ) for strontium. The comparison of LA-ICP-MS to $k_{0}$-INAA showed overlap of the $95 \%$ confidence intervals for all comparison samples. The data for 68 authentic milk powder samples representing five different countries (Argentina, Russia, Singapore, Slovenia, and the United States) were collected and used as a preliminary database. Principle component analysis (PCA) shows different groupings for the United States, Argentina, Singapore, and Slovenia. However the large number and geographic distribution of samples from Russia were not able to be distinguished from the samples from the United States and Slovenia. 
The second study focuses on the use of trace element databases for the objective interpretation of forensic glass evidence. Ten laboratories conducting analysis of glass participated in three inter-laboratory exercises. The aims of these exercises were to evaluate the use of a standard method for the analysis and comparison of glass evidence and to investigate different statistical approaches for interpreting results. Elemental analysis was performed on 420 vehicle windshield samples collected from 210 different vehicles representing manufacturing dates between 2004-2017 and 26 vehicle manufacturers. Using a variation of a previously reported comparison criterion for comparing samples to a database, the false exclusion rate and false inclusion rate for the new vehicle database were calculated to be $1.9 \%$ and $0.1 \%$ respectively. This criterion was used to calculate the frequency of an elemental profile for the case scenarios distributed as part of the inter-laboratory exercises. Similarities were observed between labs that calibrated their data the same way, thus showing it is possible for labs to use a central database. 


\section{TABLE OF CONTENTS}

CHAPTER

CHAPTER 1. Introduction to Milk Provenancing and Background on Milk

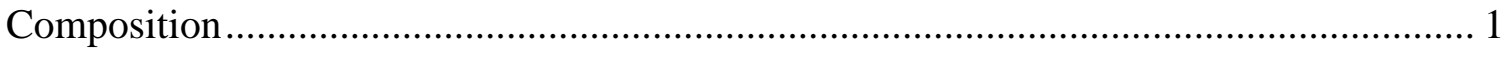

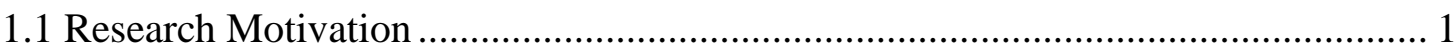

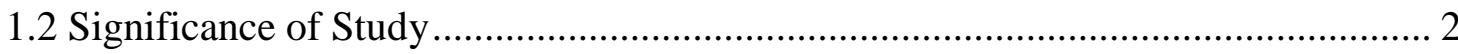

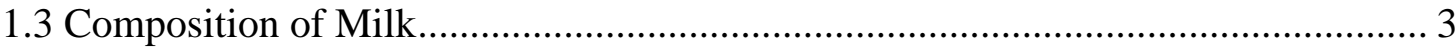

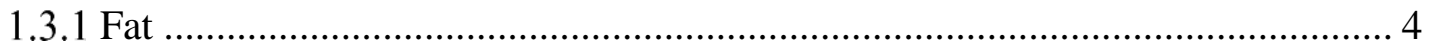

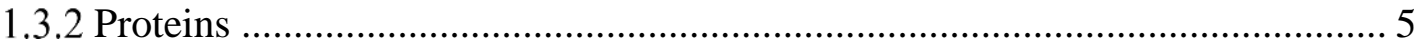

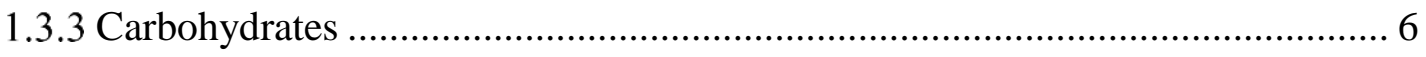

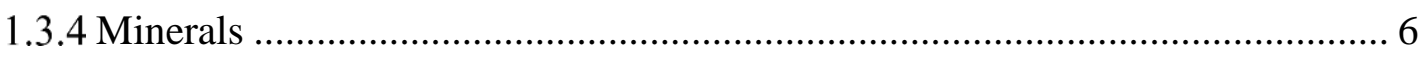

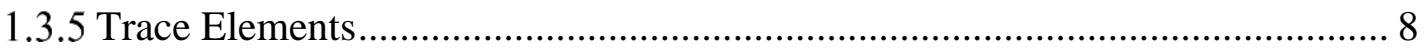

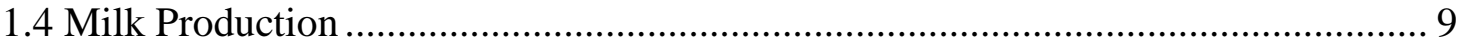

1.4.1 Collection and Storage ........................................................................ 9

1.4.2 Standardization of Fat Content .................................................................. 10

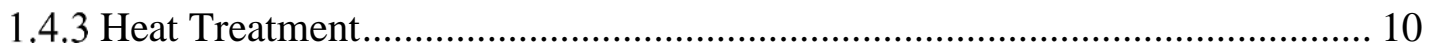

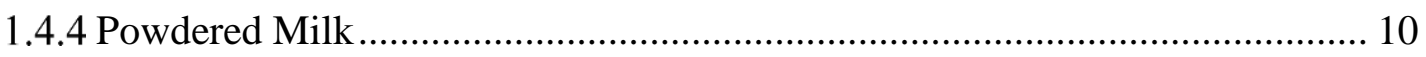

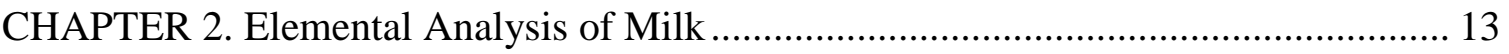

2.1 Principles of Analytical Techniques .................................................................. 14

2.1.1 $k_{0}$-Instrumental Neutron Activation Analysis .................................................. 14

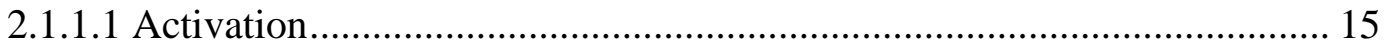

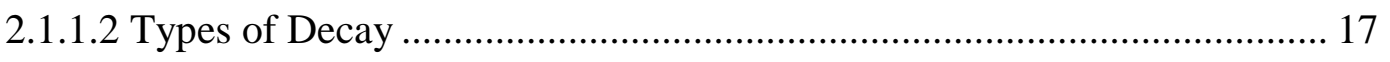

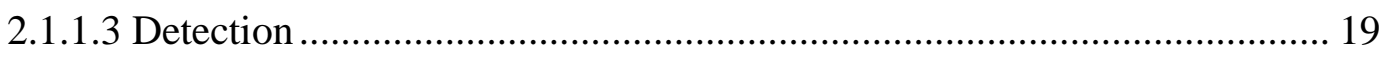

2.1.1.4 Determining Concentration with the $k_{\mathrm{o}}$ Method .................................... 21

2.2 Materials and Methods for Analysis of Milk ........................................................ 22

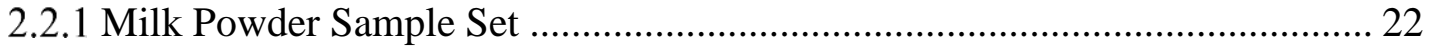

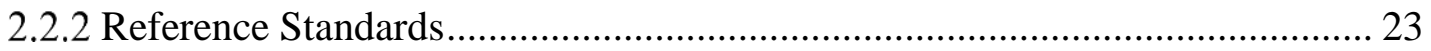

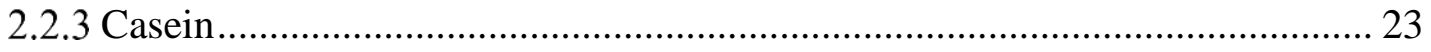

2.2.4 Commercial Milk Powder...................................................................... 24

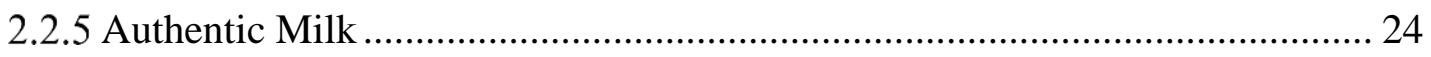

2.2.6 Authentic Data ……………………………………............................... 25 


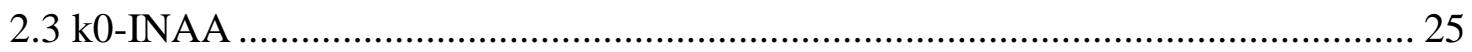

2.3.1 Preparation of Milk Samples for k0-INAA ……………………………....... 25

2.3.2 k0-INAA Instrument Parameters ................................................................ 26

2.3.3 k0-INAA Data Analysis ........................................................................ 28

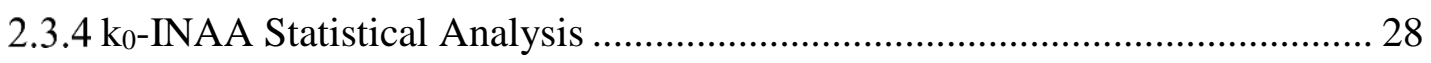

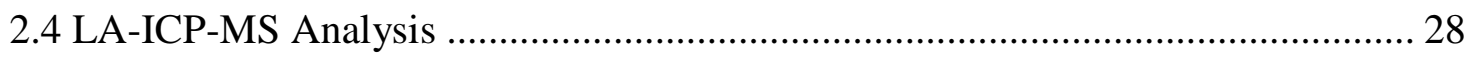

2.4.1 Preparation of Milk Samples for LA-ICP-MS ............................................... 28

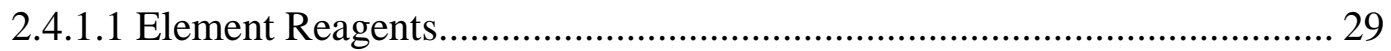

2.4.1.2 Sample Preparation for Pelleting Method \#1 .......................................... 29

2.4.1.3 Sample Preparation for Pelleting Method \#2 …………………................. 30

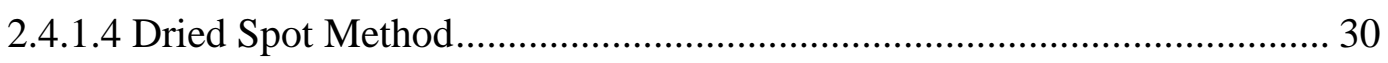

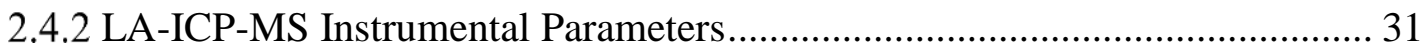

2.4.3 LA-ICP-MS Data Analysis ....................................................................... 33

2.4.4 LA-ICP-MS Statistical Analysis ................................................................. 35

CHAPTER 3. Optimization of Sample Preparation for LA-ICP-MS ................................ 38

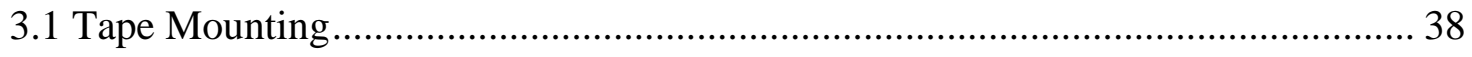

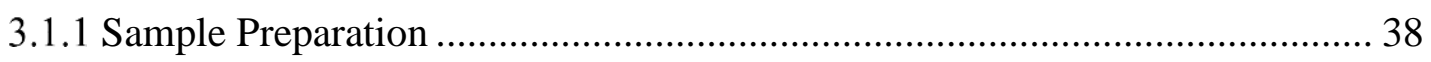

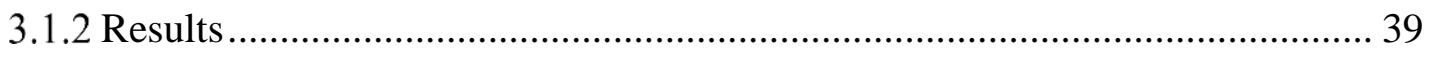

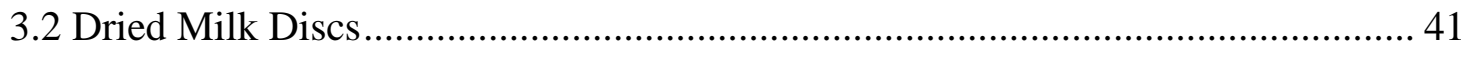

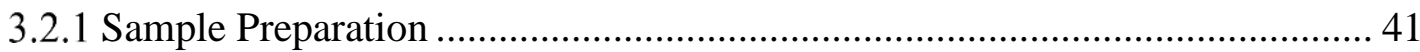

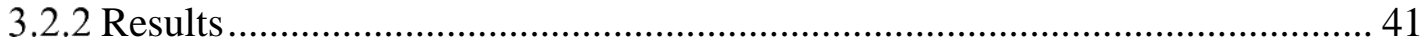

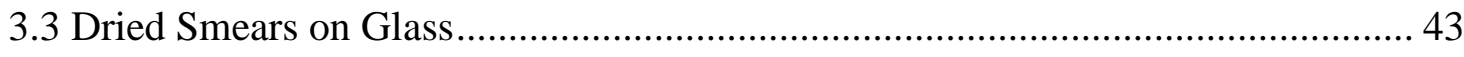

3.3.1 Sample Preparation ............................................................................. 43

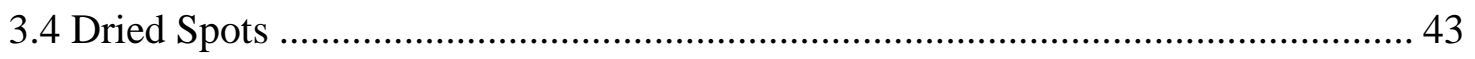

3.4.1 Selecting a Substrate and Volume Size........................................................... 43

3.4.2 External Calibration Curve ......................................................................... 46

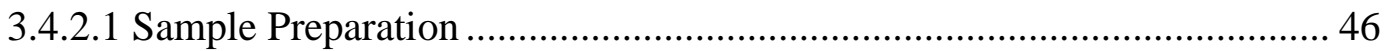

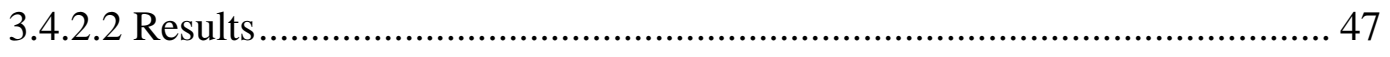

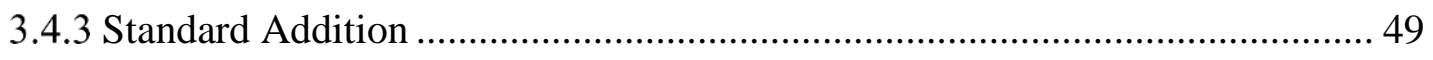

3.4.3.1 Sample Preparation ............................................................................. 49

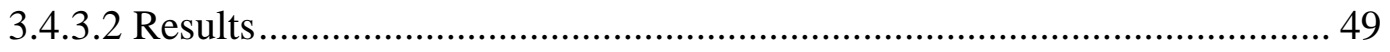


3.4.4 External Calibration Curve using IAEA-153 .......................................... 51

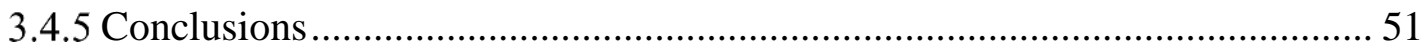

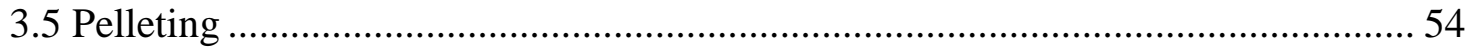

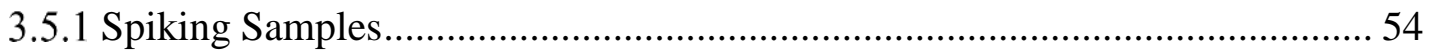

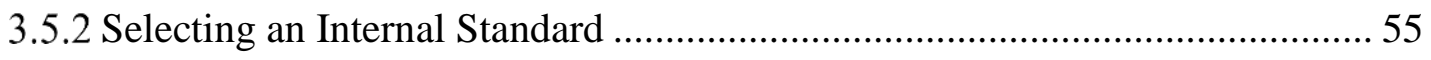

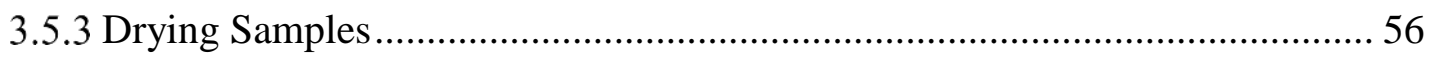

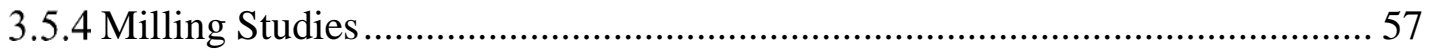

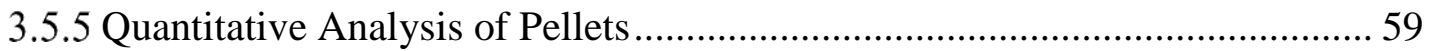

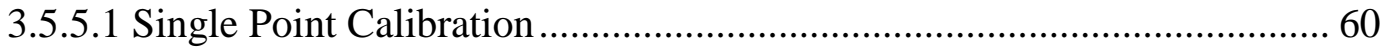

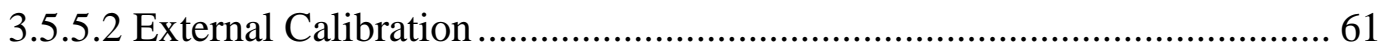

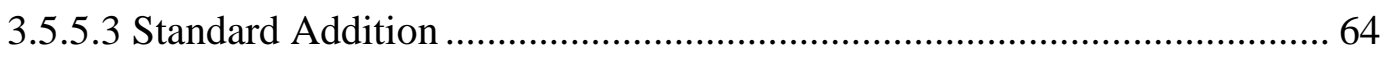

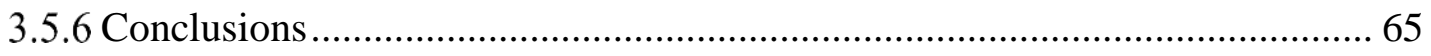

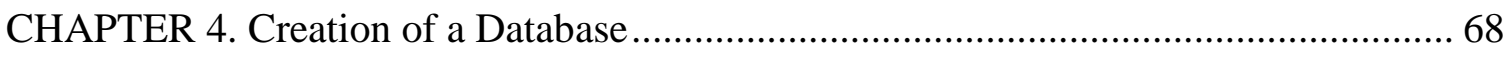

4.1 Collection of Data for Authentic Samples ......................................................... 71

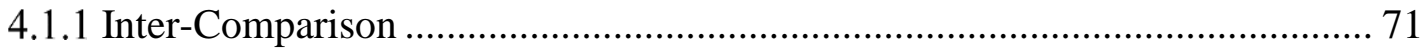

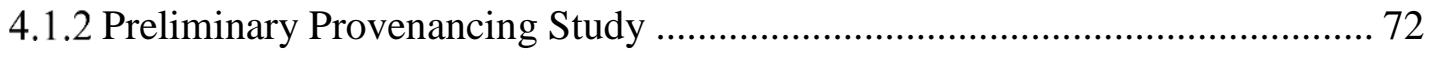

CHAPTER 5. Analysis of Milk Powder Conclusion.................................................. 78

CHAPTER 6. Introduction to Forensic Analysis of Glass ....................................... 81

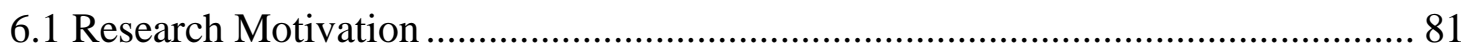

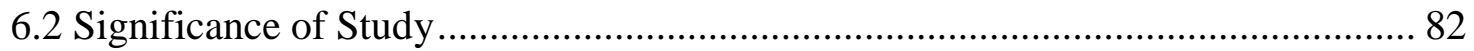

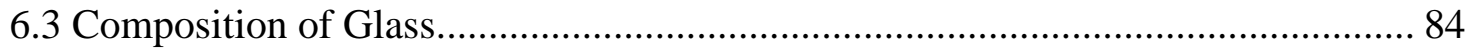

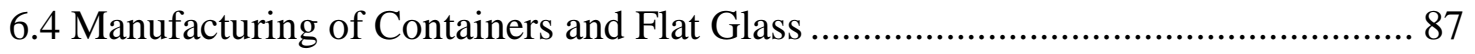

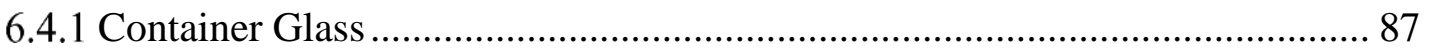

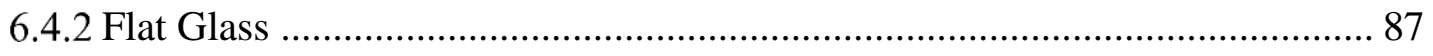

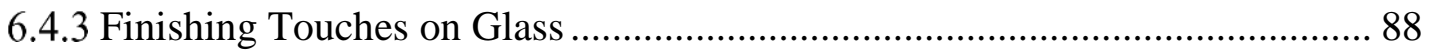

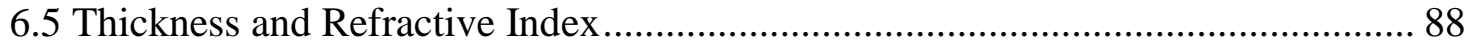

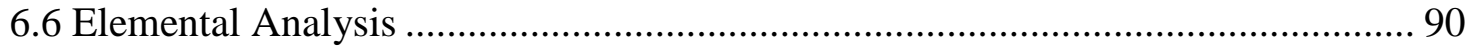

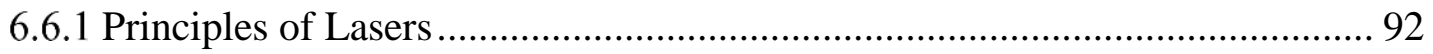

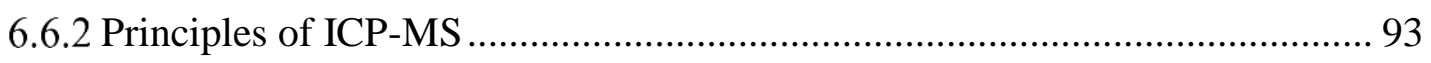

6.7 Comparison Criteria for LA-ICP-MS measurements ...................................... 97

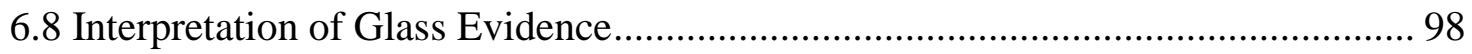




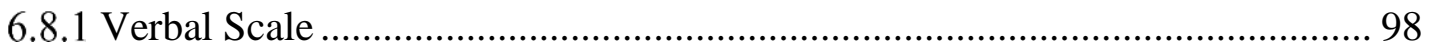

6.8.2 Random Match Probability ........................................................................ 100

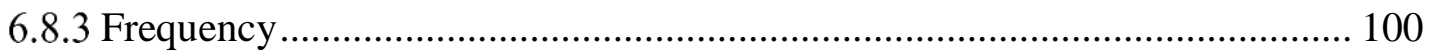

CHAPTER 7. Creating the M\&M Salvage Yard Glass Database ................................... 101

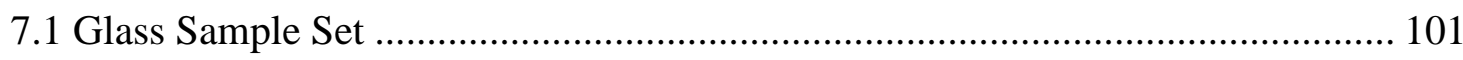

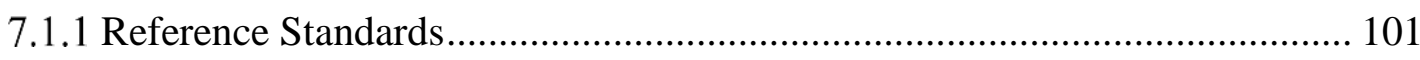

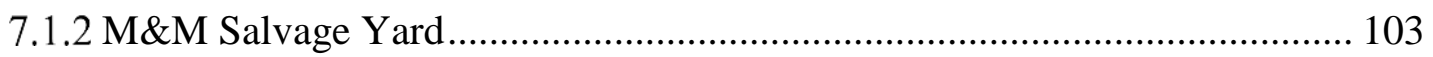

7.2 Preparation of Glass Samples for LA-ICP-MS ..................................................... 104

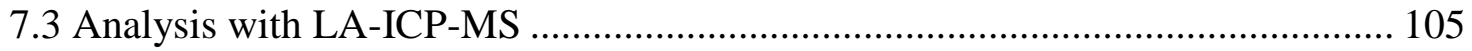

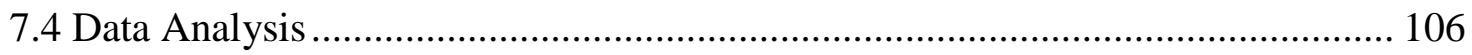

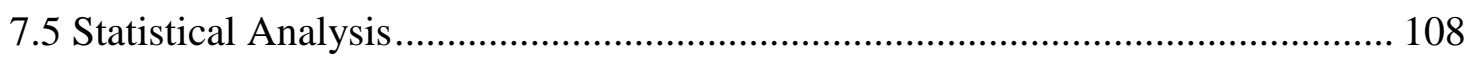

7.5.1 FGS1 and NIST1831 Control Charts ....................................................... 108

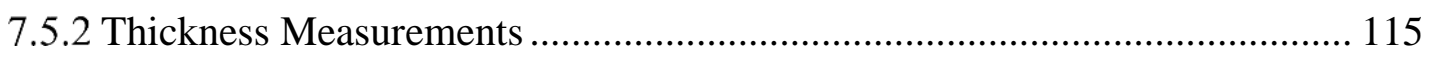

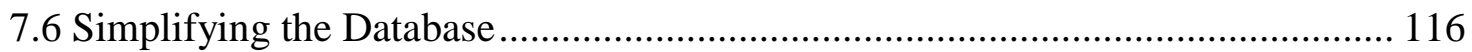

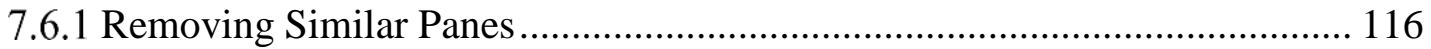

7.6.2 Limiting the Element Menu ......................................................................... 117

7.7 Determining Random Match Probability and Frequency Comparison Criteria ... 118

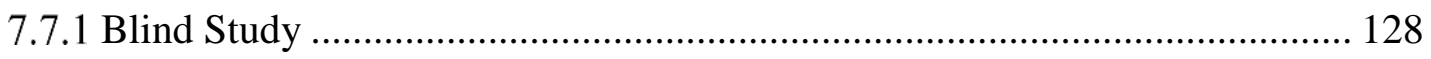

7.8 Discrimination Potential ................................................................................ 130

CHAPTER 8. Collection and Evaluation of Glass Databases ........................................ 133

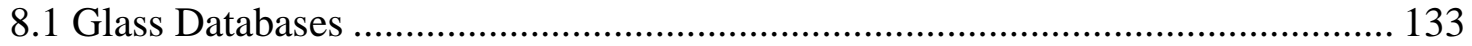

8.2 Random Match Probability ……………………….............................................. 133

CHAPTER 9. Equivalence Test vs. ASTM E2927 Match Criterion ............................... 137

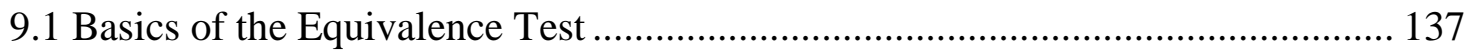

9.2 Evaluating the Equivalence Test using EAWG Inter-Laboratory Studies ........... 140

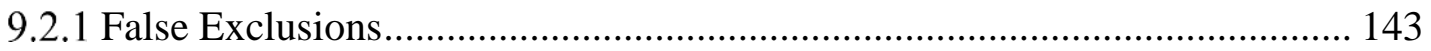

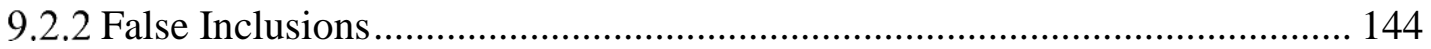

9.3 ASTM E2927 Error Rates for the M\&M Glass Database ………....................... 145

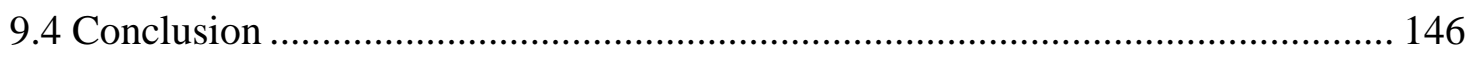

CHAPTER 10. Glass Interpretation Working Group Inter-Laboratory Studies............. 147 


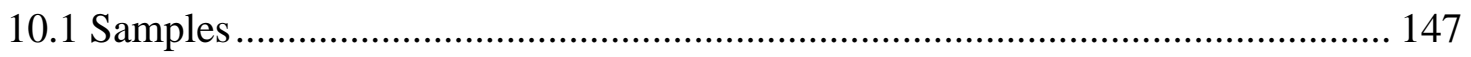

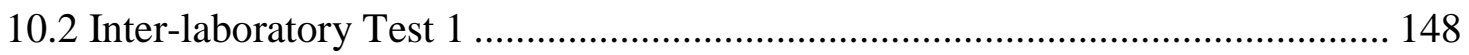

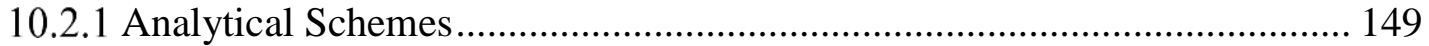

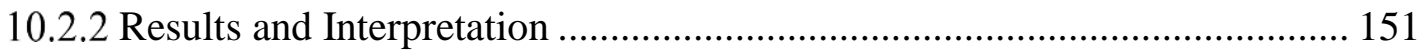

10.2.3 Interpretation for First Inter-Laboratory Test ............................................. 156

10.2.4 Frequency of Occurrence for the First Inter-Laboratory Exercise............... 157

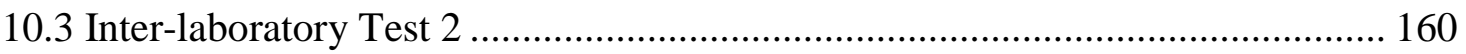

10.3.1 Evaluation of ASTM E2927-17e1 as a Method for Glass Comparisons ..... 161

10.3.2 Interpretation for Second Inter-Laboratory Exercise .................................. 162

10.3.3 Frequency for Second Inter-Laboratory Exercise ........................................ 163

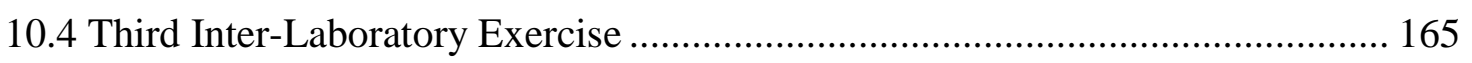

10.4.1 Evaluation of ASTM E2927 ................................................................. 167

10.4.2 Interpretation of the Third Inter-Laboratory Exercise Results...................... 168

10.4.3 Frequency for Third Inter-Laboratory Test................................................. 168

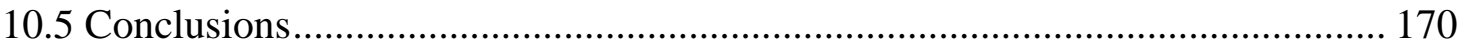

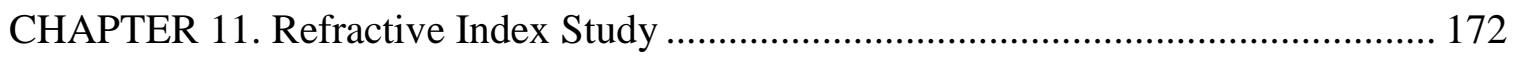

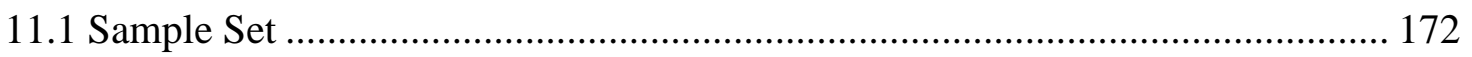

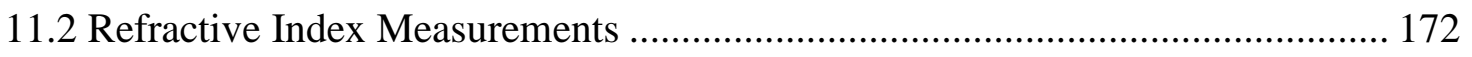

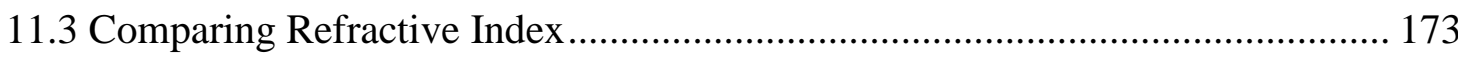

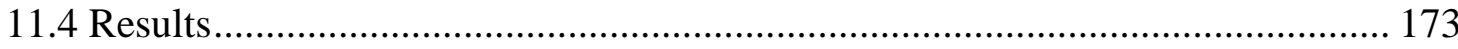

CHAPTER 12. Analysis \& Interpretation of Glass Conclusions ................................... 176

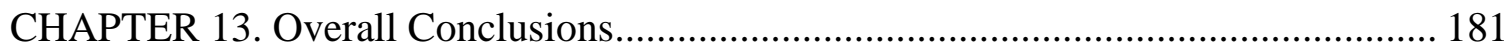

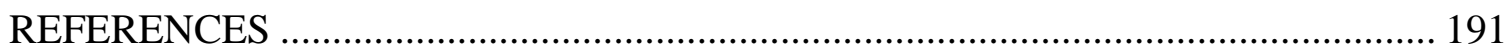

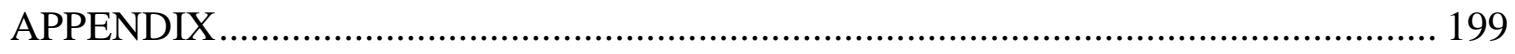

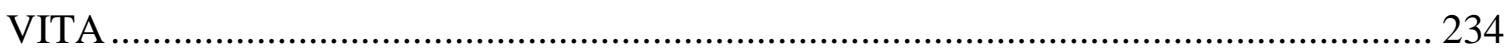


Table 1 - Composition of main constituents in milk.......................................................... 3

Table 2 - Concentrations $(\mathrm{mg} / 100 \mathrm{~g})$ of some metals in different types of milk [7] ........... 8

Table 3 - Concentration of elements of interest in casein determined by solution ICP-

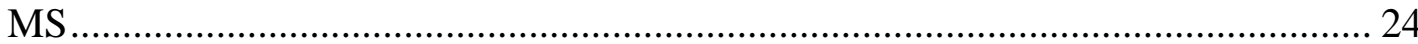

Table 4 - Laser parameters for the pelleting and dried spot sample preparations ............. 33

Table 5 - Results for IAEA-153 using the dried disc method with single point calibration ...................................................................................................... 42

Table 6 - Shown are the calculated concentration, uncertainty, and bias for IAEA153 using the different calibration strategies and the dried spot sample

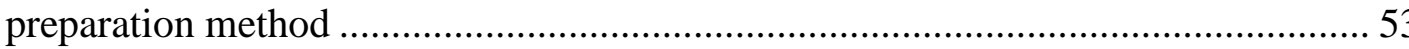

Table 7 - The concentration of each element added to the each of the calibration pellets; to prevent acid digestion of the milk powders, the element spikes were spread out so each standard received the same spike volume. The lowest calibration standard for one element might be the highest calibration standard for another.

Table 8 - Figures of merit for IAEA-153 using the two different pellet sample preparations and the different calibration strategies. The values in red are higher than the desired $\pm 10 \%$. The uncertainty for the standard addition and external calibration methods in the table are given as either $\mathrm{S}_{\mathrm{XE}}(\% \mathrm{RSD})$ or $\mathrm{S}_{\mathrm{X} 0}(\% \mathrm{RSD})$... 67

Table 9 - Comparison of the LA-ICP-MS method to k0-INAA. Reported are the 95\% confidence intervals for both techniques

Table 10 - Displayed are common coloring elements and the color each produces [49]

Table 11 - Glass compositions with values expressed as weight percent [49] .................. 86

Table 12 - Average values for the composition of float glass (\% element by weight)..... 91

Table 13 - LA-ICP-MS instrumentation used by laboratories located around the

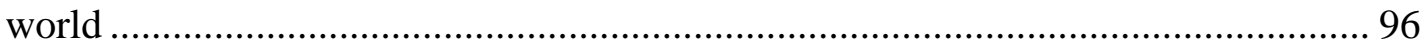

Table 14 - Concentrations (ppm) of elements in reference glasses ................................ 102

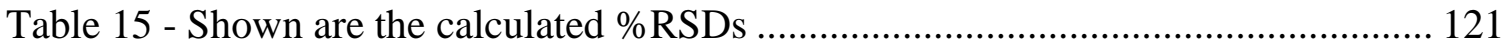


Table 16 - Duplicate samples and the days of analysis

Table 17 - The results for the duplicate samples with different comparison criteria. *This is the result of the ASTM E2927 comparison criterion. This criterion depends on which sample is selected as the Known sample, so the original data in the database was selected as the duplicate. The FRSD selected for further use in this study was FRSD (Adjusted FIU).

Table 18 - Comparing the blind samples to the database ........................................ 130

Table 19 - Description of each database donated to the FIU GIWG ............................ 135

Table 20 - False inclusion rates for the donated databases ...................................... 136

Table 21 - The samples used and the number of labs performing ICP-MS in EAWG.. 141

Table 22 - False inclusion and exclusion error rates for the different statistical test using the EAWG inter-laboratory test data. The first four tests listed in this table are the reported results found in [44]. The three equivalence tests are the results from this study. ...

Table 23 - False exclusion rate for M\&M Database .................................................. 146

Table 24 - GIWG inter-laboratory test samples ................................................. 148

Table 25 - Sample pairs that were found to be indistinguishable with LA-ICP-MS analysis used for refractive index study 


\section{LIST OF FIGURES}

FIGURE

PAGE

Figure 1 - Chemical structure of glycerol, a fatty acid, and triglyceride ........................... 4

Figure 2 - Chemical structure of an amino acid..................................................... 5

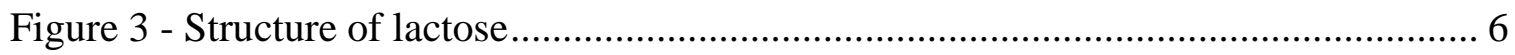

Figure 4 - The multi-stage evaporation process used to concentrate milk ..................... 11

Figure 5 - Neutron energy and flux found in reactors [34]; thermal neutrons $\left(10^{-1}-10\right.$ $\mathrm{eV})$ are used to induce fission and activate most elements................................. 17

Figure 6 - Decay scheme for ${ }^{60 \mathrm{~m}} \mathrm{Co}$ into ${ }^{60} \mathrm{Ni}$; during the decay two gamma rays are emitted with energy of $1173 \mathrm{keV}$ and $1332 \mathrm{keV}$............................................... 19

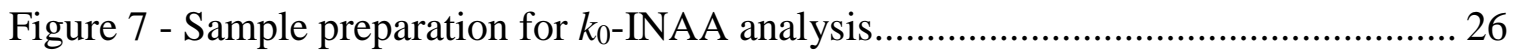

Figure 8 - Gamma spectrum of the gold comparator: 240 live seconds ........................ 27

Figure 9 - Gamma spectrum for IAEA-153: The first measurement is in red and was accumulated for 3600 live seconds. The second measurement is shown in blue and was accumulated for 60000 live seconds. The third measurement is in green and was accumulated for 52900 live seconds.

Figure 10 - Dried spot method before and after sitting overnight

Figure 11 - The different regions of the transient signal (counts vs time) for spot analysis on pellets

Figure 12 - The different regions for the transient signal (counts vs time) for radial line scan of the dried spots; the counts for the elements are not consistent over the entire spot. All elements of interest were found to be more concentrated on the edges of the dried spots.

Figure 13 - Transient signal for the tape sample preparation.

Figure 14 - Dried discs samples for IAEA-153 and IAEA-155 after addition of internal standard and $0.5 \mathrm{~mL}$ of water and drying overnight

Figure 15 - Different volume spikes on $16 \mathrm{~mm}$ dried spots ...................................... 45

Figure 16 - Different volume spikes on $6 \mathrm{~mm}$ dried spots ....................................... 45

Figure 17 - Comparison of dried spots on Whatman paper and polystyrene.................. 45 
Figure 18 - External calibration curves for $\mathrm{Na}, \mathrm{Mg}, \mathrm{Ca}, \mathrm{Rb}, \mathrm{Sr}$, and $\mathrm{Zn}$ using ICP-MS standards and the dried spot sample preparation method; the $\mathrm{y}$ error bars represent \pm 1 standard deviation. The red data points represent IAEA-153 and the measured counts per second. The $\mathrm{x}$ error bars on the red data points represent $\pm 1 \mathrm{~S}_{\mathrm{X} 0}$

Figure 19 - Standard addition curves for $\mathrm{Na}, \mathrm{Mg}, \mathrm{Ca}, \mathrm{Rb}, \mathrm{Sr}$, and $\mathrm{Zn}$ using IAEA153 and the dried spot sample preparation; the $\mathrm{y}$ error bars represent \pm 1 standard deviation. The $\mathrm{x}$ error bars are the $\mathrm{x}$-intercept represent $\pm 1 \mathrm{~S} \mathrm{XE}$.

Figure 20 - External calibration curves for $\mathrm{Na}, \mathrm{Mg}, \mathrm{Ca}, \mathrm{Rb}, \mathrm{Sr}$, and $\mathrm{Zn}$ created using IAEA-153 and the dried spot sample preparation; the green, purple, and orange data points represent three different milk powder samples. As can be seen these data points fall below or on the lower end of the calibration curve for most elements.

Figure 21 - LA-ICP-MS signal for pellets 56

Figure 22 - A. Sample adhering to mill jar because the frequency was too high and/or the length of milling was too long B. Clumps of milk powder that were not milled long enough at a high enough frequency.....

Figure 23 - A. is the transient signal for a commercial full cream milk powder sample with a homogenized internal standard B. is the transient signal for a commercial nonfat milk powder sample where the internal standard was not homogenized in the sample

Figure 24 - Craters created using the optimized laser parameters visualized using a Keyence 3D microscope

Figure 25 - External calibration curves for $\mathrm{Na}, \mathrm{Mg}, \mathrm{Ca}, \mathrm{K}, \mathrm{Rb}$, and $\mathrm{Sr}$ generated by diluting IAEA-155 with casein. The normalized, background subtracted CPS are plotted vs the concentration in the pellet. The $\mathrm{y}$ error bars represent \pm 1 standard deviation. The red data points represent the reported concentration for IAEA-153 at the CPS determined after data analysis. he $x$ error bars are the red data points represent $\pm 1 \mathrm{~S}_{\mathrm{x} 0}$

Figure 26 - Standard addition curves for $\mathrm{Na}, \mathrm{Mg}, \mathrm{Ca}, \mathrm{Sr}, \mathrm{Rb}$, and $\mathrm{Zn}$ in IAEA-153 using the pelleting method. The y error bars represent \pm 1 standard deviation. The $\mathrm{x}$ error bars represent $\pm 1 \mathrm{~S}_{\mathrm{XE}}$

Figure 27 - Inter-laboratory comparison of select elements in IAEA-153. Shown are the mean values determined by each lab for each element. Labs A and B produced results for $\mathrm{Zn}$ that fell outside \pm 3 standard deviations. Labs A, B, and $\mathrm{D}$ produced either inaccurate results or had high standard deviations for Ca. Both $\mathrm{Zn}$ and $\mathrm{Ca}$ were removed from the element menu. 
Figure 28 - The loading plots used for the PCAs.....

Figure 29 - A PCA plot depicting the authentic samples from each country. The pink stars represent the data from Argentina. The red circles represent the data from Russia. The green triangles represent the data from Singapore. The blue diamonds represent the data from Slovenia. The orange squares represent the data from the USA. The black Xs represent the data submitted from each lab for IAEA-153

Figure 30 - A PCA plot without Russia. The pink stars represent the data from Argentina. The green triangles represent the data from Singapore. The blue diamonds represent the data from Slovenia. The orange squares represent the data from the USA. The black Xs represent the data submitted from each lab for IAEA-153

Figure 31 - An example of a window marking found on the windshield of a vehicle.... 103

Figure 32 - Distribution of the years of vehicles sampled .......................................... 104

Figure 33 - Distribution of the makes of vehicles sampled .................................... 104

Figure 34 - Sample preparation for LA-ICP-MS ….............................................. 105

Figure 35 - Integration strategy used for glass................................................... 108

Figure 36 - Levey Jennings control charts for NIST1831 …................................... 110

Figure 37 - Correlation of elements in the M\&M database ..................................... 118

Figure 38 - Elements distinguishing duplicates using the max FIU FRSD ................. 124

Figure 39 - Examples of some duplicate pairs being distinguished using the maximum FRSDs of the M\&M database ................................................... 125

Figure 40 - Transient signal for Pb 208 in duplicate pair .......................................... 126

Figure 41 - Using the selected FRSD, the elements shown in the graph were found to incorrectly distinguish inter-day control samples. The $\mathrm{Sr}$ variation seen in NIST1831 was only seen in this glass. FGS1 and the duplicates appeared to have no problem with $\mathrm{Sr}$. $\mathrm{Pb}$ reproducibility was an issue for both control glasses and some of the duplicate pairs.

Figure 42 - Discrimination potential of each element. The grey columns represent the percent (\%) of comparisons that were distinguished by that element. The colored segmented columns represent how many comparisons were only distinguished by that element and what type of samples they were (same year, same make etc.) 
Figure 43 - Modified from [61], this figure depicts how the equivalence test decides if the sample means are practically equivalent.

Figure 44 - Equivalence test for Sr using the 4th inter-laboratory data (K1 vs Q2.1)... 144

Figure 45 - Transient signal of K in NIST612 and FGS2.

Figure 46 - Inter-lab comparison of NIST1831. Shown are each labs calculated mean \pm the standard deviation of the measurement for select elements

Figure 47 - Comparison of each lab's K1 outer to its duplicate in the database

Figure 48 - Comparison of each lab's K1 outer to sample 45 and sample 78 in the

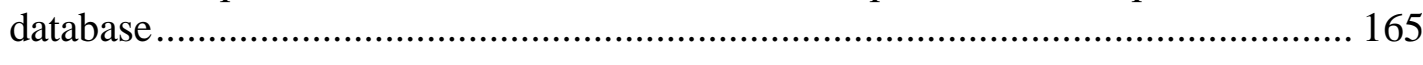

Figure 49 - ASTM E2927 comparison of K1 outer and Q2.1 …............................. 168

Figure 50 - Comparison of K1 outer to sample 78 and 21 in the database .................... 170 


\section{ABBREVIATIONS AND ACRONYMS}

ASTM E2927 Standard Test Method for Determination of Trace Elements in SodaLime Glass Samples Using Laser Ablation Inductively Coupled Plasma Mass Spectrometry for Forensic Comparisons

CPS counts per second

DS distinguished

EAWG Elemental Analysis Working Group

FGS 1 float glass standard 1

FGS 2 float glass standard 2

FRSD fixed relative standard deviation

GIWG Glass Interpretation Working Group

IAEA International Atomic Energy Agency

IAEA-153 milk powder reference standard

IAEA155 whey powder reference standard

ICP-MS inductively coupled plasma-mass spectrometry

ICP-OES inductively coupled plasma-optical emission spectroscopy

IN indistinguishable

K known glass sample

$k_{0}$-INAA Kay zero Instrumental Neutron Activation Analysis

LA laser ablation 


$\begin{array}{ll}\text { LA-ICP-MS } & \text { laser-ablation-inductively coupled plasma-mass spectrometry } \\ \text { LIBS } & \text { laser induced breakdown spectroscopy } \\ \text { LOD } & \text { limit of detection } \\ \text { NAA } & \text { neutron activation analysis } \\ \text { Nd:YAG } & \text { Neodymium Yttrium Aluminum Garnet } \\ \text { NIST612 } & \text { NIST glass standard } \\ \text { NIST1831 } & \text { NIST glass standard } \\ \text { NITECRIME } & \begin{array}{l}\text { Natural Isotopes and Trace Elements in Criminalistics and } \\ \text { Environmental Forensics } \\ \text { RSD }\end{array} \\ \text { questioned glass sample } \\ \text { RI } & \text { refractive index } \\ & \end{array}$




\section{CHAPTER 1. Introduction to Milk Provenancing and Background on Milk Composition}

\subsection{Research Motivation}

Food fraud, according to the U. S. Pharmacopeia Convention, is "a collective term that encompasses the deliberate substitution, addition, tampering or misrepresentation of food, food ingredients or food packaging, or false or misleading statements made about a product for economic gain" [1]. Food fraud can cause health problems and also be an issue of National Security if imported materials are being falsely labeled as coming from another country. A recent edition (Aug. 25, 2014) of Chemical and Engineering News featured a cover story on food fraud. This article listed milk as one of the top five adulterated foods [2].

The adulteration of milk, in particular milk powder generated from bovine milk, has occurred in a variety of ways. One of the major instances that sparked the interest in detecting and preventing adulteration in milk occurred in China. In 2008, six infants were killed and 30,000 others sickened by drinking melamine-laced baby formula. Since the structure of melamine contains 6 nitrogens per molecule, it was added to the milk powders to make it appear to contain a higher protein content [2]. Infant milk was not the

only contaminated product. It was revealed that virtually all Chinese-produced dairy products including ordinary milk, ice cream, and yogurt also contained melamine. Countries around the world announced bans on imports of products that contained Chinese milk, including bakery products and candies [3]. 
The European Commission has now begun trials of mandating that milk and milk products be labeled with the country of origin [4]. Because of the potential of falsifying information, the focus of this research is detecting instances of adulteration where companies mislabel a product as being a more expensive imported one [5]. Adulteration of imports/exports is an issue of national security. The International Atomic Energy Agency (IAEA) has joined the efforts of 13 countries in an attempt to characterize the organic and inorganic properties of milk powder. This research will aide in the creation of a database, which will allow for 1) the elemental characterization of milk powder and begin to develop a method to determine the provenance of milk powder samples.

\subsection{Significance of Study}

The present study focused on detecting fraudulently labeled milk. Given that milk is usually exported and imported as a powder, the development of laser-based methods for the analysis of solid milk powders was investigated. An LA-ICP-MS (laser ablationinductively coupled plasma-mass spectrometry) method was evaluated using the milk powder reference material IAEA-153 (Trace Elements in Milk Powder, International Atomic Energy Agency, Vienna, Austria). The results for 13 samples were compared with a $k_{0}$-INAA method that was previously developed to further test the accuracy of the LA-ICP-MS method [6]. This research then looked at combining elemental data from other laboratories. In order to assess the performance of each laboratory's methodology, each participant was asked to analyze IAEA-153. For this reference material, consistent results (within 95\% confidence) were obtained. To test the performance of a combined dataset, laboratories from five countries (Argentina, Russia, Singapore, Slovenia, and the 
United States) provided elemental data for authentic milk powder samples. Finally, using the data submitted from the participating laboratories, the discrimination capabilities of the LA-ICP-MS elemental menu was evaluated to determine if differences between countries could be detected.

\subsection{Composition of Milk}

There are many farm animals today that produce milk that is used for human consumption: bovine, sheep, goats, buffalo, and camels. Though similarities exist between all of these types of milk, this section will focus particularly on the composition of bovine milk. The components of milk can be broken down into organic (fats, proteins, and sugars) and inorganic (minerals). The typical concentration of these components in milk can be seen in Table 1. Since different breeds of cow produce milk with various compositions, the $\%$ composition is given as a range [7].

Table 1 - Composition of main constituents in milk

\begin{tabular}{|cc|}
\hline & \% Composition \\
\hline Water & $85.5-89.5$ \\
Total Solids & $10.5-14.5$ \\
Fat & $2.5-6.0$ \\
Proteins & $2.9-5.0$ \\
Lactose & $3.6-5.5$ \\
Minerals & $0.6-0.9$ \\
\hline
\end{tabular}


Since this research focuses on the elemental analysis of milk, the organic composition will only be briefly described since metals are often found attached to the major organic components. More time will be spent looking at metals present in milk and how they change over time.

\subsubsection{Fat}

Milk fat is mostly a combination of triglycerides. These compounds consist of three fatty acids attached to a glycerol backbone through ester bonds, Figure 1 . The $\mathrm{R}$ group on the fatty acid can vary in length and structure. When the R group contains only single bonds, the fats are called saturated (saturated with hydrogen). On the contrary, when a double bond is present the fats are called unsaturated. There are a variety of fatty acids in milk, but the main four, which account for over $50 \%$ of the fatty acid content are myristic, palmitic, stearic, and oleic acids [8].

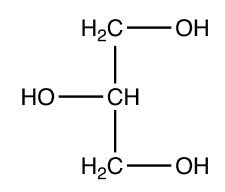

Glycerol<smiles>O=CO</smiles>

Fatty<smiles>[R]C(=O)OCC(COC([2H])=O)OC([2H])=O</smiles>

Triglyceride

Figure 1 - Chemical structure of glycerol, a fatty acid, and triglyceride

The fat component of milk is sometimes removed depending on the consumer's taste. Whole milk contains about $3.25 \%$ fat, but there is nonfat (skim) milk that has had most of the fat content removed, lowfat milk with only $1 \%$ fat, and reduced fat milk that contains $2 \%$ fat. 


\subsubsection{Proteins}

Proteins are made up of amino acids, which are compounds that contain an amino group and a carboxylic acid. The amino group and the carboxylic acid are attached to the same carbon. Amino acids differ from each other in the structure of an $\mathrm{R}$ side chain. Eight of the amino acids cannot be manufactured by the human body and therefore must be supplied through diet. These are called essential amino acids. Milk contains all of the essential amino acids.

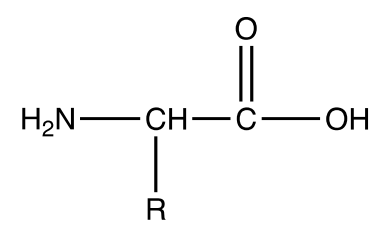

\section{Amino Acid}

\section{Figure 2 - Chemical structure of an amino acid}

There are hundreds are proteins in milk, but the two major ones are casein and milk serum proteins (whey). The properties of these proteins can be changed by altering heat and $\mathrm{pH}$, which allows for the manufacture of other dairy products such as cheese and yogurt [8]. Casein is involved with the stability of milk during heating and storage and also heavily involved in the formation of cheese. Whey protein contains many of the essential amino acids, and thus is sometimes separated from milk and used as a protein supplement for athletes and body builders. 


\subsubsection{Carbohydrates}

Carbohydrates are sugars. These compounds are the main source of energy. Carbohydrates can be classified as monosaccharaides (one sugar molecule), disaccharides (two sugar molecules), and polysaccharides (many sugars). The main carbohydrate in milk is lactose, which is a disaccharide made up of glucose and galactose, Figure 2. Like fat, lactose can also be removed from milk, and it is usually done so because some people cannot break down lactose.

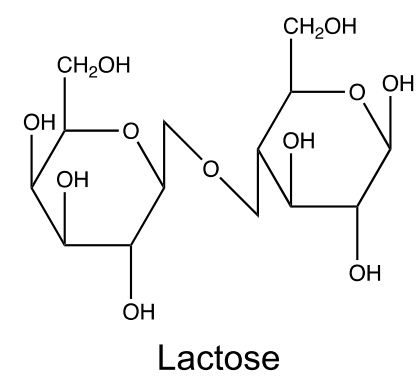

Figure 3 - Structure of lactose

\subsubsection{Minerals}

Minerals are naturally occurring inorganic substances. Mineral elements occur in milk and dairy products as inorganic ions and salts, as well as parts of organic molecules. These elements are the main focus of this research. The concentrations of these elements have been found to vary depending on how and if the milk was processed, the environment, the lactation cycle of the cow, and/or the breed of the cow $[9,10]$. Thus it may be possible to use the elemental composition of milk to determine if the country on a commercial product could be the origin of the product.

The major mineral elements present in milk are calcium $(\mathrm{Ca})$, phosphorus $(\mathrm{P})$, potassium $(\mathrm{K})$, sodium $(\mathrm{Na})$, magnesium $(\mathrm{Mg})$ and chlorine $(\mathrm{Cl})$. The concentrations of 
some of these mineral elements are reported to change because of a variety of factors. Micinski et al. reported that the concentration of $\mathrm{Ca}, \mathrm{K}, \mathrm{Na}, \mathrm{Mg}$, and $\mathrm{Zn}$ in milk changes with the age of the cow and $\mathrm{Ca} \mathrm{Mg}$, and $\mathrm{Zn}$ change during the lactation cycle [11]. Tsioulpas et al. also confirmed that the concentration of $\mathrm{Ca}$ was highest in colostrum and in the days immediately after giving birth [12]. Cerbulis et al. reported that the amount of these mineral elements also depends on the breed of cow and found that milk from Jersey cows differed from milk produced by Holstein cows [13]. Dandare et al. and Hermansen et al. also reported significant differences in the mineral content between different breeds of cows $[14,15]$. Other studies saw that these minerals can change with the health of the cow. For example $\mathrm{Na}$ and $\mathrm{Cl}$ were found to be more concentrated in milk from cows with udder infections. Table 2 shows some typical concentrations reported for elements of importance to this research in the various types of milk powders and pasteurized milk [16]. 
Table 2 - Concentrations (mg/100g) of some metals in different types of milk [7]

\begin{tabular}{|lcccccc|}
\hline & Ca & Mg & Na & K & Zn & Fe \\
\hline $\begin{array}{l}\text { Milk Powders } \\
\text { Whole Milk }\end{array}$ & 912 & 85 & 371 & 1330 & 3.34 & 0.470 \\
Skim Milk & 1257 & 110 & 535 & 1468 & 3.96 & 0.433 \\
$\begin{array}{l}\text { Liquid Milk } \\
\text { Whole Pasteurized Milk }\end{array}$ & 118 & 10.6 & 44.1 & 157 & 0.37 & 0.05 \\
Skim Milk & 125 & 11 & 42 & 156 & 0.42 & 0.03 \\
1\% Skim Milk & 119 & 11 & 44 & 150 & 0.42 & 0.03 \\
\hline
\end{tabular}

\subsubsection{Trace Elements}

Of the 20 essential minerals present in milk, 14 are present as trace elements: iron $(\mathrm{Fe})$, copper $(\mathrm{Cu})$, zinc $(\mathrm{Zn})$, manganese $(\mathrm{Mn})$, selenium $(\mathrm{Se})$, iodine $(\mathrm{I})$, chromium $(\mathrm{Cr})$, cobalt (Co), molybdenum (Mo), fluorine (F), arsenic (As), nickel (Ni), silicon ( $\mathrm{Si}$ ), and boron (B). Many other trace elements also occur in milk. However, they are not nutritionally important. These include elements such as lithium ( $\mathrm{Li})$, bromine $(\mathrm{Br})$, aluminum $(\mathrm{Al})$, strontium $(\mathrm{Sr})$, silver $(\mathrm{Ag})$, lead $(\mathrm{Pb})$, tin $(\mathrm{Sn})$, vanadium $(\mathrm{V})$, mercury $(\mathrm{Hg})$, cadmium $(\mathrm{Cd})$, rubidium $(\mathrm{Rb})$, and cesium $(\mathrm{Cs})$.

Some metals are used by cells to perform functions necessary for survival. Other metals such as lead $(\mathrm{Pb})$, and cadmium $(\mathrm{Cd})$ can accumulate in the body and be very toxic to humans and other organisms. Multiple studies have shown that cows near industrial sites and other areas polluted by these contaminants produced milk that had a higher level of these metals than cows in unpolluted areas [17, 18]. Other elements have also shown to change depending on the environment of the cow. Hermansen et al. have reported differences in elements such as $\mathrm{Mo}, \mathrm{Mn}$, and $\mathrm{Zn}$ in organic farm systems (not 
treated with antibiotics) vs. conventional farms. When it comes to different feeds, Coni et al. noticed significant differences in the element composition of raw milk from the winter and summer seasons. They hypothesized that these differences are caused by changes in the feeding systems [19]. The correlation between trace elements in milk and the elemental composition of feed were also reported in Herwig et al. [20] and SolaLarrañaga et al [21]. These differences in elemental profiles suggest that multi-elemental analysis of milk has the potential to be used to differentiate milk samples originating from farms with different feeding systems and environments and thus possibly by country if the within country variation is less than the between country variation. There have been recent studies that looked at using the elemental profile of milk for provenancing. Potocnik et al. [6] was able to classify milk samples based on geographic region within Slovenia. Sacco et al. [22] was able to classify milk as being from southern Italy or another area, foreign milk.

\section{$1.4 \quad$ Milk Production}

\subsubsection{Collection and Storage}

In the United States, the processing of milk is a very standardized process. After milking, the milk from a farm is stored in bulk cooling tanks. Each day a milk tanker will travel to farms, collect the milk, and transport it to a dairy facility. When it arrives at the dairy facility, the quality of the milk is checked. If no problems are found, it is stored in silos until it can be processed. Silos typically have a capacity of $100,000 \mathrm{~L}$ to $500,000 \mathrm{~L}$ [8]. 


\subsubsection{Standardization of Fat Content}

The fat/cream is separated from the milk using centrifugation. Depending on the final product, the cream is added back to the milk in a set amount. Even in the process of creating whole milk, the cream is removed and added back to the milk to ensure the milk has a fat content of the desired standardized amount.

\subsubsection{Heat Treatment}

Milk is heat treated to kill most of the microorganisms. However, heating milk to high temperatures also involves a risk of adverse effects on the appearance, taste and nutritional value of the milk. Today, most heat treatments on milk intended to remain a liquid consist of high temperatures for a short amount of time. This process involves heating milk to $72-75{ }^{\circ} \mathrm{C}$ and holding this temperature for $15-20$ seconds before it is cooled. Temperatures around $70{ }^{\circ} \mathrm{C}$ are high enough to kill one of the toughest bacteria, Tubercle bacillus. The heat treatment described above is called HTST (high temperature short time) pasteurization [8]. However for milk that is designed for longer storage, such as powdered milk, higher temperatures are needed. These higher temperatures not only kill pathogens, but they also inactivate enzymes and cause oxidative resistance. For milk powder, heat treatment is commonly performed at $85-95^{\circ} \mathrm{C}$ for $15-30$ seconds [23].

\subsubsection{Powdered Milk}

For powdered milks, the next steps involve evaporation of the water component, which accounts for over $80 \%$ of the milk. This process requires a lot of energy and thus is usually expensive. Care needs to be taken to not alter the proteins, particularly whey, which are sensitive to high temperatures. There are many evaporation techniques that 
differ by equipment type and energy needed. Each evaporation unit must meet three industry requirements: high evaporation capacity, low energy consumption, and ability to maintain the quality of the milk powder [24].

To reduce the energy needed, multiple single stage evaporation units can be used in a series, Figure 4. The evaporators are kept under a partial vacuum, which reduces the temperature needed. As milk at its boiling temperature enters the first evaporator, it makes contact with heated vapor that is about $5{ }^{\circ} \mathrm{C}$ hotter. This extra heat causes vaporization of some of the liquid. The slightly condensed milk and vapor are separated. The vapor is used to heat the milk as it travels through the next evaporation unit, which is at a lower pressure than the unit before it [24].

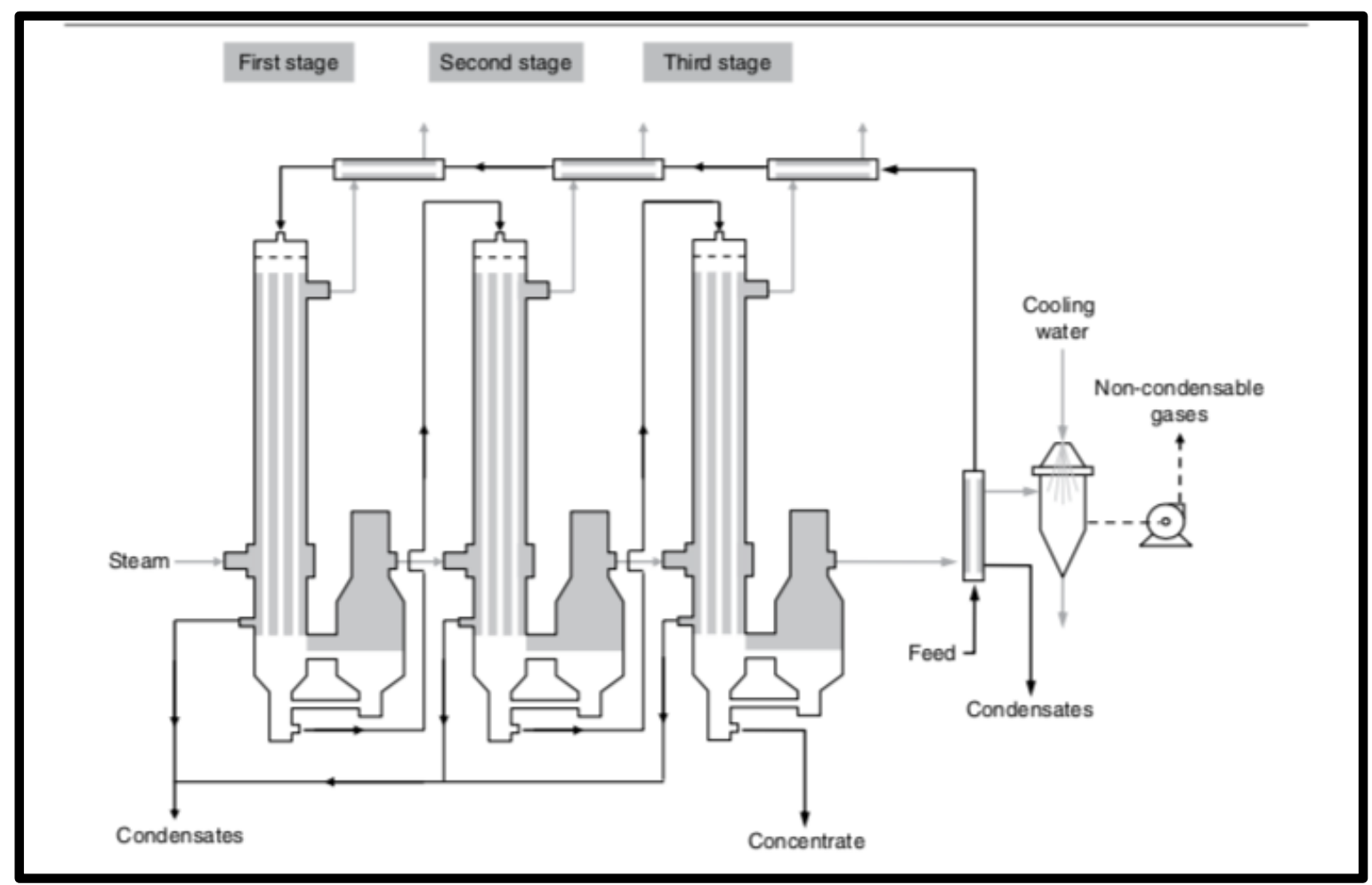

Figure 4 - The multi-stage evaporation process used to concentrate milk 
After concentrating the milk solids, the milk is homogenized to prevent creaming and separation of the fat content in the concentrated milk. The homogenization process basically reduces the size of the fat globules [23]. In cases like skim milk, the homogenization step is skipped because of the low abundance of fat molecules. The process can end here if the desired product is milk concentrate.

The next step to create milk powder involves drying the milk concentrate into a powdered form, which is usually done today by spray drying. In spray drying the milk is atomized into a hot air stream of $180{ }^{\circ} \mathrm{C}$. The milk powder is moved through this process quickly to prevent overheating [24]. 


\section{CHAPTER 2. Elemental Analysis of Milk}

As previously discussed, past publications on the elemental analysis of bovine milk and milk powder have shown that the elemental concentrations in milk statistically differ by seasons, feed, and the location of the farm. Being able to determine if an imported milk has an elemental profile that is consistant with the country listed on the label is one step towards preventing milk adulteration.

The most commonly used techniques for the elemental analyses of milk (liquid) are inductively coupled plasma-optical emission spectroscopy (ICP-OES) $[25,26]$ and inductively coupled plasma-mass spectroscopy (ICP-MS) [20, 27]. However, for both of these methods, the sample usually must undergo an acid digestion procedure and dilution prior to analysis. Other researchers have reported the use of a specialized nebulizer and direct sampling of either a diluted milk sample or a milk sample treated with EDTA [26, 28, 29].

An alternative to these liquid sample introduction approaches is laser ablation (LA). A laser is used to generate fine particles of a solid sample that can be further analyzed by elemental techniques, thus eliminating the need for strong acids and dilutions, and allows for a larger representative sample to be characterized. Methods for preparation of solid milk samples have been previously developed for both laser induced breakdown spectroscopy (LIBS) and LA-ICP-OES [30-33]. These sample preparation methods have either reported problems with matrix effects or were limited to only the major elements $\mathrm{Na}, \mathrm{Ca}, \mathrm{Mg}$, and $\mathrm{K}$. 


\subsection{Principles of Analytical Techniques}

My research focused on the development of an LA-ICP-MS method for the analysis of powdered milks, which will be compared to $k_{0}$-INAA. LA-ICP-MS is also used for the analysis of glass in the second part of this dissertation. The principles of lasers and ICP-MS can be found within the glass portion in section 6.6 . This section will focus solely on the principles of neutron activation analysis (NAA).

\subsection{1 $k_{0}$-Instrumental Neutron Activation Analysis}

Neutron Activation Analysis (NAA) is known as a primary method of measurement. Primary methods can be defined as "...a method having the highest metrological properties, whose operation can be completely described and understood, for which a complete uncertainty statement can be written down in terms of SI units [34]." By looking at this definition, it can be seen why scientists employ NAA for certification of references materials. The IAEA-153 milk standard, which is used throughout this study is not a certified reference material, but rather instead contains only recommended values. To better ensure the accuracy of the developed methods, NAA was used and the results were compared to those obtained by the LA-ICP-MS method,

In simplistic terms, neutron activation analysis is an elemental technique in which neutrons are used to irradiate a sample. The irradiation causes the formation of radioactive species, which will decay and emit excess energy in the form of gamma rays. Gamma rays have a discrete energy and are characteristic of an element, which in turn can be used to calculate the concentration of an element. Equation (1) is used here to describe the theory behind neutron activation analysis. 


$$
R=\psi \varphi \sigma N\left(1-e^{-\lambda t_{i r r}}\right) e^{-\lambda t_{c o o l}}
$$

\section{Equation (1)}

In this equation, $R$ is the amount of radioactive nuclide, $N$ is the number of target atoms $/ \mathrm{cm}^{3}$ present in the sample, $\varphi$ is the particle flux, $\psi$ is the efficiency of the detector, $\sigma$ is the reaction cross section, $t_{i r r}$ is the irradiation time, $t_{c o o l}$ is the time from the end of bombardment to the start of measurement, and $\lambda$ is the decay constant of the radionuclide [35]. Using the equation, the amount of radionuclide produced is directly related to irradiation time and particle flux from the neutron source. The analyst can control these. However, the decay constant of the radionuclide and the reaction cross-section cannot be controlled. The decay constant can limit the element menu. For example elements such as $\mathrm{Si}, \mathrm{Al}, \mathrm{Fe}$, and $\mathrm{Mg}$ produce radionuclides that have very short half-lives (2.27 minutes, 9.45 minutes, 2.58 hours, and 15 hours respectively) [36]. When a large element menu is examined, these elements could already be undetectable before the sample is even done being irradiated. Some elements have a very small cross section that reduces the probability a reaction with a neutron occurring. Therefore, these elements are not activated easily and require longer irradiation times and/or a larger neutron flux. The amount of time and neutron flux needed to activate these elements may not be economically feasible.

\subsubsection{Activation}

Neutron activation analysis is based on the generation of a radioactive species, a form of the element of interest that is unstable and thus decays to a more stable form. In neutron activation, the generation of the radioactive species is usually done by a neutron capture reaction, which is shown below, Equation (2). 


$$
{ }_{11}^{23} \mathrm{Na}+{ }_{0}^{1} n \rightarrow{ }_{11}^{24} \mathrm{Na}+\gamma
$$

Equation (2)

In neutron capture, a neutron $\left({ }_{0}^{1} n\right)$ is added to the nucleus of the element, which forms a new isotope with the same atomic number and a mass number that has been increased by 1. In this example, the binding of a neutron causes the new nucleus to have excess energy, which it releases in the form of a gamma $(\gamma)$ ray. This immediate emission of a gamma ray is called prompt gamma ray emission and is not normally measured in NAA. Rather, the gamma rays that are emitted after the decay of a radionuclide, ${ }_{11}^{24} \mathrm{Na}$, into an excited new species and its relaxation to a ground state are measured.

There are three sources of neutrons that can be employed in neutron activation analysis: reactors, radionuclides, and accelerators. These sources differ in their neutron flux and thus their detection limits. My research used a nuclear reactor to generate the neutrons, so that will be the main topic discussed. For information about radionuclides and accelerators, please refer to Skoog et al. [37]. The nuclear reactor used in this research was a TRIGA (Training, Research, Isotopes, General Atomic) Mark II reactor, which is used for research and not for energy production.

The principle component in any reactor is the core rods that contain the fuel, enriched $\mathrm{UO}_{2}$. The nuclear reactor creates neutrons through fission, a process in which a neutron collides with an isotope and causes the atom to split into two smaller nuclides and also produces neutrons. ${ }^{235} \mathrm{U}$ is a species that will undergo fission, Equation (3) [35].

$$
{ }_{92}^{235} U+n \rightarrow \text { Fission products }+\sim 2.5 n
$$

\section{Equation (3)}

The energy that the neutrons have can be classified as fast, thermal, or epithermal, Figure 5. The neutrons that are released after fission are fast neutrons. These are usually 
slowed by a moderating species, which allows them to be captured by more ${ }^{235} \mathrm{U}$ and thus continue the creation of neutrons, or they are slowed and used to activate the sample.

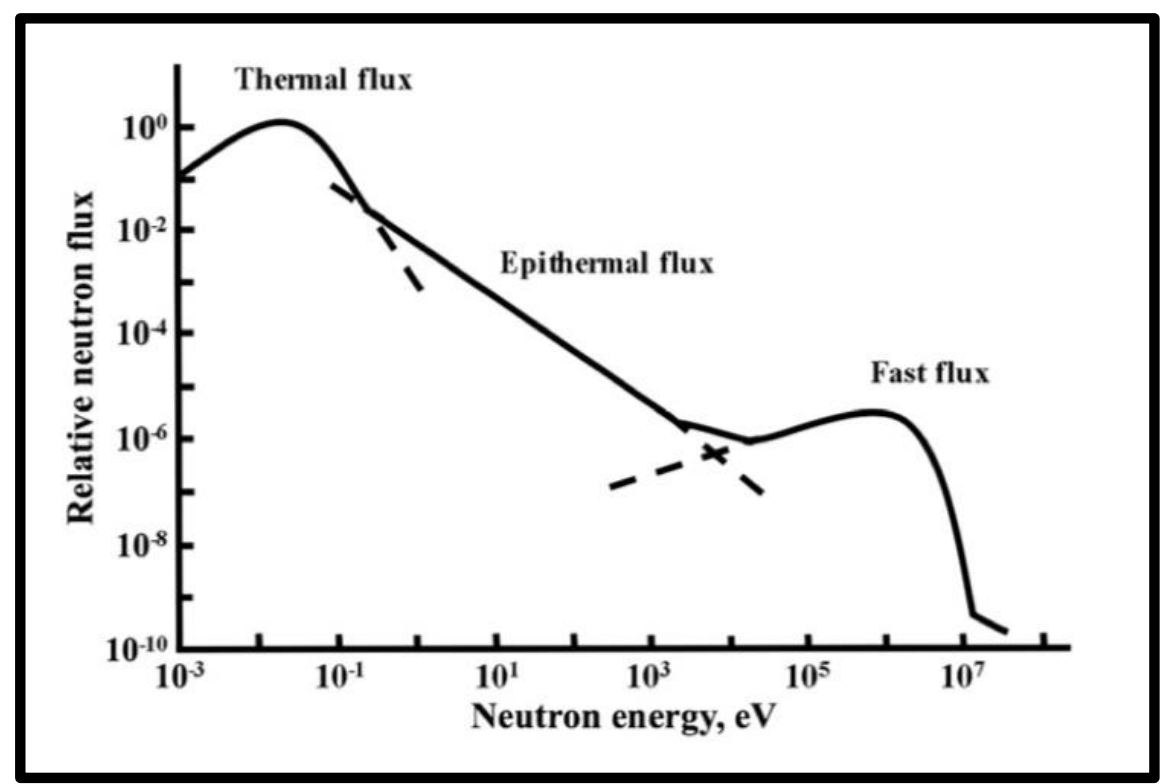

Figure 5 - Neutron energy and flux found in reactors [34]; thermal neutrons $\left(10^{-1}-10 \mathrm{eV}\right)$ are used to induce fission and activate most elements

\subsubsection{Types of Decay}

There are a variety of reactions that occur in NAA. The target element can become activated and decay into a new element. Two of the main decay methods are alpha and beta decay. Beta decay is the emission of positrons $\left({ }_{+1}^{0} \beta\right)$, beta $\operatorname{particle}\left({ }_{-1}^{0} \beta\right)$, or the capture of an electron $\left({ }_{-1}^{0} e^{-}\right)$.

$$
{ }_{Z}^{A} X \rightarrow{ }_{Z-1}^{A} X^{-}+{ }_{+1}^{0} \beta+v \rightarrow{ }_{Z-1}^{A} X+{ }_{-1}^{0} e^{-}+{ }_{+1}^{0} \beta+v \quad \text { Equation (4) }
$$

$$
{ }_{Z}^{A} X \rightarrow{ }_{Z+1}^{A} X+{ }_{-1}^{0} \beta+\bar{v}
$$




$$
{ }_{Z}^{A} X \stackrel{E C}{\rightarrow} \underset{Z-1}{A} X+v
$$

Equation (6)

Equation (4), Equation (5), and Equation (6) show examples of these processes where X is an atom with $\mathrm{A}$ atomic number and $\mathrm{Z}$ mass number [35].

$$
{ }_{Z}^{A} X \rightarrow{ }_{Z-2}^{A-4} X+{ }_{2}^{4} \mathrm{He}
$$

Equation (7)

Alpha decay is the emission of a helium nucleus, Equation (7). Alpha and beta decay can leave the product's nucleus in an excited state. The excess energy can either be emitted as a gamma ray or lost by a process called internal conversion. The gamma rays are of importance to NAA and thus will be discussed further.

Gamma rays are high-energy radiation that have been emitted from an excited nucleus. They have the same electromagnetic nature as X-rays and like X-rays produce line spectra in which identification of an element can be determined. In NAA, the gamma rays that are detected are usually from delayed gamma emissions, an emission that occurs after a radionuclide decayed. Since the gammas are released after a radionuclide decayed, the gamma ray being examined to determine the concentration of a target element can be a gamma ray emitted from the relaxation of a different element. This can become confusing and complicated, but fortunately computer software and literature sources exist that analyze decays schemes and calculate the probability of each event occurring. An example of a decay scheme for metastable ${ }^{60} \mathrm{Co}$ can be seen in Figure 6. First the metastable species undergoes an isomeric transition (IT) to ${ }^{60} \mathrm{Co}$ by emission of a gamma ray. Next the species undergoes beta decay by emission of a beta particle to an excited ${ }^{60} \mathrm{Ni}^{*}$. The nickel isotope will undergo emission of two more gamma rays till it is in a ground state [34]. 


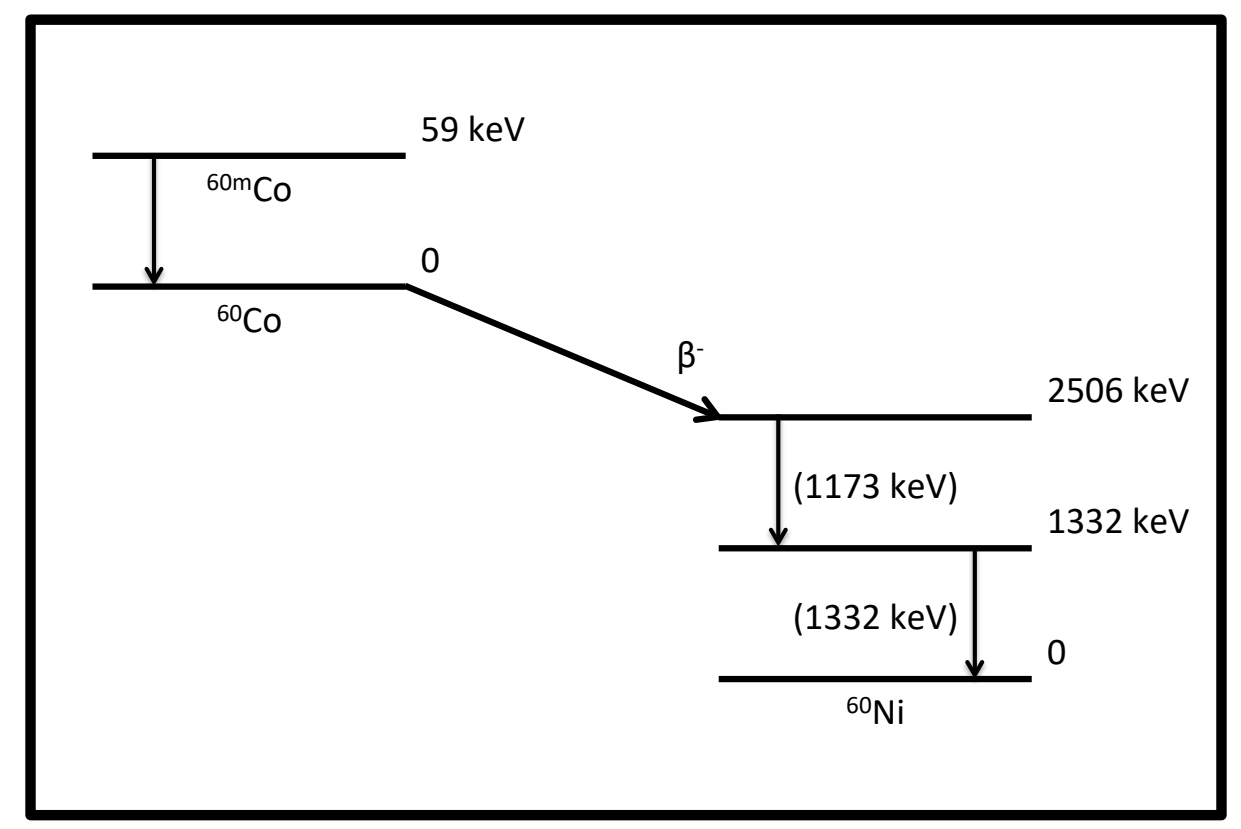

Figure 6 - Decay scheme for ${ }^{60 \mathrm{~m}} \mathrm{Co}$ into ${ }^{60} \mathrm{Ni}$; during the decay two gamma rays are emitted with energy of $1173 \mathrm{keV}$ and $1332 \mathrm{keV}$

\subsubsection{Detection}

The interaction of gamma radiation with matter results in ionization and generation of a current that can be detected and recorded. Detectors used can be gas filled (Geiger tubes, proportional counters, and ionization chambers), scintillation counters, and semiconductors ( $\mathrm{Si}(\mathrm{Li}), \mathrm{Ge}(\mathrm{Li})$, and $\mathrm{HPGe})$. In NAA, semiconductors are most commonly used because of their high-energy resolution, thus these will be discussed in detail. For information about the other detectors see Skoog et al. or Cherry et al [37, 38].

In semiconductor detectors, ionizing radiation interacts with solid matter (crystal) to produce electron hole pairs. The electrons have a negative charge and the holes carry a positive charge. By applying an external electric field, these charge carriers can be separated to create an electric field within the detector. This electric field creates a pulse that has a height equivalent to the energy of the radiation. 
As photons, gamma rays, enter into the detector three events can occur. The gamma ray can interact with an atom, lose all of its energy, and cause the ejection of an electron (photoelectric effect), which creates a hole in the atom's electron shell. The atom then moves an electron from a higher energy shell down to fill the hole, which in turn emits an X-ray. The energy of the photoelectron and emitted x-ray is equal to the energy of the gamma ray. This interaction produces a full energy peak, a peak with energy equal to the gamma ray. Photoelectric effect is the best-case scenario. Another event that can occur is that the photon interacts with the outer shell electrons and only loses some of its energy (Compton scattering). The photon is scattered and the event can continue until the photon has lost all of its energy inside the detector. If this happens, a full energy peak is created. However, there is a possibility that the scattering will cause the photon to escape out of the detector, which results in less energy being detected. This event produces the Compton continuum of a gamma spectrum. The third interaction occurs with gamma rays that have energy equal to or greater than $1022 \mathrm{keV}$. These high-energy gamma rays can interact with the electric field of a nucleus. This causes the conversion of a proton into an electron-positron pair (pair-production). The energy of the gamma ray in excess of $1022 \mathrm{keV}$ is carried as kinetic energy by each particle. When the positron has lost all of its kinetic energy, it will meet an electron and produce two $511 \mathrm{keV}$ annihilation photons. These photons can deposit their energy in the absorber, or one or both can escape the detector. These phenomena result in the formation of a full energy peak, a peak equal to the full energy minus $511 \mathrm{keV}$, or a full energy peak minus $1022 \mathrm{keV}$, respectively [34]. 


\subsubsection{Determining Concentration with the $k_{\mathrm{o}}$ Method}

The method described above using a semiconductor detector is often referred to as instrumental (INAA). INAA allows the simultaneous and quantitative analysis of multiple elements. The use of detector with less resolution can require some chemical purification to eliminate radionuclides that interfere with the signal from your target element. This is called radiochemical NAA (RNAA).

In INAA analysis, the concentration of an unknown sample can be accurately determined by irradiating and analyzing a matrix-matched reference standard with the unknown. The activity of the element in the unknown can be determined by comparing it to the activity of the element in the reference standard, Equation (8) [35]

$$
\frac{\text { weight of element in unknown }}{\text { weight of element in standard }}=\frac{\text { activity of element in unknown }}{\text { activity of element in standard }}
$$

Equation (8)

However, sometimes a matrix-matched standard is unavailable. This is where the $k_{0}$ approach comes in. It is possible to calculate the concentration of an element by using the cross section of that element, neutron flux, irradiation time, and half-life.

In $k_{0}$ INAA, a comparator is used instead of a matrix-matched standard. In this research the comparator was a gold alloy. The benefits of using gold are that it has only one stable isotope, the location of the gamma peak is in a well-calibrated area, and it has a short decay time. Using Equation (9), the $k_{0}$ value for an analyte and comparator (Au) can be calculated.

$$
k_{0, A u}(a)=\frac{M_{A u} \times \theta_{a} \times \sigma_{0, a} \times \gamma_{a}}{M_{a} \times \theta_{A u} \times \sigma_{0, A u} \times \gamma_{A u}}
$$

$M$ is the mass. $\theta$ is the isotopic abundance. $\sigma$ is the cross section 
The $k_{0}$ for a comparator and analyte are independent of the neutron source and detector used and only require literature values. The calculated $k_{0}$ value can then be used in Equation (10) to calculate the concentration of an analyte in the unknown sample.

$$
\varrho_{\mathrm{a}}=\frac{\left(N_{\mathrm{p}} / W t_{\mathrm{m}} S D C\right)_{\mathrm{a}}}{\left(N_{\mathrm{p}} / w t_{\mathrm{m}} S D C\right)_{\mathrm{Au}}} \times \frac{1}{k_{0, \mathrm{Au}}(\mathrm{a})} \times \frac{f+Q_{0, \mathrm{Au}}(\alpha)}{f+Q_{0, \mathrm{a}}(\alpha)} \times \frac{\varepsilon_{\mathrm{p}, \mathrm{Au}}}{\varepsilon_{\mathrm{p}, \mathrm{a}}} \quad \text { Equation (10) }
$$

$Q_{a}$ is the concentration of the analyte. $\mathrm{N}$ is the net number of counts in the full energy peak. $\mathrm{W}$ is the weight. $\mathrm{t}_{\mathrm{m}}$ is the measuring time. SDC is based off of the half-life, irradiation time, and decay time. $\mathrm{f}$ is the measure of thermal to epithermal neutron flux. $\mathrm{Q}$ is the resonance integral to $2200 \mathrm{~m} / \mathrm{s} . \alpha$ is a measure for the epithermal flux distribution. $\varepsilon$ is a measure of the detector efficiency for the full energy peak [34].

As can be seen by Equation (10), INAA requires background information on the nuclear reactor and detector used.

2.2 Materials and Methods for Analysis of Milk

Some of the data in this chapter has been published in T. Hoffman et al. Development of a Method for the Elemental Analysis of Milk Powders Using Laser Ablation-Inductively Coupled Plasma-Mass Spectrometry (LA-ICP-MS) and its Potential Use in Geographic Sourcing. Talanta 2018 [39].

\subsubsection{Milk Powder Sample Set}

Two reference standards and a casein powder were used for quality control and calibration. The sample set used for the present study consisted of commercial milk powders packaged and sold in grocery stores and two types of "authentic" milk powders. "Authentic" is defined here as a sample where the country of origin is known and is 
documented. These are milk powders collected directly from a drying facility and milk that was collected directly from a specific farm and dried in a laboratory. The collection of physical milk powders was a difficult task because of a variety of reasons. First, within the USA milk powder adulteration is not discussed much. This could be because the public is not knowledgeable of it occurring or because it does not occur. Many of the dairy industries and dairy programs who were contacted were not eager to help supply samples. Second, obtaining samples from other countries was challenging because of exporting and importing laws. Some samples were stopped at the USA border and returned to the sender. Third, the size of the samples collected by other countries was not enough to share. For these reasons, the data from "authentic" samples and not physical samples were collected and evaluated.

\subsubsection{Reference Standards}

The following reference materials were used: IAEA-153 (Trace Elements in Milk Powder) and IAEA-155 (Trace Elements in Whey Powder). These materials contained recommended and informational concentrations for the elements of interest.

\subsubsection{Casein}

The IAEA sent out a casein sample to all participants. There are no reported elemental values for the sample. The values used in this study were obtained from another lab that digested the casein and analyzed it with ICP-MS. These values are presented in Table 3 and represent the means from three separate digests. 
Table 3 - Concentration of elements of interest in casein determined by solution ICP-MS

\begin{tabular}{|ccc|}
\hline Element & \multicolumn{2}{c|}{ ug / g dry weight } \\
& Mean & Standard Deviation \\
\hline${ }^{23} \mathbf{N a}$ & 7.325 & 0.002 \\
${ }^{24} \mathbf{M g}$ & 61 & 3 \\
${ }^{43} \mathbf{C a}$ & 1150.5 & 219.6 \\
${ }^{44} \mathbf{C a}$ & 1211.3 & 228.5 \\
${ }^{66} \mathbf{Z n}$ & 50.7 & 5.4 \\
${ }^{85} \mathbf{R b}$ & 0.020 & 0.001 \\
${ }^{88} \mathbf{S r}$ & 1.23 & 0.12 \\
\hline
\end{tabular}

\subsubsection{Commercial Milk Powder}

Commercial is used to describe samples purchased from a store where the exact origin is unknown. Commercial milk powder samples were collected from local grocery stores. These samples included whole milk, nonfat, and nonfat instant milk powders.

\subsubsection{Authentic Milk}

Authentic is used to describe a milk sample where the origin such as country, region, or farm of production is known and documented. Authentic United States milk powder samples were shipped directly from a milk shed drying facility in Tulare, California. Each sample contained approximately $500 \mathrm{~g}$ and was marked as either skim or nonfat dairy milk. Authentic milk samples from other countries were either collected from farms within that country by the participating laboratory and dried in the laboratory using various techniques or collected directly from a drying facility. The samples collected at the farm level were approximately $2 \mathrm{~g}$ each. 


\subsubsection{Authentic Data}

Each lab collected authentic milk samples, analyzed them using their own method, and submitted the data. Countries represented include: Argentina, Russia, Singapore, Slovenia, and the United States. For some countries, the data submitted represents a small area and not the entire country. For example, the United States data represents 3 samples from a milk shed. The Slovenia data represents 2 drying facilities, 2 samples from each. The data from Singapore represents 2 farms, 5 samples from each. The data from Argentina are from 1-3 farms within each of 4 distinct regions. The data spread from Russia is for 41 samples collected from farms located throughout the southern half of the entire country $(\sim 9000 \mathrm{~km})$.

\section{$2.3 \mathrm{k} 0$-INAA}

\subsubsection{Preparation of Milk Samples for $\mathrm{k}_{0}$-INAA}

For $\mathrm{k}_{0}$-INAA an aliquot $(0.06$ to $0.24 \mathrm{~g})$ of milk powder sample was measured into a pure polyethylene ampoule (SPRONK system, Lexmond, Netherlands) so that the ampoule was full and sealed. For determination of intermediate and long-lived radionuclides, the milk powder and standard Al-0.1\% Au (IRMM-530R) discs were stacked together and fixed in the polyethylene vial in sandwich form, Figure 7. Sandwich form makes it easier to account for variations caused differences between the distances of the Al-Au standards and milk samples from the neutron source. Using the sandwich form, both have the same $\mathrm{x}$-distance so only variations in the $\mathrm{y}$-distance need to be corrected. This is done by taking the average of the Al-Au standards located above and below each milk sample. 


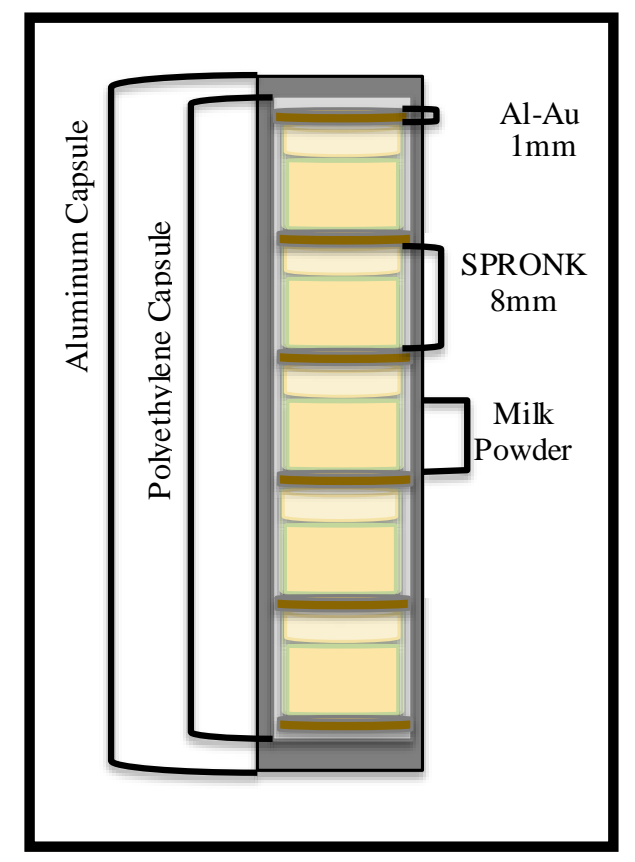

Figure 7 - Sample preparation for $\boldsymbol{k}_{0}$-INAA analysis

\subsection{2 $\mathrm{k}_{0}$-INAA Instrument Parameters}

The samples and standards were irradiated for $12-20$ hours in the carousel facility of the TRIGA reactor with a thermal neutron flux of $1.1 \times 10^{12} \mathrm{~cm}^{-2} \mathrm{~s}^{-1}$. After irradiation, the milk powder was measured after 4,7 and 21 days cooling time on absolutely calibrated HPGe detectors with 40 and $45 \%$ relative efficiency. The Al-Au discs were analyzed after 4 days for 240 live seconds. The milk powder was analyzed after 4 days for 3600 live seconds and again after 10 days for 60000 live seconds, and again after 21 days for 52900 live seconds. The measurement distance of the samples from the detectors was set to achieve a dead time of below 10\%. Figure 8 and Figure 9 show examples of the gamma spectra for the Au Comparator and the IAEA 153 standard. 


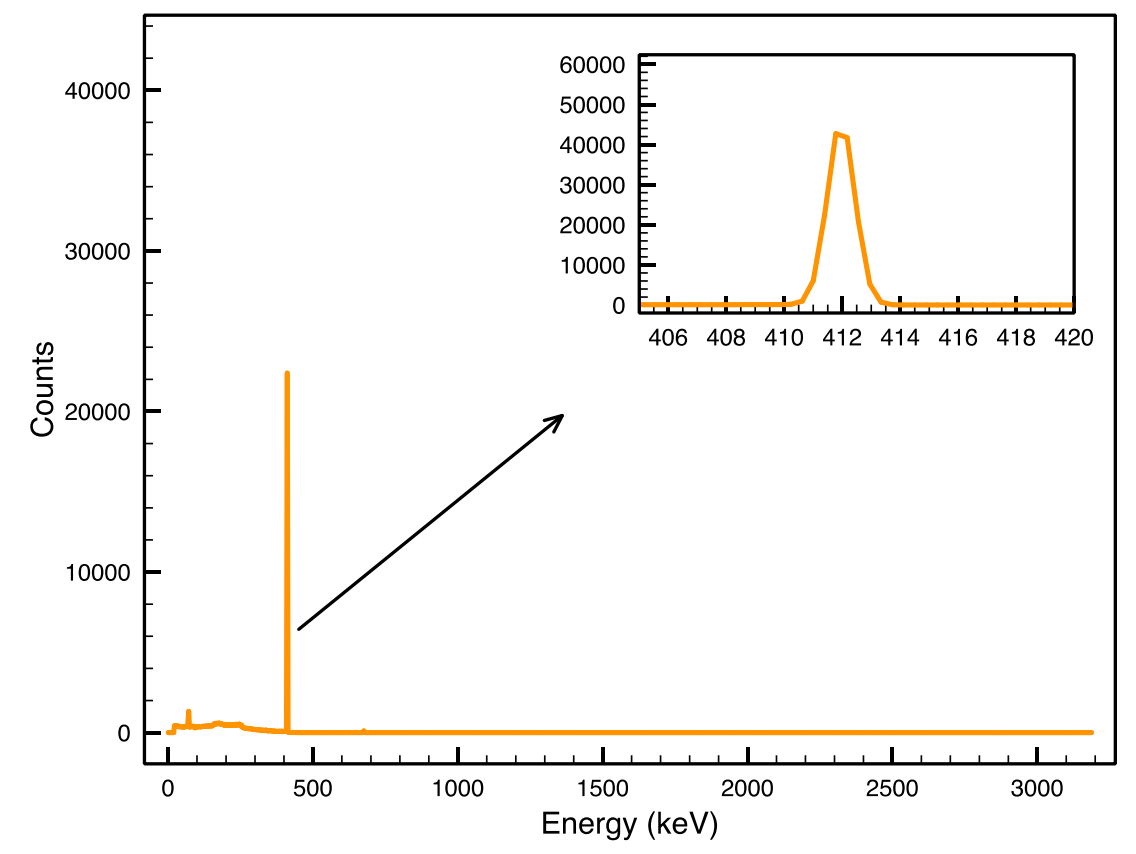

Figure 8 - Gamma spectrum of the gold comparator: 240 live seconds

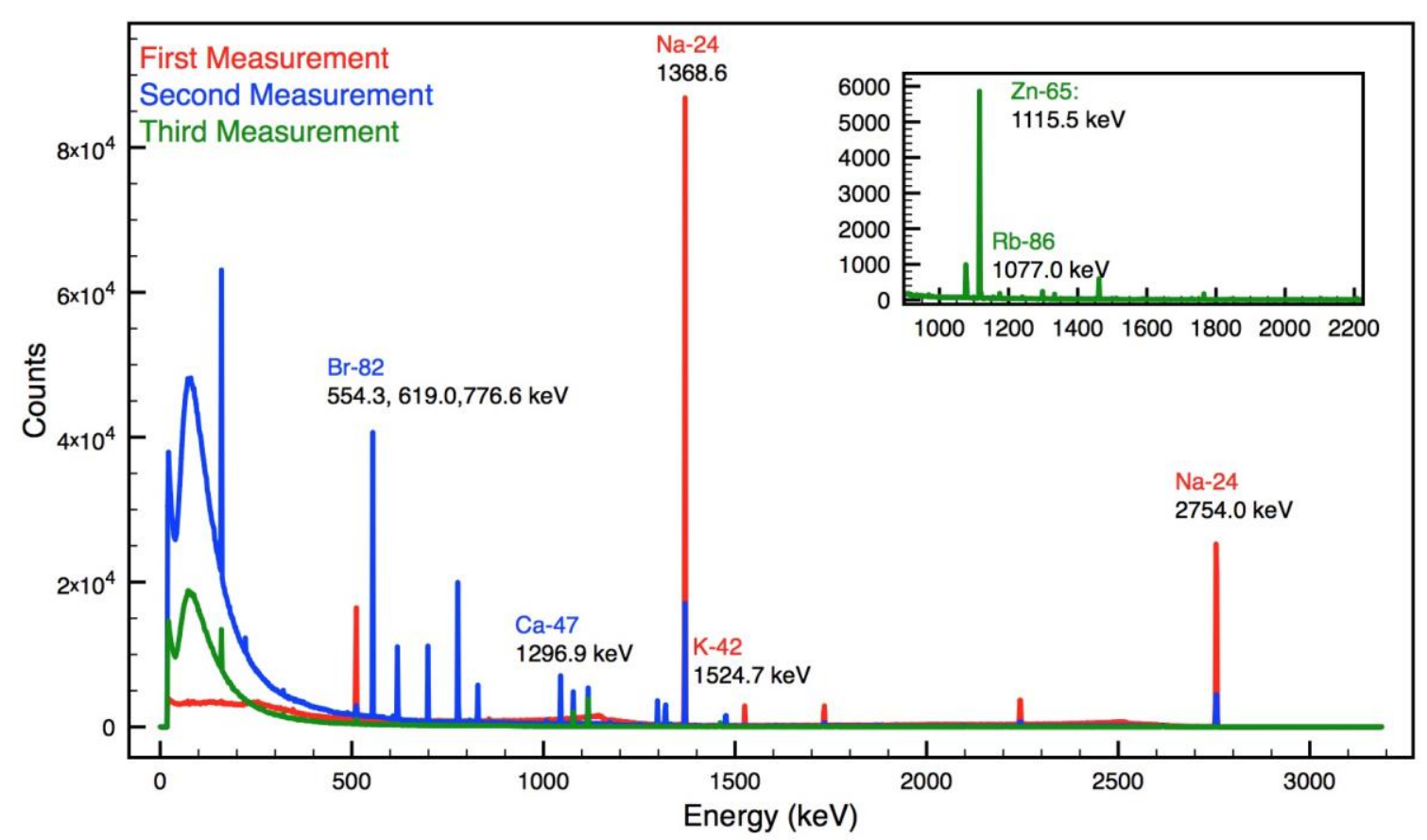

Figure 9 - Gamma spectrum for IAEA-153: The first measurement is in red and was accumulated for 3600 live seconds. The second measurement is shown in blue and was accumulated for 60000 live seconds. The third measurement is in green and was accumulated for $\mathbf{5 2 9 0 0}$ live seconds. 


\subsection{3 ko-INAA Data Analysis}

For peak area evaluation, the HyperLab 2002 program (Hyperlabs Software, Budapest, Hungary) was used. This software fixes resolution, defines peaks to use, and defines the background. HyperLab was also used to determine $f$, which is the flux ratio of thermal to epithermal neutrons, and $\alpha$, the deviation of the epithermal flux from the ideal distribution.

\subsection{4 k0-INAA Statistical Analysis}

For elemental concentrations and effective solid angle calculations the software package Kayzero for Windows (DSM Research, Geleen, Netherlands) was applied. The software takes into account the sample mass and density, the distance the sample was from the detector, and the time of irradiation to calculate concentration. Since the samples were sandwiched between two Al-Au discs the average $\mathrm{F}_{\mathrm{c}}$ (flux ratio for the comparator) value of the two discs was used.

\subsection{LA-ICP-MS Analysis}

\subsubsection{Preparation of Milk Samples for LA-ICP-MS}

Three different preparation methods were created that had reasonable \%bias $(<10 \%)$ and precision $(<10 \%)$ for most evaluated elements. The first method will be referred to as pelleting method \#1. This method involves the creation of a standard addition curve by the addition of solution spikes to a sample of milk powder followed by milling and pelleting the powder. The second method will be referred to as pelleting method \#2. This method involves the creation of an external calibration curve by mixing various amounts of IAEA-155 and the casein powder followed by milling and pelleting. 
The third method will be referred to as the dried spot method. This sample preparation was developed by Aramendia et al. and previously used by Nischkauer et al. for the analysis of phosphorus in milk powder [40, 41].

\subsubsection{Element Reagents}

For the creation of calibration curves and addition of internal standards, single element ICP (CPI International, Santa Rosa, CA) and ICP-MS (Ricca Chemical Company, Pocomoke City, MD) standard solutions of $\mathrm{Zn}, \mathrm{Rb}, \mathrm{Sr}, \mathrm{Y}$, and In at 1000 $\mathrm{ug} / \mathrm{mL}$ and $\mathrm{Na}, \mathrm{Mg}, \mathrm{Ca}, \mathrm{Sc}$ at $10,000 \mathrm{ug} / \mathrm{mL}$ were used.

\subsubsection{Sample Preparation for Pelleting Method \#1}

Four representative samples of approximately $0.5 \mathrm{~g}$ of milk powder were accurately weighed to a precision of $0.1 \mathrm{mg}$ into $17 \times 100 \mathrm{~mm}$ polypropylene tubes. When sample mass was scarce, only $0.15 \mathrm{~g}$ of powder was used. Scandium and indium single element ICP-MS standard solutions were added ("spiked") as internal standards to each sample to a final concentration of 500 and 50 ppm, respectively.

Since the ICP element standards used are in nitric acid, partial digestion of the milk powders could occur. Calibration solutions were prepared that allowed the same amount of solution ( $160 \mu \mathrm{L}$ for $0.5 \mathrm{~g})$ to be added to each sample. Samples were mixed thoroughly with a vortex touch mixer (Fischer Scientific, Pittsburgh, PA) and dried in air at $80^{\circ} \mathrm{C}$ in a PTFE-coated graphite HotBlock (Environmental Express, Charleston, SC) for up to $12 \mathrm{hrs}$.

To prepare the pellets, the dried milk powder samples were homogenized and pulverized to a fine powder using a high speed ball mixer mill (Glenmills, Clifton, NJ, 
USA) with a tungsten carbide ball and cups at $25 \mathrm{~Hz}$ for $15 \mathrm{~min}$. For samples with a mass of $0.5 \mathrm{~g}$, the samples were pressed into pellets of $13 \mathrm{~mm}$ in diameter in a $13 \mathrm{~mm}$ stainless steel die at 4 tons for 2 minutes (Carver Benchtop Pellet Press, IN, USA). For samples with a mass of $0.15 \mathrm{~g}$, the samples were pressed into pellets of $6 \mathrm{~mm}$ in diameter in a 6 $\mathrm{mm}$ stainless steel die at 2 tons for $2 \mathrm{~min}$.

\subsubsection{Sample Preparation for Pelleting Method \#2}

An external calibration curve was created by mixing the IAEA-155 whey powder with the casein for a total mass of $0.5 \mathrm{~g}$. The mixtures used were $10 \%, 25 \%, 50 \%, 75 \%$, and 90\% IAEA-155 whey powder. Yttrium single element ICP-MS standard solution was added ("spiked") as an internal standard to each sample to a final concentration of 1000 ppm. Samples were mixed thoroughly with a vortex touch mixer (Fischer Scientific, Pittsburgh, PA) and dried in air at $80^{\circ} \mathrm{C}$ in a PTFE-coated graphite HotBlock (Environmental Express, Charleston, SC) for up to $12 \mathrm{hrs.}$

To prepare the pellets, the samples were homogenized and pulverized to a fine powder using a high speed ball mixer mill (Glenmills, Clifton, NJ, USA) with a tungsten carbide ball and cups at $25 \mathrm{~Hz}$ for 10 minutes and pressed into pellets of $13 \mathrm{~mm}$ in diameter in a $13 \mathrm{~mm}$ stainless steel die at 4 tons for 2 minutes under vacuum (Carver Benchtop Pellet Press, IN, USA).

\subsubsection{Dried Spot Method}

Circular discs of $6 \mathrm{~mm}$ were cut from Whatman 542 filter paper using a holepunch. These circular discs were attached to glass microscope cover slides using double sided tape. Samples of approximately $0.1 \mathrm{~g}$ of milk powder was weighed out and placed 
in polypropylene test tubes with snap caps (Fischer Scientific, Pittsburgh PA), and $2 \mathrm{~mL}$ of high purity water (resistivity of $18.2 \mathrm{M} \Omega \mathrm{cm}$ ) was added to each sample.

An external calibration curve was created by adding ICP-MS element solutions for the elements of interest ( $\mathrm{Na}, \mathrm{Mg}, \mathrm{Ca}, \mathrm{Zn}, \mathrm{Rb}$, and $\mathrm{Sr}$ ) to high purity water (resistivity of $18.2 \mathrm{M} \Omega \mathrm{cm}$ ). A micropipette was used to deposit $30 \mu \mathrm{L}$ of each solution onto the center of a Whatman disc. The samples were left to air-dry overnight, Error! Reference source not found.

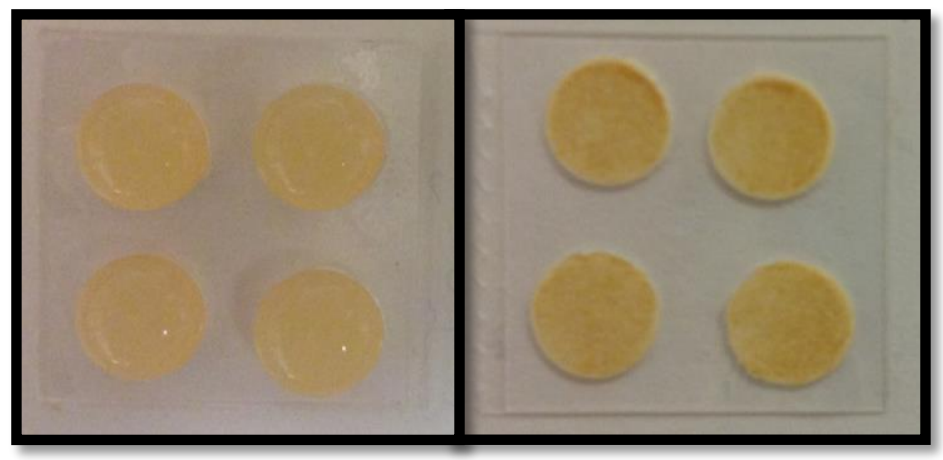

Figure 10 - Dried spot method before and after sitting overnight

\subsubsection{LA-ICP-MS Instrumental Parameters}

Elemental analysis by LA-ICP-MS was performed using a quadrupole ELAN DRC II 6100 (Perkin Elmer LAS, Shelton, CT). Argon at 16 L/minutes was used as the plasma gas with an argon auxiliary gas at $1 \mathrm{~L} /$ minutes and an RF power of $1500 \mathrm{~W}$. A 213 nm laser ablation system (New Wave Research Inc., Fremont, CA) was connected to the ICP through Tygon tubing (approximately 1.4 m, Fisher Scientific, Pittsburg, PA, USA), to a "Y" connector to merge with the argon nebulizer gas flow of $0.9 \mathrm{~L} / \mathrm{min}$, and finally to the ICP torch. Helium at $0.9 \mathrm{~L} /$ minutes was used as the ablation and carrier gas. Daily performance of the laser and ICP was tested by analyzing 7 replicate measurements of 
NIST SRM 612 (Trace Elements in Glass Matrix) (NIST, Gaithersburg, MD). The counts for a low, medium, and high molecular mass element were monitored to ensure that the instruments were consistent with normal operation. The percent of doubly charged ions and oxides were checked and had to be less than $3 \%$ before sample analysis could occur.

Isotopes that were mostly clear of interferences, easily measured, and performed well during method optimization were selected. The isotopes measured included ${ }^{23} \mathrm{Na}$, ${ }^{24} \mathrm{Mg},{ }^{39} \mathrm{~K},{ }^{44} \mathrm{Ca},{ }^{45} \mathrm{Sc},{ }^{66} \mathrm{Zn},{ }^{85} \mathrm{Rb},{ }^{88} \mathrm{Sr}$, and ${ }^{115} \mathrm{In}$. The acquisition parameters for the pellets were as follows: transient signal output with 20 s gas blank, 60 s laser ablation, and $35 \mathrm{~s}$ of post-ablation blank, peak-hopping mode with a $20 \mathrm{~s}$ dwell for each isotope in the element menu, and 5 replicates per pellet. Acquisition parameters for the dried spots were: one replicate measurement per dried spot, transient signal output with $20 \mathrm{~s}$ gas blank and $120 \mathrm{~s}$ laser ablation, followed by a $25 \mathrm{~s}$ post-ablation blank, peak-hopping mode with a $20 \mathrm{~s}$ dwell for each isotope in the element menu, and 3 sweeps per reading. Table 4 shows the optimized laser parameters for each method.

For the pellets, the laser parameters were optimized using the IAEA-153 pellet. The parameters that produced the best signal while being reproducible were chosen. The optimization experiments and results will be covered in the next chapter.

For dried spots, the laser parameters were optimized using the IAEA-153 dried spot. One of the aims during optimization was to minimize the contribution of the paper signal. The laser energy, frequency, and scan rate were all chosen based on which gave the highest reproducible signal for the milk powder while minimizing paper contribution. The optimization experiments and results will be covered in the next chapter. 
Table 4 - Laser parameters for the pelleting and dried spot sample preparations

\begin{tabular}{|lccc|}
\hline Parameter & Pelleting Method \#1 & Pelleting Method \#2 & Dried Spot \\
\hline Laser & Nd:YAG 213 nm & Nd:YAG 213 nm & Nd:YAG 213 nm \\
Mode & Spot & Spot & $6 \mathrm{~mm}$ Radial Line \\
Spot size & $190 \mu \mathrm{m}$ & $190 \mu \mathrm{m}$ & $190 \mu \mathrm{m}$ \\
Frequency & $10 \mathrm{~Hz}$ & $10 \mathrm{~Hz}$ & $10 \mathrm{~Hz}$ \\
Scan rate & - & - & $50 \mu \mathrm{m} / \mathrm{s}$ \\
\# shots & 600 & 500 & 1200 \\
Energy & $100 \%(\sim 0.65 \mathrm{~mJ})$ & $100 \%(\sim 0.65 \mathrm{~mJ})$ & $100 \%(\sim 0.65 \mathrm{~mJ})$ \\
\hline
\end{tabular}

\subsubsection{LA-ICP-MS Data Analysis}

For standard addition curves and external calibration curves, data reduction and statistical analysis were performed using Excel 2011(v14.6.2, Microsoft Corp.) and Plot software (v.0.997). For single point calibration, GLITTER (v4.4 GEMOC, Macquarie University, Sydney Australia) was used.

Figure 11 and Figure 12 depict the LA-ICP-MS transient signals for pellets and dried spots, respectively. The transient signal is a graph of counts per second (CPS) vs. time. The signal can be broken up into three regions. The first region is the gas blank. The gas blank is the first $\sim 20 \mathrm{~s}$ of analysis prior to the firing of the laser. The second region occurs when the laser is interacting with the sample. Once the laser starts firing, there is an increase in the counts. The length of time that the laser is interacting with the sample is determined by the sample type, pellet or dried spot. The third region occurs after the laser is done firing. During this time, the counts will drastically decrease. The third region is used to clean out the tubing and ablation chamber before the next replicate. 


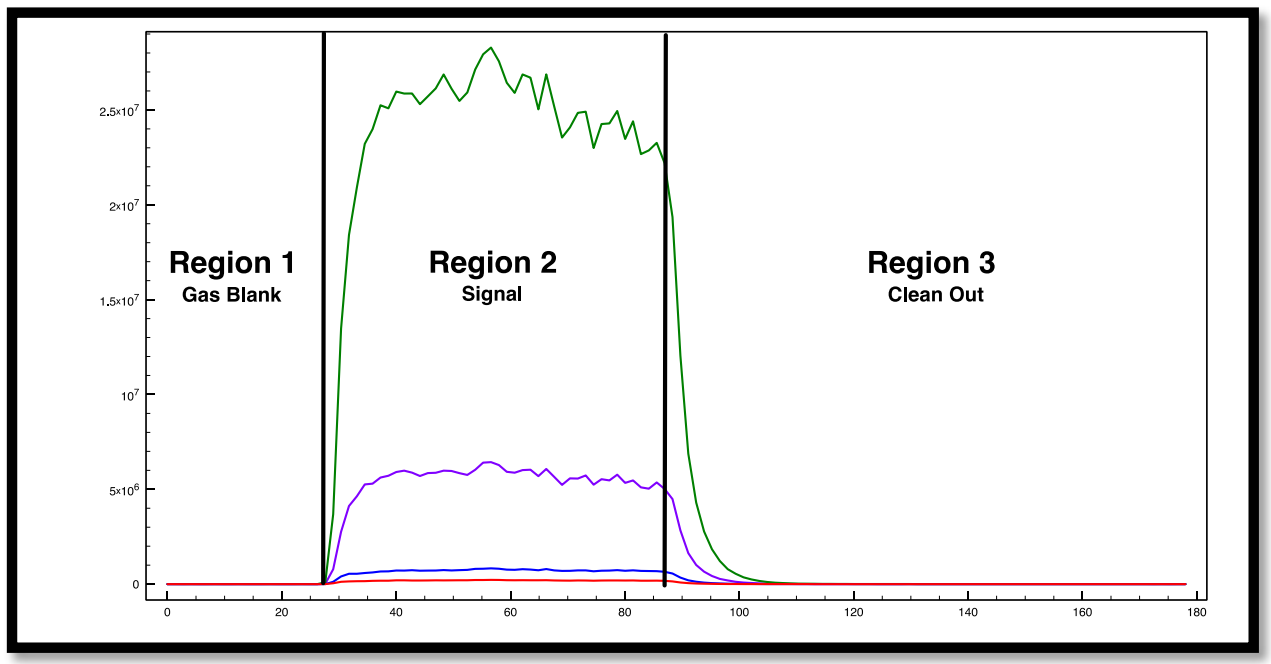

Figure 11 - The different regions of the transient signal (counts vs time) for spot analysis on pellets

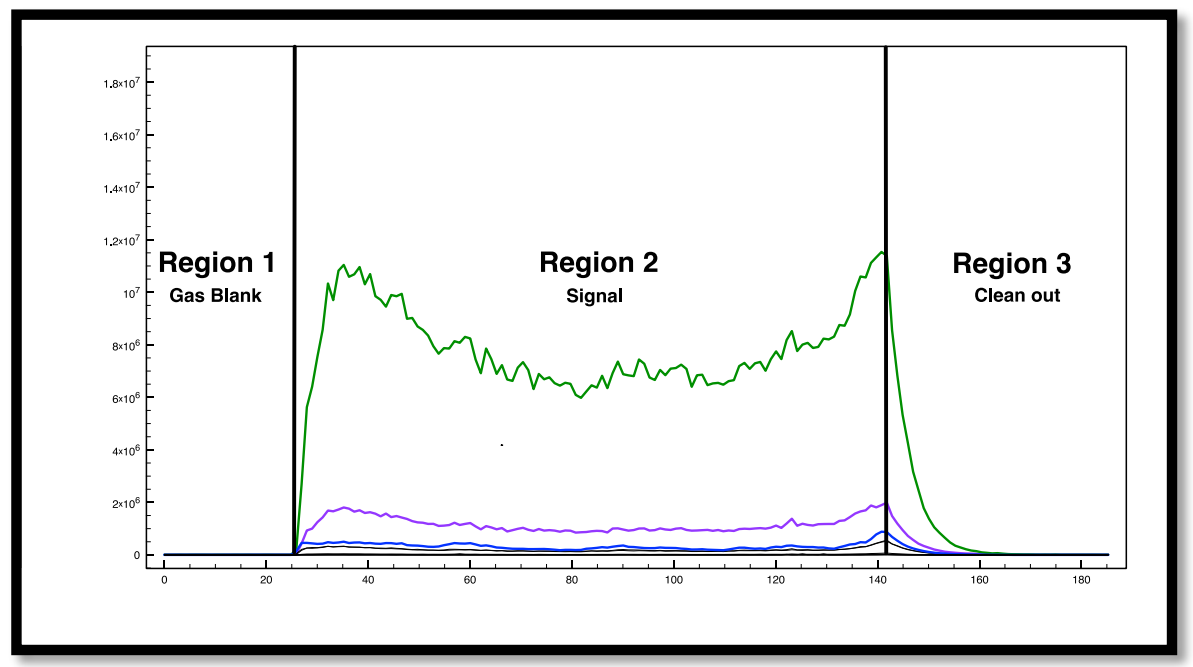

Figure 12 - The different regions for the transient signal (counts vs time) for radial line scan of the dried spots; the counts for the elements are not consistent over the entire spot. All elements of interest were found to be more concentrated on the edges of the dried spots.

A software program, Plot, was used to integrate the transient signal for standard addition curves and external calibration. For pellets, the area under the first $20 \mathrm{~s}$ of the gas blank signal was calculated. Next, ignoring the first $10 \mathrm{~s}$ of laser-sample interaction, $50 \mathrm{~s}$ of the laser-sample signal was integrated. For dried spots, the area under the first 20 
$\mathrm{s}$ of the gas blank signal was calculated. The entire laser-sample interaction, $35 \mathrm{~s}-140 \mathrm{~s}$, was integrated. Using Excel, the areas for the gas blank and signal were converted to CPS by diving each area by time. The gas blank CPS were subtracted from the signal CPS for each isotope. This blank-subtracted signal was then normalized to an internal standard.

For single point calibration, GLITTER was used. The software integrated the sample signal, subtracted the gas blank signal, and normalized the data to an internal standard. The GLITTER software also corrected for instrumental drift and calculated the concentration of each isotope in each measurement using IAEA-153 as a calibrator. Minimum detection limits (MDL) for each element were calculated by GLITTER at the $99 \%$ confidence level for each replicate measurement. Excel was used to average the replicate measurements and calculate standard deviations.

\subsubsection{LA-ICP-MS Statistical Analysis}

Two parameters that can be used to evaluate a method are accuracy (\%bias) and precision (\%Relative Standard Deviation (RSD)). The calculations for both are explained below. To test the accuracy and precision of each sample preparation method, IAEA-153 was analyzed. The \%bias was calculated for each element using:

$$
\% \text { Bias }=100 \% \times \frac{\text { Experimental Conc. }(\mathrm{ppm})-\text { Reported Conc. }(\mathrm{ppm})}{\text { Reported Conc. }(\mathrm{ppm})}
$$

Equation (11)

For the milk analysis, a \%bias less than or equal to $10 \%$ was the desired value. Another figure of merit used to evaluate the data was $\% \mathrm{RSD}$. The method precision was estimated as the uncertainty in the experimental concentration calculated from a standard addition curve, external calibration curve, or single point calibration. 
The uncertainty of the extrapolated concentration, $\mathrm{S}_{\mathrm{X}_{\mathrm{E}}}$, of a standard addition curve was calculated by using:

$$
S_{X_{E}}=\frac{S_{y / x}}{b} \sqrt{\frac{1}{n}+\frac{\bar{y}^{2}}{b^{2} \sum_{i}\left(X_{i}-\bar{x}\right)^{2}}}
$$

Where $\mathrm{b}$ is the slope of the regression line, $\mathrm{n}$ is the number of points in the regression line. $\mathrm{S}_{\mathrm{y} / \mathrm{x}}$ estimates the random errors in the $\mathrm{y}$-direction

$$
\mathrm{S}_{\mathrm{y} / \mathrm{x}}=\sqrt{\frac{\sum_{\mathrm{i}}\left(\mathrm{y}_{\mathrm{i}}-\hat{\mathrm{y}}_{\mathrm{i}}\right)^{2}}{\mathrm{n}-2}}
$$

where $\hat{y}$ are the fitted $y$-values corresponding to each $x$-value and calculated from the regression equation: $\mathrm{y}=\mathrm{bx}+\mathrm{a}$.

For an external calibration curve, the uncertainty of the interpolated concentration, $\mathrm{S}_{\mathrm{X}_{0}}$, was calculated using:

$$
\mathrm{S}_{\mathrm{X}_{0}}=\frac{\mathrm{S}_{\mathrm{y} / \mathrm{x}}}{\mathrm{b}} \sqrt{\frac{1}{\mathrm{~m}}+\frac{1}{\mathrm{n}}+\frac{\left(\mathrm{y}_{0}-\overline{\mathrm{y}}\right)^{2}}{\mathrm{~b}^{2} \sum_{\mathrm{i}}\left(\mathrm{x}_{\mathrm{i}}-\overline{\mathrm{x}}\right)^{2}}}
$$

Where $\mathrm{m}$ is the number of replicate measurements on each calibration standard, $\mathrm{y}_{0}$ is the experimental value from which the concentration of $\mathrm{x}_{0}$ is to be determined, and the other variables have the same definition as those in Equation (12). Using either $\mathrm{S}_{\mathrm{y} / \mathrm{x}}$ or $\mathrm{S}_{\mathrm{X}_{0}}$ as the standard deviation, the \%RSD can be calculated using Equation (15). Confidence limits were then calculated using Equation (16). 


$$
\begin{gathered}
\text { \%RSD }=100 \% \frac{\text { Standard Deviation of Calculated Conc. }(\mathrm{ppm})}{\text { Mean of the Calculated Conc. }(\mathrm{ppm})} \\
\mathrm{x}_{\mathrm{E}} \pm \mathrm{t}_{(\mathrm{n}-2)} \mathrm{S}_{\mathrm{x}_{\mathrm{E}}} \text { or } \mathrm{x}_{0} \pm \mathrm{t}_{(\mathrm{n}-2)} \mathrm{S}_{\mathrm{x}_{0}}
\end{gathered}
$$

In this equation, $\mathrm{n}$ is once again the number of calibration standards and $\mathrm{t}$ is the Student's t-distribution. The limits of detection (LOD) for each element can be calculated using Equation (17).

$$
\mathrm{LOD}=\frac{3 \times \mathrm{S}_{\mathrm{y} / \mathrm{x}}}{\mathrm{b}}
$$

Equation (17) 


\section{CHAPTER 3. Optimization of Sample Preparation for LA-ICP-MS}

The section will cover the optimization and results of the different sample preparation methods and calibration strategies used for LA-ICP-MS analyses. The methods used for $k_{0}$ INAA was previously optimized and published [6] and will not be discussed.

An accurate quantitative method with minimum sample preparation steps was ideal. Different approaches to preparing the samples were evaluated. The \%bias and $\%$ RSD for each method is summarized after each section where available.

\subsection{Tape Mounting}

\subsubsection{Sample Preparation}

The easiest sample preparation involved simply taking the milk powder and spreading it evenly over a piece of double sided tape attached to a glass microscope slide. This method proved troublesome when it came to quantitative analysis. The transient signals for this sample preparation were bumpy and inconsistent, however the use of an internal standard would account for differences in mass ablated. It was thought that an element that is of high concentration in milk, $\mathrm{Ca}$ or $\mathrm{K}$, could be used as an internal standard. However, a review of the literature showed that the concentration of $\mathrm{Ca}$ and $\mathrm{K}$ in milk powder can change [9]. Spiking an internal standard also caused problems. If the volume of the internal standard was too small, it was difficult to homogenize the sample by just vortexing. Increasing the volume of the spike caused either the formation of a thick solution or small round balls. After drying, both would harden and had to be crushed to form a spreadable powder. 


\subsubsection{Results}

However, using single point calibration with IAEA-155 as the calibrator had a very poor \%bias for all elements, greater than $100 \%$, data not shown. ${ }^{85} \mathrm{Rb}$ and ${ }^{88} \mathrm{Sr}$ had negative concentrations. The transient signals for these elements, ${ }^{85} \mathrm{Rb}$ and ${ }^{88} \mathrm{Sr}$, looked to be below the LOD, Figure 13, which could account for why these elements gave a negative concentration. Even with the use of an internal standard, the \%RSD for the tape mounting method ranged from $15-39 \%$. 


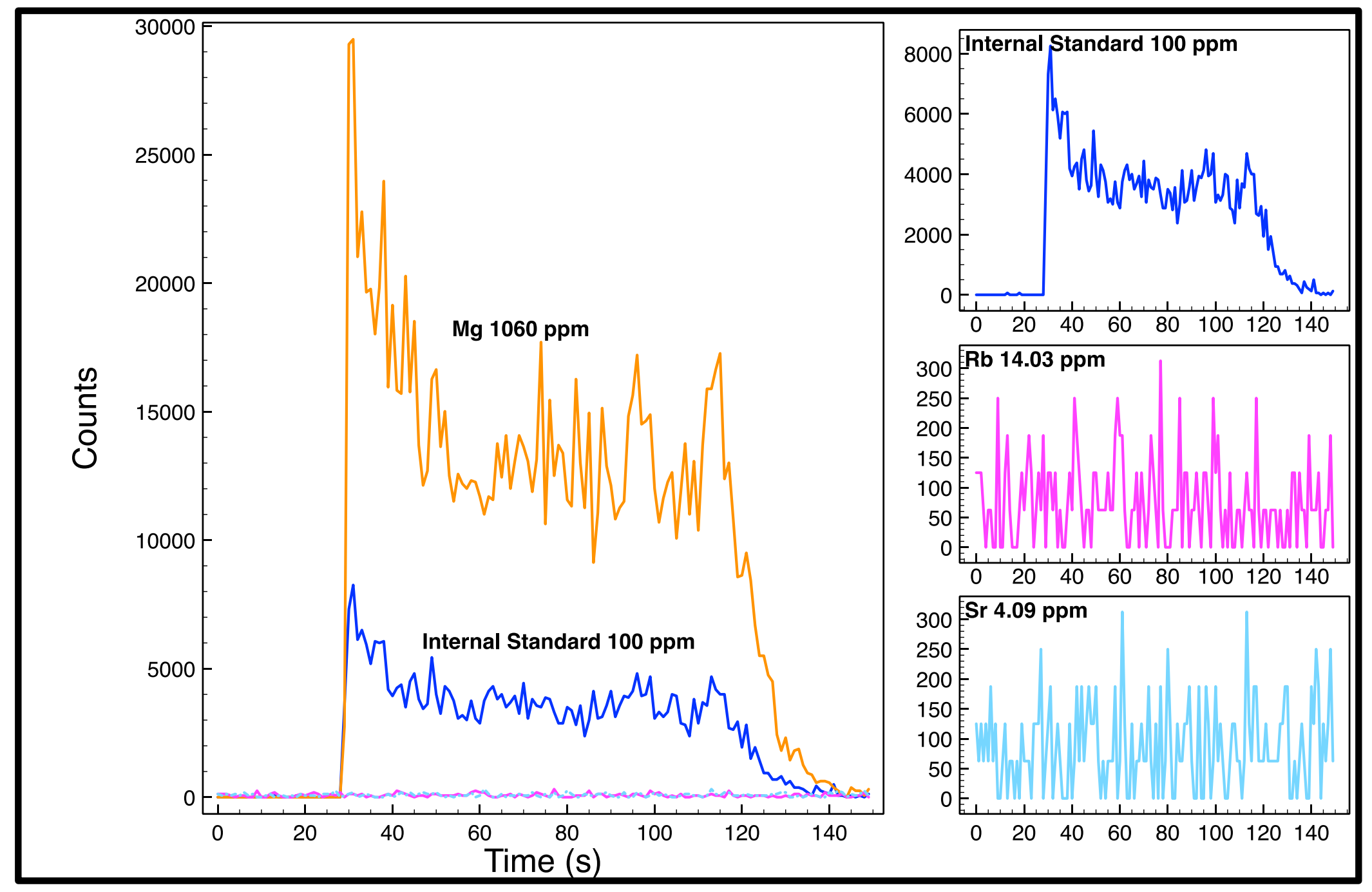

Figure 13 - Transient signal for the tape sample preparation 


\subsection{Dried Milk Discs}

\subsubsection{Sample Preparation}

The next sample preparation involved measuring the harden disc that formed during the tape sample prep analysis. To create this disc, $0.5 \mathrm{~g}$ of milk powder was weighed into the screw cap of a digestion vessel (Environmental Express, Charleston, South Carolina). An internal standard was spiked as well as $0.5 \mathrm{~mL}$ of high purity water. The container was attached to the lid and the mixture was vortexed upside down, which caused the formation of a thick milk solution. The solution was left to dry in an oven overnight, Figure 14. The ablation chamber of the laser was not large enough to fit the lid, so a large piece of the sample was removed and placed on double-sided tape. Focusing the laser was difficult because of the uneven surface.

\subsubsection{Results}

Single point calibration with GLITTER with IAEA-155 as the calibrator was tested, Table 5. The \%bias was less than $10 \%$ for most elements except $\mathrm{Sr}(30 \%), \mathrm{Zn}$ (23\%), and K (21\%). The \%RSD was less than $10 \%$ for all elements except Zn. Problems occurred, however, when trying to prepare other milk samples. Some samples formed a ball when $0.5 \mathrm{~mL}$ of water was added. More water was added until the sample formed a solution. However, when these samples were dried, the resulting disc was very thin or non-existent and difficult to remove from the lid, Figure 14. 
Table 5 - Results for IAEA-153 using the dried disc method with single point calibration

\begin{tabular}{|cccc|}
\hline Element & $\begin{array}{c}\text { Recommended Value IAEA-153 } \\
(\mathrm{ppm}, 95 \% \text { Confidence Interval })\end{array}$ & \% Bias & \%RSD \\
\hline $\mathrm{Ca}$ & $12870(12540-13170)$ & 2.4 & 9.0 \\
$\mathrm{Mg}$ & $1060(1000-1150)$ & 7.9 & 3.3 \\
$\mathrm{~K}$ & $17620(16480-18760)$ & 21.2 & 7.6 \\
$\mathrm{Rb}$ & $14.03(12.27-16.10)$ & 8.5 & 8.8 \\
$\mathrm{Na}$ & $4180(3870-4440)$ & 3.9 & 8.0 \\
$\mathrm{Sr}$ & $4.09(3.49-4.73)$ & 32.2 & 7.2 \\
$\mathrm{Zn}$ & $39.56(37.66-41.23)$ & 22.5 & 36.0 \\
\hline
\end{tabular}

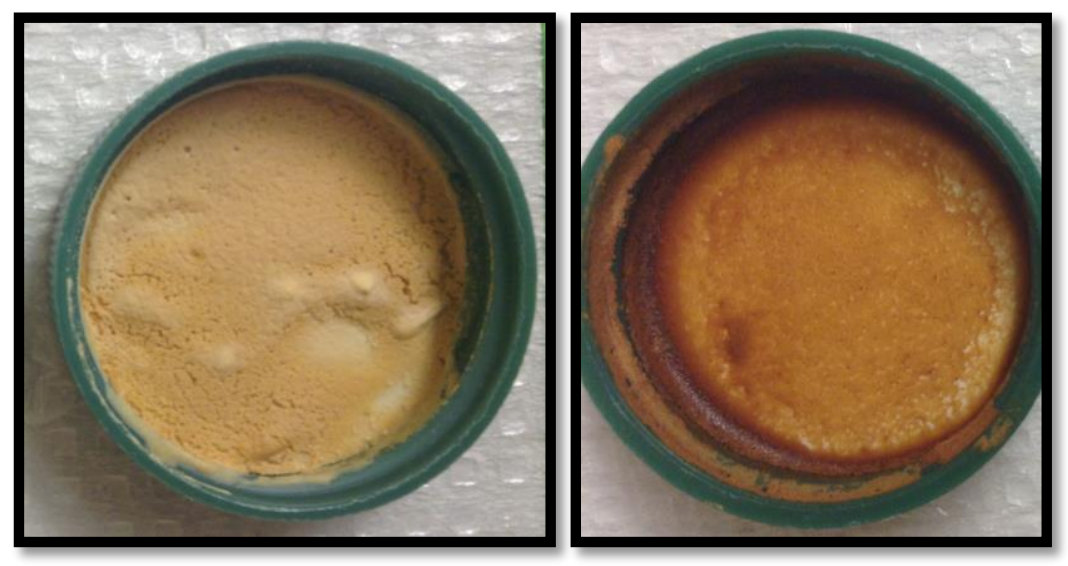

Figure 14 - Dried discs samples for IAEA-153 and IAEA-155 after addition of internal standard and $0.5 \mathrm{~mL}$ of water and drying overnight 


\subsection{Dried Smears on Glass}

\subsubsection{Sample Preparation}

To help combat this problem, the next sample preparation involved creating a dried milk smear on a glass slide instead of a dried disc. A sample of $0.5 \mathrm{~g}$ of milk powder was weighed out. An internal standard and $1.0 \mathrm{~mL}$ of high purity water was added. The sample was vortexed. The solution was spread over a microscope slide using a cover slip. Care was taken to not spread the solution too thin. The microscope slides were sent to dry on a Hot Block overnight.

The laser parameters were similar to those used for by the Almirall research group for the analysis of paint and ink: 40\% energy, $1 \mathrm{~mm}$ line, $25 \mu \mathrm{m} / \mathrm{s}, 10 \mathrm{~Hz}, 190 \mu \mathrm{m}$ spot size. During ablation of the sample, the dried smear would flake off in large pieces, which left the laser firing directly onto the glass slide. These flakes would cause a large bump in the transient signal if the pieces were carried to the plasma. The laser energy and frequency were reduced and scan rate increased, but the problem was not solved. No quantitative analysis was done with the data.

\subsection{Dried Spots}

For the dried spot sample preparation, instead of spreading the milk solution on glass, pre-cut Whatman filter paper and plastic were used. This is a sample preparation that was used for LA-ICP-MS analysis of broth samples [41].

\subsubsection{Selecting a Substrate and Volume Size}

A sample of $0.1 \mathrm{~g}$ of milk powder was weighed out and placed in polypropylene test tubes with snap caps (Fischer Scientific, Pittsburgh PA) and $2.0 \mathrm{~mL}$ of high purity 
water was added to allow the solution to be pipetted. The solutions were vortexed until all of the milk powder dissolved.

Circular discs of $16 \mathrm{~mm}$ and $6 \mathrm{~mm}$ were cut from Whatman 42 filter paper, Whatman 542 filter paper, and disposable polystyrene beakers using a hole-punch. These circular discs were attached to glass microscope cover slides using double sided tape. For the $13 \mathrm{~mm}$ discs, only one was attached to each slide. For the $6 \mathrm{~mm}$ discs, four discs per slide.

A micropipette was used to deposit $10 \mu \mathrm{L}, 20 \mu \mathrm{L}, 30 \mu \mathrm{L}$ of the milk solution onto the center of each $6 \mathrm{~mm}$ disc and $100 \mathrm{uL}, 300 \mu \mathrm{L}$, and $500 \mu \mathrm{L}$ on the $16 \mathrm{~mm}$ discs. Care was taken not to create air bubbles, but bubbles occurred especially with the higher volume spikes. The $30 \mu \mathrm{L}$ spikes would sometimes overflow from the Whatman filters partially onto the double-sided tape. The same would happen with $500 \mu \mathrm{L}$ spikes on the Whatman filters, Figure $15 \mathrm{C}$. The polystyrene was able to hold the larger volume spikes, Figure $16 \mathrm{C}$. The samples were left overnight to air dry. The dried spots on the polystyrene were more uniform in color than the dried spots on the Whatman filter papers, Figure 17. Discoloration could be seen on the filter papers in places where bubbles formed, usually the center. 


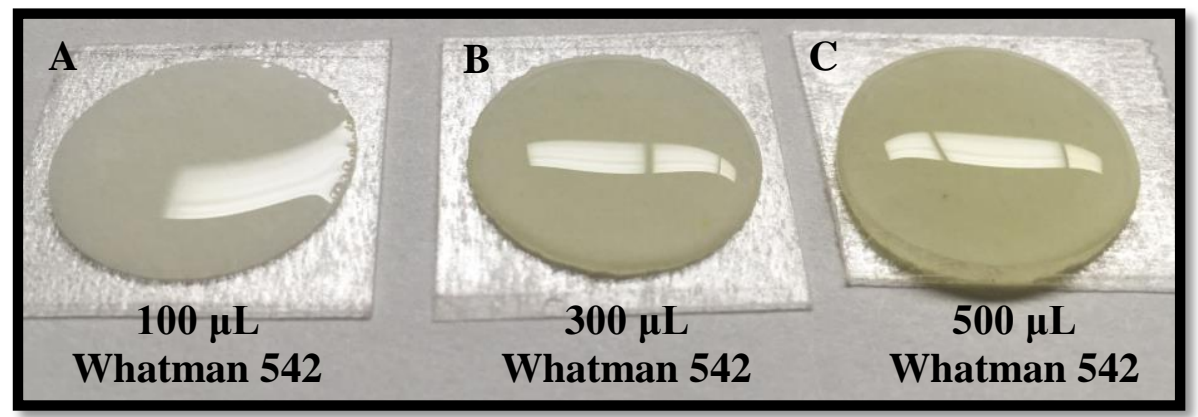

Figure 15 - Different volume spikes on $16 \mathrm{~mm}$ dried spots

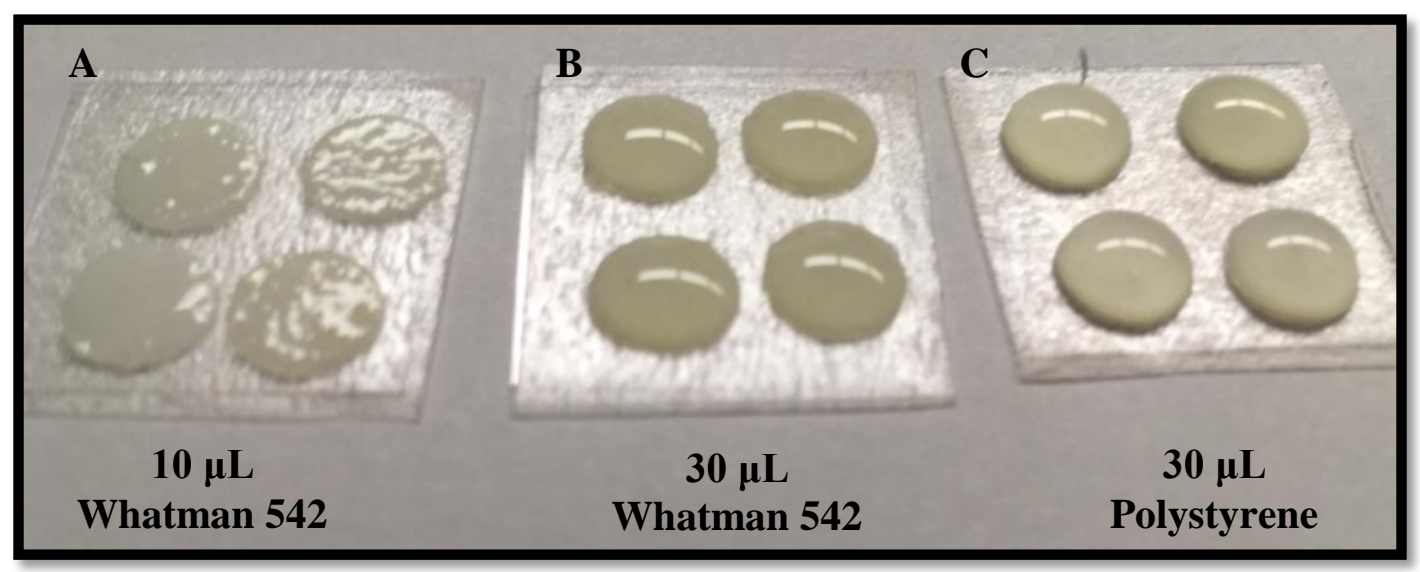

Figure 16 - Different volume spikes on $6 \mathrm{~mm}$ dried spots

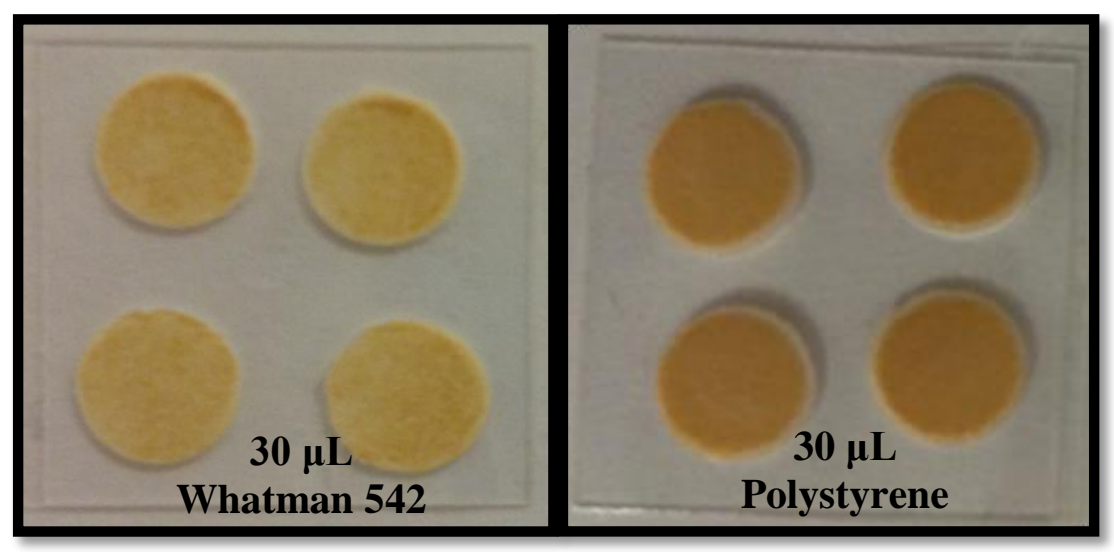

Figure 17 - Comparison of dried spots on Whatman paper and polystyrene 
The laser parameters were optimized to limit the amount of substrate contribution. A 3D microscope was used to visualize cross sections of each ablation line. First the laser energy was optimized. The frequency was set at $10 \mathrm{~Hz}$, scan rate at $100 \mu \mathrm{m} / \mathrm{s}$, and spot to $190 \mathrm{um}$. The laser energy started at 50\% and was increased to $75 \%$ and $100 \%$. None of the ablation lines penetrated through the Whatman 542 filter paper or polystyrene. However, for the samples deposited on polystyrene, flaking of the samples occurred during analysis like what was seen with the glass smears. The laser coupled nicely with the samples deposited on the Whatman paper, with the Whatman 542 being slightly thicker and able to withstand harsher parameters. The laser energy was set at $100 \%$ and the scan rate was decreased to $50 \mu \mathrm{m} / \mathrm{s}$ and $25 \mu \mathrm{m} / \mathrm{s}$. At $25 \mu \mathrm{m} / \mathrm{s}$ the laser ablated through the filter paper is some areas. The \%RSDs were evaluated for all tested combinations. The parameters that produced the lowest \% RSDs were $100 \%$ energy, $6 \mathrm{~mm}$ or $16 \mathrm{~mm}$ line across the diameter of the Whatman 542 disc, $50 \mu \mathrm{m} / \mathrm{s}, 10 \mathrm{~Hz}, 190 \mu \mathrm{m}$ spot size.

The analysis time for the $16 \mathrm{~mm}$ disc was over twice as long as the $6 \mathrm{~mm}$. Though a larger volume could be pipetted, no other advantages were seen with using $16 \mathrm{~mm}$ over $6 \mathrm{~mm}$ discs. The smaller volumes had a better \%RSD than the larger ones. This could be caused by some of the volume spilling off of the discs. Quantitative analysis with $10 \mu \mathrm{L}$ pipetted on $6 \mathrm{~mm}$ Whatman 542 discs was further evaluated.

\subsubsection{External Calibration Curve}

\subsubsection{Sample Preparation}

An external calibration curve was created using ICP-MS element standards to create calibration solutions in high purity water. For the samples, an aliquot of $0.1 \mathrm{~g}$ of 
milk powder was weighed out. An internal standard and $2.0 \mathrm{~mL}$ of high purity water was added. Care was taken to reduce the amount of acid added to the milk powder samples to avoid precipitation of the proteins. An aliquot of $10 \mu \mathrm{L}$ of the solution was pipetted on the $6 \mathrm{~mm}$ Whatman 542 discs. The sample was left overnight to air dry.

\subsubsection{Results}

The calibration curves were very linear for all elements; $\mathrm{R}^{2}$ was greater than 0.99 , Figure 18. However, the calculated concentrations for IAEA-153 had high bias, Table 6. The high bias was thought to be caused by differences in the laser sample/ standard interactions (matrix effects). To see if this bias could be improved, the standard addition method was tested. 

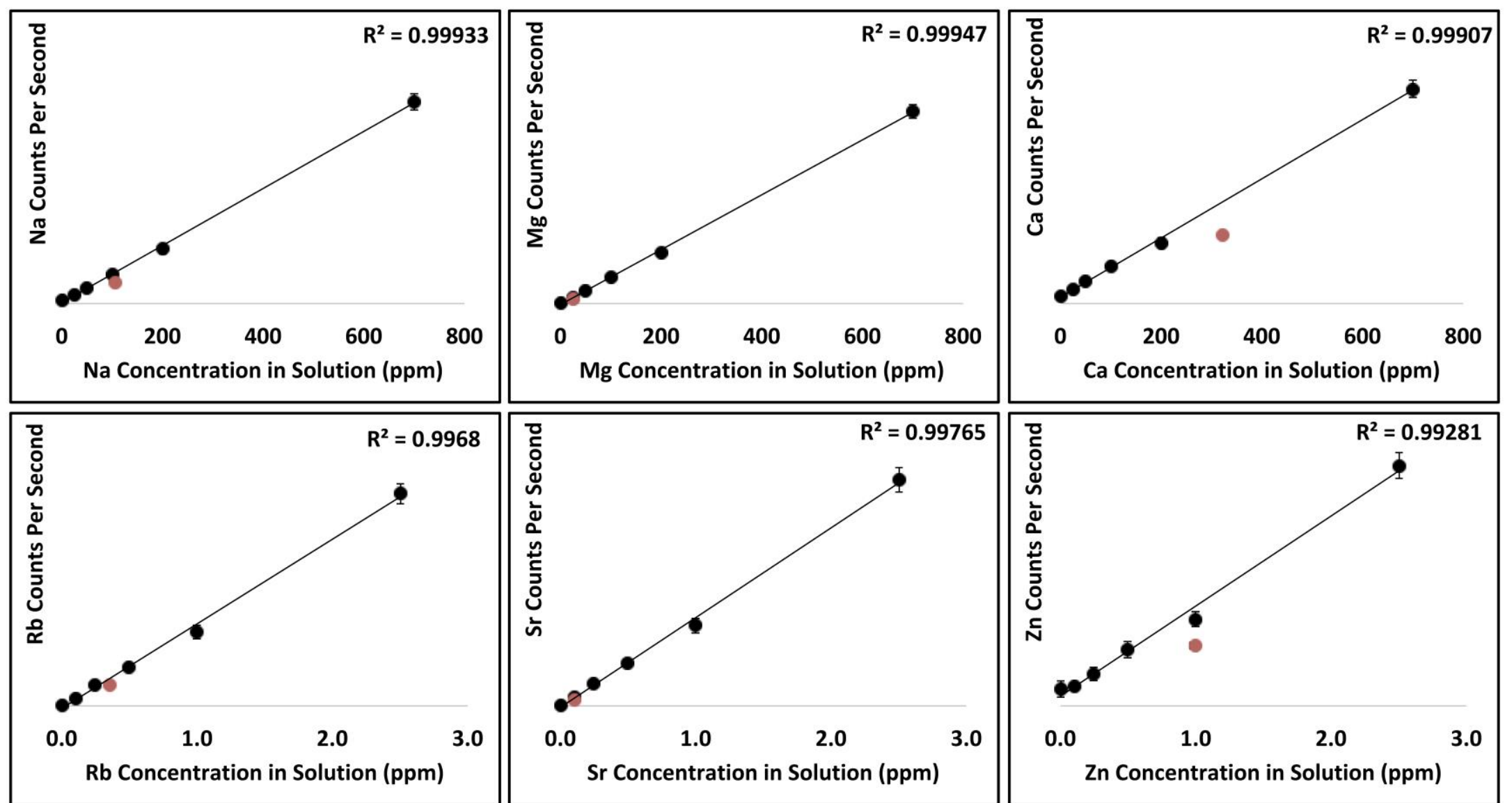

Figure 18 - External calibration curves for $\mathrm{Na}, \mathrm{Mg}, \mathrm{Ca}, \mathrm{Rb}, \mathrm{Sr}$, and $\mathrm{Zn}$ using ICP-MS standards and the dried spot sample preparation method; the $y$ error bars represent \pm 1 standard deviation. The red data points represent IAEA-153 and the measured counts per second. The $x$ error bars on the red data points represent $\pm 1 S_{x 0}$ 


\subsubsection{Standard Addition}

The problem with external calibration was thought to be caused by matrix effects between the milk powder samples and calibration standards. To determine if these matrix effects could be reduced or eliminated, a standard addition curve was created for each sample.

\subsubsection{Sample Preparation}

Elements of interest ( $\mathrm{Na}, \mathrm{Mg}, \mathrm{Ca}, \mathrm{Zn}, \mathrm{Rb}$, and $\mathrm{Sr}$ ), an internal standard, and $2 \mathrm{~mL}$ of high purity water (resistivity of $18.2 \mathrm{M} \Omega \mathrm{cm}$ ) were added to each sample. Samples were mixed thoroughly with a vortex touch mixer (Fischer Scientific, Pittsburgh PA). A micropipette was used to deposit $10 \mu \mathrm{L}$ of each solution onto the center of a Whatman disc. The samples were left to air-dry overnight.

\subsubsection{Results}

The standard addition curves for IAEA-153, have a $\mathrm{R}^{2}$ greater than 0.99 for all elements, Figure 19. However, the calculated \%bias was still higher than the desired $10 \%$ for most elements, Table 6 . The high bias could be the result of the casein proteins precipitating out of solution with the addition of the element standards. Interestingly, the measurement standard deviations are present on all data points, but they are usually small and hardly visible above the point. On exception is $\mathrm{Zn}$. Zinc has very large measurement standard deviations. An examination of the $\mathrm{Zn}$ transient signal did not reveal any information. No explanation can be given at the present time for why the $\mathrm{Zn}$ measurement standard deviations were high. 

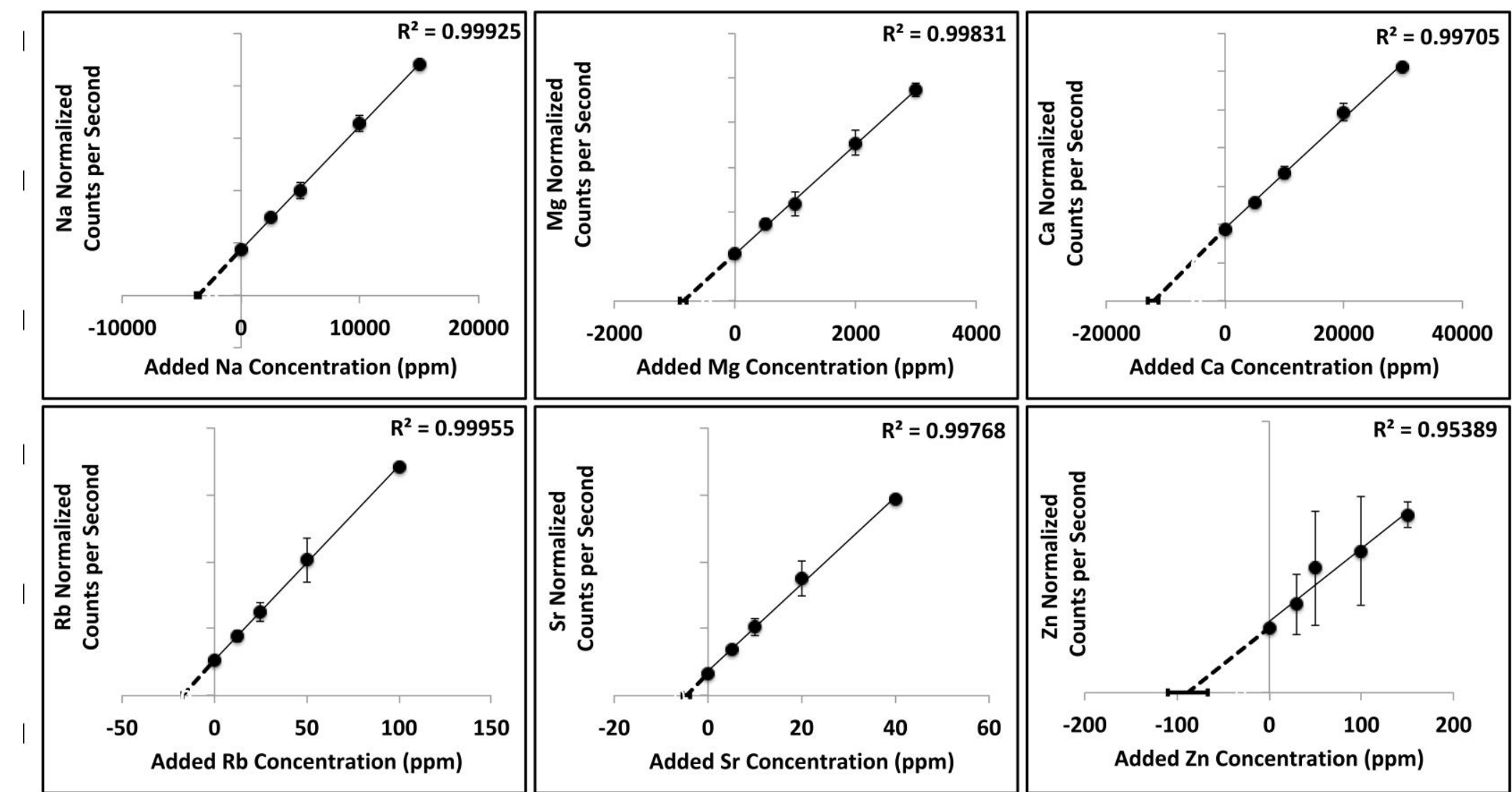

Figure 19 - Standard addition curves for $\mathrm{Na}, \mathrm{Mg}, \mathrm{Ca}, \mathrm{Rb}, \mathrm{Sr}$, and $\mathrm{Zn}$ using IAEA-153 and the dried spot sample preparation; the y error bars represent \pm 1 standard deviation. The $\mathrm{x}$ error bars are the $\mathrm{x}$-intercept represent $\pm 1 \mathrm{~S}_{\mathrm{XE}}$ 


\subsubsection{External Calibration Curve using IAEA-153}

The standard addition curve that was created using IAEA-153 was tested as an external calibration curve, Figure 20. However, when examining commercial milk powder samples, all had concentrations similar to that of IAEA-153. Some samples fell below the lowest calibration point. Since adding element spikes to milk powders had an adverse effect for some samples, the sample preparation was no longer studied.

\subsubsection{Conclusions}

This section focused on three sample preparation methods: the tape method, dried disc method, and the dried spot method. Though these sample preparations were quick and easy, the results in terms of percent accuracy and precision did not meet the desired values. The dried spot method was one of the more accurate methods, however, the \%bias was higher than the desired $10 \%$ for external calibration. Attempts to reduce the \%bias such as creating standard addition curves were tested. The standard addition method performed well for the IAEA-153 standard with a \%bias less than $23 \%$ for all elements except $\mathrm{Zn}$. However, this method was unsuccessful for some samples. This is partially because the elemental solutions are in acid. When they were added to a milk solution, precipitation of what was assumed to be casein, resulted. Another shortcoming of the standard addition method was that the analysis of five standard addition standards was time consuming, 40 minutes for the complete analysis of one sample. 

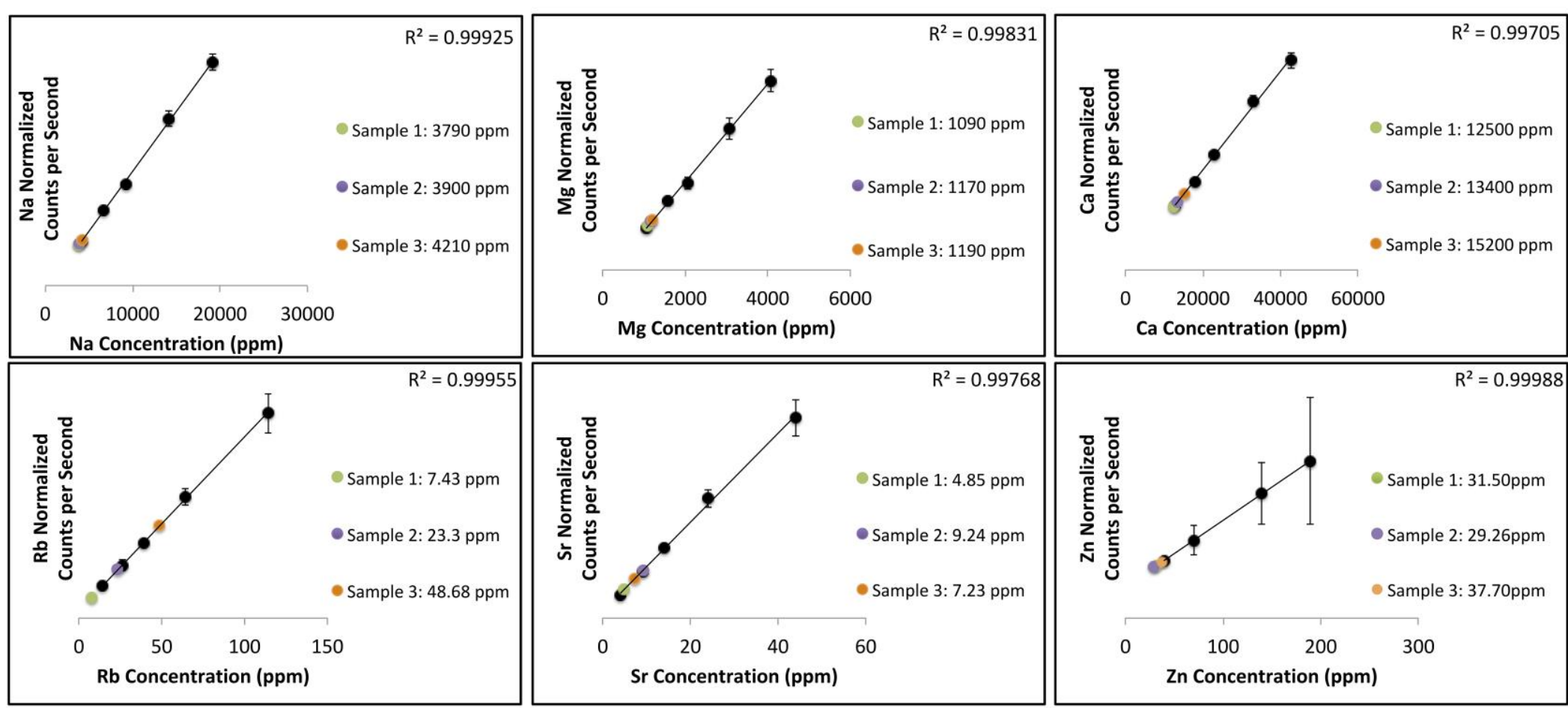

Figure 20 - External calibration curves for $\mathrm{Na}, \mathrm{Mg}, \mathrm{Ca}, \mathrm{Rb}, \mathrm{Sr}$, and $\mathrm{Zn}$ created using IAEA-153 and the dried spot sample preparation; the green, purple, and orange data points represent three different milk powder samples. As can be seen these data points fall below or on the lower end of the calibration curve for most elements. 
Table 6 - Shown are the calculated concentration, uncertainty, and bias for IAEA-153 using the different calibration strategies and the dried spot sample preparation method

\begin{tabular}{|c|c|c|c|c|c|c|c|}
\hline \multirow{2}{*}{\multicolumn{2}{|c|}{$\begin{array}{l}\text { Recommended Value IAEA-153 } \\
\text { (ppm, 95\% Confidence Interval) }\end{array}$}} & \multicolumn{3}{|c|}{$\begin{array}{c}\text { External Calibration } \\
\text { With Element Solutions }\end{array}$} & \multicolumn{3}{|c|}{ Standard Addition } \\
\hline & & $\begin{array}{c}\text { Conc. } \\
\left(\mathrm{X}_{0}, \mathrm{ppm}\right)\end{array}$ & $\begin{array}{l}\text { Uncertainty } \\
\left(S_{X_{0}}, \mathrm{ppm}\right)\end{array}$ & $\begin{array}{l}\text { Bias } \\
(\%)\end{array}$ & $\begin{array}{c}\text { Conc. } \\
\text { (XE, ppm) }\end{array}$ & $\begin{array}{l}\text { Uncertainty } \\
\left(S_{X_{E}}, \mathrm{ppm}\right)\end{array}$ & $\begin{array}{l}\text { Bias } \\
(\%)\end{array}$ \\
\hline Ca & $12870(12540-13170)$ & 8330 & 147 & -35 & 12100 & 892 & 6 \\
\hline Mg & $1060(1000-1150)$ & 872 & 117 & -18 & 858 & 857 & 19 \\
\hline $\mathbf{R b}$ & $14.03(12.27-16.10)$ & 11.3 & 0.9 & -19 & $\mathbf{1 7 . 3}$ & 0.8 & 23 \\
\hline $\mathrm{Na}$ & $4180(3870-4440)$ & 2770 & 128 & -33 & 3630 & 182 & 13 \\
\hline $\mathbf{S r}$ & $4.09(3.49-4.73)$ & 3.25 & 0.89 & -20 & 4.64 & 0.70 & 13 \\
\hline Zn & $39.56(37.66-41.23)$ & 22.0 & 1.5 & -44 & 88.7 & 21.9 & 124 \\
\hline
\end{tabular}




\subsection{Pelleting}

\subsubsection{Spiking Samples}

The sample preparation steps were optimized so that the internal standards and element spikes were homogenized in the milk. At first, the standard addition pellets were created so that the first pellet only contained the internal standard and the last pellet contained the highest concentration for each element. However, since the ICP-MS element solutions are in acid, this resulted in partial digestion of the highest calibration standard, which led to differences in how the laser coupled to the sample. In attempts to combat this, $0.8 \mathrm{M}$ nitric acid was added to all calibration standards in amounts so that the total volume of solution added was equivalent to the volume added to the highest calibration standard. This caused burning and partial digestion for some milk powder samples.

The next attempt involved mixing the concentration of the elements so that one pellet contained the highest concentration of one element and the lowest of another. Combinations were chosen so that all standard addition pellets received the same amount of solution without the need of additional $0.8 \mathrm{M}$ nitric acid. This solved the problem. 
Table 7 - The concentration of each element added to the each of the calibration pellets; to prevent acid digestion of the milk powders, the element spikes were spread out so each standard received the same spike volume. The lowest calibration standard for one element might be the highest calibration standard for another.

\begin{tabular}{|ccccc|}
\hline Element & $\begin{array}{c}\text { Calibration } \\
\text { Standard } 1 \\
(\mathrm{ppm})\end{array}$ & $\begin{array}{c}\text { Calibration } \\
\text { Standard 2 } \\
(\mathrm{ppm})\end{array}$ & $\begin{array}{c}\text { Calibration } \\
\text { Standard 3 } \\
(\mathrm{ppm})\end{array}$ & $\begin{array}{c}\text { Calibration } \\
\text { Standard 4 } \\
(\mathrm{ppm})\end{array}$ \\
\hline $\mathrm{Na}$ & 500 & 1000 & 2000 & 0 \\
$\mathrm{Mg}$ & 250 & 500 & 0 & 1000 \\
$\mathrm{Ca}$ & 500 & 1000 & 0 & 2000 \\
$\mathrm{Zn}$ & 60 & 30 & 10 & 0 \\
$\mathrm{Rb}$ & 20 & 10 & 5 & 0 \\
$\mathrm{Sr}$ & 10 & 5 & 2.5 & 0 \\
\hline
\end{tabular}

\subsubsection{Selecting an Internal Standard}

Figure 21 also shows the integration strategy for selecting the gas blank and signal ranges. The gas blank was subtracted from the signal. The blank-subtracted signal was normalized to the internal standards ( $\mathrm{K}$, Sc, and In). IAEA-153 was used to assess the performance of each internal standard. All three internal standards produced accurate concentrations for $\mathrm{Zn}, \mathrm{Rb}$, and $\mathrm{Sr}$. However, $\mathrm{K}$ performed the best for $\mathrm{Na}, \mathrm{Mg}$, and $\mathrm{Ca} . \mathrm{K}$ normalization also produced more linear calibration curves which resulted in smaller concentration uncertainties. In the end, all elements were normalized to $\mathrm{K}$ for this study. 


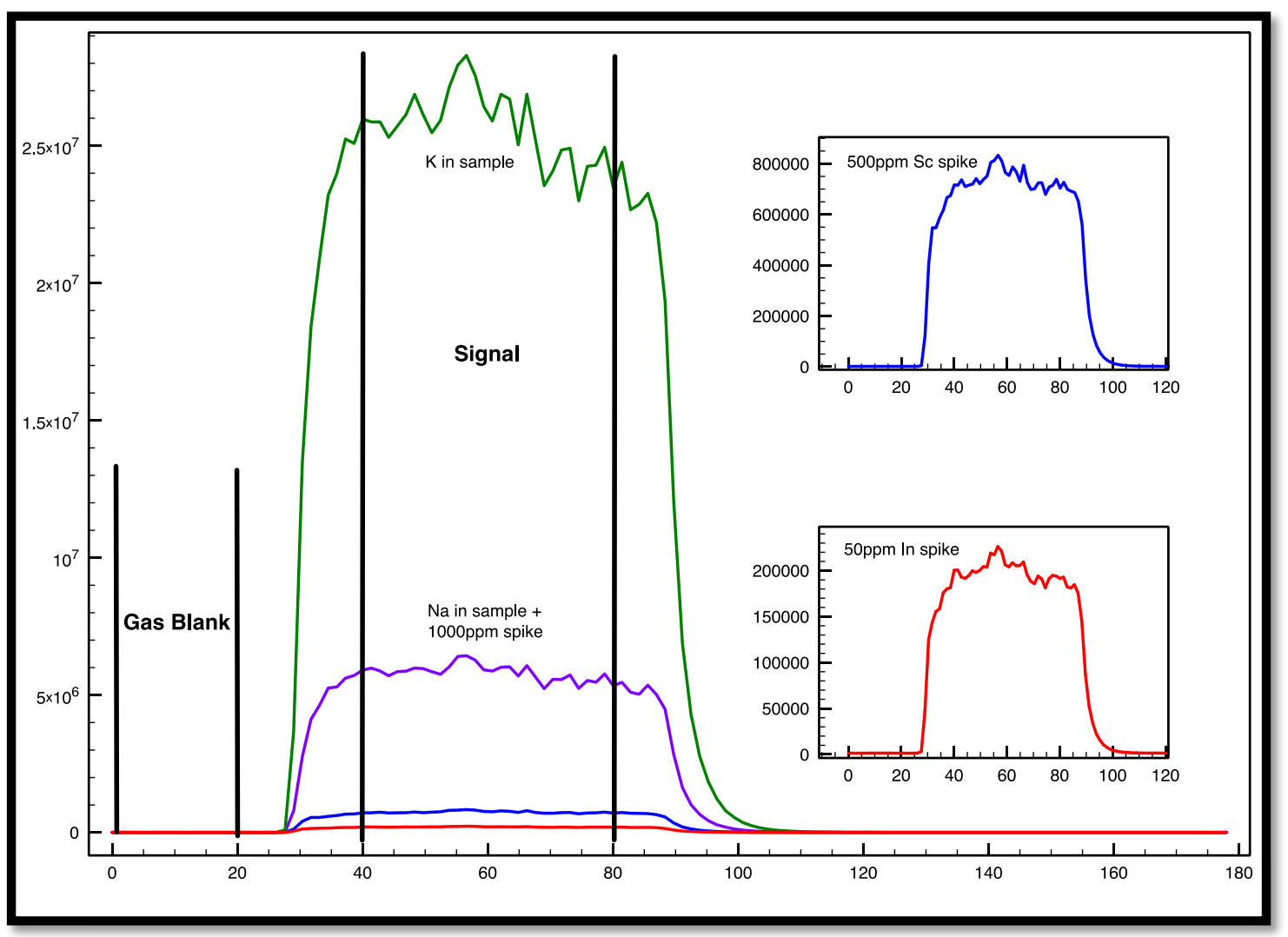

Figure 21 - LA-ICP-MS signal for pellets

\subsubsection{Drying Samples}

The next step in sample preparation was drying the spiked powders to remove the liquid added by the element solutions. To dry the samples, a Hot Block and oven were tested at temperatures ranging from $60-100^{\circ} \mathrm{C}$ and times ranging from 4- 24 hours.

The samples were placed in either the Hot Block or oven immediately after the addition of solution spikes. To optimize the temperature, the samples were heated for 4 hours and visually analyzed. There was a slight darkening of the powders after heating but no other issues occurred. The samples were weighed after 4 hour intervals until two consecutive readings differed by no more than $0.005 \mathrm{mg}$. The optimized parameters of $80^{\circ} \mathrm{C}$ for up to 12 hours were selected because this combination was able to completely 
dry the sample without burning it. No differences where seen between drying the samples in the oven and Hot Block. However, the Hot Block was able to securely contain the sample vials without fear of accidentally knocking samples over.

\subsubsection{Milling Studies}

The next step in sample preparation was milling. Two types of mill jars were tested, Teflon and tungsten, along with various milling times ( 2 minutes, 5 minutes, 10 minutes, 15 minutes, 30 minutes) and frequencies $(2 \mathrm{~Hz}, 5 \mathrm{~Hz}, 7.5 \mathrm{~Hz}, 10 \mathrm{~Hz}, 25 \mathrm{~Hz}, 30$ $\mathrm{Hz}$ ) to determine the parameters needed to homogenize the element spikes. Differences were seen in milling milk samples of different densities and consistencies. Commercial nonfat and skim milk were able to tolerate longer mill times and higher frequencies. Commercial full cream samples as well as some of the samples dried in a laboratory would adhere to the sides of the mill jars at the higher mill times and frequencies, Figure $22 \mathrm{~A}$. However at lower mill times and frequencies, these samples were not homogenized and large clumps were visible, Figure $22 \mathrm{~B}$. The use of Teflon milling jars was not able to prevent the samples from adhering to the sides of the jars, and samples that did not stick to the walls were not homogenized even at $30 \mathrm{~Hz}$ for 15 minutes. 

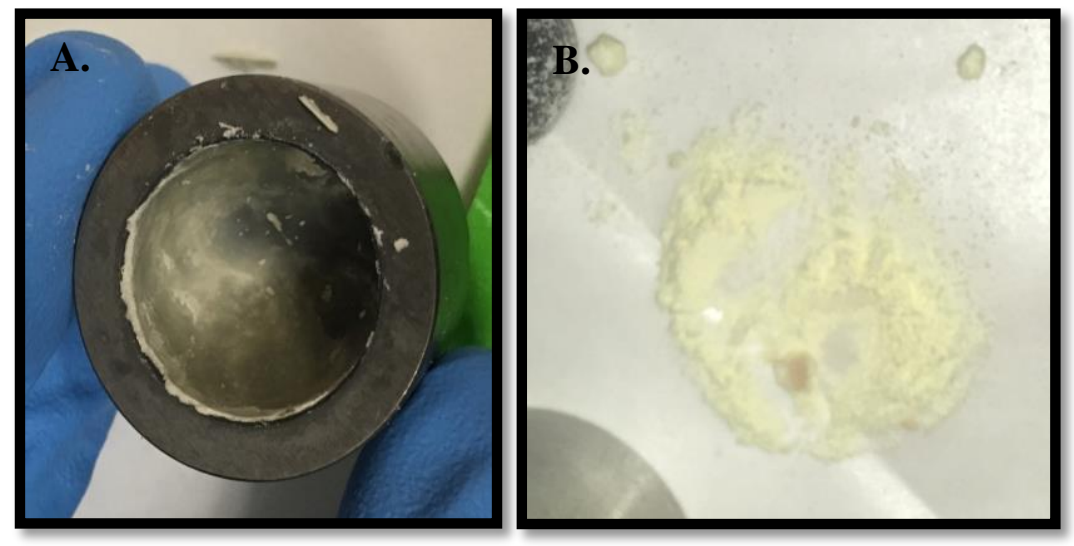

Figure 22 - A. Sample adhering to mill jar because the frequency was too high and/or the length of milling was too long B. Clumps of milk powder that were not milled long enough at a high enough frequency

Tungsten milling jars were further tested to find parameters that worked for both types of samples. Both types of samples could be milled at $25 \mathrm{~Hz}$ for 5 minutes, but this was not long enough to homogenize the Sc and In internal standards in the commercial nonfat samples, Figure 23 B. The length of milling was increased until the internal standards were homogenized in both samples.

Figure 21 shows the transient signal for $\mathrm{K}, \mathrm{Na}, \mathrm{Sc}$, and In after milling for 15 minutes. $\mathrm{K}$ was not added to any of the samples so the signal shown represents the $\mathrm{K}$ content in the milk powder. $\mathrm{Na}$ is naturally present in milk powders and is also an element that was spiked in the samples. The signal depicted is a combination of the natural $\mathrm{Na}$ and a $1000 \mathrm{ppm}$ spike. Sc and In are not normally present in milk or are present in a very small amount; these elements are spiked into the milk samples at 500 ppm and $50 \mathrm{ppm}$ respectively. The element transient signals all follow the same trend indicating that the element spikes are homogenized in the sample. 

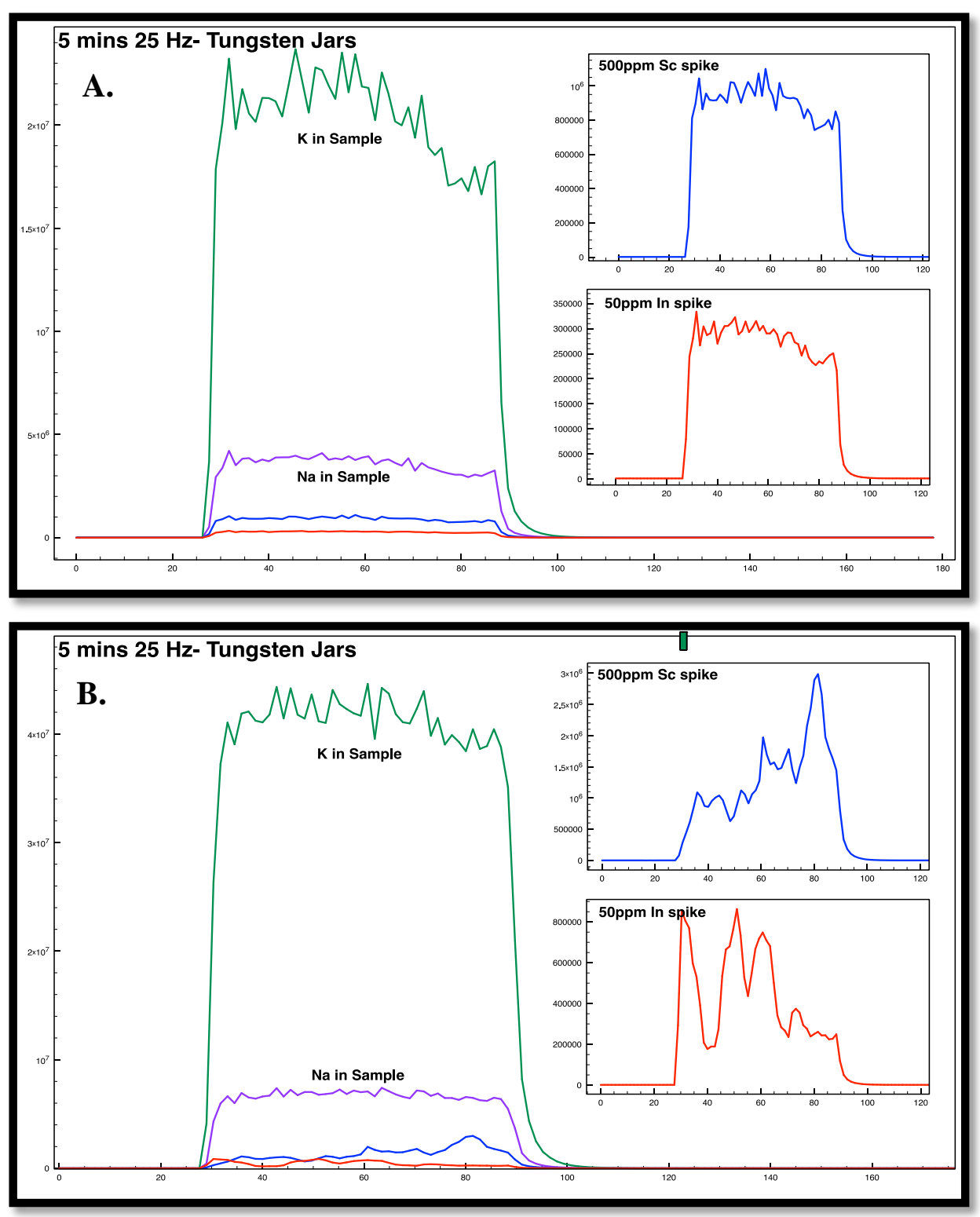

Figure 23 - A. is the transient signal for a commercial full cream milk powder sample with a homogenized internal standard $B$. is the transient signal for a commercial nonfat milk powder sample where the internal standard was not homogenized in the sample

\subsubsection{Quantitative Analysis of Pellets}

A spot size of $190 \mu \mathrm{m}$ was selected to help account for homogeneity issues. Laser energy and frequency were optimized to ensure that the pellet surface was efficiently ablated and that the pellet surface was not fractured which would lead to non- 
reproducible sampling. Different combinations were tested and the pellets were examined under a 3D microscope. The number of shots was changed with the frequency so that a sample signal of at least $60 \mathrm{~s}$ was produced in the transient signal.

The NW 213 nm laser coupled well with the milk powder samples. Figure 24 shows the milk powder sample about analysis. No fracturing of the surface occurred with the maximum laser energy and frequency, $100 \%$ energy and $10 \mathrm{~Hz}$. These parameters created a uniform crater that was $142.5 \mu \mathrm{m}$ deep, Figure 24. At these parameters the transient signal remained relatively constant.

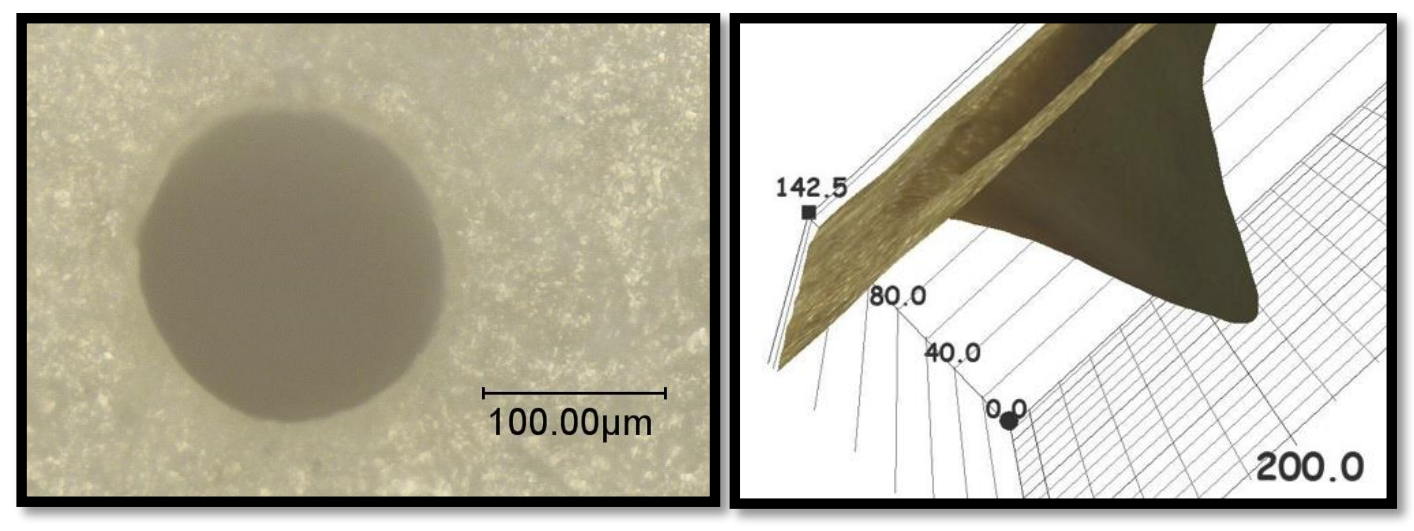

Figure 24 - Craters created using the optimized laser parameters visualized using a Keyence 3D microscope

\subsubsection{Single Point Calibration}

For each sample, 0.5 grams were weighed out and spiked with 10000 ppm Scandium (Sc) single element ICP-MS standard to a final concentration of $500 \mathrm{ppm}$. The samples were dried overnight using the Hot Block. Once dried, the samples were homogenized using the tungsten high-speed ball mixer mill and pressed into $13 \mathrm{~mm}$ pellets. 
The laser parameters were optimized as: $100 \%$ energy, $10 \mathrm{~Hz}, 190 \mathrm{um}$, and a 60 second dwell time. Background subtraction, integration of the transient signal, and calibration to IAEA-155 was performed in GLITTER.

The RSDs for this method ranged from 1-11\%, which were close to the desired RSD of $10 \%$. Zn was the element with the highest RSD. However, the \%bias for IAEA153 using this method was higher than desired for $\mathrm{Sr}(25 \%)$ and $\mathrm{Zn}(23 \%)$. The use of other internal standards and alterations to the ICP parameters such as a longer dwell time did not improve the results. Therefore additional calibration techniques were tested.

\subsubsection{External Calibration}

An external calibration curve was created by mixing the IAEA-155 whey powder with the casein for a total mass of 0.5 grams. The mixtures tested were $0 \%, 25 \%, 50 \%$, 75\%, and 100\% IAEA-155 whey powder. Yttrium (Y) single element ICP-MS standard solution was added ("spiked") as an internal standard to each sample to a final concentration of $1000 \mathrm{ppm}$.

To prepare the pellets, the spiked IAEA-155/casein samples were homogenized and pulverized to a fine powder using a high speed ball mixer mill (Glenmills, Clifton, NJ, USA) with a tungsten carbide ball and cups at $25 \mathrm{~Hz}$ for 10 minutes and pressed into pellets of $13 \mathrm{~mm}$ in diameter in a $13 \mathrm{~mm}$ stainless steel die at $15000 \mathrm{psi}$ for 2 minutes under vacuum (Carver Benchtop Pellet Press, IN, USA).

During analysis, it was noted that the $100 \%$ IAEA-155 and $100 \%$ casein samples did not ablate the same as the mixtures. The calibration curves also showed these points 
falling off the trend line. For the next test, these samples were replaced with a $90 \%$ IAEA-155 and 10\% IAEA-155.

The laser parameters were optimized as: $100 \%$ energy, $10 \mathrm{~Hz}, 190 \mathrm{um}$. The data were integrated with Plot and normalized to Y. Figure 25, shows the calibration curves. The red point represents the actual concentration of IAEA-153. For most elements, this point falls right on the calibration curve. For $\mathrm{Rb}$ and $\mathrm{Sr}$, this point falls slightly to the left, which indicates that the calibration curve is overestimating the concentration. Figure 25 shows the \%bias and uncertainty for each element. This method performed very well. Most elements had a \%bias and uncertainty less than $10 \%$. However $\mathrm{Sr}$, which has been a useful element in the geolocation of other foodstuffs had a \%bias of 33\%. Since the single point calibration and this external calibration curve rely on the use of whey protein (IAEA-155) to determine the concentration in a milk standard, it could be possible that both methods are suffering slightly from matrix effects caused by differences in the way the laser couples to each of these samples. Therefore, a standard addition curve was tested next to see if it could produce better results. 


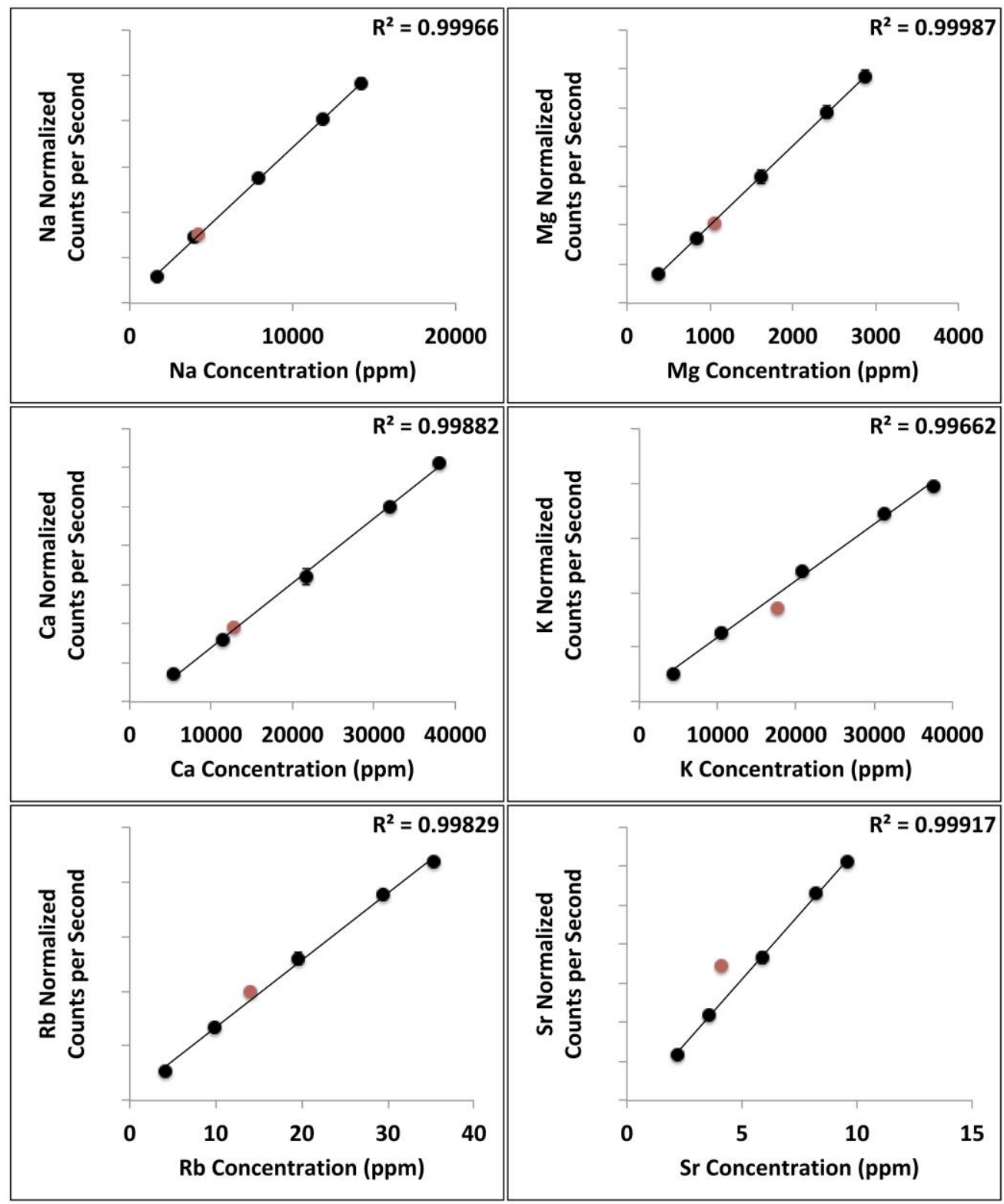

Figure 25 - External calibration curves for $\mathrm{Na}, \mathrm{Mg}, \mathrm{Ca}, \mathrm{K}, \mathrm{Rb}$, and $\mathrm{Sr}$ generated by diluting IAEA155 with casein. The normalized, background subtracted CPS are plotted vs the concentration in the pellet. The $y$ error bars represent \pm 1 standard deviation. The red data points represent the reported concentration for IAEA-153 at the CPS determined after data analysis. he $x$ error bars are the red data points represent $\pm 1 S_{x} 0$ 


\subsubsection{Standard Addition}

For the creation of the standard addition curves, single element ICP (CPI International, Santa Rosa, CA) and ICP-MS (Ricca Chemical Company, Pocomoke City, MD) standard solutions of $\mathrm{Zn}, \mathrm{Rb}, \mathrm{Sr}$, and $\mathrm{In}$ at $1000 \mu \mathrm{g} / \mathrm{mL}$ and $\mathrm{Na}, \mathrm{Mg}, \mathrm{Ca}, \mathrm{Sc}$ at $10,000 \mu \mathrm{g} / \mathrm{mL}$ were used.

Four representative samples of approximately $0.5 \mathrm{~g}$ of milk powder were accurately weighed to a precision of $0.1 \mathrm{mg}$ into $17 \times 100 \mathrm{~mm}$ polypropylene tubes. When sample mass was scarce, only $0.15 \mathrm{~g}$ of powder was used. Scandium and indium single element ICP-MS standard solutions were added ("spiked") as internal standards to each sample to a final concentration of 500 and 50 ppm, respectively.

Since the ICP element standards used are in nitric acid, partial digestion of the milk powders could occur. Calibration solutions were prepared that allowed the same amount of solution (160 $\mu \mathrm{L}$ for $0.5 \mathrm{~g})$ to be added to each sample. Samples were mixed thoroughly with a vortex touch mixer (Fischer Scientific, Pittsburgh, PA) and dried in air at $80^{\circ} \mathrm{C}$ in a PTFE-coated graphite Hot Block (Environmental Express, Charleston, SC) for up to $12 \mathrm{hrs}$.

To prepare the pellets, the dried milk powder samples were homogenized and pulverized to a fine powder using a high speed ball mixer mill (Glenmills, Clifton, NJ, USA) with a tungsten carbide ball and cups at $25 \mathrm{~Hz}$ for 15 minutes. For samples with a mass of $0.5 \mathrm{~g}$, the samples were pressed into pellets of $13 \mathrm{~mm}$ in diameter in a $13 \mathrm{~mm}$ stainless steel die at 4 tons for 2 minutes (Carver Benchtop Pellet Press, IN, USA). For samples with a mass of $0.15 \mathrm{~g}$, the samples were pressed into pellets of $6 \mathrm{~mm}$ in diameter in a $6 \mathrm{~mm}$ stainless steel die at 2 tons for 2 minutes. 
Figure 26 shows the standard addition curves and extrapolated concentration. All of the elements produced a curve with an $\mathrm{R}^{2}$ coefficient of 0.99 . This method was also very accurate and precise with the uncertainty in the calculated concentration and the \%bias less than $10 \%$ for all elements except Zn, which had a \%bias of $21 \%$.

\subsubsection{Conclusions}

Table 8 summarizes the results for the pelleting method with single point calibration, external calibration, and standard addition. The results for the pelleting methods were closer to the desired accuracy and precision values than the dried spot methods. Standard addition preformed the best in terms of accuracy with a \%bias less than $10 \%$ for all elements except $\mathrm{Zn}$. Matrix effects could have been an issue with the other calibration strategies since they relied on the use of a whey powder to determine the concentration in a milk sample. Based on the results, zinc was excluded from the element menu, because it repeatedly performed poorly. All methods were able to produce precise measurements. Since the standard addition method produced the most accurate results, this pelleting method was selected as the method to use for the rest of this study. 

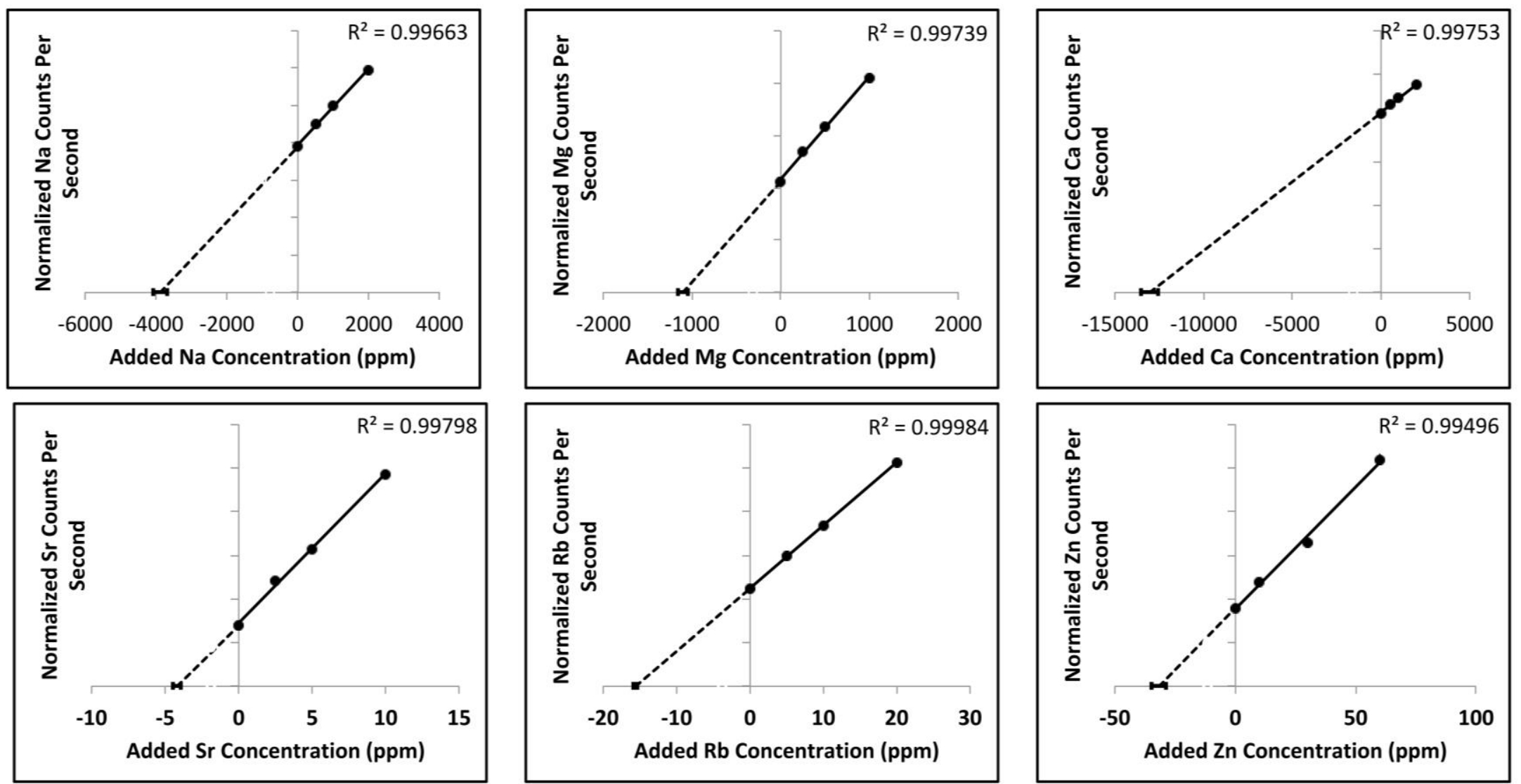

Figure 26 - Standard addition curves for $\mathrm{Na}, \mathrm{Mg}, \mathrm{Ca}, \mathrm{Sr}, \mathrm{Rb}$, and $\mathrm{Zn}$ in IAEA-153 using the pelleting method. The y error bars represent \pm 1 standard deviation. The $x$ error bars represent $\pm 1 S_{X E}$ 
Table 8 - Figures of merit for IAEA-153 using the two different pellet sample preparations and the different calibration strategies. The values in red are higher than the desired $\pm \mathbf{1 0 \%}$. The uncertainty for the standard addition and external calibration methods in the table are given as either $\mathbf{S}_{\mathrm{XE}}$ (\%RSD) or $\mathrm{S}_{\mathbf{x} 0}(\% \mathrm{RSD})$

\begin{tabular}{|c|c|c|c|c|c|c|c|c|c|c|}
\hline \multirow{3}{*}{\begin{tabular}{|l|} 
Method \\
Calibration
\end{tabular}} & \multirow[b]{3}{*}{$\begin{array}{l}\text { Recommended Value IAEA-153 } \\
\text { (95\% Confidence Interval) }\end{array}$} & \multicolumn{6}{|c|}{ Pelleting Method \#1 } & \multicolumn{3}{|c|}{ Pelleting Method \#2 } \\
\hline & & \multicolumn{2}{|c|}{ Standard Addition } & \multicolumn{4}{|c|}{ (GLITTER Method) } & \multicolumn{3}{|c|}{ External Calibration } \\
\hline & & $\begin{array}{c}\text { Conc. } \\
\left(\mathrm{X}_{\mathrm{E}}, \mathrm{ppm}\right)\end{array}$ & $\begin{array}{l}\text { Uncertainty } \\
\left(S_{X_{E}}, \mathrm{ppm}\right) \\
(\% \mathrm{RSD})\end{array}$ & $\begin{array}{l}\text { Bias } \\
(\%)\end{array}$ & $\begin{array}{l}\text { Conc. } \\
\text { (ppm) }\end{array}$ & $\begin{array}{l}\text { Uncertainty } \\
\text { (\%RSD) }\end{array}$ & $\begin{array}{l}\text { Bias } \\
(\%)\end{array}$ & $\begin{array}{c}\text { Conc. } \\
\left(\mathrm{X}_{0}, \mathrm{ppm}\right)\end{array}$ & $\begin{array}{c}\text { Uncertainty } \\
\left(S_{X_{0}}, \mathrm{ppm}\right) \\
(\% \mathrm{RSD})\end{array}$ & $\begin{array}{l}\text { Bias } \\
(\%)\end{array}$ \\
\hline $\mathrm{Ca}$ & $12870(12540-13170)$ & 12800 & $465(3.6)$ & -0.5 & 12000 & 10 & -8.8 & 12900 & $401(3.1)$ & 0.2 \\
\hline $\mathrm{Mg}$ & $1060(1000-1150)$ & 1100 & $66(6.0)$ & 3.7 & 1080 & 7.9 & 1.8 & 1030 & $10(0.1)$ & -2.5 \\
\hline $\mathrm{K}$ & $17620(16480-18760)$ & - & - & - & 14700 & 2.0 & -16.8 & 15100 & $656(1.0)$ & -14.0 \\
\hline $\mathrm{Rb}$ & $14.03(12.27-16.10)$ & 15.4 & $0.2(1.3)$ & 9.8 & 14.3 & 0.7 & 1.8 & 15.4 & $0.4(2.5)$ & 5.8 \\
\hline $\mathrm{Na}$ & $4180(3870-4440)$ & 3870 & $206(5.3)$ & -7.4 & 4120 & 2.0 & -1.4 & 4270 & $85(2.0)$ & 5.6 \\
\hline $\mathrm{Sr}$ & $4.09(3.49-4.73)$ & 4.18 & $0.28(6.7)$ & 2.2 & 5.10 & 1.0 & 25.7 & 5.51 & $0.07(1.3)$ & 33.8 \\
\hline $\mathrm{Zn}$ & $39.56(37.66-41.23)$ & 31.3 & $2.9(9.3)$ & 20.9 & 49 & 11.0 & 22.7 & - & - & - \\
\hline
\end{tabular}




\section{CHAPTER 4. Creation of a Database}

The LA-ICP-MS standard addition method using the pelleting sample preparation produced accurate $(<10 \%$ bias $)$ and precise $(<10 \% \mathrm{RSD})$ for the milk reference standard IAEA-153. However not all milk powder are the same consistency and texture. A primary reference method was used to evaluate the performance of the LA-ICP-MS method for a sample set of authentic and commercial milk powders.

$k_{0}$-INAA is a primary method for quantitative elemental analysis. It is often used to certify reference materials. It is used in this study to further assess the accuracy of the LA-ICP-MS method. Comparison of the LA-ICP-MS results for 13 powdered milk samples to $k_{0}$-INAA was done by comparing $95 \%$ confidence intervals for each technique. The element menu was limited to $\mathrm{Na}, \mathrm{Ca}, \mathrm{Rb}$, and $\mathrm{Sr}$ for the comparison. $\mathrm{Zn}$ was excluded because it fell outside the $95 \%$ confidence interval for IAEA-153 using the standard addition method. $\mathrm{Mg}$ was not measured by $k_{0}$-INAA because it is a short-lived radionuclide. There were some samples that had a Sr concentration below the limit of detection for $k_{0}$-INAA. These samples are marked with <LOD. A better LOD for Sr was achieved with LA-ICP-MS than $k_{0}$-INAA (1 ppm vs. 5 ppm).

For LA-ICP-MS measurements, confidence intervals were calculated using:

$$
X_{E} \pm t_{(n-2)} S_{X_{E}}
$$

For this calculation, $\mathrm{n}$ is the number of calibration standards, 4 , and $\mathrm{t}$ is the Student's t-distribution. The $95 \%$ confidence interval for $k_{0}$-INAA was calculated by multiplying the combined standard uncertainty by 2. All intervals for LA-ICP-MS overlapped with those for reported for $k_{0}$-INAA. The intervals for $\mathrm{Ca}$ and $\mathrm{Na}$ are large for LA-ICP-MS because there were only 4 calibration standards used for the standard 
addition. Increasing the number of standards, $\mathrm{n}$, would decrease the $95 \%$ confidence interval.

The comparison of LA-ICP-MS to $k_{0}$-INAA further showed that the developed LA-ICP-MS method is capable of producing results similar to a primary reference method for a variety of powdered milk samples. The LA-ICP-MS data for the authentic samples (USA, Argentina, Slovenia) analyzed in this comparison was used to start the creation of a milk database. 
Table 9 - Comparison of the LA-ICP-MS method to k0-INAA. Reported are the $95 \%$ confidence intervals for both techniques

\begin{tabular}{|c|c|c|c|c|c|c|c|c|}
\hline \multirow[t]{2}{*}{ Sample } & \multicolumn{4}{|c|}{ LA-ICP-MS } & \multicolumn{4}{|c|}{$k_{0}$-INAA } \\
\hline & $\mathrm{Na}$ & $\mathrm{Ca}$ & $\mathrm{Rb}$ & $\mathrm{Sr}$ & $\mathrm{Na}$ & $\mathrm{Ca}$ & $\mathrm{Rb}$ & $\mathrm{Sr}$ \\
\hline \multirow{2}{*}{ USA 1} & 3750 & 12800 & 15.4 & 4.21 & 4310 & 12700 & 17.4 & \multirow{2}{*}{$<\mathrm{LOD}$} \\
\hline & \pm 694 & \pm 2030 & \pm 1.3 & \pm 1.39 & \pm 302 & \pm 904 & \pm 1.2 & \\
\hline \multirow{2}{*}{ USA 2} & 3960 & 11500 & 16.7 & 7.59 & 4060 & 10900 & 17.0 & 8.62 \\
\hline & \pm 134 & \pm 1280 & \pm 2.5 & \pm 0.40 & \pm 284 & \pm 786 & \pm 1.2 & \pm 1.70 \\
\hline \multirow{2}{*}{ USA 3} & 4050 & 11800 & 15.6 & 7.83 & 3970 & 11100 & 16.5 & 7.10 \\
\hline & \pm 137 & \pm 1420 & \pm 0.4 & \pm 0.89 & \pm 278 & \pm 800 & \pm 1.2 & \pm 1.38 \\
\hline \multirow{2}{*}{ ARGENTINA 1} & 4300 & 11500 & 17.5 & 6.87 & 4330 & 10900 & 17.3 & 7.11 \\
\hline & \pm 1705 & \pm 5680 & \pm 8.6 & \pm 0.24 & \pm 304 & \pm 800 & \pm 1.2 & \pm 2.24 \\
\hline \multirow{2}{*}{ ARGENTINA 2} & 2650 & 7850 & 4.78 & 16.9 & 2770 & 8890 & 5.00 & 17.4 \\
\hline & \pm 98 & \pm 1410 & \pm 1.54 & \pm 9.2 & \pm 196 & \pm 902 & \pm 0.46 & \pm 4.8 \\
\hline \multirow{2}{*}{ SLOVENIA 1} & 3480 & 7640 & 4.20 & 19.5 & 3700 & 9060 & 3.97 & 27.4 \\
\hline & \pm 150 & \pm 427 & \pm 1.30 & \pm 3.5 & \pm 264 & \pm 974 & \pm 0.40 & \pm 5.6 \\
\hline \multirow[b]{2}{*}{ SLOVENIA 2} & 3730 & 12800 & 16.5 & 3.05 & 4110 & 11800 & 17.9 & \multirow[b]{2}{*}{$<$ LOD } \\
\hline & \pm 300 & \pm 4810 & \pm 1.7 & \pm 0.93 & \pm 288 & \pm 884 & \pm 1.2 & \\
\hline \multirow{2}{*}{ SLOVENIA 3} & 4020 & 13500 & 16.4 & 3.45 & 4150 & 12000 & 17.4 & \multirow{2}{*}{$<\mathrm{LOD}$} \\
\hline & \pm 624 & \pm 3080 & \pm 1.0 & \pm 1.81 & \pm 292 & \pm 914 & \pm 1.2 & \\
\hline \multirow{2}{*}{ COMMERCIAL 1} & 3870 & 13700 & 13.4 & 2.35 & 4040 & 12900 & 14.7 & \multirow{2}{*}{$<$ LOD } \\
\hline & \pm 567 & \pm 7300 & \pm 1.3 & \pm 1.82 & \pm 292 & \pm 962 & \pm 1.0 & \\
\hline \multirow{2}{*}{ COMMERCIAL 2} & 3680 & 11100 & 11.1 & 5.10 & 3650 & 10800 & 11.3 & 5.37 \\
\hline & \pm 122 & \pm 561 & \pm 0.8 & \pm 0.54 & \pm 256 & \pm 774 & \pm 0.8 & \pm 1.58 \\
\hline \multirow{2}{*}{ COMMERCIAL 3} & 3740 & 11000 & 24.7 & 7.98 & 3960 & 11300 & 25.9 & 10.7 \\
\hline & \pm 179 & \pm 1020 & \pm 1.7 & \pm 1.06 & \pm 280 & \pm 862 & \pm 1.8 & \pm 2.4 \\
\hline \multirow[b]{2}{*}{ COMMERCIAL 4} & 2870 & 8400 & 9.40 & 3.60 & 2980 & 8500 & 9.71 & \multirow[b]{2}{*}{$<$ LOD } \\
\hline & \pm 9 & \pm 1580 & \pm 0.40 & \pm 0.51 & \pm 208 & \pm 666 & \pm 0.70 & \\
\hline \multirow{2}{*}{ COMMERCIAL 5} & 2700 & 8630 & 26.5 & 3.99 & 2690 & 8050 & 28.5 & \multirow{2}{*}{$<$ LOD } \\
\hline & \pm 140 & \pm 986 & \pm 0.4 & \pm 0.63 & \pm 188 & \pm 640 & \pm 2.0 & \\
\hline
\end{tabular}




\subsection{Collection of Data for Authentic Samples}

Since the collection of authentic samples was a difficult task, the participants were asked if they would be willing to share their data for the creation of a database. Those that were willing to share elemental data were asked to submit their results for the IAEA-153 reference standard to ensure that the lab's method is accurate and precise.

\subsubsection{Inter-Comparison}

Research laboratories participating in the IAEA CRP were asked to analyze IAEA-153 for $\mathrm{Na}, \mathrm{Mg}, \mathrm{Ca}, \mathrm{Zn}, \mathrm{Rb}$, and $\mathrm{Sr}$ using their own methods, in order to evaluate their performance. The techniques used included LA-ICP-MS, solution ICP-MS, and ICP-OES. Although there are some differences among labs, each lab used a standard operating procedure that has previously been optimized.

Figure 27 shows the data from five participating labs that provided accurate results for the elements of interest and how it compares to the reported value for IAEA153 and $k_{0}$-INAA. The standard deviation lines were calculated from the IAEA-153

reference sheet. For most labs, the concentration of the element fell within \pm 3 standard deviations. One element, $\mathrm{Zn}$, fell outside \pm 3 standard deviations for labs $\mathrm{A}$ and $\mathrm{B}$ (not shown on Figure 27). For this reason, $\mathrm{Zn}$ was excluded from the element menu for discrimination. Also Labs A, B, and D produced inaccurate or had a high standard deviation for Ca. This element was also excluded from the element menu. Lab A used LA-ICP-MS. Labs B, D, E used solution ICP-MS. Lab C used ICP-OES for Na, Mg, Ca, $\mathrm{Zn}$ and ICP-MS for $\mathrm{Rb}$ and $\mathrm{Sr}$. There were three other laboratories that submitted data, 
however, their methods were not accurate for most of the elements of interest and are not shown here.

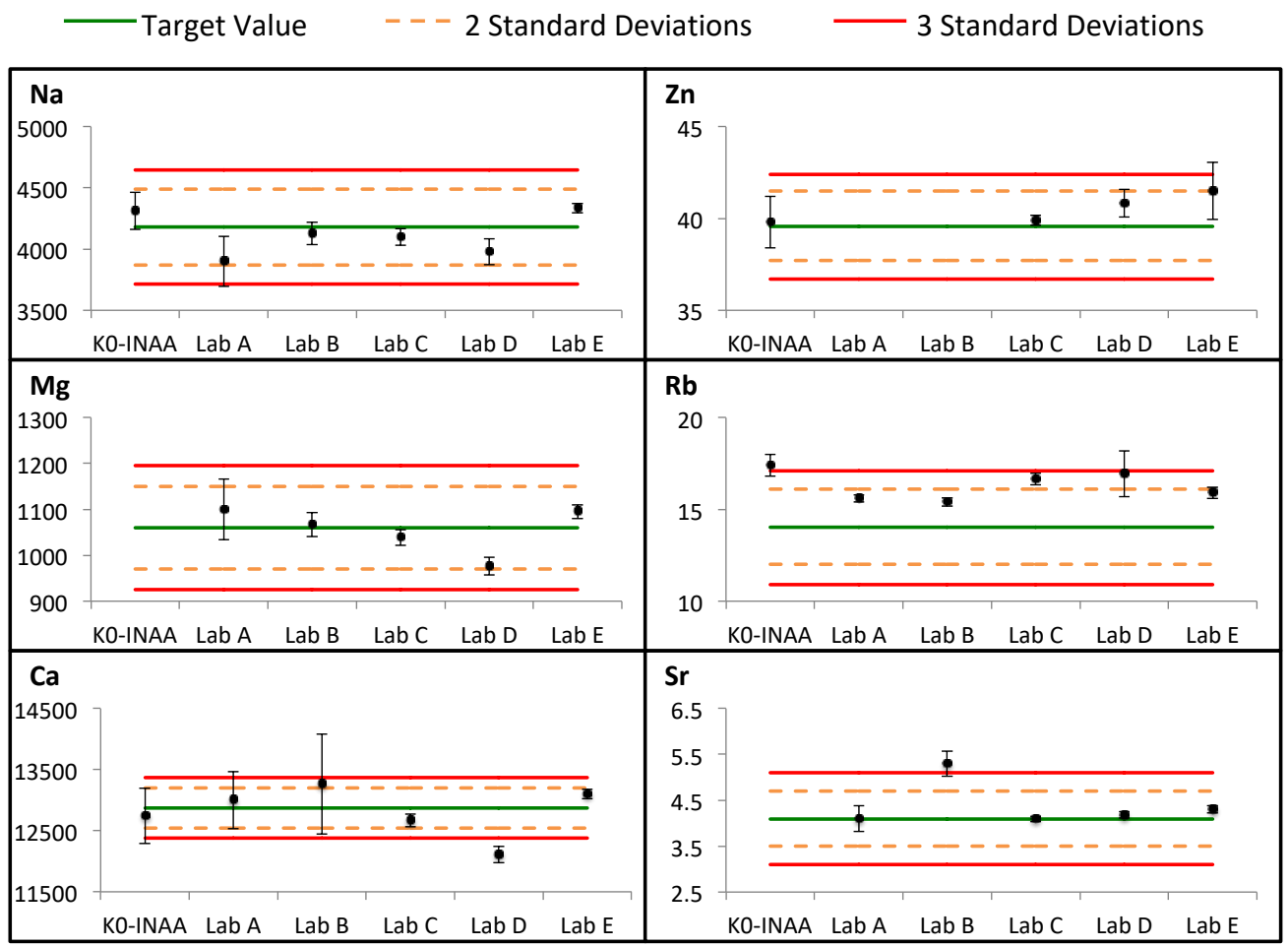

Figure 27 - Inter-laboratory comparison of select elements in IAEA-153. Shown are the mean values determined by each lab for each element. Labs $A$ and B produced results for $\mathrm{Zn}$ that fell outside \pm 3 standard deviations. Labs A, B, and D produced either inaccurate results or had high standard deviations for Ca. Both $\mathrm{Zn}$ and $\mathrm{Ca}$ were removed from the element menu.

\subsubsection{Preliminary Provenancing Study}

Each lab shown in the inter-comparison studies collected authentic milk samples, analyzed them using their own method, and submitted the data. Countries represented include: Argentina, Russia, Singapore, Slovenia, and the United States. For some countries, the data submitted represents a small area and not the entire country. For example, the United States data represents three samples from a milk shed. The Slovenia data represents two drying facilities, two samples from each. The data from Singapore represents two farms, five samples from each. The data from Argentina are from one to 
three farms within each of four distinct regions. The data spread from Russia is for 41 samples collected from farms located throughout the southern half of the entire country $(\sim 9000 \mathrm{~km})$.

Principle component analysis (PCA) was used to evaluate the data from Argentina, Russia, Singapore, Slovenia, and the United States. To generate the PCA plots the following elements/ratios were used: $\mathrm{Mg}, \mathrm{Rb}, \mathrm{Sr}, \mathrm{Na} / \mathrm{Sr}, \mathrm{Mg} / \mathrm{Sr}$. These were selected, on the basis of their discriminating capability, using stepwise linear discriminant analysis in JMP.

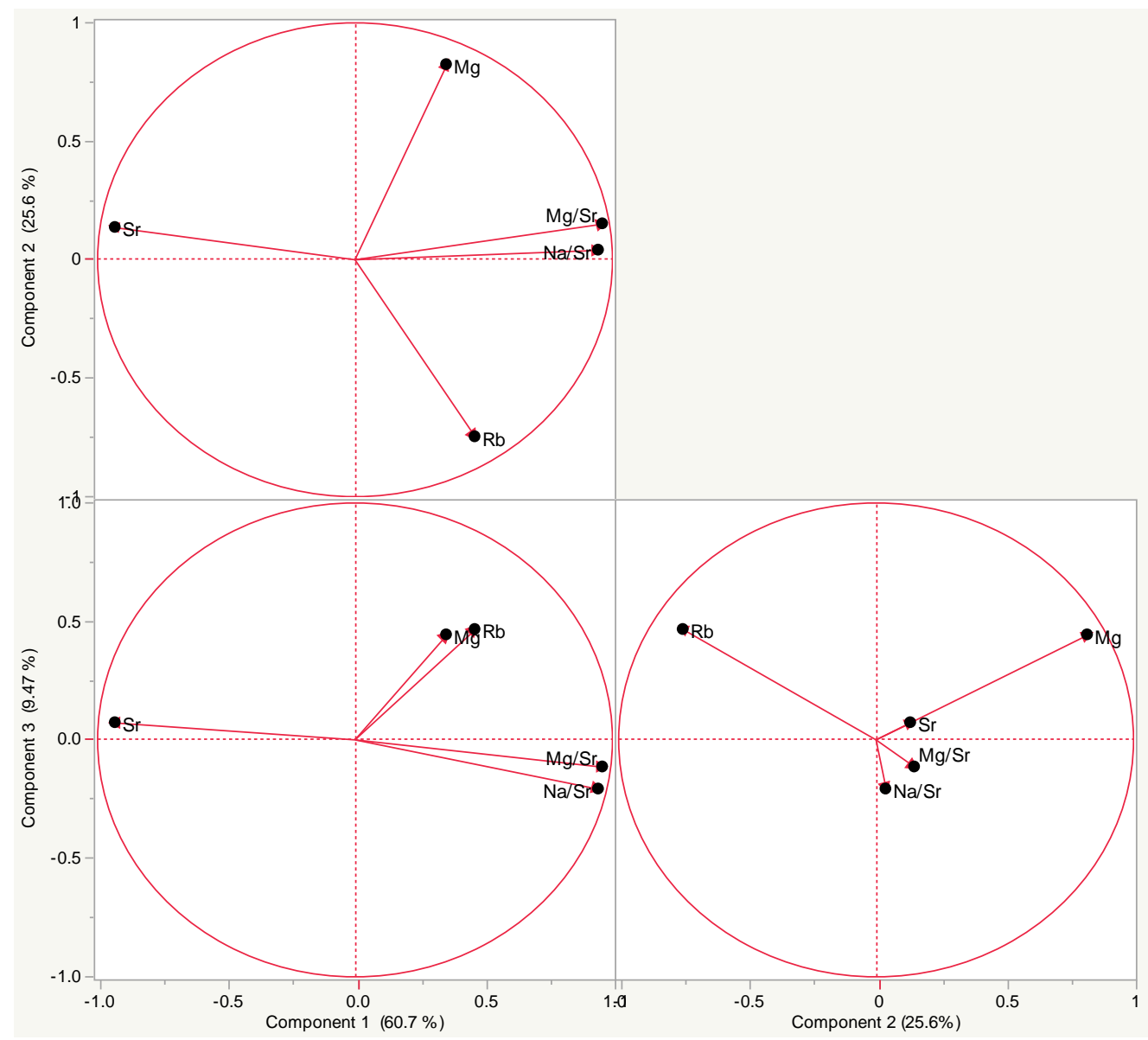

Figure 28 - The loading plots used for the PCAs 
Figure 28, depicts the loading plots for the principle component. Component 1 accounts for $60.7 \%$ of the variation and is most influenced by $\mathrm{Sr}, \mathrm{Mg} / \mathrm{Sr}$, and $\mathrm{Na} / \mathrm{Sr}$. Component 2 accounts for $25.6 \%$ and is most influenced by $\mathrm{Mg}$ and $\mathrm{Rb}$. Component 3 accounts for $9.47 \%$ of the variation and is most influenced by $\mathrm{Rb}, \mathrm{Mg}, \mathrm{Sr}, \mathrm{Na} / \mathrm{Sr}$, and $\mathrm{Mg} / \mathrm{Sr}$. Figure 29 shows the scatterplots generated using the principle components. The black Xs in the plot represent the data for IAEA-153 submitted by each country. The IAEA-153 samples are grouped together further showing that each country was able to detect similar concentrations using their methods. The samples from Russia were not distinguished from those from Argentina, the United States, and Slovenia, Figure 29. This inability to distinguish the samples is mostly a result of the expected large elemental variation observed within the many Russian samples due to the different geological sources, in comparison to the limited samples (and geographic representation) from Argentina, the United States, and Slovenia. Because the high variation in the Russian samples, the PCA was redone excluding Russia to better evaluate the grouping between the remaining countries, Figure 30. IAEA-153 was included in the PCA plots to further show that the IAEA-153 data clusters together even though it was analyzed by different methods in different labs. 


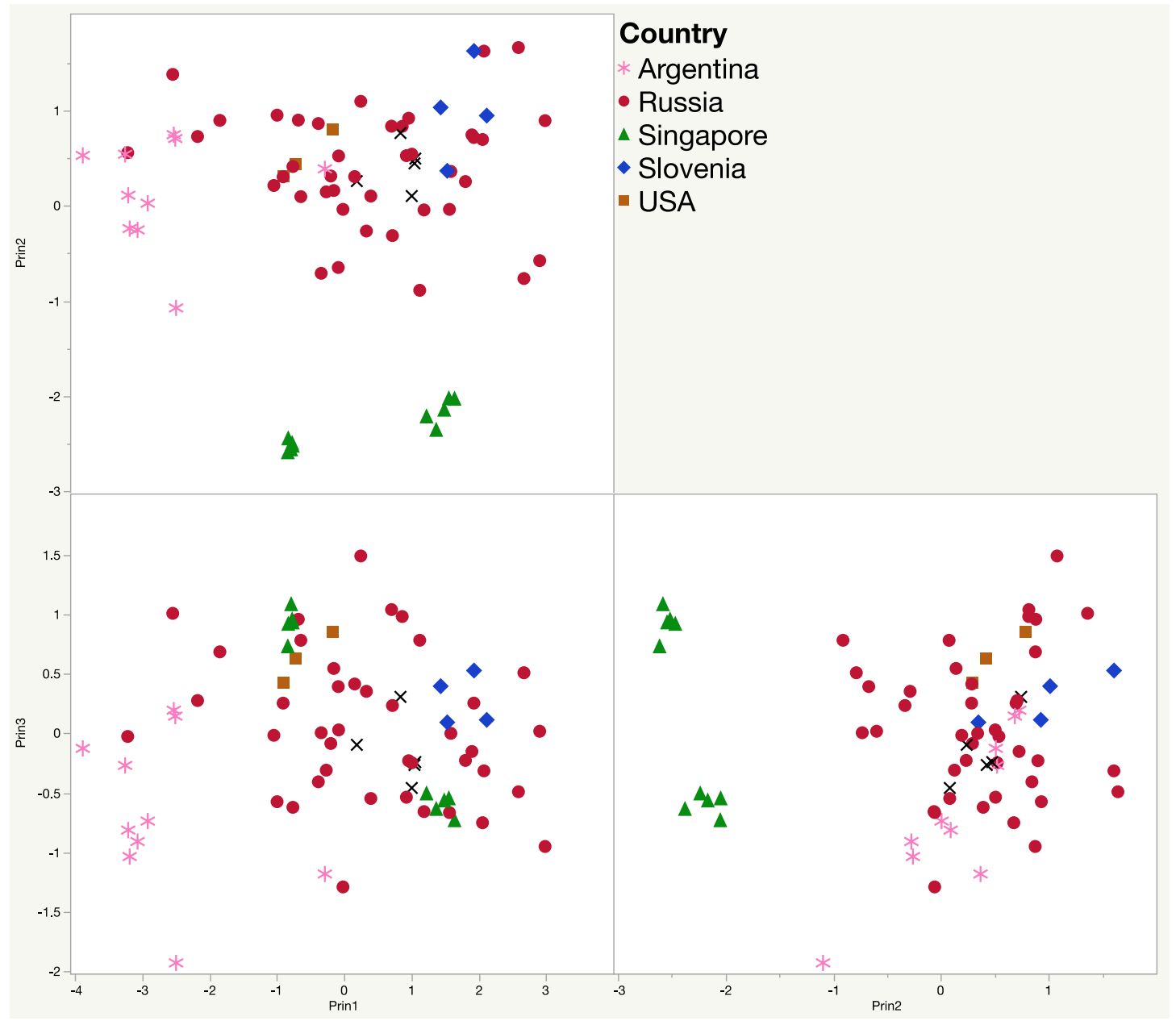

Figure 29 - A PCA plot depicting the authentic samples from each country. The pink stars represent the data from Argentina. The red circles represent the data from Russia. The green triangles represent the data from Singapore. The blue diamonds represent the data from Slovenia. The orange squares represent the data from the USA. The black Xs represent the data submitted from each lab for IAEA-153 


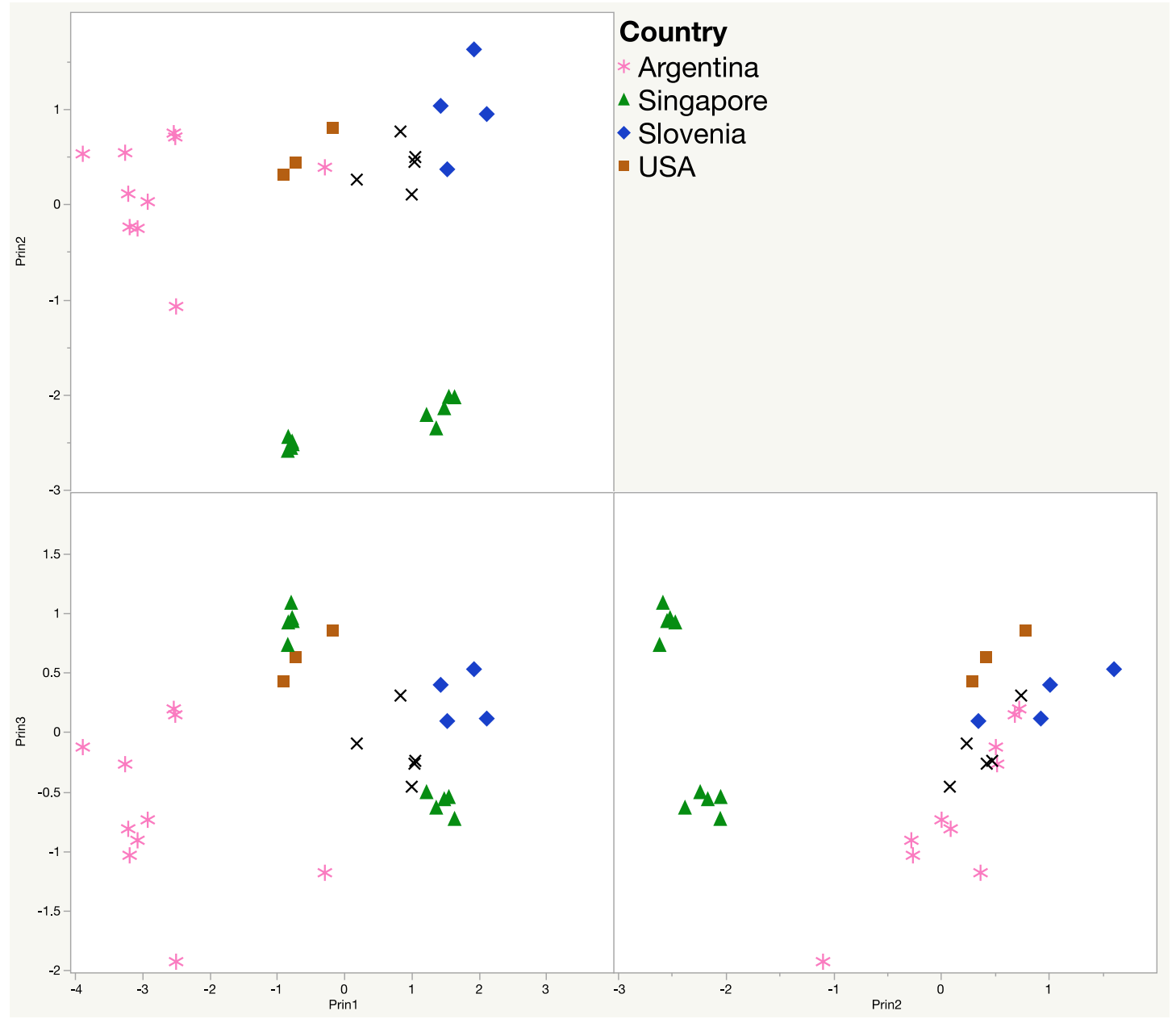

Figure 30 - A PCA plot without Russia. The pink stars represent the data from Argentina. The green triangles represent the data from Singapore. The blue diamonds represent the data from Slovenia. The orange squares represent the data from the USA. The black Xs represent the data submitted from each lab for IAEA-153

Looking at principle component 1 in Figure 30, it can be discovered that the USA and part of the Singapore samples have a positive value while the other countries, Slovenia and Argentina, have a negative value. Interestingly the data from the two farms from Singapore clusters into two separate groups with each group representing a single farm. One of the farms has a negative value for principle component 1 while the other is positive. The combination of variables responsible for the separation using principle component 1 are $\mathrm{Sr}, \mathrm{Mg} / \mathrm{Sr}$ and $\mathrm{Na} / \mathrm{Sr}$. Using principle component 1, the samples from 
Argentina are separated from the other countries. The two farms from Singapore are separated from the other countries by principle component 2 in which they have a negative value of around -2 and the other countries have a positive value or a value close to -1 . The combination of variables responsible for the separation using principle component 2 are $\mathrm{Mg}$ and $\mathrm{Rb}$. The two Singapore farms are also separated from each other using principle component 3. The separation using principle component 3 is most influenced by $\mathrm{Mg}, \mathrm{Rb}, \mathrm{Mg} / \mathrm{Sr}$, and $\mathrm{Na} / \mathrm{Sr}$. 


\section{CHAPTER 5. Analysis of Milk Powder Conclusion}

Since milk is commonly imported and exported as a powder, the main focus of this study was the development of a solid sampling method for the elemental analysis of powdered milk. This study tested a variety of sample preparations such as tape mounting, dried discs, dried spots, and pellets. This study also tested single point calibration, external calibration curves, and standard addition curves. Each sample preparation and calibration combination was analyzed using LA-ICP-MS. The accuracy and precision of each sample preparation method and calibration strategy was evaluated using IAEA-153. Though the tape mounting, dried discs, and dried spot sample preparations were quick and easy, the results in terms of percent accuracy and precision did not meet the desired values. The dried spot method was one of the more accurate methods, however, the \%bias was higher than the desired $10 \%$ for both external calibration curves and standard addition curves. The addition of ICP-MS element standards to milk powder also proved challenging. Since the ICP-MS standards are usually in 2-3\% nitric acid, addition of too large a spike would cause precipitation of the milk proteins and partially digestion of the sample. For the creation of the standard addition curves using the pelleting sample preparation, care was taken to avoid large volumes of element standards being added to a single calibration pellet. The calibration pellets were setup so each one received $\sim 175 \mathrm{uL}$ of the ICP-MS element standards. This standard addition pelleting method proved to be the most accurate (<10\% bias) and precise ( $<10 \%$ RSD).

Since milk powder can come in various consistencies, the results for 13 milk powder samples from both authentic and commercial sources analyzed by the developed LA-ICP-MS method were compared to a primary method, $k_{0}$-INAA. The $95 \%$ confidence 
intervals for the LA-ICP-MS results overlapped with the $95 \%$ confidence intervals of the primary method further showing that the developed LA-ICP-MS method can be used to analyze a variety of milk powder samples.

The LA-ICP-MS standard addition method yields accurate elemental concentrations for $\mathrm{Na}, \mathrm{Mg}, \mathrm{Ca}, \mathrm{Rb}$, and $\mathrm{Sr}$ without the need for acid digestion. Though LA-ICP-MS is an expensive technique, the analytical approach developed in this study may also be used for other laser-based methods such as LIBS, which would be a more convenient, faster, and economical tool than LA-ICP-MS that would especially be useful for developing countries.

To test the discriminating potential of this method, data from authentic milk powder samples from five countries were collected and evaluated. The data submitted were limited to the following elements: $\mathrm{Na}, \mathrm{Mg}, \mathrm{Rb}$, and $\mathrm{Sr}$. The limited element menu was able to differentiate the samples from Singapore, Argentina, the United States, and Slovenia, from each other. However, the collected data represented a few localized areas and does not account for the total variation within every country. Samples collected from a large geographic area within Russia produces an expected large variation in the elemental profiles and therefore the limited element menu was not able to distinguish the Russian milk samples from those for Argentina, the United States, and Slovenia. The goal of being able to determine if a commercial milk originated from the country on the label has not been met. However, the few samples from Singapore appear to be very different from the samples from the drying facilities in the United States and Slovenia, and from the samples from Russia. If milk produced in Singapore was labeled as being made in the Russia, the elemental profile of that milk would not line up with that the current profile 
found in southern Russia. The data from the participating laboratories suggest that database creation is a viable option and the addition of authentic samples from interested countries and geographic areas may provide a tool to detect fraudulently labeled commercial milk.

My recommendations for future studies are the collection of additional samples that would need be analyzed for each county to better account for the within country variation. The dried spot method with the ICP-MS solution external calibration does show some promise as a quick sample preparation method. However, it appears to be suffering from a systematic bias. More research should be done to determine what is causing the high bias and if this is a viable option. Also, the addition of isotope ratios, additional trace elements, or another discriminating factor may help in further distinguishing one country from another thus making it easier to determine if an unknown milk could have originated from a specific country. 


\section{CHAPTER 6. Introduction to Forensic Analysis of Glass}

\subsection{Research Motivation}

Glass fragments are encountered as forensic evidence from broken windows in burglaries, from automobile hit-and-run accidents, and from other crimes. Forensic glass examiners are asked to analyze and compare the physicochemical properties of glass collected from a known source (Ks) to fragments that have been recovered from an unknown or questioned source (Qs). In most forensic labs today, the glass Ks and Qs are compared using refractive index measurements and trace elemental concentrations in order to determine whether the Q glass samples could have originated from the same K source of broken glass. Laser Ablation-Inductively Coupled Plasma-Mass Spectrometry (LA-ICP-MS) has been referred to as the "gold standard" for the elemental analysis of glass and many researchers have reported excellent analytical figures of merit for the measurement as well as a fit-for-purpose utility for the forensic comparison [42-47]. However, when it comes to reporting the results of the casework, the strength of the evidence is usually not conveyed.

While the forensic community has reached consensus on the analytical protocol for the use of LA-ICP-MS and recommended a comparison criterion with known error rates for a limited number of scenarios, it has not yet reached consensus on how to interpret the weight of a glass comparison for reporting to the court in verbal terms. Some analysts simply state that the $K$ and $Q$ could have originated from the same broken glass pane or another source produced with the same physical and chemical characteristics. Others provide more details and state the discrimination limitations of LA-ICP-MS: the $K$ 
and $Q$ could have originated from the same broken glass pane or another source produced at the same manufacturing plant around the same time. One approach that is gaining support is the use of a verbal scale that is divided by the presence or absence of individual or class characteristics as well as the discrimination potential of the techniques used [48]. This approach may be considered as subjective since it relies on the analyst's personal experience and assessment of the glass evidence. More objective methods include the application of statistical tools to calculate the random match probability that glass samples from different sources are found to share the same trace elemental profile (reported to be $\sim 0.1 \%$ ) $[45,46]$ or a frequency of a specific elemental profile. In order to calculate these, however, a suitable database of trace elemental data from glass samples is required.

\subsection{Significance of Study}

The main significance of this study is the creation of a new database for the evaluation of different statistical approaches to aid in the interpretation of glass evidence. This research is broken down into four main parts: the collection of existing elemental glass databases and the creation of a new vehicle database, the evaluation of current ASTM guidelines for the analysis and comparison of glass evidence, and the assessment of different statistical models for the objective and quantitative interpretation of glass evidence using a large user community of glass examiners named the Glass Interpretation Working Group (GIWG).

First, forensic glass examiners were surveyed to determine if they had an

elemental glass database and if it is currently being used to aid in the interpretation of 
glass evidence. Specifics such as number of samples, type of glass, years represented, element menu etc. were collected for each database. In addition to the collection of existing databases, a new vehicle glass database was also created from the LA-ICP-MS analysis of 420 glass samples from 210 different vehicles representing 26 different vehicle manufacturers and manufacturing dates ranging from 2004 to 2017. Each glass database is a snapshot of a specific time and location. Therefore the interpretation of glass evidence may change depending on what database was used. This possibility was investigated in this research. It may also be possible to combine the existing databases even though instrumentation differs between labs. This possibility was evaluated through the use of inter-laboratory studies. By comparing each lab's elemental data for specific glass samples and a control glass, similarities and differences could be detected for each element and used to determine if databases from the labs could be combined.

For the inter-laboratory studies, samples from the new vehicle database were sent to LA-ICP-MS users in the form of a mock case. Participants were asked to analyze the glass samples, compare the Ks and Qs, and report their findings. The data they submitted was used to evaluate the ASTM E2927match criterion and also used to assess different statistical approaches to interpretation.

This research provided forensic experts with a new look at evidence interpretation. Though no consensus was reached, the participants realized the need for a better way to interpret glass evidence and are willing to consider alternative approaches. Additionally, this research brought together glass databases that can be studied further to obtain a better grasp on the elemental profile of glass and how it differs throughout time and location, and can also be subjected to other statistical tests. 


\subsection{Composition of Glass}

Glass is located all around us and is available in an assortment of shapes and compositions. The raw materials and the production process create variations between glass batches between manufacturers and even within the same manufacturer that can be detected in glass when sensitive elemental techniques such as ICP-MS are used.

Glass is defined as " an inorganic production of fusion that has been cooled to a rigid condition without crystallization" [49]. Like the definition suggests, glass is made by melting inorganic materials, usually oxide minerals together, but the chemicals added and how the glass is formed depends on the intended end use. This section will explain some of the main components of glass and then describe the types of glass most encountered in casework.

The components of glass are classified according to their function: formers, fluxes, modifiers, stabilizers etc. The formers generally sculpt the framework of the glass structure. These are compounds such as silica $\left(\mathrm{SiO}_{2}\right)$ sand. Silica requires high temperatures to undergo fusion, but chemicals such as sodium carbonate $\left(\mathrm{Na}_{2} \mathrm{CO}_{3}\right)$ and potassium oxide $\left(\mathrm{K}_{2} \mathrm{O}\right)$ can be added to reduce the temperature needed. These chemicals are called fluxing agents. To increase the durability of the glass limestone $(\mathrm{CaO})$ and/or alumina $\left(\mathrm{Al}_{2} \mathrm{O}_{3}\right)$ are added, stabilizers. Other additives give glass specific properties such as color (Table 10), heat resistance, or the ability to absorb radiation [49].

For some products, manufacturers want the end results to be clear and colorless. However, one of the main ingredients, sand, often contains trace amounts of iron that cause a green color. To offset the green color and create an end product that appears colorless, manufacturers will often add other colorants such as selenium and cobalt. Table 
10 shows a list of colorants that can be added to glass to either add color or offset a color caused by contaminants [49].

Table 10 - Displayed are common coloring elements and the color each produces [49]

\begin{tabular}{|ll|}
\hline Colorant & Color \\
\hline Mron & Green, brown, clue \\
Chromium & Purple \\
Vanadium & Green, yellow, pink \\
Copper & Green, blue, grey \\
Cobalt & Blue, green, red \\
Nickel & Blue, green, pink \\
Uranium & Yellow, purple \\
Titanium & Yellow, brown, green \\
Neodymium & Purple, brown \\
Praseodymium & Purple \\
Cerium & Green \\
Carbon and sulphur & Amber, brown \\
Cadmium sulphide & Yellow \\
Antimony sulphide & Red \\
Selenium & Pink, red \\
Gold & Red \\
\hline
\end{tabular}

Glass is often categorized by its major ingredients. For example, one type of glass that will be referred to heavily throughout this research is soda-lime glass. The main additives of this glass are sodium carbonate $\left(\mathrm{Na}_{2} \mathrm{CO}_{3}\right)$ and limestone $(\mathrm{CaO})$. This type of glass is used in bottles, architectural windows, and vehicle windows. Two other types of glass worth mentioning are lead glass and borosilicate glass. Leaded glasses are created 
by the addition of lead oxide $(\mathrm{PbO})$. These glasses are used for stemware, electrical connections, and eyeglasses. Borosilicate glasses are created by the addition of boric oxide $\left(\mathrm{B}_{2} \mathrm{O}_{3}\right)$. These glasses are temperature and thermal shock resistant and used as cookware and automobile headlamps [49]. The range of composition varies between and within each glass types, but Table 11 lists some typical compositions. The inorganic elements in the final composition of the glass are present at different concentrations ranging from percent levels to low ppm levels.

Table 11 - Glass compositions with values expressed as weight percent [49]

\begin{tabular}{|lcccccccccc|}
\hline Glass Type & $\mathrm{SiO}_{2}$ & $\mathrm{Al}_{2} \mathrm{O}_{3}$ & $\mathrm{Fe}_{2} \mathrm{O}_{3}$ & $\mathrm{CaO}$ & $\mathrm{MgO}$ & $\mathrm{Na}_{2} \mathrm{O}$ & $\mathrm{K}_{2} \mathrm{O}$ & $\mathrm{Cr}_{2} \mathrm{O}_{3}$ & $\mathrm{PbO}$ & $\mathrm{B}_{2} \mathrm{O}_{3}$ \\
\hline Container: Flint & 72.6 & 1.6 & 0.05 & 11.0 & 0.1 & 13.7 & 0.5 & - & - & - \\
Container: Amber & 72.7 & 1.9 & 0.22 & 10.0 & - & 13.8 & 1.0 & - & - & - \\
Container: Green & 72.0 & 1.1 & 0.1 & 8.2 & 2.1 & 15.1 & 1.0 & 0.19 & - & - \\
Float: (Soda-lime) & 72.8 & 1.4 & 0.1 & 8.2 & 3.8 & 12.8 & 0.8 & - & - & - \\
Borosilicate & 80.2 & 2.6 & 0.07 & 0.1 & - & 4.5 & 0.3 & - & - & 12.3 \\
Lead Crystal & 54.9 & 0.1 & 0.02 & - & - & 0.2 & 12.3 & - & 31.9 & 0.5 \\
Optical Glass & 48.0 & 0.2 & - & 0.3 & - & 5.2 & 1.2 & - & 45.1 & - \\
\hline
\end{tabular}

The use of recycled glass or cullet is commonly employed in the manufacture of glass to reduce the cost of the manufacturing process. Factory created waste is recycled to the furnace and added to the new batch of glass, which typically adds more variability and heterogeneity between batches of glass originating from the same plant.

Just as there are many different glass compositions, there is also an assortment of ways to manufacture glass depending on the product end use. The following sections describe the main manufacturing processes of glass typically encountered in forensic examinations, containers and flat glass. 


\subsection{Manufacturing of Containers and Flat Glass}

As previously stated, glass is composed of several inorganic compounds which are added based on the desired properties of the end product. Container glass and float glass are both examples of soda-lime glass. Once the raw materials are mixed, the components are melted together in a furnace. The molten glass is refined and homogenized by a combination of thermal and mechanical stirring. During the next stage the glass is formed into containers or a flat sheet.

\subsubsection{Container Glass}

Containers are made from globs of molten glass. For glass bottles, a hollow iron pipe is inserted into the glob and air is used to blow the glass. While still hot, the glass is inserted into a mold and the blowing process repeats. The molds are then passed through kilns, which allow the glass to slowly cool.

\subsubsection{Flat Glass}

Flat glass is made by one of two processes, either the float glass process or rolling. The float glass process is used for the manufacture of architectural and vehicle glass and will be the main focus of this section. The rolling process is used for wired and patterned glass.

Molten glass flows into the float chamber containing molten tin. As the molten glass floats on the tin, it forms a ribbon that is drawn along the tin bath to the desired thickness. As the glass flows, the ribbon is cooled until it can be safely rolled to the annealing kiln without marking the surfaces. Once annealed, the glass is cut into the desired dimensions. 


\subsubsection{Finishing Touches on Glass}

After the containers and/or flat sheets have been manufactured, additional steps can be taken to alter the properties and form the final product. Tempering of the glass is a step involved in the manufacture of side and rear vehicle windows. This process

strengthens the glass and also causes the glass to shatter into small pieces after a collision. Tempering is accomplished by first heating the glass up and then rapidly cooling it. The surface of the glass will cool faster than the center. As the center cools, it contracts pulling and thus compressing the rigid surfaces. As the outer surfaces compress, tension buildups in the center.

Laminating the glass is another technique used for vehicle glass. During this process, two or panes of float glass are bonded together by a thin plastic film. In the United States, all front windshields must be laminated glass.

When it comes to container glass, coatings are often added for decoration or to strengthen and protect the glass. There are two processes used to add coatings, hot end coating and cold end coating. Hot end coatings are added after the glass leaves the forming tank and before it is annealed. These coatings are sprayed onto the molten glass and are usually either compounds containing tin or titanium. Cold end coatings are added while the glass is being annealed. These coating are also sprayed, but are commonly organic compounds such as organic waxes and polyethylene glycols.

\subsection{Thickness and Refractive Index}

Forensic glass examiners are asked to analyze and compare the physicochemical properties of glass collected from a known source (Ks) to fragments that may have been 
recovered from an unknown or questioned source (Qs). The glass Ks and Qs are compared using physical characteristics such as color and thickness, refractive index measurements, and/or trace elemental concentrations in order to determine whether the $\mathrm{Q}$ glass samples could have originated from the same K source of broken glass.

The simplest techniques include measuring the thickness of glass samples and looking at the color. This is only applicable in cases where both the K and Q are full thickness fragments. Various studies have shown that the thickness of float glass does not differ much within a float ribbon. The variation was found to be around $0.15 \mathrm{~mm}[50,51]$. For this reason, when forensic glass examiners compare thickness measurements for a $\mathrm{K}$ and $\mathrm{Q}$, most create a comparison interval using the mean of the $\mathrm{K} \pm 0.15 \mathrm{~mm}$ and compare the thickness of the Q fragment. If the Q thickness falls within the comparison interval, the samples are indistinguishable.

The next technique that has been shown to add some discrimination is refractive index (RI). Refractive index is measure of how much lights bend after entering a medium. It is also defined as the ratio of the velocity of light in a vacuum to its velocity in a specified medium. Refractive index became useful tool for forensic scientists. The method used mostly today to measure the RI of glass is the oil immersion method with a phase contrast microscope. The method works on the principle that the refractive index of a liquid will change as the liquid is heated. The refractive index of glass is determined by placing the glass fragment in silicone oil and heating/cooling the oil until the glass fragment appears invisible. At this point, the oil and glass will have the same refractive index. A calibration curve is created with glasses of known RI plotted vs. the temperature of the oil at which point each glass disappeared. The RI of an unknown glass sample is 
then determined using $\mathrm{RI}=\mathrm{a}+\mathrm{bT}$ where $\mathrm{a}$ is intercept at $0{ }^{\circ} \mathrm{C}, \mathrm{b}$ is the slope of the calibration curve, and $\mathrm{T}$ is the temperature of the oil at which point the unknown glass disappeared. A GRIM II, glass refractive index measurement, is employed for these measurements. The GRIM II is equipped with a phase contrast microscope with a fixed illumination wavelength that is also equipped with a video camera, a hot stage, and electronic temperature controller [52]. Refractive index has shown to decrease in discrimination potential with the technological advances and standardizations in glass manufacturing; however, it is still used by some forensic laboratories and can provide some additional information about a $\mathrm{K}$ and Q sample.

The refractive index throughout a glass source has variation and the degree of this variation depends on the type of glass. For float glass, some studies have determined this variation to be 0.000033 [51]. Therefore when comparing a $\mathrm{K}$ and $\mathrm{Q}$, a minimum SD value of 0.000033 will be used. A Q glass chip will be considered indistinguishable in refractive index if it has a mean refractive index falling within $\pm 3 \mathrm{SD}$ of the mean of the refractive index of the $\mathrm{K}$ sample.

\subsection{Elemental Analysis}

As mentioned before, the main ingredient of glass is sand. Though there are many sand deposits around the world, only a small set is actually pure enough to be used in the formation of glass. Since the movement of sand from one area to another can be very costly, most glass manufacturers are located near a deposit of usable sand. This causes the composition of glass to vary by country/manufacturer. As shown in Table 12, the 
composition of the main components of float soda-lime glass varies by country especially in the concentrations of potassium, aluminum, iron, and barium.

Sensitive elemental techniques can be used to detect small variations in the chemical formulation between glasses that have been manufactured in different manufacturing plants or even at the same manufacturing plant at different time intervals. Elemental analysis of glass has been studied using SEM-EDS, uXRF, ICP-OES, ICP-MS, and LIBS. This research is focused on the analysis using LA-ICP-MS, so this technique will be described in detail in the following section.

Table 12 - Average values for the composition of float glass (\% element by weight)

\begin{tabular}{|lllllllll|}
\hline Country & Si & Na & Ca & Mg & K & Al & Fe & Ba \\
\hline UK & 34.08 & 9.50 & 5.86 & 2.29 & 0.66 & 0.74 & 0.07 & \\
US & 33.90 & 10.24 & 6.41 & 2.29 & 0.11 & 0.18 & 0.12 & 0.01 \\
Germany & 33.58 & 10.14 & 6.60 & 2.40 & 0.13 & 0.28 & 0.16 & 0.12 \\
Spain & 33.46 & 10.24 & 6.62 & 2.37 & 0.21 & 0.37 & 0.07 & \\
Japan & 33.54 & 9.66 & 5.80 & 2.36 & 0.83 & 0.90 & 0.14 & \\
\hline
\end{tabular}

LA-ICP-MS is a sensitive, micro-destructive elemental technique that requires

little to no sample preparation, thus making it a very powerful tool for forensic scientists. The basics of LA-ICP MS are the focusing of a laser on the surface of the sample, using a CCD camera. Under optimized parameters, the laser is fired at the sample located within an ablation cell. The laser ablates the sample producing small particles. A carrier gas, usually helium, transports the particles from the ablation cell to the ICP ionization source. In the following sections, the principles of lasers and ICP-MS instruments will be discussed as well as what is currently in use by forensic labs. 


\subsubsection{Principles of Lasers}

The term laser is actually an acronym that stands for light amplification by stimulated emission of radiation. Unlike most other light sources, laser beams are monochromatic (one wavelength) and coherent (waves are in phase with one another).

The typical components of a laser are an active medium, excitation mechanism, and optical resonator. The laser undergoes four processes: pumping, spontaneous emission, stimulated emission, and absorption. During the pumping process, the excitation source such as radiation from an external source, an electrical current, or discharge is used to excite the species in the active medium. The excited species may undergo spontaneous emission (fluorescence), which produces incoherent photons, which travel out of the material. The excited species will also undergo stimulated emission, which is caused when a photon interacts with an excited particle and causes the relaxation of the species through the emission of a photon that is the same energy and in phase with the photon that caused it to be emitted, coherent. As these photons pass through the active medium, which is encased in the optical resonator that causes the photons to move back and forth, they continue to interact with particles and can cause stimulated emission if the particle was in an excited state or are absorbed by a ground state particle. In order to have an amplification of light, the number of photons emitted must be greater than the number absorbed. This can only occur if more particles are in the excited states than in the ground state, population inversion. This can be achieved through pumping [37]. 


\subsubsection{Principles of ICP-MS}

Though a variety of ICP-MS manufacturers exist, they all generally operate the same way. A plasma is created when argon flowing through a quartz tube is ionized by colliding with highly energetic free electrons, which are initially generated by a spark from a Tesla coil. Once initiated, the plasma is stabilized by a magnetic field that is generated by applying an RF current to a copper coil that is located below the mouth of the quartz tube. The particles are carried through the center of the plasma where they are vaporized, atomized, excited, and ionized by a variety of mechanisms, usually by colliding with ionized and excited argon species [53]. Only the ionic species are used for analysis. These are extracted into the vacuum-regulated mass spectrometer by passing through sampling and skimmer cones. The ions then travel through a series of lens, which help to separate out neutral atoms and photons as well as focus the ions into a beam. Next, the ions are separated by their mass to charge ratio by traveling through a mass analyzer. There are a variety of mass analyzers available that differ by resolution, the ability to separate different ions with similar mass to charge ratios, and the principles used to separate ions. The quadrupole is one of the simplest and cheapest mass analyzers and is the main mass analyzer used in this research. The quadrupole is made up of four parallel rods with oscillating electric fields. The quadrupole, however, is only able to separate ions one mass unit apart. This causes the element menu to be further restricted to only the isotopes that are free from isobaric and polyatomic interferences. For example: the ${ }^{40} \mathrm{Ca}$ isotope should not be used because of large ${ }^{40} \mathrm{Ar}$ interferences. ${ }^{56} \mathrm{Fe}$ should not be used because of polyatomic interferences caused by ${ }^{40} \mathrm{Ar}^{16} \mathrm{O}$. The sorted ions then travel to an electron multiplier detector where an electrical current is generated and 
amplified. Because ICP-MS only detects ions, the possible elemental menu is limited to only those elements that have a first ionization potential less than that of argon [54].

There are a variety of lasers and ICP-MS instruments available on the market. One major distinction between different types of lasers is the active medium, the material used to create the monochromatic radiation. The active medium can be a crystal, semiconductor, organic dye, or a gas. A brief survey sent to some forensic LA-ICP-MS laboratories revealed that these labs use either a crystal or gas as an active medium, Table 13. When it comes to crystals, all forensic labs surveyed use a Neodymium Yttrium Aluminum Garnet (Nd:YAG) laser that can be altered to create a variety of wavelengths $(1064 \mathrm{~nm}, 532 \mathrm{~nm}, 355 \mathrm{~nm}, 266 \mathrm{~nm}, 213 \mathrm{~nm})$. The wavelengths in the UV range are used for glass analysis because these have been shown to couple nicely to the glass matrix. The other forensic labs surveyed used lasers with gas active mediums, specifically excimer lasers that used a mixture of argon and fluorine gas. These lasers operate at a wavelength of $193 \mathrm{~nm}$.

Other properties of lasers that are optimized for the forensic analysis of glass are the laser energy, frequency, and spot size. The frequency is defined as the number of shots per second. The maximum frequency differs by laser manufactures, but most forensic labs use a frequency of $10 \mathrm{~Hz}$. All labs surveyed used a spot size less than 100um. This is because glass is very homogenous so a large spot size is not needed and because Q glass fragments are very small in size. A smaller spot size allows for more replicate measurements to be performed.

The evaluation of the analytical performance of different combinations of laser ablation systems with different ICP-MS instrument was one of the Natural Isotopes and 
Trace Elements in Criminalistics and Environmental Forensics (NITECRIME) European Network main objectives. This group identified optimal laser ablation and ICP-MS acquisition parameters for glass analysis. Even though the instrumentation differed, they determined that when using matrix matched glass standards LA-ICP-MS can deliver quantitative measurements on major, minor and trace elements in float glass samples for forensic and other purposes [42]. 
Table 13 - LA-ICP-MS instrumentation used by laboratories located around the world

\begin{tabular}{|c|c|c|c|c|c|c|c|}
\hline Laser & & & & & & & \\
\hline Make & New Wave & New Wave & $\begin{array}{l}\text { Applied Spectra } \\
\text { J200 Tandem }\end{array}$ & New Wave & New Wave & New Wave & $\begin{array}{l}\text { Applied Spectra } \\
\text { J200 Tandem }\end{array}$ \\
\hline Wavelength (nm) & 213 & 213 & 266 & 193 & 193 & 213 & 226 \\
\hline Frequency (Hz) & 10 & 10 & 10 & 10 & & 10 & 10 \\
\hline Spot Size $(\mu \mathrm{m})$ & 55 & 90 & 50 & 80 & 40 & 65 & 30 \\
\hline \multicolumn{8}{|l|}{ ICP-MS } \\
\hline \multirow{2}{*}{ Make } & Thermo & Perkin Elmer & Perkin Elmer & Agilient & Thermo & Agilent & Analytik Jena \\
\hline & X Series II & ELAN DRC & Nexion & $7700 x$ & IcapQ & $7500 \mathrm{cx}$ & PlasmaQuant MS Elite \\
\hline
\end{tabular}




\subsection{Comparison Criteria for LA-ICP-MS measurements}

There have been many studies focused on testing different match criteria to see which one is most appropriate for forensic glass comparisons using (LA) ICP-MS [43-46, 55-59]. The match criteria that have been heavily explored include the T-test with an $\alpha$ of 0.05, 0.01, and Bonferroni correction, match intervals created with $\pm 2,3,4,5$, and 6 standard deviations of the known sample, match intervals created with $\pm 2,3,4,5$, and 6 standard deviations but setting a minimum standard deviation equal to $3 \%$ of the mean, Hotelling's $\mathrm{T}^{2}$, and range overlaps. When evaluating the performance of a statistical test for forensic comparisons, there are two factors that are used, false exclusion and false inclusion error rates. A false exclusion usually refers to failing to associate samples with common origin; whereas a false inclusion is failing to discriminate samples that originated from different sources.

The Elemental Analysis Working Group (EAWG) made up of forensic glass examiners and research scientists was formed to develop robust analytical protocols and to assess the accuracy of various statistical tests used for source comparison. The group went through four inter-laboratory tests that measured the error rates of selected match criteria on glasses having very similar elemental compositions. For each match criteria, the false exclusion and false inclusion error rates were calculated and used to evaluate the performance of the statistical test. The EAWG's inter-laboratory tests are explained in more detail in 7.8

For LA-ICP-MS and ICP-MS, the EAWG suggested using a modified \pm 4 standard deviations (SD) for the comparison between a K and Q sample[60]. Using the K, 
the mean and SD for each element are calculated. A minimum SD equal to at least 3\% of the mean for each element is also calculated. This is sometimes referred to as the fixed relative standard deviation (FRSD). If the SD is less than the FRSD, the FRSD is used for creating a comparison interval. The $\mathrm{K}$ comparison interval is calculated for each element as the mean $\pm 4 \times \mathrm{SD}$ (or $\pm 4 \times \mathrm{FRSD}$, whichever is greatest). The average concentration for each element of the Q sample is then calculated and compared to the $\mathrm{K}$ comparison interval. If the $\mathrm{Q}$ average lies outside the comparison interval for any element, then the $\mathrm{K}$ and Q are distinguishable. This match criterion has been published in ASTM E2927 [60].

\subsection{Interpretation of Glass Evidence}

\subsubsection{Verbal Scale}

Some forensic laboratories are currently using a verbal scale to help report their findings to a jury. These verbal scales are divided by the presence or absence of individual or class characteristics as well as the discrimination potential of the techniques used [48]. One such verbal scale was presented by Chris Bommarito at the 2009 Trace Evidence Symposium. This scale is listed below and taken from Bommarito's presentation.

- “Type I Association: A positive identification; an association in which items share individual characteristics that show that the items were once from the same source.

- Type II Association: An association in which items are consistent in all measured physical properties and/or chemical composition and share unusual 
characteristic(s) that would not be expected to be found in the population of this evidence type.

- Type III Association: An association in which items are consistent in all measured physical properties and/or chemical composition and could have originated from the same source. Because similar items have been manufactured and would be indistinguishable from the submitted evidence, an individual source cannot be determined.

- Type IV Association: An association in which items are consistent in measured physical properties and/or chemical composition. This sample type is commonly encountered in our environment and may have limited associative value.

- Type V Association: An association in which items are consistent in some, but not all, physical properties and/or chemical composition. Some minor variation exists between the known and questioned items and could be due to factors such as sample heterogeneity or contamination of the sample(s).

- Inconclusive: No conclusion could be reached regarding an association between the items.

- Elimination: The items were dissimilar in physical properties and/or chemical composition and did not originate from the same source. This approach may be considered as subjective since it relies on the analyst's personal experience and assessment of the glass evidence [48]."

Scientists, however, do not always agree on what category evidence falls in. There is also no standard verbal scale and usually each forensic lab uses their own. Some of these scales take into account the activity level of the suspect, such as the number of 
glass fragments found. Others like the Bommarito scale presented here only look at the source level of the glass. Therefore, this approach may be considered as subjective since it relies on the analyst's personal experience and assessment of the glass evidence.

\subsubsection{Random Match Probability}

A random match probability is defined here as the probability of two glass samples from different sources being indistinguishable in elemental composition. Some laboratories estimate the random match probability as the false inclusion rate of a comparison criterion. Today, the reported error rate for most LA-ICP-MS generated glass databases is approximately $0.1 \%[45,46]$.

In this study, different comparison criteria were tested to determine which one correctly associates glass duplicate samples ran months apart while still providing the ability to discriminate samples from different vehicles.

\subsubsection{Frequency}

Another statistic calculated from databases is the frequency of an elemental composition. Unlike the random match probability, the frequency is defined here as how many times a specific elemental composition is encountered in a database i.e. how many samples in the database are indistinguishable with the $\mathrm{K}$ from a case. The match criteria used to calculate the random match frequency was also used here to compare a specific sample to a database. 


\section{CHAPTER 7. Creating the M\&M Salvage Yard Glass Database}

\subsection{Glass Sample Set}

\subsubsection{Reference Standards}

The standard reference materials SRM NIST612 and SRM NIST1831 (National

Institute of Standards and Technology, Gaithersburg, MD, USA) were used as well as matrix-matched float glass standards FGS1 and FGS2 (Bundeskriminalamt, Wiesbaden, Germany).

Table 14 shows the elemental values for the references glasses used in this study. For the NIST glasses, the reported values used were from ASTM E2927 [60]. For the FGS glasses, the reported values used were from Latkoczy et al [42]. 
Table 14 - Concentrations (ppm) of elements in reference glasses

\begin{tabular}{|llllllllll|}
\hline & Li7 & Mg25 & Al27 & K39 & Ca42 & Ti49 & Mn55 & Fe57 \\
\hline NIST612 & 40.0 & 68.0 & 10743.8 & 62.3 & 85048.2 & 44.0 & 37.7 & 51.0 \\
FGS2 & 29.0 & 23400.0 & 7400.0 & 4600.0 & 59300.0 & 326.0 & 221.0 & 2600.0 \\
NIST1831 & 5.0 & 21200.0 & 6380.0 & 2740.0 & 58600.0 & 114.0 & 13.1 & 608.0 \\
FGS1 & 6.0 & 23900.0 & 1500.0 & 920.0 & 60600.0 & 69.0 & 43.0 & 580.0 \\
\hline
\end{tabular}

\begin{tabular}{|lccccccccc|}
\hline & Rb85 & Sr88 & Zr90 & Ba137 & La139 & Ce140 & Nd146 & Hf178 & Pb208 \\
\hline NIST612 & 31.4 & 78.4 & 37.9 & 38.6 & 36.0 & 38.4 & 35.5 & 36.7 & 38.6 \\
FGS2 & 35.0 & 253.0 & 223.0 & 199.0 & 18.0 & 23.0 & 25.0 & 15.0 & 24.0 \\
NIST1831 & 6.1 & 85.0 & 36.0 & 31.5 & 2.1 & 4.5 & 1.8 & 1.0 & 1.8 \\
FGS1 & 8.6 & 57.0 & 49.0 & 40.0 & 4.3 & 5.2 & 5.1 & 3.2 & 5.8 \\
\hline
\end{tabular}




\subsubsection{M\&M Salvage Yard}

The M\&M Salvage Yard (Ruckersville, VA) was selected for sample collection because this is the salvage yard that the Insurance Institute of Highway Safety uses to dispose of their vehicles after their safety rating tests. This means that this salvage yard contains a wide variety of relatively new vehicles with OEM windshields as well as older vehicles.

Cars with a broken windshield were sampled. A windshield cutter (RHYNO, Pittsburgh, PA) was used to cut a 5-8 cm square out of each windshield. Where possible, three squares were taken. A numbered sticker, 1-210, was attached to the pane of glass that was exposed to the outside. If the window marking was legible, a photo was taken, Figure 31.

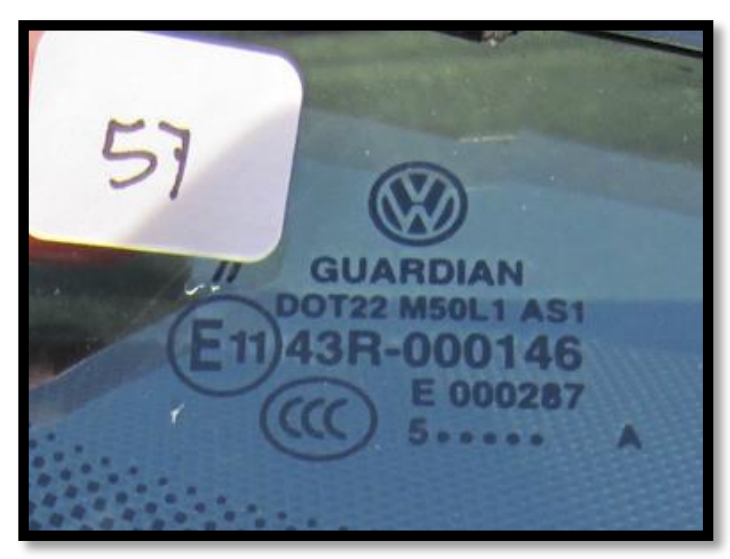

Figure 31 - An example of a window marking found on the windshield of a vehicle

A total of 210 cars were sampled. The years of the vehicles ranged from 20042017 and 26 car makes were represented. The distribution of years and makes can be seen in Figure 32 and Figure 33. 


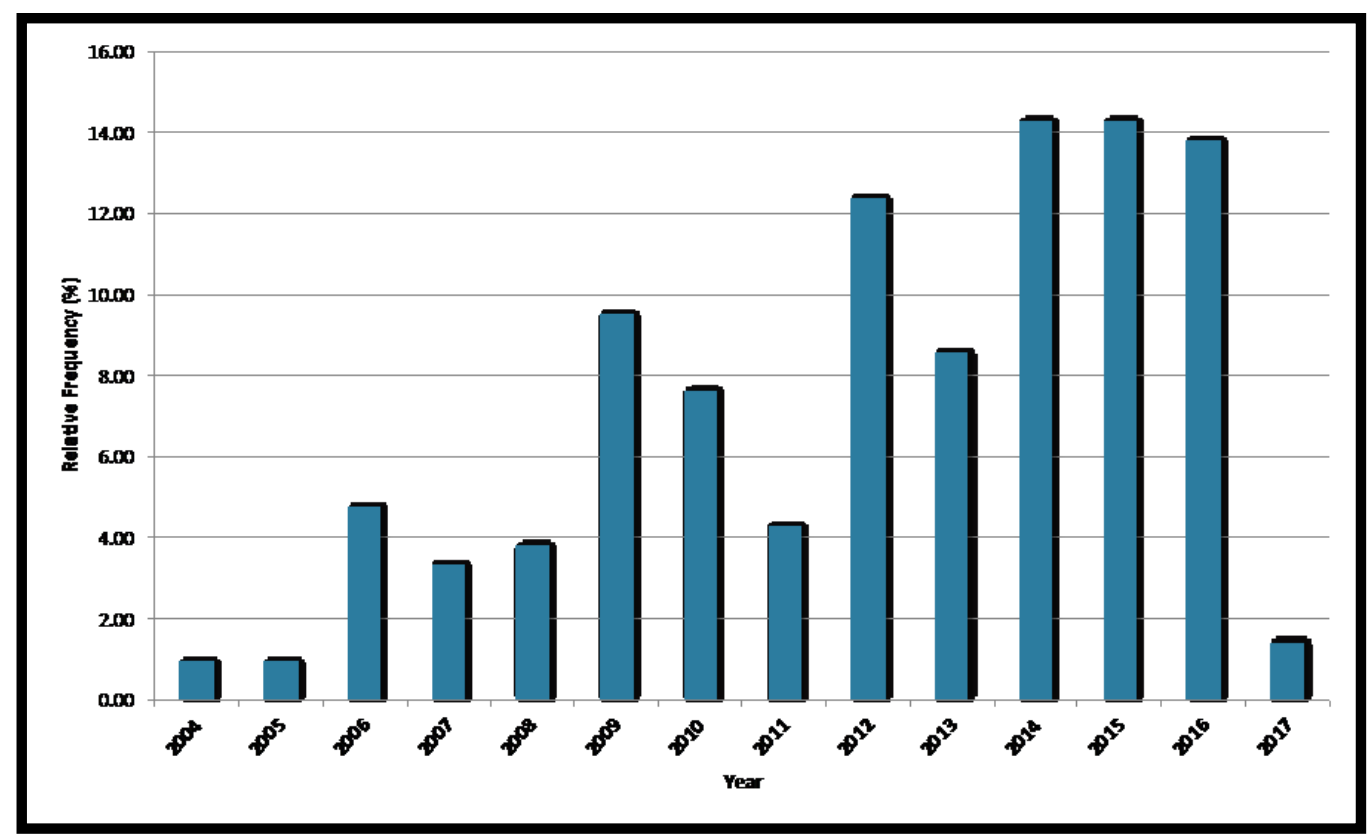

Figure 32 - Distribution of the years of vehicles sampled

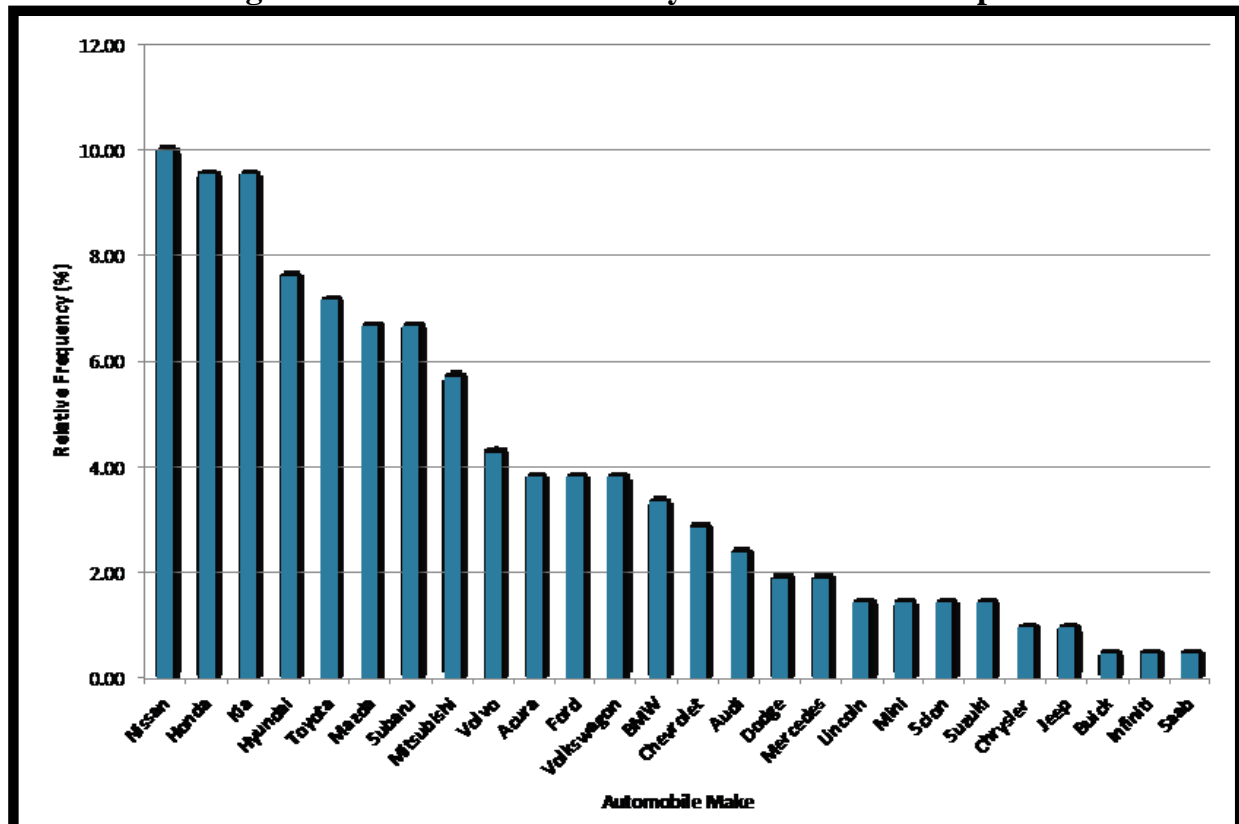

Figure 33 - Distribution of the makes of vehicles sampled

7.2 Preparation of Glass Samples for LA-ICP-MS

Each 5-8 cm square of laminated glass from M\&M Salvage Yard was wrapped in

3 sheets of copy paper. While completely wrapped, a large pestle was used to fragment 
the glass panes. A black marker was used to draw a $\bullet$ on multiple fragments on the outer pane. Three fragments were selected and carefully removed from the polymer film, wrapped in weighing paper, and placed in a pill box labeled with the sample number and "Outer". A purple marker was used to draw an $\times$ on multiple fragments on the inner pane. Three full thickness fragments were selected and carefully removed from the polymer film, wrapped in weighing paper, and placed in pill box labeled with the sample number and "Inner".
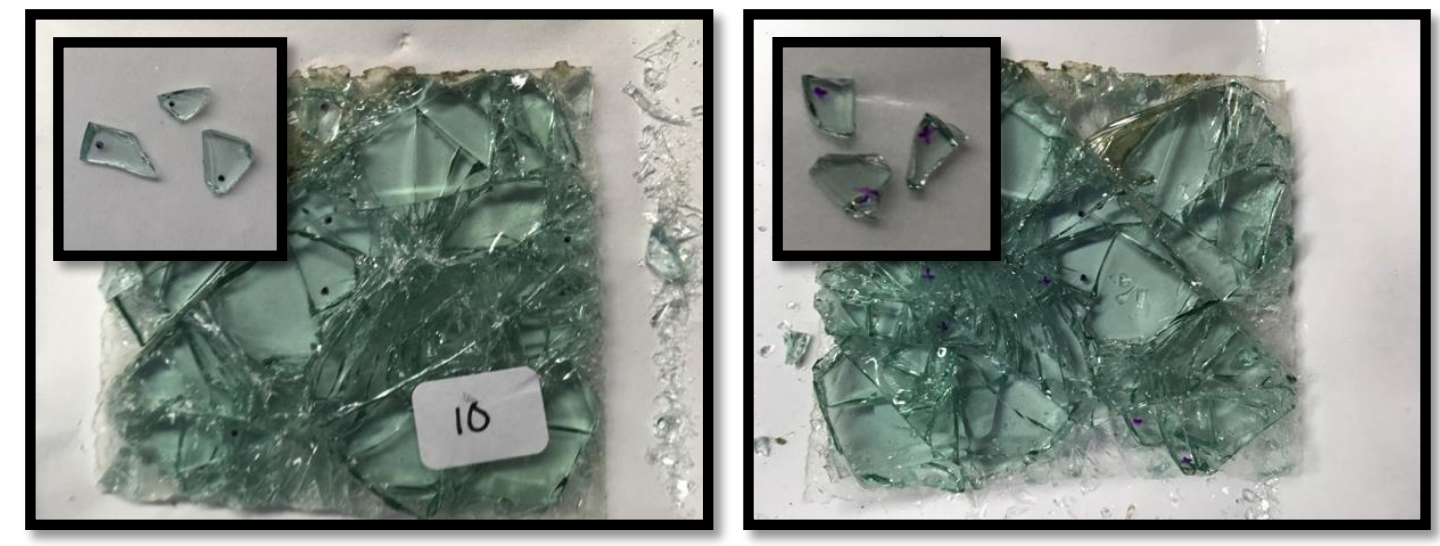

Figure 34 - Sample preparation for LA-ICP-MS

\subsection{Analysis with LA-ICP-MS}

Laser ablation ICP-MS was performed using a quadrupole ELAN DRC II 6100 (Perkin Elmer LAS, Shelton, CT) connected to a $213 \mathrm{~nm}$ laser ablation system (New Wave Research Inc., Fremont, CA). The laser parameters were optimized and validated for casework by a previous student. The parameters were as follows: $100 \%$ energy $(0.65$ $\mathrm{mJ}), 10 \mathrm{~Hz}, 90 \mu \mathrm{m}$ spot, $60 \mathrm{~s}$ dwell. Helium at $0.9 \mathrm{~L} /$ minutes was used as the ablation and carrier gas. The ICP-MS parameters were RF power of $1500 \mathrm{~W}, 8.3 \mu$ s dwell time, peak hopping mode, and 2 sweeps per reading. The number of readings was set at 220 . This 
allowed for the collection of a $20 \mathrm{~s}$ gas blank, a $60 \mathrm{~s}$ ablation signal, and $20 \mathrm{~s}$ cleanup. The following isotopes were monitored in time resolved analysis mode: ${ }^{7} \mathrm{Li},{ }^{25} \mathrm{Mg},{ }^{27} \mathrm{Al}$, ${ }^{29} \mathrm{Si},{ }^{39} \mathrm{~K},{ }^{42} \mathrm{Ca},{ }^{57} \mathrm{Fe},{ }^{49} \mathrm{Ti},{ }^{55} \mathrm{Mn},{ }^{85} \mathrm{Rb},{ }^{88} \mathrm{Sr},{ }^{90} \mathrm{Zr},{ }^{118} \mathrm{Sn},{ }^{137} \mathrm{Ba},{ }^{139} \mathrm{La},{ }^{140} \mathrm{Ce},{ }^{146} \mathrm{Nd},{ }^{180} \mathrm{Hf}$, ${ }^{208} \mathrm{~Pb}$.

Both the laser and ICP-MS were warmed up for at least an hour prior to analysis. After the warm-up, daily performance was performed each day using NIST SRM 612 (Trace Elements in Glass Matrix) (NIST, Gaithersburg, MD). The counts for ${ }^{7} \mathrm{Li},{ }^{139} \mathrm{La}$, ${ }^{140} \mathrm{Ce},{ }^{21} \mathrm{Ca}^{++} / 42 \mathrm{Ca},{ }^{248} \mathrm{ThO} /{ }^{232} \mathrm{Th}$, were monitored and compared to previous days to make sure the instrument did not need maintenance.

The sample analysis for each day was: FGS2, Sample 1 inner pane, Sample 1 outer pane, Sample 2 inner pane, Sample 2 outer pane, NIST1831, Sample 3 inner pane, Sample 3 outer pane, Sample 4 inner pane, Sample 4 outer pane, FGS2, Sample 5 inner pane, Sample 5 outer pane, Sample 6 inner pane, Sample 6 outer pane, FGS1, Sample 7 inner pane, Sample 7 outer pane, Sample 8 inner pane, Sample 8 outer pane, FGS2.

Five replicate measurements were taken on each sample glass fragment (15 total measurements for each sample pane). Five replicates were taken on each reference glass. FGS2 was analyzed during the beginning, middle, and end of each day to better account for instrumental drift.

\subsection{Data Analysis}

After LA-ICP-MS analysis, signal processing was accomplished using GLITTER (MacQuarie University, Australia). An example of the integration strategy is shown in, Figure 35. The first few seconds of laser-sample interaction were ignored. The software 
integrated the signal, subtracted the gas blank signal, and normalized the data ${ }^{29} \mathrm{Si}$. The GLITTER software also corrected for instrumental drift using linear yield to ratio and calculated the concentration of each isotope in each of the replicate measurements using concentration values for FGS2. Sometimes, a spike in the signal will occur. These spikes could be caused by difference in the particle size causing only partial vaporization of the sample. During integration of the signal, spikes that were only one reading long were removed by using the filtering button. This button averaged the reading before and after the spike to reduce the spike to a more plausible value. 


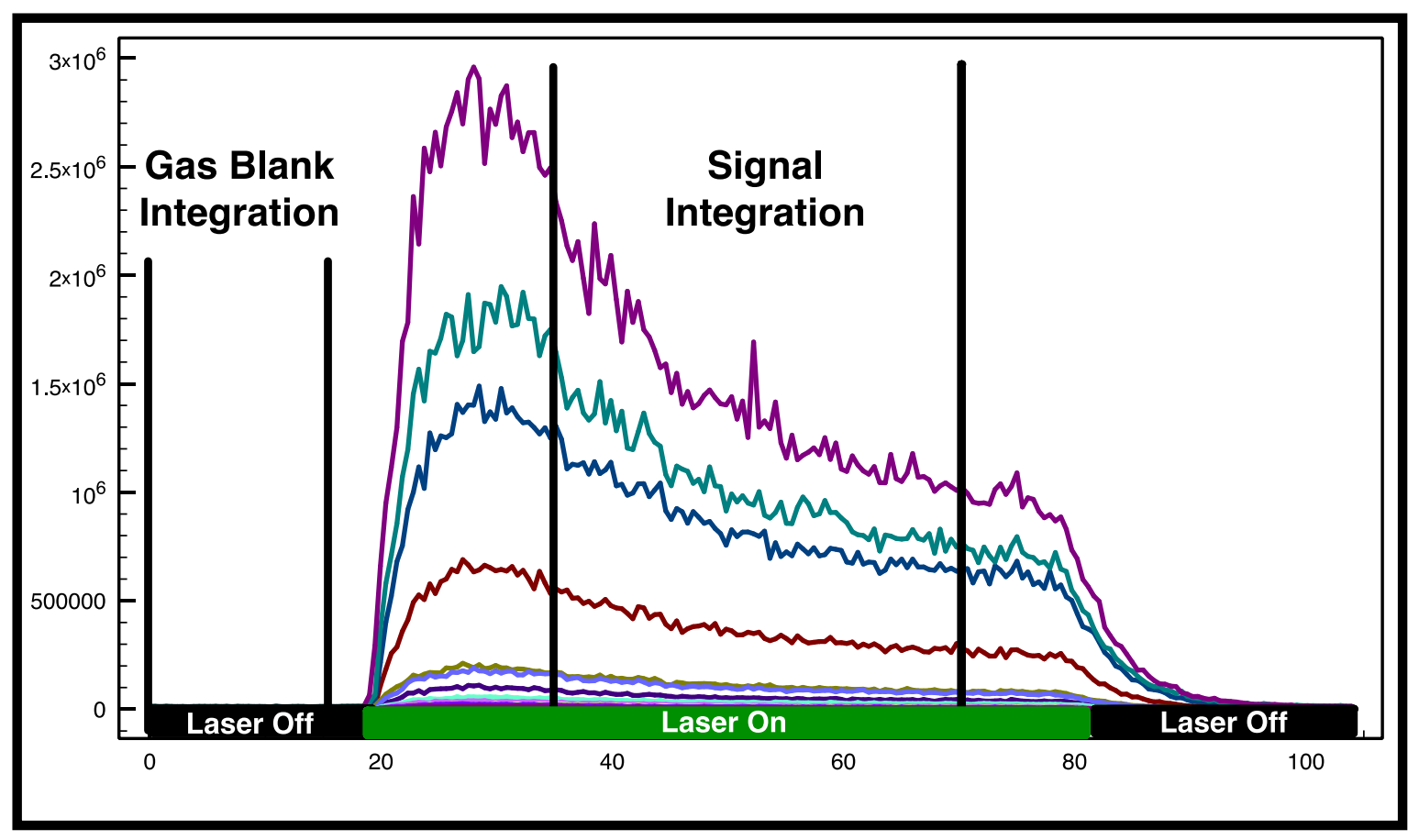

Figure 35 - Integration strategy used for glass

7.5 Statistical Analysis

\subsubsection{FGS1 and NIST1831 Control Charts}

FGS1 and NIST1831 were analyzed on every day of analysis. NIST1831 was analyzed as a quality control for the morning while FGS1 was analyzed during the afternoon, see section 2.3. Present in this section are the quality control charts for the NIST1831 control glass. The FGS1 control charts can be found in the Appendix.

The control charts were created using the Levey Jennings style. The average for each measurement is plotted as a point along with the standard deviation. The green line is the overall average. The dotted yellow lines are equal to the overall average \pm the overall standard deviation $\times 2$ and represent warning lines. The red dotted lines are equal to the overall average \pm the overall standard deviation $\times 3$ and represent the acceptable 
upper and lower limit. If a point falls outside the red lines, further investigation was done. The solid blue line is the reported concentration in NIST1831 according to ASTM 2927 [60]. For $\mathrm{Li}, \mathrm{Mg}, \mathrm{K}, \mathrm{Fe}, \mathrm{Ti}$ and $\mathrm{Rb}$ the reported value is different than the overall average and falls either near the yellow warning line or outside the upper or lower limits. This might is most likely caused by inaccuracies in the reported concentration for the calibrator, FGS2. According to a scientist at the BKA, the values for FGS2 and FGS1 are going to be updated. The updated values will be based on new inter-laboratory tests and/or additional elemental analyses of the glass using neutron activation analysis. 

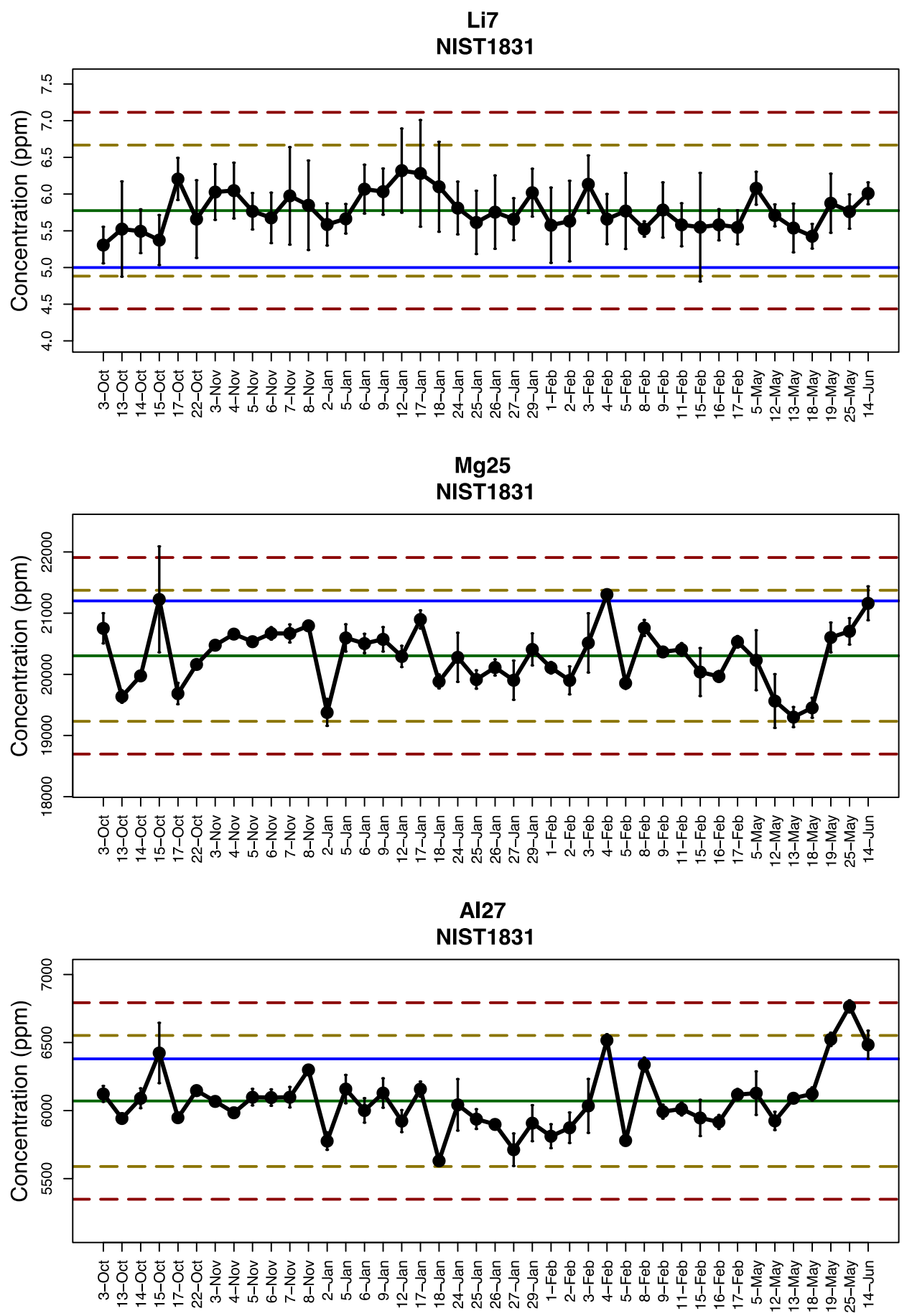

Figure 36 - Levey Jennings control charts for NIST1831 

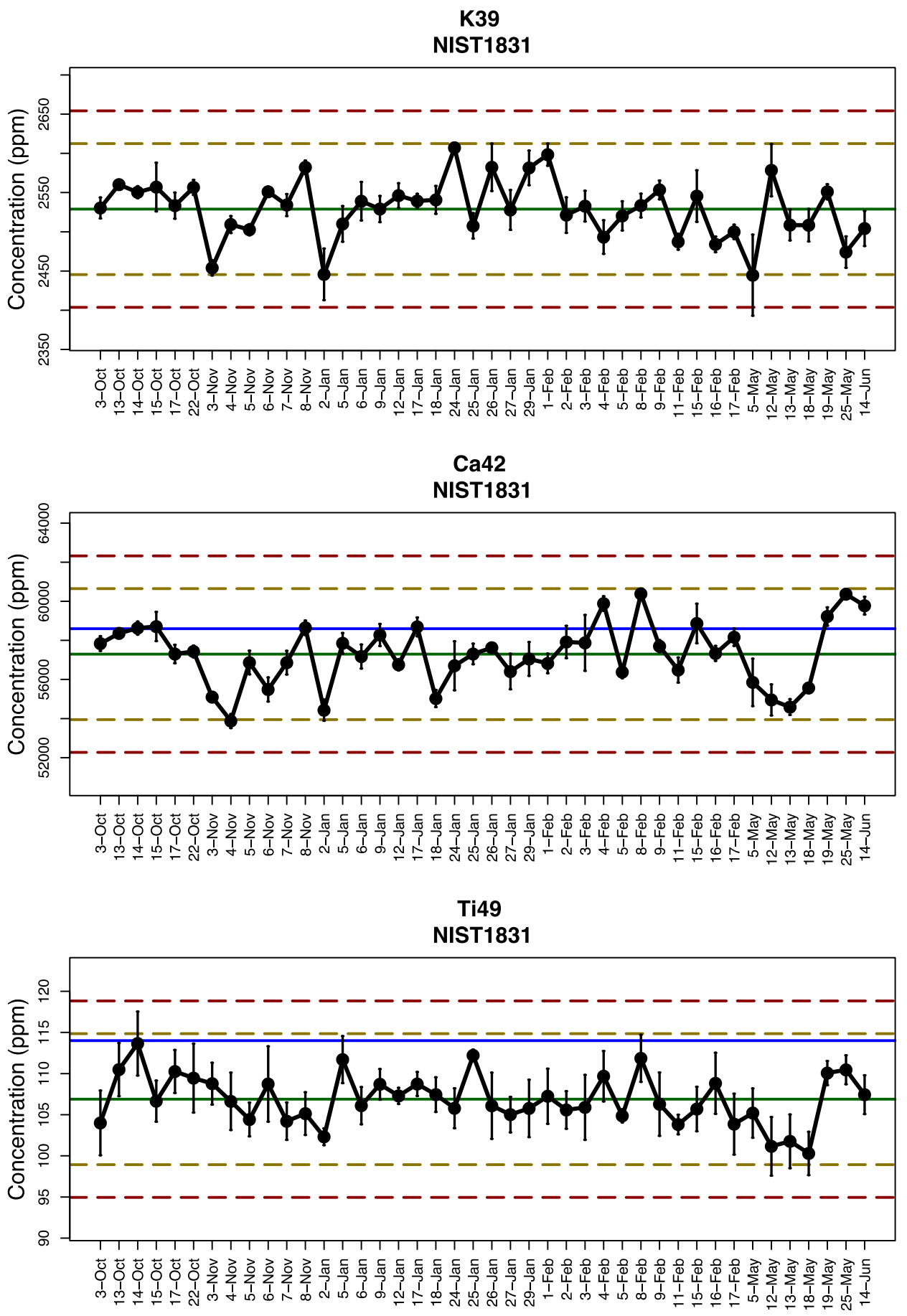

Levey Jennings control charts for NIST1831 (continued) 

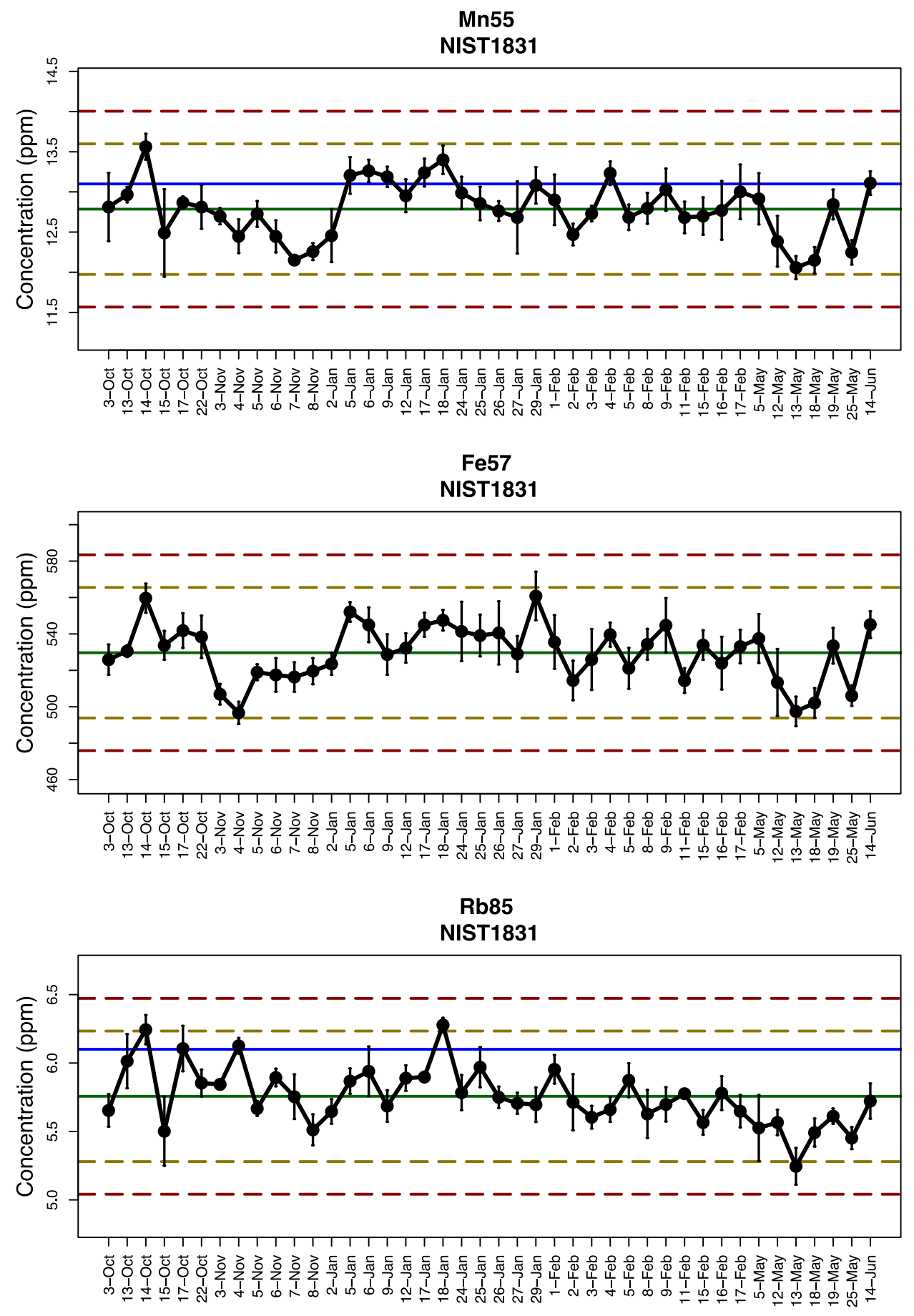

Levey Jennings control charts for NIST1831 (continued) 

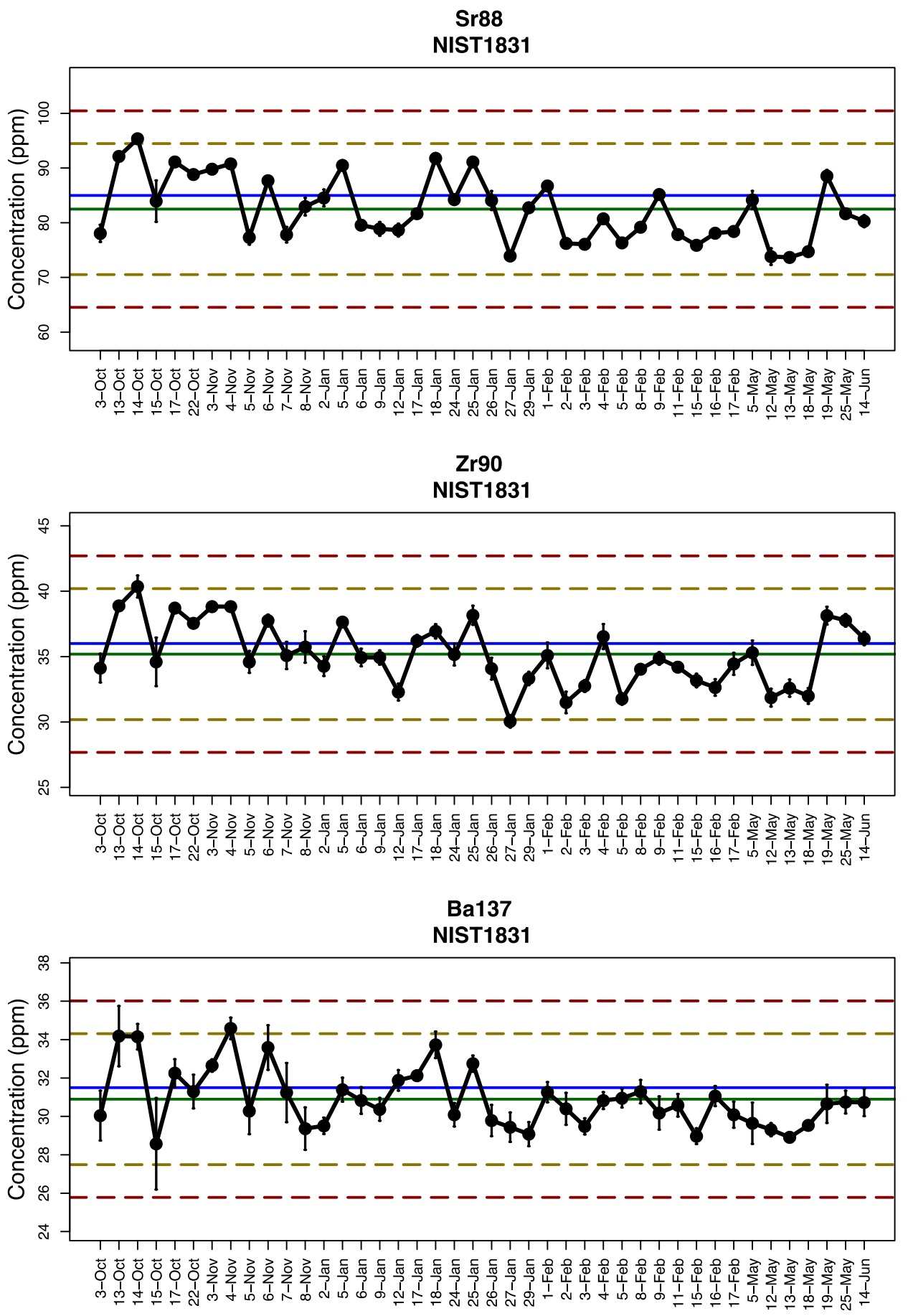

Levey Jennings control charts for NIST1831 (continued) 

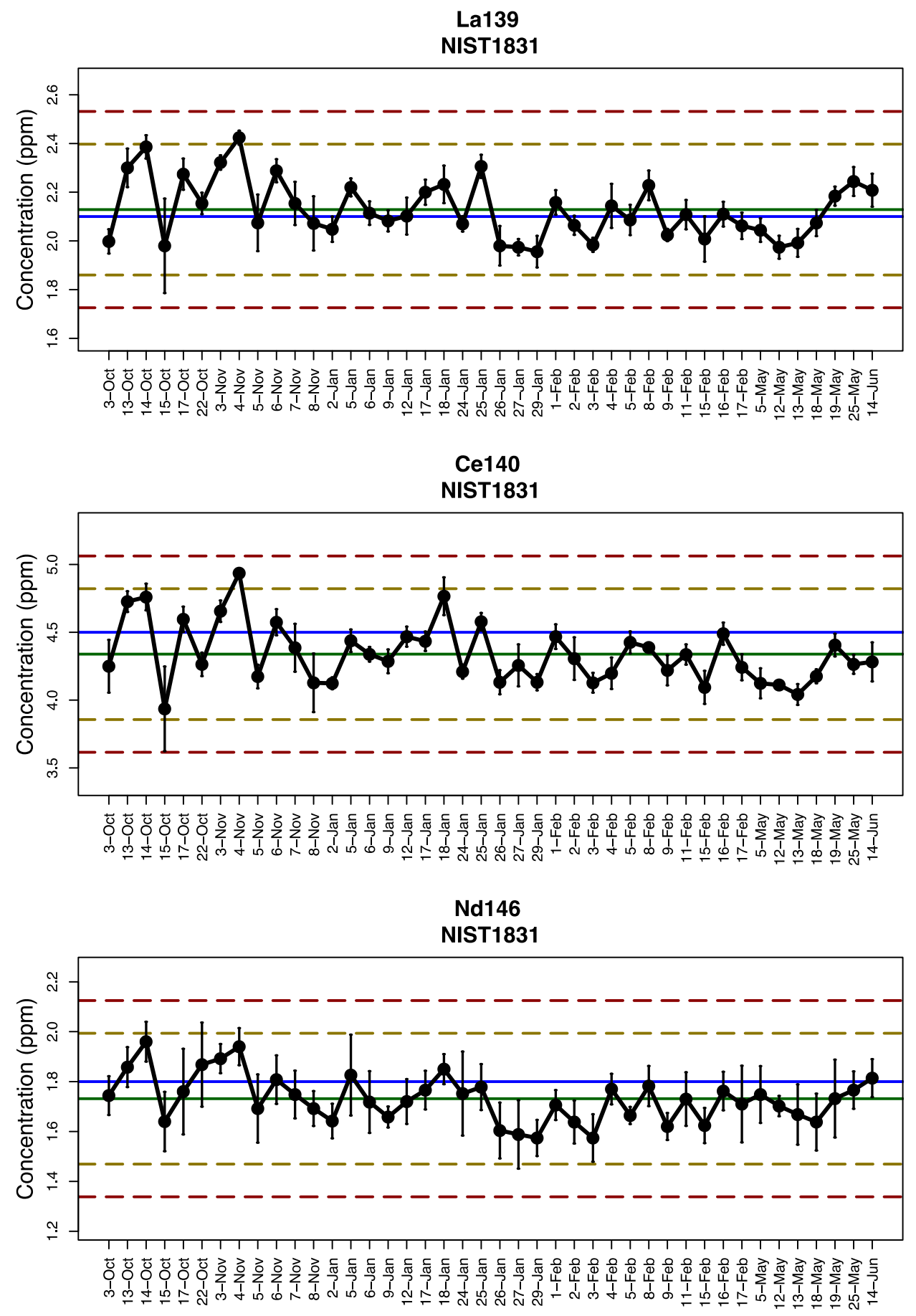

Levey Jennings control charts for NIST1831 (continued) 

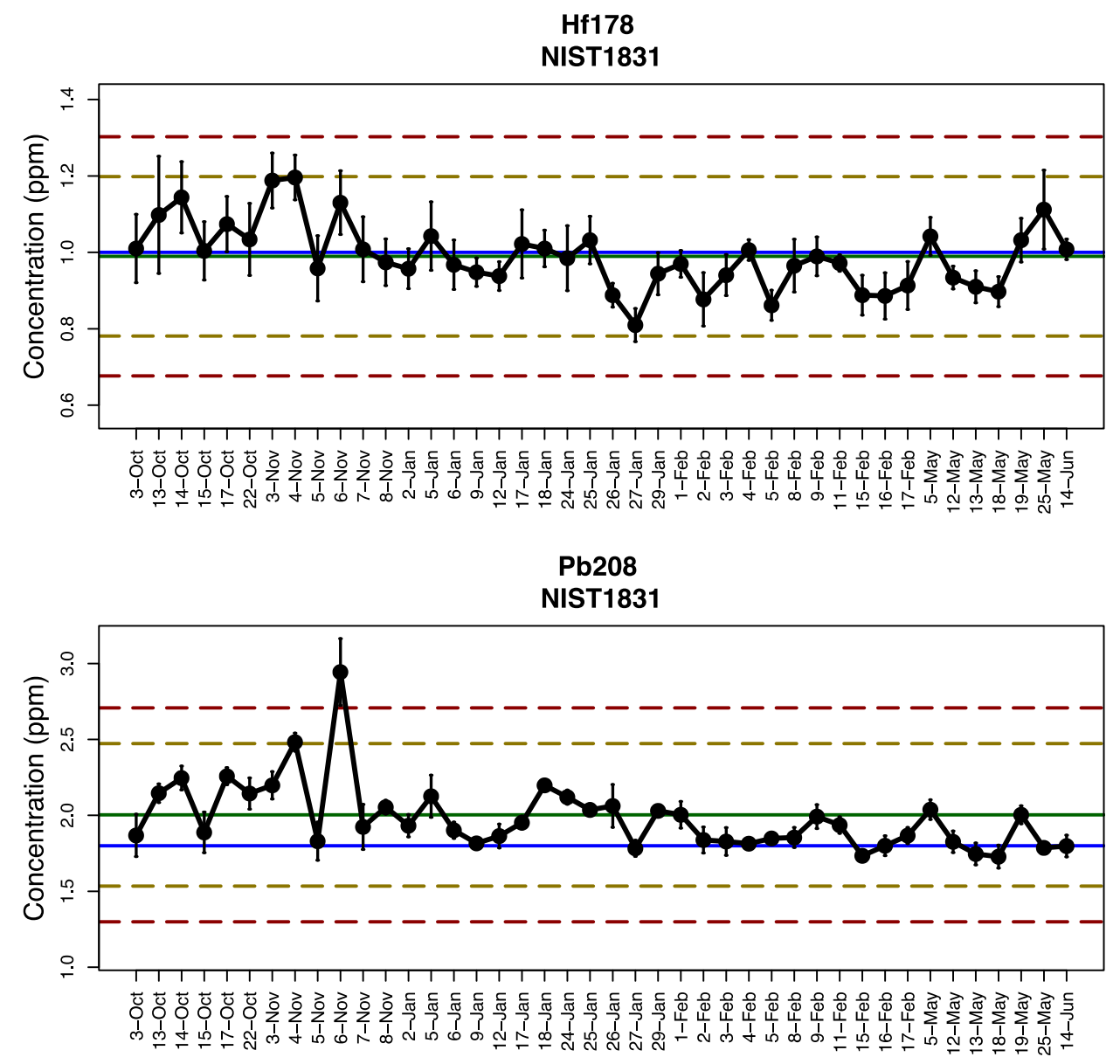

Levey Jennings control charts for NIST1831 (continued)

\subsubsection{Thickness Measurements}

Using a micrometer, the thickness of each glass fragment was measured. The average was recorded. The average thickness can be found in the Appendix. Each sample was handled as a $\mathrm{K}$ and used to create a comparison interval equal to the $\mathrm{K} \pm 0.15 \mathrm{~mm}$. The other samples would then be treated as Qs and if the thickness of these samples fell within the comparison interval, the samples were indistinguishable. Of the possible 175980 comparisons, 74182 (42\%) were distinguished by thickness. 


\subsection{Simplifying the Database}

\subsubsection{Removing Similar Panes}

One thing that was noticed about the M\&M vehicle database is that some samples $(\sim 20 \%)$ had different colored inner and outer panes, which resulted in dissimilar elemental profiles. While others had inner and outer panes that were the same color and had very similar elemental compositions. Having panes with similar elemental profiles makes determining error rates difficult. For example, if one pane is found to be indistinguishable with another source, the other pane is most likely going to be indistinguishable. This causes an increase in the false inclusion error rate. Also from a forensic standpoint, if a Q sample was found to be indistinguishable with both the $\mathrm{K}$ inner and $\mathrm{K}$ outer taken from the windshield of a suspect vehicle, the scientist usually reports that no differences were found therefore the $\mathrm{Q}$ could have originated from the same source as the $\mathrm{K}$ i.e. the windshield of the suspect car. When forensic glass examiners were asked how they treat vehicles with similar inner and outer panes, in their databases, they stated that only one pane is added to the database.

Since the inner and outer pane were analyzed on the same day, the ASTM E2927 comparison criteria with a FRSD of $3 \%$ for all elements was used to compare inner panes to outer. If the pairs were found to be indistinguishable, the outer pane was removed. The reduced the M\&M database from 420 samples to 333. This reduced database was used for the remainder of this study. 


\subsubsection{Limiting the Element Menu}

Forensic scientists participating in the GIWG have said that ${ }^{90} \mathrm{Zr}$ and ${ }^{178} \mathrm{Hf}$ are very correlated. Some of these scientists removed ${ }^{178} \mathrm{Hf}$ from their database and others talked about no longer analyzing ${ }^{178} \mathrm{Hf}$. The correlation of elements within the FIU M\&M samples is shown in Figure 37. The darker the blue color, the more positively correlated two elements are. The darker the red color, the more negatively correlated two elements are. As can be seen, ${ }^{90} \mathrm{Zr}$ and ${ }^{178} \mathrm{Hf}$ are indeed correlated with a coefficient 0.997 . Therefore ${ }^{178} \mathrm{Hf}$ was removed from the database. There are indications of other elements that may be correlated such as: ${ }^{27} \mathrm{Al}$ with ${ }^{39} \mathrm{~K}$ and ${ }^{85} \mathrm{Rb},{ }^{130} \mathrm{La}$ with ${ }^{140} \mathrm{Ce}$ and ${ }^{148} \mathrm{Nd}$, and ${ }^{197} \mathrm{Ba}$ with ${ }^{208} \mathrm{~Pb}$, etc. These were not discussed with the other participants of the GIWG, and thus remained in the database. 


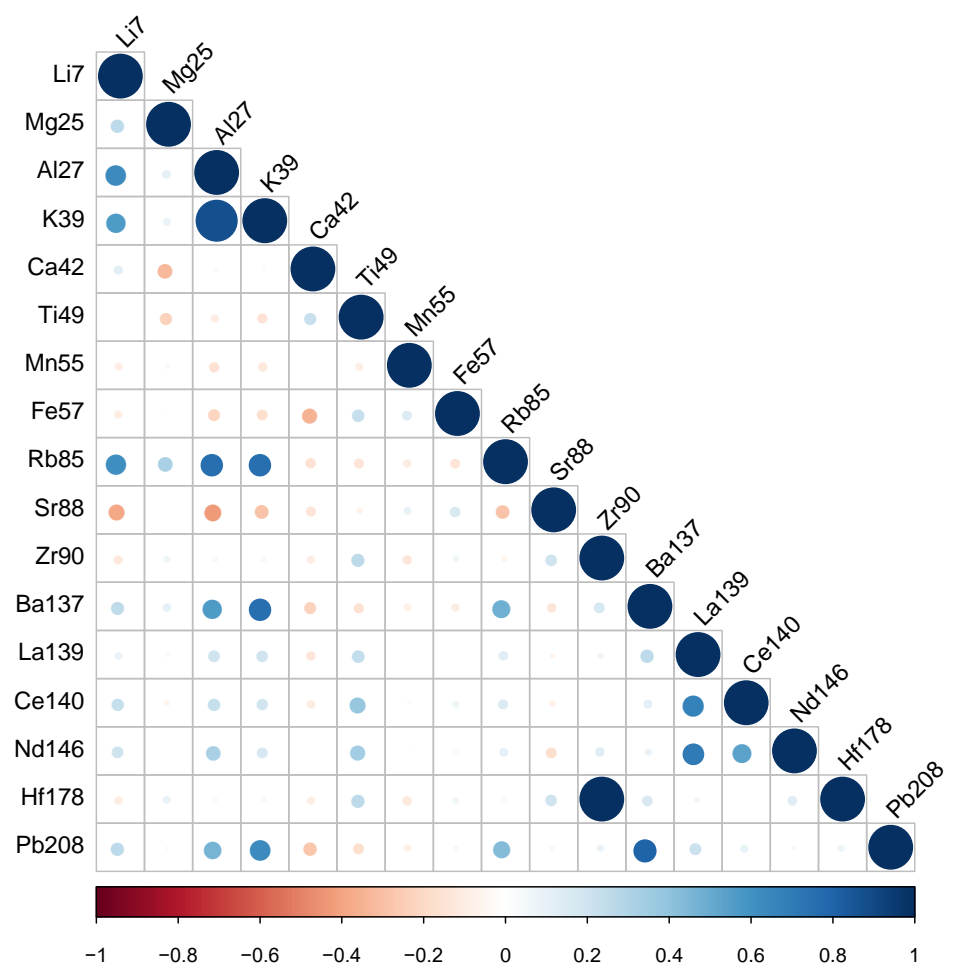

Figure 37 - Correlation of elements in the M\&M database

\subsection{Determining Random Match Probability and Frequency Comparison Criteria}

As the LA-ICP-MS system performs analyses day after day, the sampling and skimmer cones become dirty, the calibration of the pulse/analog mode of detector starts to shift, the laser energy begins to decrease, and maintenance needs to be done. All of these can cause slight variations in the concentration of samples run on different days. When comparing samples to a database, it is important that the inter-day variation is accounted for. However, ASTM E2927 states that the K and Q should be analyzed on the same day and does not mention how to incorporate inter-day variation into the comparison criteria. Fortunately, there are two laboratories have tested and evaluated different approaches to incorporate inter-day variation. 
One approach was to use the ASTM E2927 comparison criteria but assign a minimum FRSD that was based on the element's variation within the database. This group first calculated the elemental variation within two data sets: a single float glass pane and between different float glass sources. For each data set, the relative standard deviation (RSD) was calculated for each sample. The average RSD was used to help determine a minimum FRSD for each element. The lowest FRSD used was 4\%.

The other approach was to calculate a FRSD based on the inter-day variability of a control glass that was analyzed over the course of 90 days. For each day the average concentration of the control was calculated (mean of 3 replicates). Then the RSD of the averages was calculated and used to estimate the FRSD. In cases where the RSD was below 3\%, the FRSD was set to $3 \%$. This group used a slightly different comparison criterion. The upper limit was equal to Sample $1 \times(1+4 \times$ FRSD $)$ and the lower limit was Sample $1 \div(1+4 \times$ FRSD). If the mean of Sample 2 fell within the comparison interval, the samples were indistinguishable. Unlike the ASTM E2927 comparison criteria, the mean of Sample 1 is not at the center of the interval and the SD of the measurement is not used. However because of these differences, the end result, indistinguishable or distinguished, is the same regardless of which sample was selected as Sample 1.

Both approaches of determining a minimum FRSD were applied to the FIU M\&M database as well as other comparison criteria. The calculated FRSD differed greatly for some elements based on which method was used, Table 15 . For example, ${ }^{208} \mathrm{~Pb}$ ranged from 3.8 to 12.2. To evaluate each criteria, 52 duplicate samples which were run anywhere from 3-8 months apart, Table 16. The duplicate samples were analyzed using 
the same instrumental parameters as the M\&M glass samples with 3 fragments with 5 replicates each.

The comparison criterion used to compare samples in the database was the comparison interval mentioned above in which the upper limit was Sample $1 \times(1+4 \times$ FRSD) and the lower limit was Sample $1 \div(1+4 \times$ FRSD). This comparison interval was selected because the end result, indistinguishable or distinguished, is the same regardless of which sample was selected as Sample 1. The results using the duplicates and the different FRSDs can be found in Table 17. 
Table 15 - Shown are the calculated \% RSDs

\begin{tabular}{|c|c|c|c|c|c|c|c|c|c|c|c|c|c|c|c|c|}
\hline & ${ }^{7} \mathbf{L i}$ & ${ }^{25} \mathrm{Mg}$ & ${ }^{27} \mathbf{A l}$ & ${ }^{39} \mathbf{K}$ & ${ }^{42} \mathbf{C a}$ & ${ }^{49} \mathrm{Ti}$ & ${ }^{55} \mathrm{Mn}$ & ${ }^{57} \mathrm{Fe}$ & ${ }^{85} \mathbf{R b}$ & ${ }^{88} \mathrm{Sr}$ & ${ }^{90} \mathrm{Zr}$ & ${ }^{137} \mathbf{B a}$ & ${ }^{139} \mathbf{L a}$ & ${ }^{140} \mathrm{Ce}$ & ${ }^{146} \mathrm{Nd}$ & ${ }^{208} \mathbf{P b}$ \\
\hline $\begin{array}{l}\text { NIST1831 } \\
\text { Inter-day Variation of Control }\end{array}$ & 4.5 & 3.0 & 3.2 & 3.0 & 3.0 & 3.0 & 3.0 & 3.0 & 3.7 & 7.5 & 7.1 & 5.1 & 5.8 & 5.2 & 5.6 & 12.2 \\
\hline $\begin{array}{l}\text { FGS1 } \\
\text { Inter-day Variation of Control }\end{array}$ & 3.7 & 3.0 & 3.7 & 3.0 & 3.0 & 3.2 & 3.0 & 3.0 & 3.0 & 3.0 & 4.6 & 3.0 & 3.9 & 7.8 & 3.6 & 9.1 \\
\hline Same Source NIST1831 & 6.8 & 3.0 & 3.0 & 3.0 & 3.0 & 3.0 & 3.0 & 3.0 & 3.0 & 3.0 & 3.0 & 3.0 & 3.0 & 3.0 & 5.6 & 3.8 \\
\hline Same Source FGS1 & 6.6 & 3.0 & 3.0 & 3.0 & 3.0 & 3.8 & 3.0 & 3.0 & 3.0 & 3.0 & 3.0 & 3.0 & 3.0 & 3.0 & 3.7 & 3.0 \\
\hline Different Source M\&M & 8.2 & 3.0 & 3.0 & 3.0 & 3.0 & 3.0 & 3.0 & 3.0 & 3.0 & 3.0 & 3.1 & 3.0 & 3.1 & 3.0 & 5.3 & 3.6 \\
\hline Max \%FRSD for M\&M & 8.2 & 3.0 & 3.7 & 3.0 & 3.0 & 3.8 & 3.0 & 3.0 & 3.7 & 7.5 & 7.1 & 5.1 & 5.8 & 7.8 & 5.6 & 12.2 \\
\hline Adjusted \%FRSD for M\&M & 8.2 & 3.0 & 7.0 & 3.0 & 4.0 & 3.8 & 3.0 & 3.0 & 4.0 & 4.0 & 7.1 & 5.1 & 5.8 & 9.0 & 7.0 & 12.2 \\
\hline
\end{tabular}


Table 16 - Duplicate samples and the days of analysis

\begin{tabular}{|c|c|c|}
\hline Sample & Date of Sample Analysis & Date of Duplicate Analysis \\
\hline 001 Outer & $10 / 3 / 16$ & $5 / 25 / 17$ \\
\hline 002 Inner & $10 / 3 / 16$ & $6 / 14 / 17$ \\
\hline 003 Inner & $10 / 3 / 16$ & $5 / 18 / 17$ \\
\hline 004 Inner & $10 / 3 / 16$ & $5 / 13 / 17$ \\
\hline 008 Inner & $10 / 13 / 16$ & $5 / 25 / 17$ \\
\hline 015 Inner & $10 / 14 / 16$ & $6 / 14 / 17$ \\
\hline 017 Inner & $10 / 14 / 16$ & $6 / 14 / 17$ \\
\hline 019 Outer & $10 / 14 / 16$ & $5 / 19 / 17$ \\
\hline 025 Inner & $10 / 15 / 16$ & $6 / 14 / 17$ \\
\hline 029 Outer & $10 / 17 / 16$ & $6 / 14 / 17$ \\
\hline 030 Outer & $10 / 17 / 16$ & $5 / 25 / 17$ \\
\hline 031 Inner & $10 / 17 / 16$ & $5 / 19 / 17$ \\
\hline 033 Outer & $10 / 17 / 16$ & $5 / 25 / 17$ \\
\hline 036 Inner & $10 / 20 / 16$ & $6 / 14 / 17$ \\
\hline 036 Outer & $10 / 20 / 16$ & $5 / 13 / 17$ \\
\hline 039 Inner & $10 / 20 / 16$ & $6 / 14 / 17$ \\
\hline 040 Outer & $10 / 20 / 16$ & $5 / 18 / 17$ \\
\hline 041 Inner & $10 / 20 / 16$ & $6 / 14 / 17$ \\
\hline 045 Outer & $10 / 22 / 16$ & $5 / 18 / 17$ \\
\hline 046 Inner & $10 / 22 / 16$ & $5 / 19 / 17$ \\
\hline 053 Inner & $11 / 3 / 16$ & $6 / 14 / 17$ \\
\hline 057 Outer & $11 / 4 / 17$ & $5 / 18 / 17$ \\
\hline 059 Outer & $11 / 4 / 17$ & $5 / 19 / 17$ \\
\hline 064 Outer & $11 / 5 / 16$ & $5 / 18 / 17$ \\
\hline 071 Outer & $11 / 6 / 16$ & $5 / 13 / 17$ \\
\hline 073 Inner & $11 / 7 / 16$ & $6 / 14 / 17$ \\
\hline 076 Inner & $11 / 7 / 16$ & $5 / 19 / 17$ \\
\hline 078 Outer & $11 / 7 / 16$ & $5 / 19 / 17$ \\
\hline 083 Inner & $11 / 8 / 16$ & $5 / 25 / 17$ \\
\hline 094 Inner & $1 / 5 / 17$ & $5 / 19 / 17$ \\
\hline 097 Inner & $1 / 6 / 17$ & $6 / 14 / 17$ \\
\hline 105 Outer & $1 / 12 / 17$ & $6 / 14 / 17$ \\
\hline 107 Inner & $1 / 12 / 17$ & $5 / 25 / 17$ \\
\hline 109 Inner & $1 / 12 / 17$ & $6 / 14 / 17$ \\
\hline 119 Outer & $1 / 18 / 17$ & $5 / 18 / 17$ \\
\hline 122 Outer & $1 / 24 / 17$ & $5 / 18 / 17$ \\
\hline 133 Inner & $1 / 26 / 17$ & $5 / 13 / 17$ \\
\hline 145 Outer & $1 / 29 / 17$ & $5 / 18 / 17$ \\
\hline 146 Inner & $1 / 29 / 17$ & $5 / 13 / 17$ \\
\hline 149 Outer & $2 / 1 / 17$ & $5 / 19 / 17$ \\
\hline 152 Outer & $2 / 1 / 17$ & $5 / 13 / 17$ \\
\hline 153 Inner & $2 / 1 / 17$ & $5 / 19 / 17$ \\
\hline 155 Inner & $2 / 1 / 17$ & $5 / 18 / 17$ \\
\hline 161 Outer & $2 / 2 / 17$ & $5 / 13 / 17$ \\
\hline 181 Outer & $2 / 8 / 17$ & $5 / 18 / 17$ \\
\hline 183 Outer & $2 / 8 / 17$ & $5 / 13 / 17$ \\
\hline 185 Outer & $2 / 8 / 17$ & $5 / 25 / 17$ \\
\hline 188 Inner & $2 / 8 / 17$ & $5 / 19 / 17$ \\
\hline 191 Outer & $2 / 9 / 17$ & $5 / 18 / 17$ \\
\hline 195 Inner & $2 / 11 / 17$ & $5 / 19 / 17$ \\
\hline 204 Inner & $2 / 16 / 17$ & $5 / 19 / 17$ \\
\hline 210 Outer & $2 / 17 / 17$ & $5 / 25 / 17$ \\
\hline
\end{tabular}


Table 17 - The results for the duplicate samples with different comparison criteria. *This is the result of the ASTM E2927 comparison criterion. This criterion depends on which sample is selected as the Known sample, so the original data in the database was selected as the duplicate. The FRSD selected for further use in this study was FRSD (Adjusted FIU).

\begin{tabular}{|l|c|}
\hline \multicolumn{1}{|c|}{ Comparison Criteria } & Duplicates \\
Sample 1 mean $\times(1+4 \times$ FRSD) & Type I Error \\
Samplean $\div(1+4 \times$ FRSD) & $(\%)$ \\
\hline *ASTM 2927: \pm 4s (FRSD 3\%) (Known=Original) & 52 \\
FRSD 3\% & 58 \\
FRSD 4\% & 40 \\
FRSD 5\% & 27 \\
FRSD 3\% Limited Menu (No Al, Zr, Ce, Pb ) & 31 \\
FRSD (FIU FGS01) & 37 \\
FRSD (FIU NIST1831) & 44 \\
FRSD (Max Control Glass) & 33 \\
FRSD (FIU Same Source/ Different Source) & 56 \\
FRSD (Max FIU) & 31 \\
FRSD (Adjusted FIU) & 2 \\
\hline
\end{tabular}

Even after determining the maximum inter-day variation of the M\&M database, some elements were still distinguishing the duplicate pairs, Figure 38. The issues with Al and $\mathrm{Ca}$ appeared to be caused by the calibration of the pulse/analog mode of the detector. For some of the samples that were distinguished by these particular elements, the counts were above 2 million, which also indicates the problem is caused by the pulse/analog calibration. 


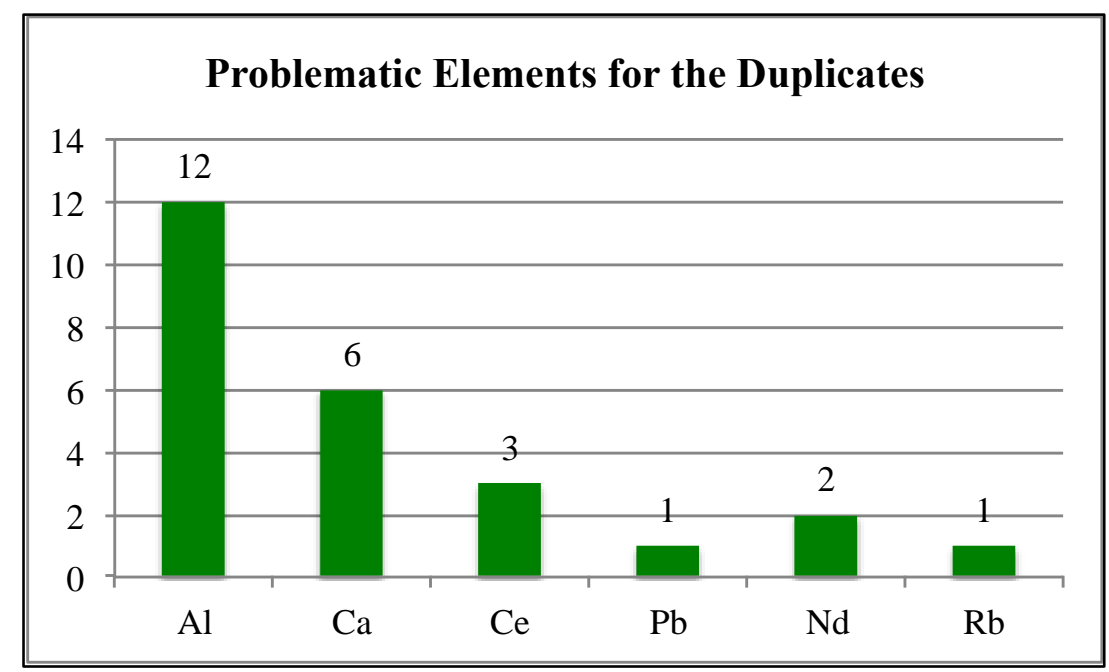

Figure 38 - Elements distinguishing duplicates using the max FIU FRSD

The duplicates that were distinguished by $\mathrm{Nd}$ and $\mathrm{Rb}$ were caused by fall off the cliff effects where the duplicate mean fell just outside the comparison interval, Figure 39. This could be attributed to the low concentration of these elements in the duplicates $(<2$ ppm). The FRSD for all of these elements ( $\mathrm{Al}, \mathrm{Ca}, \mathrm{Nd}, \mathrm{Rb})$ was increased, which helped correctly associate the duplicates. 
Database Sample Average

Database Sample Average $\times(1+4 \times$ FRSD $)$ - - - - - - -

Database Sample Average $\div(1+4 \times$ FRSD $)$

Duplicate Mean
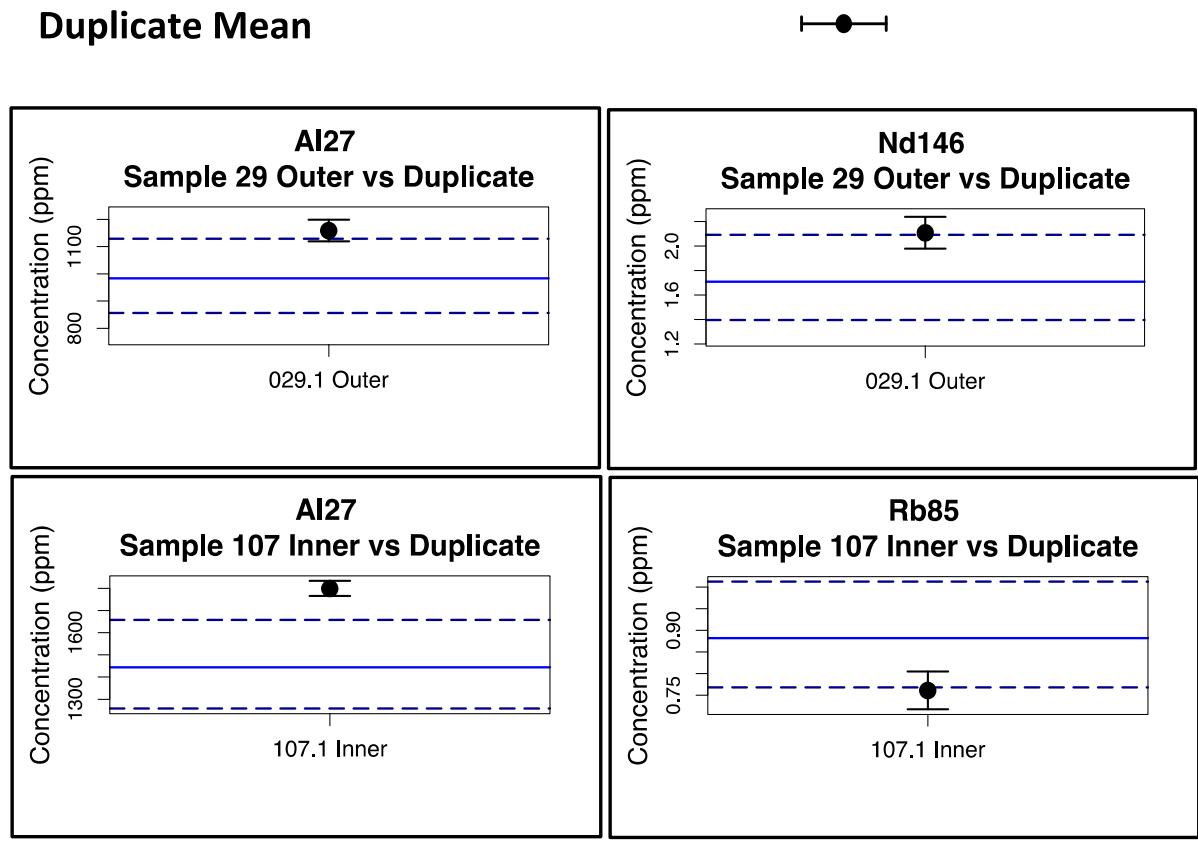

Figure 39 - Examples of some duplicate pairs being distinguished using the maximum FRSDs of the M\&M database.

Increasing the FRSDs for $\mathrm{Ce}$ and $\mathrm{Pb}$ did not alleviate the problem for three of the comparison pairs. The problem with the Ce pairs is caused by the calibration of the pulse/analog mode of the detector. Both samples that were distinguished from their duplicate had an unusually high (> $2000 \mathrm{ppm}$ ) Ce concentration compared to most samples in the database. There are only 7 samples out of 333 that have a Ce concentration greater than $2000 \mathrm{ppm}$. The transient signal for Ce in these samples was above 2 million counts, which is the point where the detector switches modes from pulse to analog. Improper calibration of the detector caused the concentration of Ce to be $7900 \pm 26 \mathrm{ppm}$ for the original and $4520 \pm 69 \mathrm{ppm}$ for the duplicate sample for one pair and $2110 \pm 17$ ppm for the original and $3680 \pm 56 \mathrm{ppm}$ for the duplicate in the other distinguished pair. 
Therefore, Ce was flagged in the 7 samples with a concentration greater than $2000 \mathrm{ppm}$ and not used in comparisons involving these samples.

$\mathrm{Pb}$ has been a consistent problem. Over half of the samples in this database contain a concentration of $\mathrm{Pb}$ around $2 \mathrm{ppm}$. Differences in the performance of the laser and ICP-MS between days could result in a spiky, uneven signal. A look at the first replicate measurement of each fragment for the comparison pair that was distinguished by $\mathrm{Pb}$, Figure 40, showed that some replicates in the original (black) had spikes. Even after filtering with GLITTER, these spikes caused the concentration of ${ }^{208} \mathrm{~Pb}$ to range from 3.1-6.7 ppm in the original sample. Since the comparison criterion used does not take the SD of the measurement into consideration, the poor reproducibility of this sample was not accounted for. In the future $\mathrm{Pb}$ may be excluded from the database, but for this study it was kept.

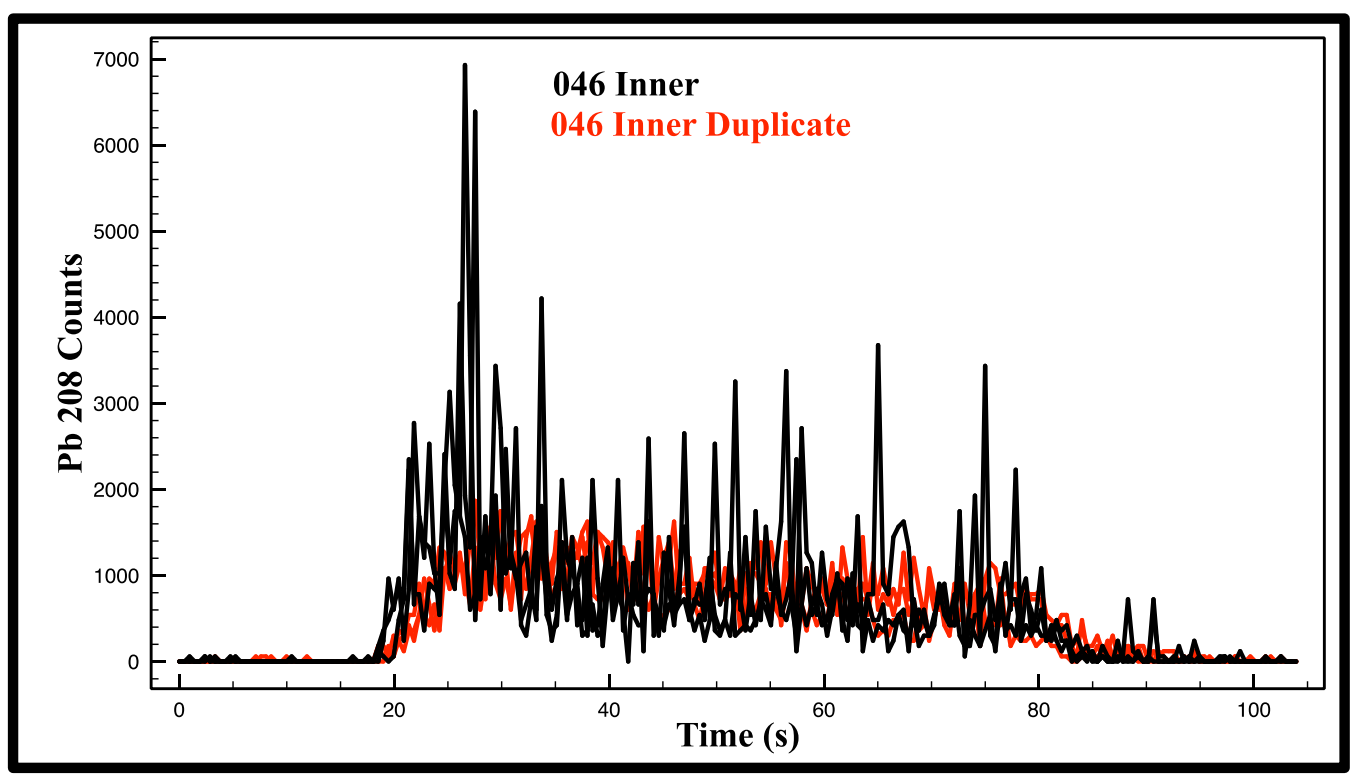

Figure 40 - Transient signal for $\mathrm{Pb} 208$ in duplicate pair

The FRSD determined for $\mathrm{Sr}$ when examining the inter-day variation of the NIST1831 control glass very large (7.5\%) especially when compared to the FRSDs determined for 
this element using FGS1 (3\%) and the other approaches. This large variation in Sr could not be determined. Therefore, the FRSD was reduced to $4 \%$ for future analyses. This new FRSD for the M\&M database is denoted as "Adjusted" in Table 15 and Table 17. After removing $\mathrm{Ce}$ from the problematic samples, this comparison criterion resulted in a $2 \%$ Type I error. This comparison criterion will be used from now on. The daily control glasses NIST1831 and FGS1 were also evaluated using the this comparison criteria. However, these samples only have 5 replicates. The daily control glasses were analyzed 40 (FGS1) or 42 (NIST1831) times over the course of 8 months (10/3/16 - 6/14/17). Using the adjusted FRSD, the results of the control glass FGS1 was correctly associated with itself $96 \%$ of the time and NIST1831 was correctly associated $78 \%$ of the time. Figure 41, shows the problematic elements. As can be seen, most of the inter-day measurements were distinguished by $\mathrm{Pb}$ and $\mathrm{Sr}$. The differences in $\mathrm{Pb}$ for NIST1831 were caused by only two days of analysis, November $4^{\text {th }}$ and November $6^{\text {th }}$ being distinguish from most of the other days. Examining the daily control charts for NIST1831, Figure 36, it can be observed that these two days have a higher concentration of $\mathrm{Pb}$ than the others. A similar thing happened with FGS1, but for a different day, October $22^{\text {nd }}$. The control chart for FGS1 can be found in the Appendix. When these problematic days are removed, the correct association rates are 100\% for FGS1 and 84\% for NIST1831.

The poor performance of $\mathrm{Sr}$ in NIST1831 cannot be explained. Unlike $\mathrm{Pb}$, it is not the result of a single day of analysis. The concentration of Sr in NIST1831 is a little more than FGS1, 87 and 55 ppm respectively, Table 14. Since Sr performs fine in FGS1, it is not an issue with limits of detection. It is therefore hypothesized to be an issue with the 
NIST1831 glass. This glass is clear, colorless, and much thicker than FGS1. Its larger size could be causing issues with particles being successfully carried to the ICP-MS or the issue could be with the laser sample interaction. The FGS glasses are cloudier than the NIST1831 glass, thus it is easy to focus the laser on the surface of the FGS glasses. However, due to the transparency of the NIST1831 glass, care had to be taken to ensure that the laser was properly focused on the top of the glass and not the bottom. These elements can be removed in future studies, but since the duplicate glass samples did not have a problem with Sr this element was kept in the element menu.

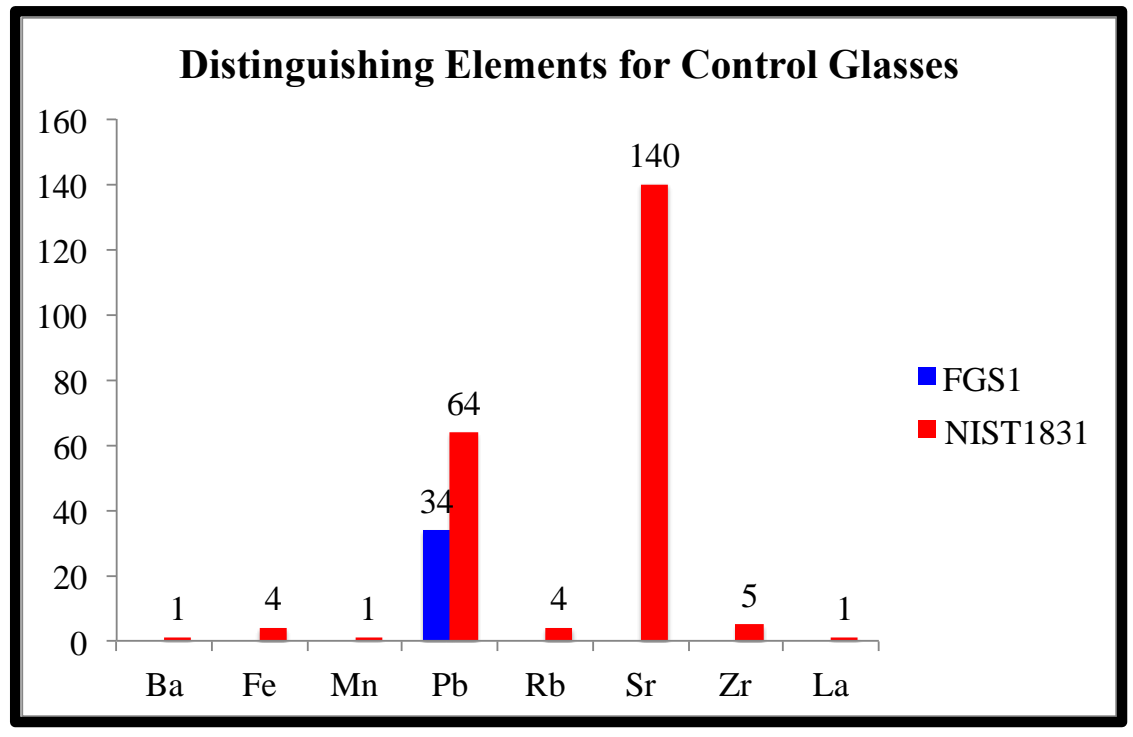

Figure 41 - Using the selected FRSD, the elements shown in the graph were found to incorrectly distinguish inter-day control samples. The $\mathrm{Sr}$ variation seen in NIST1831 was only seen in this glass. FGS1 and the duplicates appeared to have no problem with $\mathrm{Sr}$. $\mathrm{Pb}$ reproducibility was an issue for both control glasses and some of the duplicate pairs.

\subsubsection{Blind Study}

To further test the accuracy of the match criterion selected in 7.7 , another student was asked to randomly select ten glass fragments from the M\&M collection set and place 
them in pill boxes labeled as Blind \#1-10. These glass samples were analyzed using the same instrument parameters as the M\&M collection.

Each blind sample was compared to the database using the match criterion determined in the previous section. Table 18 shows the results of the comparison. All blind samples were associated with their duplicate in the database. Some of the blind samples had more than one database match. In these cases, the other samples were usually from a vehicle of the same make. Automobile manufacturers have an annual contract with glass manufacturers. Therefore, vehicles of the same make and year are highly likely to have the same glass manufacturer. This can be observed in the blind study. One exception is Blind 6. This sample was indistinguishable with two Hondas and one Dodge. The window manufacturer information was only available for sample 210 . A Pittsburgh Glass Works division called Mopar manufactured this glass. An Internet search on the Mopar website showed that Mopar glass is mostly found on Dodge, Chrysler, Jeep vehicles, but is also offered as a replacement glass. Since the other two database hits are Hondas, it could be possible that these vehicles had a Mopar replacement windshield. It is possible that the windowpanes were produced by the same manufacturer. 


\begin{tabular}{|c|c|c|c|c|c|}
\hline Sample & Database Hits & Year & Make & Model & True Identity of Blind \\
\hline Blind 1 & $\begin{array}{l}\text { 029.1 Inner \& Outer } \\
\text { 053.3 Inner }\end{array}$ & $\begin{array}{l}2016 \\
2016\end{array}$ & $\begin{array}{l}\text { Toyota } \\
\text { Toyota }\end{array}$ & $\begin{array}{l}\text { Tundra } \\
\text { Tundra }\end{array}$ & 053.3 Inner \\
\hline Blind 2 & $\begin{array}{l}\text { 029.1 Inner \& Outer } \\
053.3 \text { Inner }\end{array}$ & $\begin{array}{l}2016 \\
2016\end{array}$ & $\begin{array}{l}\text { Toyota } \\
\text { Toyota }\end{array}$ & $\begin{array}{l}\text { Tundra } \\
\text { Tundra }\end{array}$ & 029.1 Outer \\
\hline Blind 3 & 015.1 Inner \& Outer & 2010 & Toyota & Tacoma & 015.1 Outer \\
\hline Blind 4 & 017.1 Inner \& Outer & 2011 & Scion & $\mathrm{tC}$ & 017.1 Outer \\
\hline Blind 5 & 039.1 Inner & 2016 & Mazda & $\mathrm{CX}-3$ & 039.1 Inner \\
\hline Blind 6 & $\begin{array}{l}\text { 069.1 Outer } \\
\text { 073.1 Inner \& Outer } \\
\text { 210.1 Inner \& Outer }\end{array}$ & $\begin{array}{l}2013 \\
2015 \\
2016\end{array}$ & $\begin{array}{l}\text { Honda } \\
\text { Honda } \\
\text { Dodge }\end{array}$ & $\begin{array}{l}\text { Civic } \\
\text { CR-Z } \\
\text { Challenger }\end{array}$ & 073.1 Inner \\
\hline Blind 7 & $\begin{array}{l}025.1 \text { Inner } \\
041.1 \text { Inner } \\
\text { 097.1 Inner }\end{array}$ & $\begin{array}{l}2010 \\
2010 \\
2010\end{array}$ & $\begin{array}{l}\text { Kia } \\
\text { Kia } \\
\text { Kia }\end{array}$ & $\begin{array}{l}\text { Forte } \\
\text { Forte } \\
\text { Forte }\end{array}$ & 097.1 Inner \\
\hline Blind 8 & $\begin{array}{l}\text { 002.1 Inner \& Outer } \\
\text { 069.1 Outer }\end{array}$ & $\begin{array}{l}2013 \\
2013\end{array}$ & $\begin{array}{l}\text { Honda } \\
\text { Honda }\end{array}$ & $\begin{array}{l}\text { Civic } \\
\text { Civic }\end{array}$ & 069.1 Outer \\
\hline Blind 9 & 105.1 Inner \& Outer & 2014 & Mitsubishi & Outlander & 105.1 Outer \\
\hline Blind 10 & 109.1 Inner \& Outer & 2008 & Mitsubishi & Lancer & 109.1 Inner \\
\hline
\end{tabular}

Table 18 - Comparing the blind samples to the database

\subsection{Discrimination Potential}

Using the comparison criteria from the previous section, the samples in the M\&M database were compared to each other resulting in 55278 comparison pairs. The elements distinguishing each comparison pair were examined to determine the percent discrimination of each element. Comparison pairs that were distinguished by only one 
element were also examined to determine if the pairs shared the same source, make, and/or year. The results are shown in Figure 42. $\mathrm{Ca}$ and $\mathrm{Mg}$ were the least discriminating elements with only $14.9 \%$ and $41.1 \%$ of the comparison pairs being distinguished by them, respectively. The low discrimination potential for these elements can be explained because this database contains only soda-lime vehicle glass, so the composition of main ingredients and modifiers are expected to be somewhat controlled within each glass manufacturer. However elements such as $\mathrm{Fe}$ and $\mathrm{Sr}$, that are common contaminants in glass, have a high discrimination potential. It also isn't surprising to see that some similar vehicles are only discriminated by these elements. This could be because the concentration of these contaminants changed over time within a manufacturer so glass produced on different days can be distinguished. 


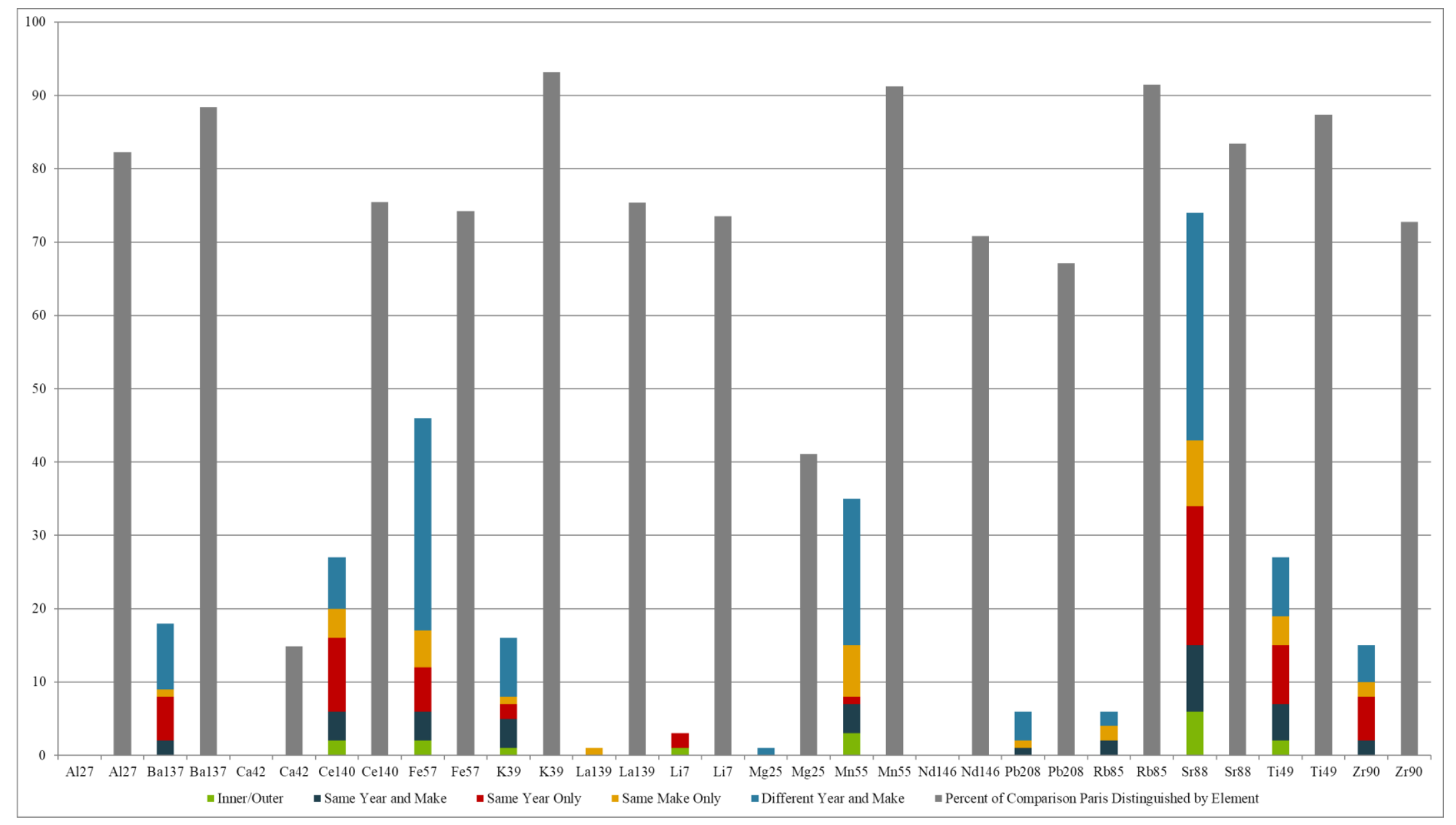

Figure 42 - Discrimination potential of each element. The grey columns represent the percent $(\%)$ of comparisons that were distinguished by that element. The colored segmented columns represent how many comparisons were only distinguished by that element and what type of samples they were (same year, same make etc.) 


\section{CHAPTER 8. Collection and Evaluation of Glass Databases}

\subsection{Glass Databases}

A survey was sent out to forensic laboratories across the world asking if they had a glass database generated by (LA) ICP-MS that could be shared with the interpretation working group. If possible the databases were reduced to only soda-lime glass. However, some of the databases contained casework samples in which the true source of the glass is unknown. Table 19 shows a brief description of each database and how many samples it contained after removing non-soda-lime glass.

\subsection{Random Match Probability}

The random match probability can be estimated as the false inclusion rate of the database. That is, the number of comparison pairs of samples that originated from different sources that were found to be indistinguishable. Each database in Table 19 was used to calculate a random match probability to see what effect sample size and element menu has. For the comparisons, each database was limited to only elements listed within ASTM E2927-16e1 without Hf since it is found to be highly correlated with $\mathrm{Zr}$ in glass, Figure $37:{ }^{7} \mathrm{Li},{ }^{25} \mathrm{Mg},{ }^{27} \mathrm{Al},{ }^{39} \mathrm{~K},{ }^{42} \mathrm{Ca},{ }^{57} \mathrm{Fe},{ }^{49} \mathrm{Ti},{ }^{55} \mathrm{Mn},{ }^{85} \mathrm{Rb},{ }^{88} \mathrm{Sr},{ }^{90} \mathrm{Zr},{ }^{137} \mathrm{Ba},{ }^{139} \mathrm{La},{ }^{140} \mathrm{Ce}$, ${ }^{146} \mathrm{Nd},{ }^{208} \mathrm{~Pb}$. For databases that do not contain all of these elements, the error rate was calculated using their limited menu.

The comparison criterion described in the previous section was used to compare the samples within each database even though this criterion was designed to be used with the M\&M glass database. Ideally each lab should determine their inter-day variation and if databases are to be combined a comparison criterion should be used that 
encompasses all inter-day and inter-lab variations. Table 20 shows the false inclusion rate for each database. The results in this table are consistent with the two previous reports in the literature that the false inclusion error rate is approximately $0.1 \%$ for the databases examined. With the exception of Database 4 which is only contains 43 samples, the random match probability was found to be $\sim 0.1 \%$ for the collected databases. Each database is a snapshot of a specific place and time, but regardless of where the database came from the random match probability was similar. Database 2 is the FIU M\&M vehicle database that contains many vehicles of the same make and year and thus has a higher error rate but when the vehicles known to be related (same make and year) are excluded from the number of indistinguishable pairs, the error rate is $0.13 \%$. Since the FIU M\&M database also has thickness measurements, these were used to further compare the pairs with indistinguishable elemental profiles. Of the 132 pairs, 26 can be distinguished by thickness measurements. The samples that were distinguished by thickness did not follow any visible pattern. There were some vehicles that were the same year, make, and model (6 pairs); some pairs were inner and outer panes from the same vehicle ( 3 pairs); some were different years and/or different makes (17 pairs).

Interestingly, even though it had a limited element menu, Database 6 also had a false inclusion error rate of $\sim 0.1 \%$. This shows that not all the elements listed in ASTM E-2927 are necessary to discriminate glass samples. It may be possible to limit the element menu, which will allow for more databases to be combined if combining is deemed appropriate. 
Table 19 - Description of each database donated to the FIU GIWG

\begin{tabular}{|c|c|c|c|c|c|c|}
\hline & Database 1 & Database 2 & Database 3 & Database 4 & Database 5 & Database 6 \\
\hline Instrument & LA-ICP-MS & LA-ICP-MS & ICP-MS & LA-ICP-MS & LA-ICP-MS & LA-ICP-MS \\
\hline \# of Samples & 517 & 333 & 300 & 43 & 300 & 969 \\
\hline $\begin{array}{l}\text { Replicates per } \\
\text { Sample }\end{array}$ & 9 & 15 & 3 & 5 & 6 & 5 \\
\hline 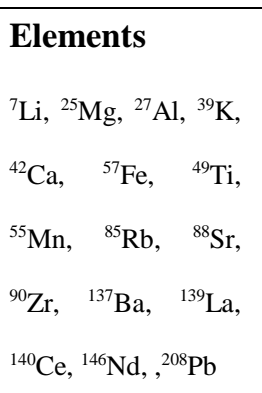 & $\begin{array}{c}\text { Additional Elements: } \\
{ }^{59} \mathrm{Co},{ }^{89} \mathrm{Y},{ }^{95} \mathrm{Mo},{ }^{121} \mathrm{Sb} \\
{ }^{182} \mathrm{~W},{ }^{232} \mathrm{Th},{ }^{238} \mathrm{U}\end{array}$ & --- & $\begin{array}{c}\text { Elements not Used: } \\
{ }^{7} \mathrm{Li},{ }^{27} \mathrm{Al},{ }^{39} \mathrm{~K},{ }^{42} \mathrm{Ca},{ }^{57} \mathrm{Fe}, \\
{ }^{146} \mathrm{Nd}\end{array}$ & --- & $\begin{array}{l}\text { Additional } \\
\text { Elements: }{ }^{23} \mathrm{Na}\end{array}$ & $\begin{array}{c}\text { Elements not Used: } \\
{ }^{7} \mathrm{Li},{ }^{25} \mathrm{Mg},{ }^{27} \mathrm{Al},{ }^{42} \mathrm{Ca},{ }^{57} \mathrm{Fe}, \\
{ }^{146} \mathrm{Nd}\end{array}$ \\
\hline $\begin{array}{l}\text { Types of } \\
\text { Samples }\end{array}$ & $\begin{array}{c}\text { Casework \& Survey: } \\
\text { Float Glass }\end{array}$ & $\begin{array}{c}\text { Survey: } \\
\text { Float Glass }\end{array}$ & $\begin{array}{c}\text { Survey: } \\
\text { Float Glass \& } \\
\text { Containers }\end{array}$ & Casework & Casework & Casework \\
\hline Calibration & External Calibration & $\begin{array}{l}\text { Single Point } \\
\text { FGS2 }\end{array}$ & $\begin{array}{c}\text { External } \\
\text { Calibration }\end{array}$ & $\begin{array}{l}\text { Single Point } \\
\text { NIST612 }\end{array}$ & Single Point FGS2 & $\begin{array}{l}\text { Single Point } \\
\text { NIST612 }\end{array}$ \\
\hline
\end{tabular}


Table 20 - False inclusion rates for the donated databases

\begin{tabular}{|lcccc|}
\hline & Samples & Comparison Pairs & Indistinguishable Pairs & Error (\%) \\
\hline Database 1 & 517 & 133386 & 183 & 0.14 \\
Database 2 & 333 & 55278 & 132 & 0.24 \\
Database 2 & 333 & 55219 & 73 & 0.13 \\
Without Related & & 44850 & 53 & 0.12 \\
Database 3 & 300 & 903 & 0 & 0 \\
Database 4 & 43 & 68265 & 44 & 0.06 \\
Database 5 & 370 & 468996 & 557 & 0.12 \\
Database 6 & 969 & & & \\
\hline
\end{tabular}

Each LA-ICP-MS database was donated by a participant of the GIWG first inter-

laboratory study that will be discussed in a later chapter. If each participant used the same LA-ICP-MS method that they used in the first inter-laboratory study, the data for the samples can be evaluated to see what elements have similar concentration for each lab regardless of what calibration technique was used. This could help in eliminating problematic elements. Each lab's data for the K samples compared to each other and to the $M \& M$ database sample as well as their data for the quality control glasses can be found in the Appendix. 


\section{CHAPTER 9. Equivalence Test vs. ASTM E2927 Match Criterion}

The EAWG evaluated several statistical tests to determine which one produced the lowest false inclusion and false exclusion error rates. One test that has not yet been assessed as a possible match criterion for forensic glass comparisons is the equivalence test also known as the two one sided t-tests (TOST). This test is another type of hypothesis testing similar to the T-test. However, unlike the T-test, the equivalence test evaluates the null hypothesis that the sample means are different, $H_{0}: \mu_{1} \neq \mu_{2}$. In the criminal justice system, you are innocent until proven guilty. One statistician is pushing for the equivalence test to be used more in forensic comparisons, since the null hypothesis is that the sample from the suspect did not come from the scene of the crime.

\subsection{Basics of the Equivalence Test}

For this test, the analyst defines an acceptance criterion $(\theta)$ on the basis of prior knowledge of the measurement as well as its intended application. The acceptance criterion is the maximum value that two sample means can differ by and still be deemed practically equivalent. For glass analysis, an acceptance criterion has to be assigned for each element that is being compared. If the $90 \%$ confidence interval of the difference in the elemental means of the $\mathrm{K}$ and $\mathrm{Q}$ samples falls within $[-\theta, \theta]$, the null hypothesis is rejected. The samples are deemed "practically equivalent". However, if one elemental comparison falls outside $[-\theta, \theta]$, the samples are considered distinguishable. Figure 43 shows an example of sample means that are practically equivalent, $\mathrm{A}$ and $\mathrm{C}$, and sample means that are distinguishable, $\mathrm{B}$. 


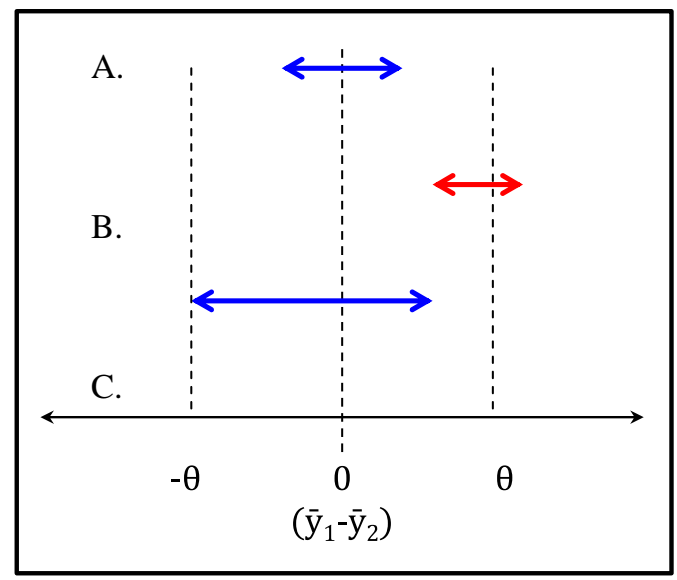

\section{Figure 43 - Modified from [61], this figure depicts how the equivalence test decides if the sample means are practically equivalent.}

The application of the equivalence test to elemental data is not very straightforward; the most challenging part is defining the acceptance criteria $[-\theta, \theta]$. Some papers and statisticians suggest using a database to define $\theta$; so for this study, the variation within a subset of 109 float architectural glass samples from known different sources was used to determine $\theta$ for each element as well as a subset of 63 glass samples produced at the same manufacturing plant over the course of a couple of years (19972001). However, not all forensic analysts have access to a database. Given the lack of a suitable database, the 9 replicate measurements on the known sample were also used to define $\theta$. One publication was found within the analytical chemistry literature that showed how to calculate $\theta$, and it was applied to these calculations [61].

Equation (18) was used to ensure that the standard deviation represents true measurement precision. In this equation, $\gamma$ was selected to be $20 \%$. The $\chi_{(\gamma, n-1)}^{2}$ was solved for using CHIINV (1- $\gamma, \mathrm{n}-1)$ in Excel. The $n$ for this calculation was either 109 or 63 if $\theta$ was being calculated using a database or 9 if $\theta$ was being calculated using only the replicate measurements done on the known sample. The $s$ stands for the standard 
deviation of an element within the database or the standard deviation of the element within the replicate measurements of the known. This calculation calculates a confidence limit for the standard deviation.

$$
s^{*}=s \sqrt{\frac{n-1}{\chi_{(\gamma, n-1)}^{2}}}
$$

Equation (19) was used to determine $\theta$ for each element. As previously stated, $\theta$ was calculated three ways. Two approaches used a database. The third approach used the replicate measurements of the $\mathrm{K}$ glass sample. Regardless of the approach, an $\alpha=\beta=0.05$ and $\delta=0$ was used to solve Equation (19).

$$
\theta=\delta+s^{*}\left[t_{(1-\alpha, 2 n-2)}+t_{(1-\beta / 2,2 n-2)}\right] \sqrt{2 / n}
$$

Equation (19)

The $90 \%$ confidence interval for the difference between the K and Q sample's mean was calculated using Equation (20) for each element. In this equation, $s_{p}^{2}$ stands for the pooled standard deviation of the $\mathrm{K}$ and Q samples, $\bar{y}_{1}-\bar{y}_{2}$ is the difference in means, and $n_{1}$ and $n_{2}$ are the number of replicate measurements for the $\mathrm{K}$ and $\mathrm{Q}$ samples.

$$
\left(\bar{y}_{1}-\bar{y}_{2}\right) \pm t_{\left(1-\alpha, n_{1}+n_{2}-2\right)} \sqrt{s_{p}^{2}\left(\frac{1}{n_{1}}+\frac{1}{n_{2}}\right)}
$$

The equivalence test was evaluated using LA-ICP-MS and ICP-MS data acquired during the Elemental Analysis Working Group inter-laboratory studies and by using the FIU ICP-MS database of 109 different known source float architectural glass samples. 


\subsection{Evaluating the Equivalence Test using EAWG Inter-Laboratory Studies}

The first inter-laboratory test was used to establish analytical protocols for LAICP-MS, ICP-MS, and $\mu \mathrm{XRF}$ and was not used to evaluate the equivalence test. In the second, third, and fourth inter-laboratory tests, the EAWG was asked to analyze float architectural glass samples that were labeled as known (K) and questioned (Q) samples. Table 21 shows a description of the samples and the number of labs using ICP-MS who participated in each test. These tests will be discussed in more detail in the following sections.

The data generated by each of the labs using ICP-MS was collected and used to evaluate the equivalence test. The results from the EAWG as well as the equivalence test are shown in Table 22. This table is modified from the EAWG publication [44]. For Test 2, the false inclusion and false exclusion rates were both calculated out of 19 comparisons from 7 laboratories. The design of the third inter-laboratory test did not account for false exclusion errors. The false inclusions for Test 3 were calculated from 126 comparisons from 7 laboratories. The false exclusions for Test 4 were calculated from 120 comparisons from 10 laboratories and the false inclusions were calculated out of 60 comparisons from 10 laboratories. 


\begin{tabular}{|c|c|c|c|c|c|c|c|c|c|}
\hline Test & \multicolumn{2}{|c|}{ Test 2} & \multicolumn{5}{|c|}{ Test 3} & \multicolumn{2}{|c|}{ Test 4} \\
\hline Sample & K1, Q1 & Q2 & K1 & K2 & Q1 & Q2 & Q3 & $\begin{array}{l}\text { K1, K2, } \\
\text { Q2, Q3 }\end{array}$ & Q1 \\
\hline Plant & Cardinal & Cardinal & Cardinal & Cardinal & Cardinal & Cardinal & Cardinal & Pilkington & Pilkington \\
\hline Manufacture Date & $4 / 1 / 01$ & $8 / 12 / 98$ & $8 / 17 / 01$ & $4 / 15 / 98$ & $8 / 31 / 01$ & $5 / 17 / 98$ & 7/17/98 & $3 / 3 / 10$ & $2 / 18 / 10$ \\
\hline Labs Participating & \multicolumn{2}{|c|}{7} & \multicolumn{5}{|c|}{7} & \multicolumn{2}{|c|}{10} \\
\hline
\end{tabular}

Table 21 - The samples used and the number of labs performing ICP-MS in EAWG 
Table 22 - False inclusion and exclusion error rates for the different statistical test using the EAWG inter-laboratory test data. The first four tests listed in this table are the reported results found in [44]. The three equivalence tests are the results from this study.

\begin{tabular}{|c|c|c|c|c|c|c|}
\hline \multirow{2}{*}{ Match Criteria } & \multicolumn{3}{|c|}{ False Inclusion Error Rate (\%) } & \multicolumn{3}{|c|}{ False Exclusion Error Rate (\%) } \\
\hline & Test 2 & Test 3 & Test $4^{c}$ & Test $2^{d}$ & Test $3^{\mathrm{e}}$ & Test $4^{\mathrm{f}}$ \\
\hline & 42 & - & 81 & 0 & 0 & 0 \\
\hline T-Test (0.05) & 74 & - & 93 & 0 & 1 & 0 \\
\hline T-Test (0.01) & 53 & - & 84 & 0 & 1 & 0 \\
\hline $\begin{array}{l}\text { T-Test with Bonferroni } \\
\text { Correction }\end{array}$ & 53 & - & 69 & 0 & 2 & 0 \\
\hline $\begin{array}{l} \pm 4 \text { Standard Deviations } \\
(\text { Standard Deviation }>3 \%)\end{array}$ & 0 & - & 28 & 0 & 5 & 0 \\
\hline Equivalence Test & & & & & & \\
\hline $\begin{array}{l}\text { ( } \theta \text { calculated from Subset } \\
\text { of FIU Database) }\end{array}$ & 100 & - & 80 & 0 & 5 & 0 \\
\hline $\begin{array}{l}\text { Equivalence Test } \\
(\theta \text { calculated from }\end{array}$ & & & & & & \\
\hline $\begin{array}{l}\text { Variation within } \\
\text { Manufacturing Plant) }\end{array}$ & 37 & - & 90 & 0 & 12 & 0 \\
\hline Equivalence Test & & & & & & \\
\hline ( $\theta$ calculated from Known) & 74 & - & 100 & 0 & 1 & 0 \\
\hline
\end{tabular}

As previously discussed in section 5.7, the match criterion that produced the lowest false inclusion and false exclusion rates was the comparison interval generated from the $\mathrm{K}$ sample with a range calculated for each element as the mean \pm 4 standard deviations with a minimum standard deviation equal to at least $3 \%$ of the mean value. 


\subsubsection{False Exclusions}

The false exclusions were calculated using the outcome of the second (K1 vs Q1) and fourth (K1 vs Q1.1, Q1.2, Q1.3, Q2.1, Q2.2, Q2.3 and K2 vs Q1.1, Q1.2, Q1.3, Q2.1, Q2.2, Q2.3) inter-laboratory studies. Please note that for the second test the participants were told to group the analyses from each question fragment together, but in the fourth test they were told not to group the question fragments of Q1 together. The fourth test better mimics a real forensic case, because one should not assume that all the glass fragments found on the suspect originated from the same source.

The results of the second and fourth inter-laboratory studies were interesting. The equivalence test resulted in a high false exclusion rate $(>70 \%)$, except when $\theta$ was calculated using the Cardinal manufacturing plant. When this $\theta$ was used, the second inter-laboratory study had an error rate of $37 \%$. This decrease in the error could be due to the fact that the second test contained Cardinal glass samples. However, the fourth test contained Pilkington samples, which the equivalence test failed to correctly associate with a $90 \%$ error rate.

When $\theta$, was calculated using the 9 measurements done on the $\mathrm{K}$, the equivalence test had a $100 \%$ false exclusion rate for the fourth study and $74 \%$ for the second. The acceptance criterion differed for each laboratory doing the analysis. ${ }^{88} \mathrm{Sr}$ for example had a range of $\theta$ s from $0.67-9.66 \mathrm{ppm}$. Figure 44 shows the equivalence test being used by each lab to evaluate the ${ }^{88} \mathrm{Sr}$ composition in the K1 vs Q2.1 comparison pair of the Pilkington glass samples. Though these samples originated from the same pane, seven of the ten labs found that the ${ }^{88} \mathrm{Sr}$ composition in the two was significantly different. ${ }^{88} \mathrm{Sr}$ 
was not the only distinguishable element, out of the total 120 comparison pairs used to calculate the false exclusion rate, 118 were distinguishable by two or more elements.
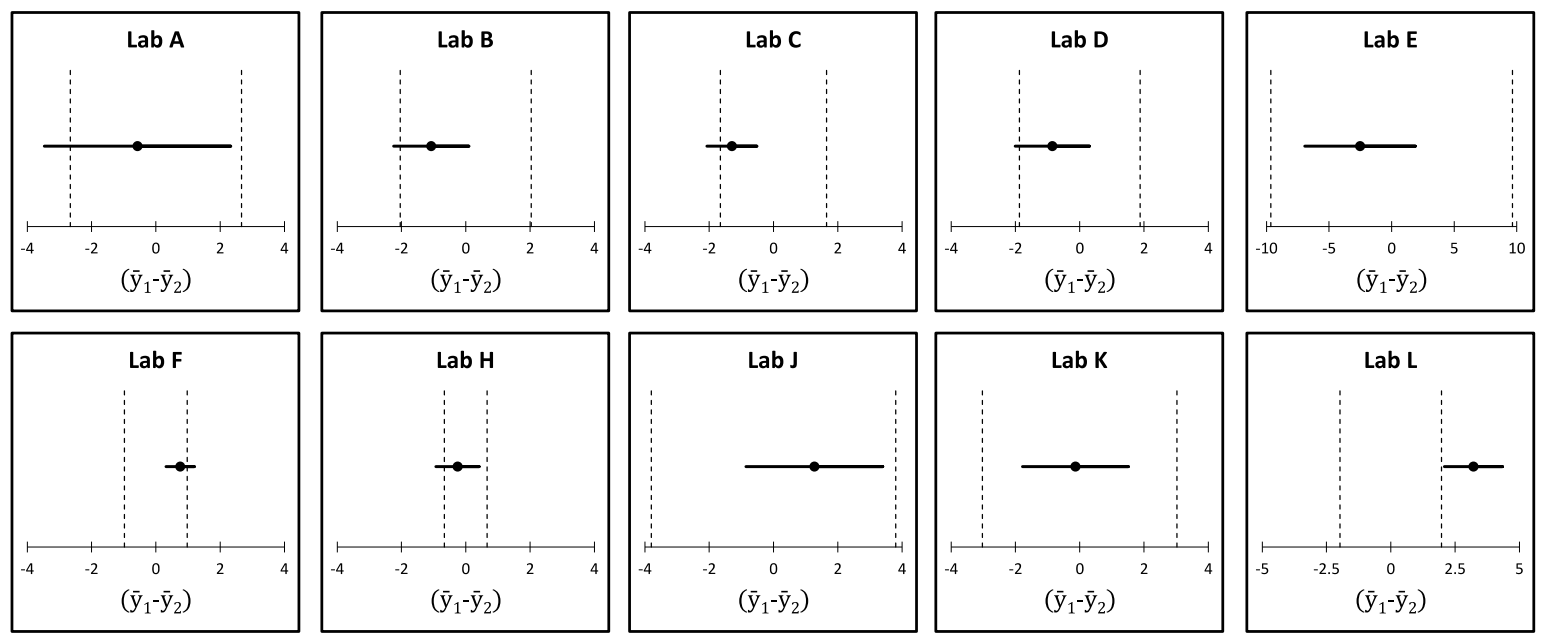

Figure 44 - Equivalence test for Sr using the 4th inter-laboratory data (K1 vs Q2.1)

\subsubsection{False Inclusions}

The samples produced only weeks apart showed to be the hardest to correctly discriminate, third inter-laboratory test. For ASTM E2927 comparison criterion, the samples produced two weeks apart were the only contribution to the false inclusion error rate.

For the equivalence test, when $\theta$ was calculated using the database, K1 vs Q1 and K2 vs Q3 were the pairs that were incorrectly associated. The false inclusion error rate was the highest when $\theta$ was calculated from the manufacturing plant. This happened to be the $\theta$ that produced the lowest amounts of false exclusions. 


\subsection{ASTM E2927 Error Rates for the M\&M Glass Database}

The newly created M\&M database was also used to assess the false exclusion error rate of the ASTM E2927 method. Since the comparison criterion in ASTM E2927 is used to compare $\mathrm{K}$ and $\mathrm{Q}$ fragments that were analyzed on the same day, the three fragments for each sample were used. Each fragment was treated as a Q sample and compared to the other two fragments, K, using the ASTM E2927 match criterion with a FRSD of 3\% applied to all elements. Table 23 shows the error rate for each fragment as well as problematic elements. Fragment 2 had the highest error rate at 2.6\%. This could be accounted to the fact that fragment 2 was often in the center of the ablation chamber. Differences were noted for how the laser interacted with fragments placed directly in the center of the chamber. For example the plasma produced by the laser was noticeably smaller for the fragments in the middle. Also the transient signal would drastically decrease. For some samples, the concentrations for the second fragment were slightly lower than the other two fragments. Once this was noticed, care was taken not to place fragments directly in the center of the ablation chamber.

Looking at the problematic elements, it can be observed that all fragments had an issue with ${ }^{208} \mathrm{~Pb}$. ASTM E2927 recommends using a FRSD of at least 3\%, but for some elements such as $\mathrm{Pb}$, the FRSD is often increased. Therefore it is recommended that labs validate their methods and adjust the FRSDs accordingly. 
Table 23 - False exclusion rate for M\&M Database

\begin{tabular}{|lccc|c|}
\hline & Fragment 1 & Fragment 2 & Fragment 3 & Average \\
\hline \# of Distinguished Pairs & 5 & 11 & 6 & 7 \\
Number of & 420 & 420 & 420 & \\
Comparisons & & & & \\
Discriminating & ${ }^{27} \mathrm{Al},{ }^{90} \mathrm{Zr}$, & & \\
Elements & ${ }^{137} \mathrm{Ba},{ }^{140} \mathrm{Ce},{ }^{208} \mathrm{~Pb}$ & ${ }^{139} \mathrm{La},{ }^{146} \mathrm{Nd},{ }^{180} \mathrm{Hf}$, & ${ }^{49} \mathrm{Ti},{ }^{88} \mathrm{Sr},{ }^{90} \mathrm{Zr},{ }^{208} \mathrm{~Pb}$ & \\
False Exclusion & & ${ }^{208} \mathrm{~Pb}$ & & 1.7 \\
Error Rate (\%) & 1.2 & 2.6 & & \\
\hline
\end{tabular}

\subsection{Conclusion}

This section focused on the evaluation of glass ICP-MS data using different match criteria. The application of the equivalence test to the EAWG inter-laboratory data resulted in a false exclusion error rate of $74-100 \%$ and false inclusion error rate of $0-12 \%$, depending on the how $\theta$ was calculated.

The results of this study support the use of the match criterion recommended in ASTM-E2927 and ASTM-E2330 ( $\pm 4 \mathrm{~s}$, FRSD at least $3 \%)$ When using this match criterion with the minimum FRSD to compare a large dataset of 420 samples, this match criterion produced an average false exclusion error rate of $1.7 \%$. 


\section{CHAPTER 10. Glass Interpretation Working Group Inter-Laboratory Studies}

This chapter details the results of the three Glass Interpretation Working Group (GIWG) inter-laboratory studies. The main focuses of this chapter are the evaluation of the performance of ASTM E2927 in terms of the false exclusion and false inclusion error rates, the comparison of how forensic labs interpret the same casework samples, and the use of a frequency calculation as a tool for interpretation. Though a variety of instrumentation was included, this study only focuses on the LA-ICP-MS. It should also be noted that likelihood ratios were tested as a tool for interpretation, but that is outside the scope of this research. For information about the likelihood ratio, please see Corzo et al [62].

\subsection{Samples}

The inter-laboratory test samples were all vehicle windshield glass. These samples originated from a salvage yard in Ruckersville, Virginia and were collected and analyzed by researchers at Florida International University prior to being used in the inter-laboratory tests. The inter-laboratory tests were designed as mock cases in which participants were asked to analyze and compare Q glass samples to $\mathrm{K}$ glass samples. The samples chosen for each inter-laboratory study are presented in Table 24. Since windshields are composed of two glass panes held together by a polymer film, each $\mathrm{K}$ glass was sent out as two samples (K1 inner, K1 outer). 
Table 24 - GIWG inter-laboratory test samples

\begin{tabular}{|c|c|c|c|c|c|}
\hline \multicolumn{6}{|c|}{ Inter-laboratory Test 1} \\
\hline Sample & Make & Model & Year & VIN & Remarks \\
\hline $\mathrm{K} 1_{\text {(inner \& outer) }}$ & Mitsubishi & Galant & 2009 & 4АЗАВ36F39Е024088 & \\
\hline $\mathrm{K} 2$ (inner \& outer) & Subaru & Impreza & 2008 & JF1GE61658H503418 & \\
\hline Q1 & Mitsubishi & Galant & 2009 & 4АЗАВ36F39E029145 & Similar to $\mathrm{K} 1$ \\
\hline Q2 & Subaru & Impreza & 2008 & JF1GE61658H503418 & K2 outer \\
\hline \multicolumn{6}{|c|}{ Inter-laboratory Test 2} \\
\hline Sample & Make & Model & Year & VIN & Remarks \\
\hline $\mathrm{K} 1_{\text {(inner \& outer) }}$ & Honda & Civic & 2006 & 2HGFG21506H707035 & \\
\hline Q1 & Honda & Civic & 2006 & 2HGFG21506H707035 & $\begin{array}{l}2 \text { fragments } \mathrm{K} 1 \text { inner } \\
1 \text { fragment } \mathrm{K} 1 \text { outer }\end{array}$ \\
\hline Q2 & BMW & 2 Series & 2014 & WBA1F5C58EVV98871 & \\
\hline \multicolumn{6}{|c|}{ Inter-laboratory Test 3} \\
\hline Sample & Make & Model & Year & VIN & Remarks \\
\hline $\mathrm{K} 1_{\text {(inner \& outer) }}$ & Honda & Civic & 2007 & 2HGFG12607H511521 & \\
\hline Q1 & Honda & Civic & 2007 & 2HGFG12607H511521 & K1 outer \\
\hline Q2 & Mercedes & R-Class & 2009 & 4JGCB65E59A094913 & \\
\hline
\end{tabular}

\subsection{Inter-laboratory Test 1}

This test was organized to evaluate and compare the current analytical schemes used by the participating laboratories, to examine the tools that are presently being used 
in interpreting results, and to determine if elemental glass databases could be combined. The use of ASTM E2927 was encouraged, but not enforced.

Each laboratory received six samples labeled as K1 inner, K1 outer, K2 inner, K2 outer, Q1, and Q2. Each sample consisted of three full thickness fragments approximately $3-5 \mathrm{~mm}$ in size. Laboratories were instructed to follow the analytical scheme that they use for casework and to analyze NIST1831 as a quality control standard. They were asked to submit a description of the comparison criteria used and a report with their interpretation of the data. The samples were selected to test how well the analytical scheme used by each lab can discriminate samples from very different vehicles (K1 and Q2, K2 and Q1), discriminate samples from similar vehicles (K1 and Q1), and associate samples that originate from the same windshield pane (K2 and Q2).

\subsubsection{Analytical Schemes}

The analytical scheme differed between laboratories. Some laboratories measured thickness and RI before performing elemental analysis while others only used elemental analysis. Moreover, the comparison criteria, element menu, and calibration technique used differed slightly between laboratories. Though the calibration technique differed, all labs used ${ }^{29} \mathrm{Si}$ as an internal standard. Each lab previously validated their instrumentation and method.

Laboratory A measured both thickness and RI. They used the ASTM E2927 comparison criterion but with a minimum FRSD that was defined per element. The FRSD was based off a previous study that evaluated the elemental variation within a single float glass pane and between different float glass sources. Elements with a higher variability 
have a higher minimum FRSD. The FRSD ranged from $4 \%$ all the way to $12.5 \%$. Laboratory A also included more elements than any other lab: ${ }^{7} \mathrm{Li},{ }^{25} \mathrm{Mg},{ }^{27} \mathrm{Al},{ }^{42} \mathrm{Ca},{ }^{49} \mathrm{Ti}$, ${ }^{55} \mathrm{Mn},{ }^{59} \mathrm{Co},{ }^{85} \mathrm{Rb},{ }^{88} \mathrm{Sr},{ }^{89} \mathrm{Y},{ }^{90} \mathrm{Zr},{ }^{95} \mathrm{Mo},{ }^{118} \mathrm{Sn},{ }^{121} \mathrm{Sb},{ }^{137} \mathrm{Ba},{ }^{139} \mathrm{La},{ }^{140} \mathrm{Ce},{ }^{146} \mathrm{Nd},{ }^{182} \mathrm{~W}$, ${ }^{208} \mathrm{~Pb},{ }^{232} \mathrm{Th},{ }^{238} \mathrm{U}$. Though ${ }^{39} \mathrm{~K}$ and ${ }^{57} \mathrm{Fe}$ were monitored, these elements are excluded from casework since they caused inconsistent results during method development. This lab used the software PlasmaLab, which utilizes a blank and glass standards, NIST614 and NIST612, to determine the concentration in the samples.

Laboratory B only performed elemental analysis. They used the ASTM E2927 comparison criterion with a minimum FRSD of $3 \%$ applied to all elements. This lab analyzed 17 elements for comparative purposes: ${ }^{7} \mathrm{Li},{ }^{25} \mathrm{Mg},{ }^{27} \mathrm{Al},{ }^{39} \mathrm{~K},{ }^{42} \mathrm{Ca},{ }^{57} \mathrm{Fe},{ }^{49} \mathrm{Ti}$, ${ }^{55} \mathrm{Mn},{ }^{85} \mathrm{Rb},{ }^{88} \mathrm{Sr},{ }^{90} \mathrm{Zr},{ }^{137} \mathrm{Ba},{ }^{139} \mathrm{La},{ }^{140} \mathrm{Ce},{ }^{146} \mathrm{Nd},{ }^{180} \mathrm{Hf},{ }^{208} \mathrm{~Pb} .{ }^{118} \mathrm{Sn}$ was monitored only to determine float side. This lab used GLITTER's single-point calibration with FGS2 as the calibrator.

Laboratory $\mathrm{C}$ measured both thickness and RI. If differences were found, they did not perform elemental analysis. Like laboratory B, they also used the ASTM E2927 comparison criterion with a minimum FRSD of $3 \%$ applied to all elements. This lab analyzed 18 elements: ${ }^{7} \mathrm{Li},{ }^{25} \mathrm{Mg},{ }^{27} \mathrm{Al},{ }^{39} \mathrm{~K},{ }^{42} \mathrm{Ca},{ }^{57} \mathrm{Fe},{ }^{49} \mathrm{Ti},{ }^{55} \mathrm{Mn},{ }^{85} \mathrm{Rb},{ }^{88} \mathrm{Sr},{ }^{90} \mathrm{Zr},{ }^{118} \mathrm{Sn}$, ${ }^{137} \mathrm{Ba},{ }^{139} \mathrm{La},{ }^{140} \mathrm{Ce},{ }^{146} \mathrm{Nd},{ }^{180} \mathrm{Hf},{ }^{208} \mathrm{~Pb}$. When it came to comparing the $\mathrm{Q}$ mean to the comparison interval, this laboratory was the only one to round the numbers. There were two instances where a comparison pair was distinguished by only one element, ${ }^{88} \mathrm{Sr}$ or ${ }^{7} \mathrm{Li}$. In both cases, the $\mathrm{Q}$ means fell just outside the comparison interval with the difference in concentration being in the first decimal place. This situation is referred to as the "fall off the cliff" effect. By rounding the numbers to the nearest whole number, this 
lab found the comparisons to be indistinguishable. When discussing the "fall off the cliff" effect with the participating analysts, most responded that they would also label the samples as indistinguishable or re-analyze the samples if time allowed. This lab used GLITTER's single-point calibration with NIST612 as the calibrator.

Laboratory D measured thickness and RI. This lab used a different comparison criterion. They used the $\mathrm{K}$ sample to create a comparison interval for each element where the upper limit was $\mathrm{K}$ mean $\times(1+4 \times$ FRSD $)$ and the lower limit was $\mathrm{K}$ mean $\div(1+4 \times$ FRSD). They did not use the SD of the K sample, and instead used only a FRSD that was determined in their laboratory based on the inter-day variability of a control glass. The minimum FRSD was 3\%, but increased depending on the inter-day variability of each element. This lab used 18 elements in their comparisons: ${ }^{7} \mathrm{Li},{ }^{23} \mathrm{Na},{ }^{25} \mathrm{Mg},{ }^{27} \mathrm{Al},{ }^{39} \mathrm{~K},{ }^{42} \mathrm{Ca}$, ${ }^{57} \mathrm{Fe},{ }^{49} \mathrm{Ti},{ }^{55} \mathrm{Mn},{ }^{85} \mathrm{Rb},{ }^{88} \mathrm{Sr},{ }^{90} \mathrm{Zr},{ }^{137} \mathrm{Ba},{ }^{139} \mathrm{La},{ }^{140} \mathrm{Ce},{ }^{146} \mathrm{Nd},{ }^{180} \mathrm{Hf},{ }^{208} \mathrm{~Pb} .{ }^{118} \mathrm{Sn}$ was monitored only to determine float side. This lab evaluated the data two ways. They used GLITTER's single-point calibration with FGS2 as the calibrator, and then re-evaluated the data with NIST612 as the calibrator.

\subsubsection{Results and Interpretation}

Focusing only on the elemental data submitted by each lab, the following results were found. Four labs submitted elemental data for the comparisons of K1 inner with Q1 and Q2; one lab found differences between these samples with thickness measurements and RI and therefore did not analyze K1 inner with LA-ICP-MS. All labs that submitted data correctly distinguished $\mathrm{K} 1$ inner from Q1 with all labs detecting a difference in ${ }^{57} \mathrm{Fe}$ and ${ }^{137} \mathrm{Ba}$. Some also detected differences in ${ }^{49} \mathrm{Ti},{ }^{88} \mathrm{Sr}$, and ${ }^{90} \mathrm{Zr}$. All labs also correctly 
distinguished K1 outer from Q2, K2 outer from Q1, and K2 inner from Q1. However, for the comparison of $\mathrm{K} 1$ outer with Q1, most labs could not detect reproducible elemental differences. Since K1 and Q1 originated from very similar vehicles (same make, model, year with VINs less than 5000 apart), it is possible that the glass from these windshield panes were produced at the same plant around the same time.

All labs correctly associated K2 outer with Q2. All labs also associated K2 inner with Q2 even though Q2 came from the outer pane of this windshield. From a forensic point of view, the participants correctly associated Q2 with the windshield of the vehicle it originated from.

The elemental concentrations for NIST1831 were compared between labs and to the reported value to determine whether glass databases created by different laboratories could be combined into one large database. The following element menu was used: ${ }^{7} \mathrm{Li}$, ${ }^{25} \mathrm{Mg},{ }^{27} \mathrm{Al},{ }^{39} \mathrm{~K},{ }^{42} \mathrm{Ca},{ }^{57} \mathrm{Fe},{ }^{49} \mathrm{Ti},{ }^{55} \mathrm{Mn},{ }^{85} \mathrm{Rb},{ }^{88} \mathrm{Sr},{ }^{90} \mathrm{Zr},{ }^{137} \mathrm{Ba},{ }^{139} \mathrm{La},{ }^{140} \mathrm{Ce},{ }^{146} \mathrm{Nd},{ }^{208} \mathrm{~Pb}$. Differences were seen between the different calibration techniques for the concentrations of ${ }^{25} \mathrm{Mg},{ }^{39} \mathrm{~K}$, ${ }^{49} \mathrm{Ti}$, and ${ }^{57} \mathrm{Fe}$, Figure 46 . Though calibrated the same way, Lab $\mathrm{C}$ and the NIST612 calibrated data from Lab D differed for ${ }^{25} \mathrm{Mg},{ }^{39} \mathrm{~K}$, and ${ }^{49} \mathrm{Ti}$. It should also be noted that Lab C reported issues with their LA-ICP-MS system that led to unusual high RSDs for all samples analyzed. The differences in ${ }^{39} \mathrm{~K}$ have been seen in our research group before. The issue is thought to be caused by the distribution of this element in the NIST612 glass. As can be seen in Figure $45,{ }^{39} \mathrm{~K}$ in NIST612 (dark blue) is more concentrated at the surface. Overtime, as the laser creates a crater in the glass, the ${ }^{39} \mathrm{~K}$ signal drastically decreases. The other elements in NIST612 do not exhibit this behavior as can be seen by the ${ }^{55} \mathrm{Mn}$ signal (light blue). The ${ }^{39} \mathrm{~K}$ in FGS2 (black) does not display 
this behavior either, which leads to the conclusion that the problem is with the NIST612 glass sample and not the instrument.

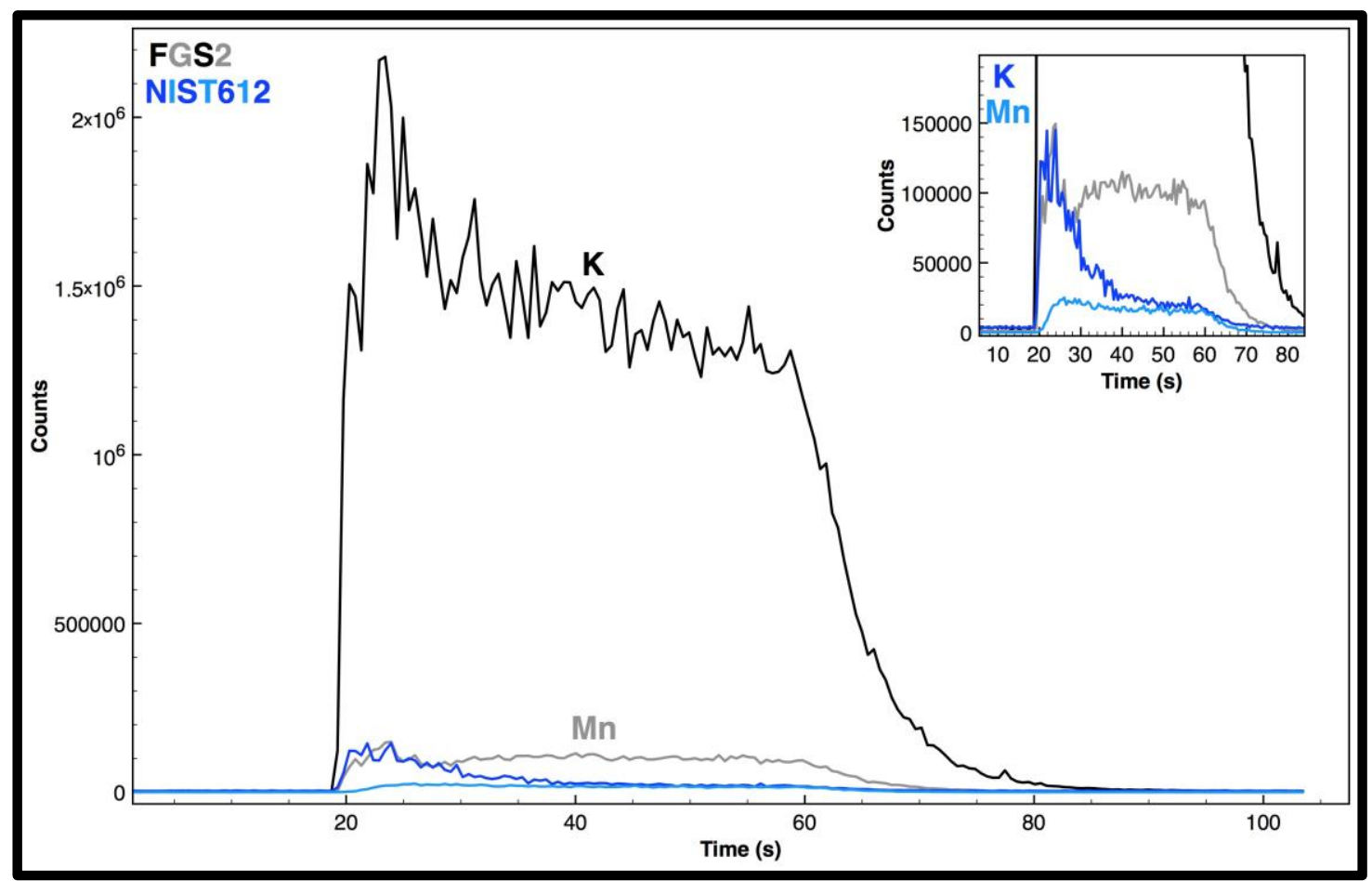

Figure 45 - Transient signal of K in NIST612 and FGS2

Similarities between Labs B, D, and E were seen for all elements when these labs used single point calibration with FGS2 as the calibrator. Interestingly all labs detected a concentration for ${ }^{55} \mathrm{Mn},{ }^{90} \mathrm{Zr},{ }^{140} \mathrm{Ce}$, and ${ }^{208} \mathrm{~Pb}$ that was below the reported value, Figure 46. This could indicate a systematic negative bias.

It was concluded that databases from labs using FGS2 to calibrate could potentially be combined without limiting the element menu. Since both the FIU M\&M database and database 5, Table 19, are calibrated this way, these databases were combined and the combined database was used for the rest of the inter-laboratory studies.

It may be possible to combine databases that were calibrated with NIST612 and by external calibration with FGS2 calibration database, but the element menu would have 
to be restricted to only the elements that produced similar concentrations regardless of the calibration technique used. To test this, ${ }^{25} \mathrm{Mg},{ }^{39} \mathrm{~K},{ }^{49} \mathrm{Ti}$, and ${ }^{57} \mathrm{Fe}$ were removed from the database. Each lab's data for K1 and K2 were compared to the duplicate glass in the database: Sample 110 inner and outer and Sample 201 inner (the outer pane of this sample was removed from the database because it was found to be indistinguishable from the inner pane). All of the K1 inner data was found to be indistinguishable with Sample 110 inner. The same was true for the comparison of K2 inner and outer with Sample 201. The only difference was seen in the K1 outer data from Lab D with Sample 110 Outer. This pair was distinguished by Rb. This was just an exercise to demonstrate the potential of using a central database and combining databases by limiting the element menu. For the remaining studies, the full element menu was used. 


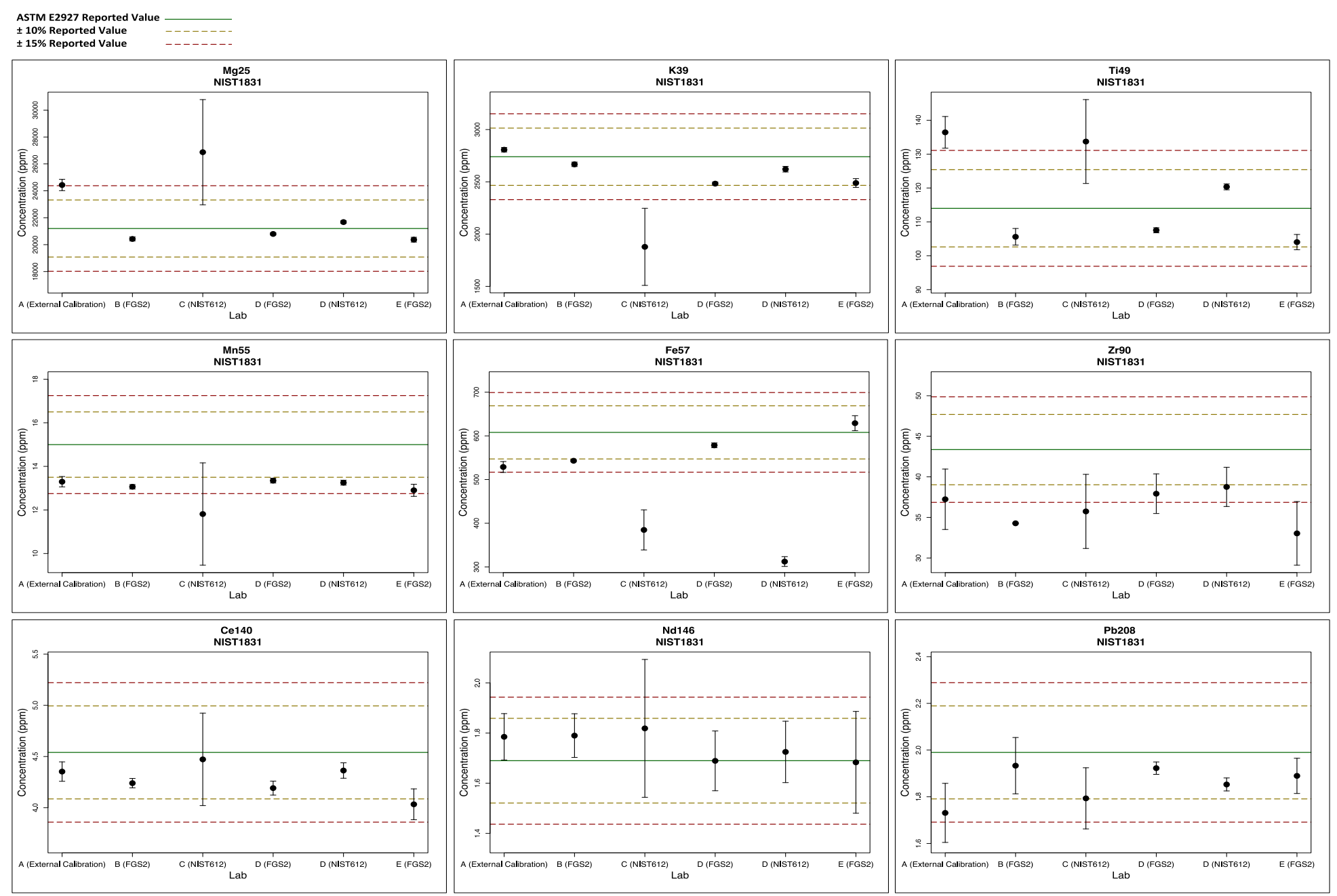

Figure 46 - Inter-lab comparison of NIST1831. Shown are each labs calculated mean \pm the standard deviation of the measurement for select elements 


\subsubsection{Interpretation for First Inter-Laboratory Test}

Laboratory B did not provide an interpretation for the comparison of $\mathrm{K} 2$ inner and Outer with Q2 comparison pairs, Laboratories A and C did not include any statements about the strength of the association. These labs stated that Q2 was indistinguishable in the measured physical and chemical properties from $\mathrm{K} 2$ inner and $\mathrm{K} 2$ outer. Therefore, Q2 could have originated from the same source as K2 or from another source of glass produced by the same manufacturer exhibiting the same physical and chemical properties. Laboratories $\mathrm{D}$ and $\mathrm{E}$ used statistics based on their databases to aid in their interpretation. Laboratory D used an estimated random match probability stating that their database is not large enough to give a value for one given sample. This probability of a random match was calculated based on the false inclusion rate of their comparison criterion and reported to be $0.1 \%$. They also used a verbal scale that is based on how common or uncommon the measured properties are in the population. Since K2 and Q2 were indistinguishable in thickness, RI, and elemental analysis by LA-ICP-MS, they concluded that the results showed very strong support for the hypothesis that Q2 originated from one of the two panes of K2. Laboratory E calculated a likelihood ratio using a database populated with Known samples from casework and used a verbal scale to report their results. The lab noted that their likelihood ratios should be interpreted as a score, since the values are un-calibrated. Based on their $L R$ score, Lab E concluded that the results of the investigation are appreciably more probable when the Q2 fragments originate from the windowpane to which the reference glass, $\mathrm{K} 2$, has belonged. 


\subsubsection{Frequency of Occurrence for the First Inter-Laboratory Exercise}

The random match probability of the Database 5, the FIU database, and the combined database was calculated to be $0.06 \%$ (44/68265), $0.24 \%$ (132/55278), and $0.08 \%(186 / 246753)$ respectively. The random match probability is higher for the FIU database, because this database includes many vehicles that have the same make, model, and/or year. However, after combining the databases, the random match probability is closer to $0.13 \%$, which has been reported for other casework databases.

The use of a common database to determine the frequency of a specific elemental composition was tested by comparing each lab's measurements for K1 outer and K2 (the $\mathrm{K}$ samples of indistinguishable pairs) to the combine database using the comparison criterion described in section 7.7 .

A description of the samples in the FIU vehicle database can be found in the appendix. Database 5 is made up of the Ks and Qs from casework, so the true identity of the samples is unknown. K1 outer originated from sample 110. When the outer pane of sample 110 was compared to the other 703 samples in the combined database, it was found to be indistinguishable with the outer pane of samples 65 and 66. Therefore if it expected that when comparing the data for $\mathrm{K} 1$ outer from the labs to the database, they will also be indistinguishable with these samples as well as sample 110. Interestingly, all of the samples found to be indistinguishable came from 2009 Mitsubishi Galants.

Labs B, D (FGS2 calibrated), and E had similar frequencies. The concentrations for K1 outer from these three laboratories were found to be indistinguishable with the outer pane of sample 110, the outer pane of sample 66, and the outer pane of sample 65 . Lab B's K1 outer was also found to be indistinguishable with the inner pane of sample 
111. Sample 111 was distinguished from sample 110 and Labs D and E by ${ }^{39} \mathrm{~K}$. All elemental comparisons for $\mathrm{K} 1$ outer, $\mathrm{K} 1$ inner and $\mathrm{K} 2$ to their duplicate in the database can be found in the Appendix. Figure 47 shows the comparison of the lab's K1 data to sample 110 using the comparison criteria for a few elements. Also shown in the figure are the outer panes of samples 65,66 , and the inner pane of sample 111 . When looking at ${ }^{39} \mathrm{~K}$, it can be seen that sample 111 has a higher concentration that just falls outside the comparison interval created using sample 110. Lab B determined a concentration of ${ }^{39} \mathrm{~K}$ in $\mathrm{K} 1$ outer that is higher than the database sample 110. Thus causing K1 outer to be indistinguishable with sample 111. The concentration of ${ }^{39} \mathrm{~K}$ for Labs D and $\mathrm{E}$ is right in line with the concentration of sample 110. Thus these samples would be distinguished from sample 111. This is once again an example of the fall off the cliff effect. 
Li7

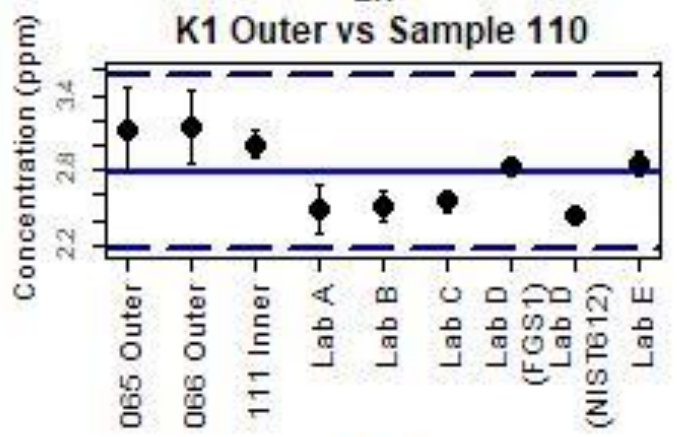

K39

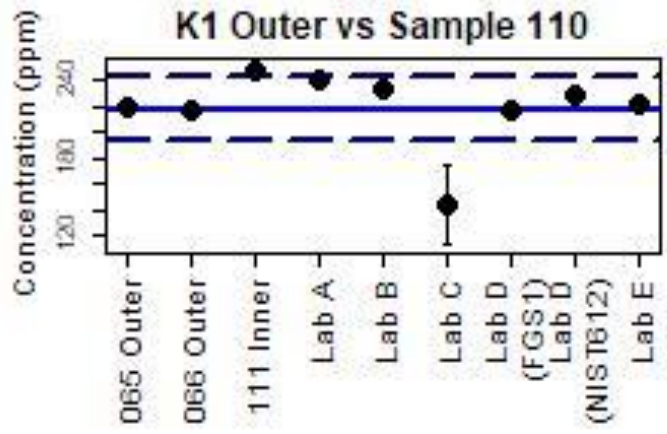

Mg25

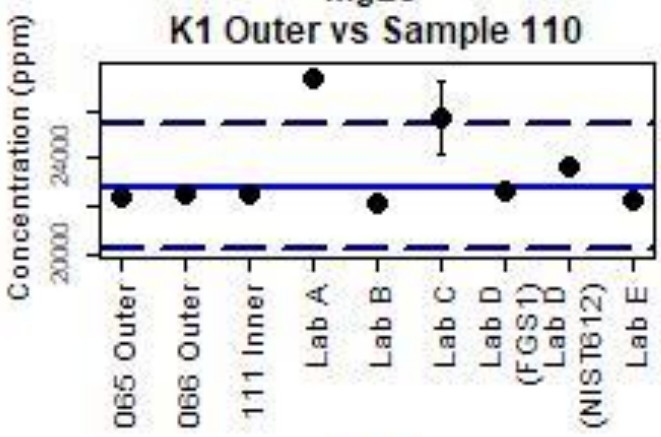

Rb85

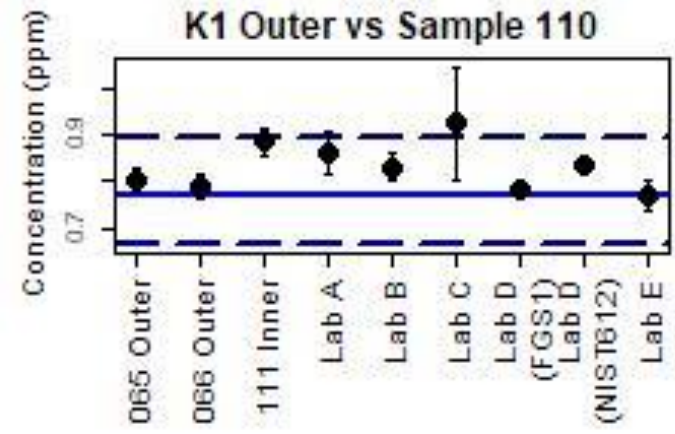

Figure 47 - Comparison of each lab's K1 outer to its duplicate in the database

The data for K1 outer from Labs A, C, and the NIST612 calibrated data from Lab

D were found to be distinguished from every sample in the database. This is caused by differences in how these labs calibrated their data in comparison to how the database was calibrated. Differences were seen for the concentrations of ${ }^{25} \mathrm{Mg},{ }^{39} \mathrm{~K},{ }^{49} \mathrm{Ti},{ }^{57} \mathrm{Fe}$, and ${ }^{85} \mathrm{Rb}$. The frequency of K1 outer is $3 / 703$ for Labs B and D (FGS2 calibrated), 4/703 for Lab E, and 0/703 for Labs A, C, and D (NIST612 calibrated).

K2 originated from sample 201. When comparing sample 201 to the database, it was found to be distinguished from all the samples. Labs B, D (FGS2 calibrated), and E found their K2 measurements to be indistinguishable with only sample 201 in the database. The data from Labs A, C, and the NIST612 calibrated data from Lab D were found to be distinguished from every sample in the database. The frequency of K2 is 1/703 for Labs 
B, D (FGS2 calibrated), and E. For Labs A, C, and D (NIST612 calibrated), the frequency is $0 / 703$.

\subsection{Inter-laboratory Test 2}

This test served several functions: to evaluate the performance of ASTM E2927$17 \mathrm{e} 1$, to examine the use of a common database for interpreting results when all labs use the same calibration technique, and to assess how laboratories report results when a $\mathrm{Q}$ sample contains two different glasses that are indistinguishable with a single $\mathrm{K}$ source. The laboratories were instructed to use single point calibration with FGS2 as the calibrator and the comparison criterion in ASTM E2927-17e1. The following element menu was to be used: ${ }^{7} \mathrm{Li},{ }^{25} \mathrm{Mg},{ }^{27} \mathrm{Al},{ }^{39} \mathrm{~K},{ }^{42} \mathrm{Ca},{ }^{57} \mathrm{Fe},{ }^{49} \mathrm{Ti},{ }^{55} \mathrm{Mn},{ }^{85} \mathrm{Rb},{ }^{88} \mathrm{Sr},{ }^{90} \mathrm{Zr},{ }^{137} \mathrm{Ba}$,

${ }^{139} \mathrm{La},{ }^{140} \mathrm{Ce},{ }^{146} \mathrm{Nd},{ }^{208} \mathrm{~Pb}$ or combined average of ${ }^{206} \mathrm{~Pb},{ }^{207} \mathrm{~Pb},{ }^{208} \mathrm{~Pb}$. Though ASTM E2927 also suggests using ${ }^{180} \mathrm{Hf}$, this element was excluded because it is highly correlated with ${ }^{90} \mathrm{Zr}$. The participants were instructed to perform elemental analysis on all the samples even if they found them to be distinguished using RI or thickness.

The number of participants increased to nine labs for this exercise. Each laboratory received four samples labeled K1 inner, K1 outer, Q1, and Q2. Each K sample consisted of three full thickness fragments approximately 3-5 $\mathrm{mm}$ in size. To better mimic actual casework, the $\mathrm{Q}$ samples consisted of three thin, irregularly shaped fragments of approximately $1 \mathrm{~mm}$ in size. Though they were of similar thickness and color, the inner and outer panes of K1 differed greatly in elemental concentration. Q1 consisted of two fragments from the inner pane and one fragment from the outer. Q2 was from another vehicle that should easily be discriminated from both panes of $\mathrm{K} 1$. The 
laboratories were also sent FGS2 and FGS1 to serve as a calibrator and quality control, respectively. To account for instrumental drift, participants were asked to measure five replicates on FGS2 at the beginning and end of their analysis. Participants were also told to measure at least five replicates on FGS1 and a minimum of three replicates on each glass fragment.

For comparing the $\mathrm{K}$ and $\mathrm{Q}$ samples, the replicate measurements on each fragment of a $\mathrm{K}$ glass pane (K1 inner or $\mathrm{K} 1$ outer) were grouped together. Each $\mathrm{Q}$ fragment was to be considered separately (Q1.1, Q1.2, Q1.3). Participants were asked to submit a report with their interpretation of the data.

The participants of the first inter-laboratory study mentioned that including a case scenario would aid in writing a report. Thus, as requested, the participants of the second study were given a case scenario that stated a vehicle, 2006 Honda Civic, was found with a broken front windshield, K1. There were two possible suspects. Three glass fragments, Q1, were found on the clothing of suspect \#1. Three glass fragments were found on the clothing of suspect \#2, Q2.

\subsubsection{Evaluation of ASTM E2927-17e1 as a Method for Glass Comparisons}

Since participants were asked to deviate from their validated methods, ASTM E2927 with a FRSD of 3\% for all elements was used to compare all Ks and Qs. For Q1, two of the fragments originated from $\mathrm{K} 1$ inner and the third from K1 outer. Q2 was sampled from the outer pane of another vehicle that was very different from both panes of K1. There were a total of 27 comparisons that should have resulted in an association. 


\section{(2 Q1 Fragments with K1 inner +1 Q1 Fragment with K1 outer) $\times 9$ Participating \\ Laboratories $=27$ Comparisons}

There were no false exclusions: all labs correctly associated two Q1 fragments with K1 inner and one Q1 fragment with K1 outer.

There were 81 comparisons that should have resulted in an exclusion.

(1 Q1 Fragment with K1 inner +2 Q1 Fragments with K1 outer +3 Q2 Fragments with

K1 inner +3 Q2 Fragments with K1 outer) $\times 9$ Participating Laboratories $=81$

\section{Comparisons}

There were no false inclusions: all labs correctly distinguished Q2 from both K1 panes, one Q1 fragment from K1 inner, and two Q1 fragments from K1 outer.

\subsubsection{Interpretation for Second Inter-Laboratory Exercise}

The interpretation of the pairs found to be indistinguishable improved compared to the first inter-laboratory study, with more labs using significance statements based on statistical analysis with a database and/or a verbal scale. However, there were some disagreements in the strength of the association. There were still two laboratories reporting only that the indistinguishable pairs could have originated from the same source with only one of these labs mentioning that this sample could be from another source produced at the same manufacturing plant around the same time. This lab also assessed the rarity of the glass by comparing $\mathrm{K} 1$ outer's elemental profile, refractive index, and thickness to their database. Though there were some glasses with a similar elemental profile, all of these were distinguished from K1 outer by either RI or thickness. One lab stated that they do not have an official verbal scale, but have some wordings that they 
use. For this comparison, the results strongly suggest that the glass fragments found on the clothing of suspect \# 1 (Q1) are originally from the front windshield of the Honda Civic (K1). Strongly suggests is used when they find case samples that are indistinguishable in refractive index and LA-ICP-MS analysis. Three labs used the fact that Q1 contained glasses that were indistinguishable with both $\mathrm{K} 1$ panes to increase the significance of the elemental analysis. Two of these labs reported that there is very strong indication that the Q1 fragments originated from the same source as K1. The other lab reported that the results are far more probable when these fragments (Q1) originate from the window to which the reference glass $\mathrm{K} 1$ has belonged, than when they originate from another random float glass or glass object. One lab used a verbal scale that did not account for multiple Q fragments matching with the K source. This lab reported that the Q1 fragments very likely originated from the same source as K1. They also stated that other sources of glass with similar characteristics are limited. This interpretation was based on these glass samples sharing the same class characteristics with no significant differences in terms of trace elemental composition and refractive index. Two laboratories did not provide an interpretation of the results.

\subsubsection{Frequency for Second Inter-Laboratory Exercise}

The use of the same calibration technique improved the frequency calculations. K1 originated from sample 78 . When the inner pane of sample 78 was compared to the other 703 samples in the combined database, it was distinguished from all samples. The data for $\mathrm{K} 1$ inner for all but two labs, Labs $\mathrm{C}$ and $\mathrm{F}$, was indistinguishable with only the inner pane of sample 78. The data for K1 inner from Lab C and Lab F was distinguished 
from this sample by either ${ }^{139} \mathrm{La}$ or ${ }^{85} \mathrm{Rb}$. When comparing Lab C's concentration for ${ }^{139} \mathrm{La}$ and Lab F's concentration for ${ }^{85} \mathrm{Rb}$ to the concentration determined by the other laboratories, both are extreme values (lowest/highest), which were different by more than $15 \%$ of the inter-laboratory average. The frequency for $\mathrm{K} 1$ inner for is $1 / 703$ for Labs A, B, D, E, G, H, and I and 0/703 for Labs C and F.

When the outer pane of sample 78 was compared to the database, it was indistinguishable with the outer pane of samples 21 and 45. Most elements had very similar concentrations between the samples.

The data for $\mathrm{K} 1$ outer for all labs except Lab C and Lab F was found to be indistinguishable with the outer pane of sample 78 in the database. For Lab C and Lab F the same issue seen for K1 inner was seen. Labs A, B, D, E, G, H, and I also found K1 outer to be indistinguishable with the outer pane of sample 21. Only labs B and G found their data for $\mathrm{K} 1$ outer to be indistinguishable with sample 45. This sample was distinguished from $\mathrm{K} 1$ outer by ${ }^{208} \mathrm{~Pb}$ for most labs with some also detecting differences in ${ }^{88} \mathrm{Sr},{ }^{139} \mathrm{La}$ for Lab C, and ${ }^{85} \mathrm{Rb}$ for Lab F. Looking at the comparison of each lab's K1 outer to the outer pane of sample 78, it can be seen that all lab's have an average for $\mathrm{Pb}$ that is less than the database average. The FIU database has a high inter-day variation for $\mathrm{Pb}$, which may not be properly accounted for in the current match criteria. This may also be an indication of an element that should be eliminated from the element menu. The frequency for $\mathrm{K} 1$ outer is $0 / 703$ for Labs $\mathrm{C}$ and $\mathrm{F}, 1 / 703$ for Lab E, and 2/703 for Labs A, $\mathrm{D}, \mathrm{H}$, and I, and 3/703 for Labs B and $\mathrm{G}$. 


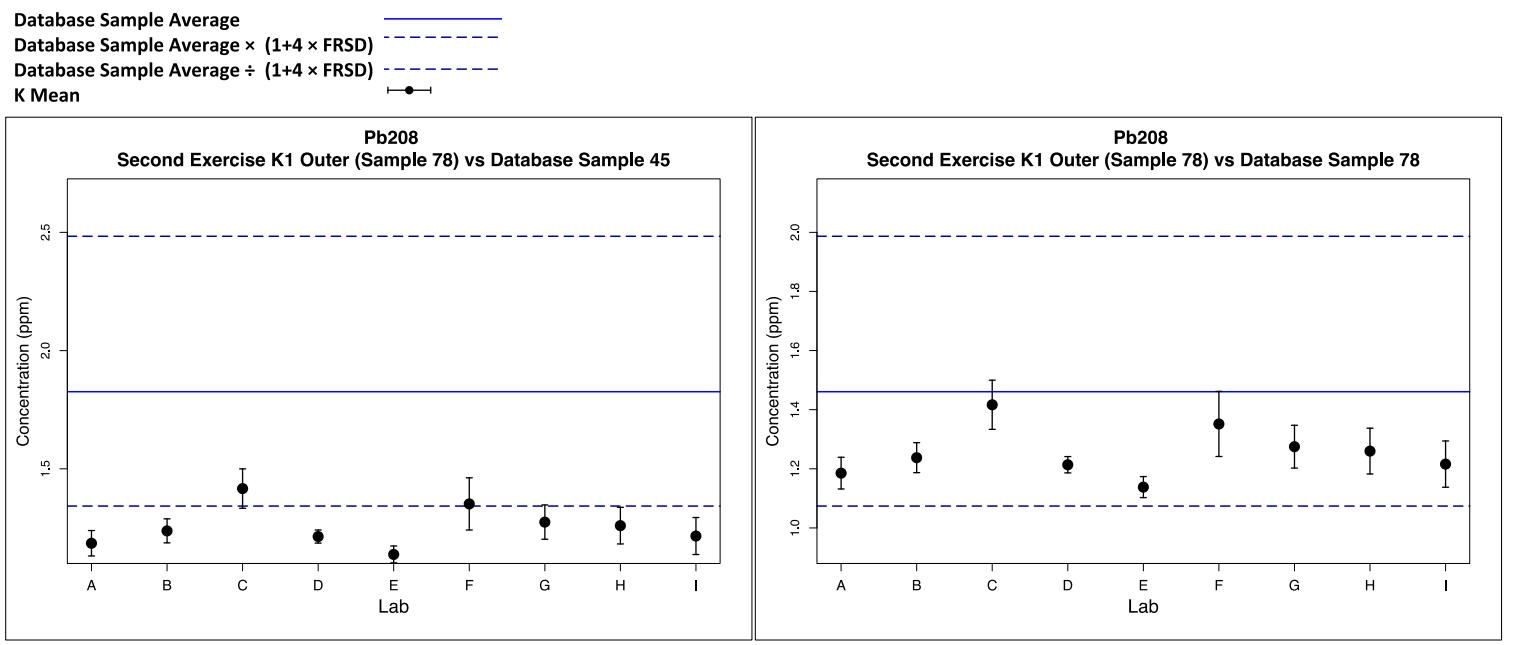

Figure 48 - Comparison of each lab's K1 outer to sample 45 and sample 78 in the database

\subsection{Third Inter-Laboratory Exercise}

This test was designed to assess the use of a common database for calculating the frequency. In the second inter-laboratory exercise, participants were sent sample 78 as K1. When comparing the lab's K1 outer to the combined database, it was found to be indistinguishable by most labs with the outer pane of sample 21 . For the third interlaboratory exercise, participants were sent sample 21 as $\mathrm{K} 1$ to test if it has the same or similar frequency as sample 78. The laboratories were instructed to use single point calibration with FGS2 as the calibrator and use the comparison criterion recommended in ASTM E2927-17e1.

Ten laboratories participated in this exercise. Each laboratory received four samples labeled K1 inner, K1 outer, Q1, and Q2. The Ks and Qs were similar in size to those used in the second inter-laboratory study. Q1 originated from the same pane as K1 outer. Q2 was sampled from the outer pane of another vehicle. The glass manufacturer information was available for both vehicles. The glass information included the name of the vehicle manufacturer, Honda or Mercedes, which is an indication that these are most 
likely OEM windshields. Both windshields were manufactured by PPG, were SOLARGREEN M413, and had the same DOT code, DOT 18. Despite originating from vehicles of different makes and produced two years apart, both windshields had similar elemental profiles in their outer panes. However, when analyzed by LA-ICP-MS, reproducible differences could be detected in ${ }^{57} \mathrm{Fe}$. Like in the second inter-laboratory study, K1 inner and K1 outer differed in their elemental profiles.

The participants of the third study were given a case scenario that stated a vehicle, 2007 Honda Civic, was found with a broken front windshield, K1. There were two possible suspects. Three glass fragments, Q1, were found on the clothing of suspect \#1. Three glass fragments were found on the clothing of suspect \#2, Q2.

The participants were told to follow the same instructions as the second inter-laboratory exercise. For reporting the results, a verbal scale was sent to the participants. No information was given about how to use the scale. Participants were asked what category they would use to report their findings considering only the LA-ICP-MS data:

Level 1: Physical/Fracture Match

Level 2: Association with highly discriminating characteristics

Level 3: Association with discriminating characteristics

Level 4: Association with limitations

Level 5: Inconclusive

Level 6: Elimination/Exclusion 


\subsubsection{Evaluation of ASTM E2927}

The comparison criterion in ASTM E2927-16e1 with a FRSD of 3\% for all elements was used to compare all Ks and Qs. There were 30 comparisons that should have resulted in an association.

(3 Q1 Fragments with K1 outer $) \times 10$ Participating Laboratories $=30$ Comparisons

There were no false exclusions. All labs correctly associated all Q1 fragments with $\mathrm{K} 1$ outer.

There were 90 comparisons that should have resulted in an exclusion.

(3 Q1 Fragments with K1 inner +3 Q2 Fragments with K1 inner +3 Q2 Fragments with K1 outer $) \times 10$ Participating Laboratories $=90$ Comparisons

Figure 49, provides an example of the ASTM E2927-16e1 match criterion for the comparison of K1 outer and Q2.1. Even though Q2 had a similar elemental composition to $\mathrm{K} 1$ outer, all of the participating laboratories were able to detect differences in ${ }^{57} \mathrm{Fe}$ with some also detecting differences in ${ }^{90} \mathrm{Zr}$ and ${ }^{137} \mathrm{Ba}$. Figure 49 also depicts the interlaboratory average for each element with limits set at $10 \%$ and $15 \%$ of the interlaboratory average. Lab C's measurements for ${ }^{139} \mathrm{La}$ improved since the second interlaboratory exercise and are now closer to the concentration determined by the other labs. The same is true for Lab's F measurements for ${ }^{85} \mathrm{Rb}$, not shown. 


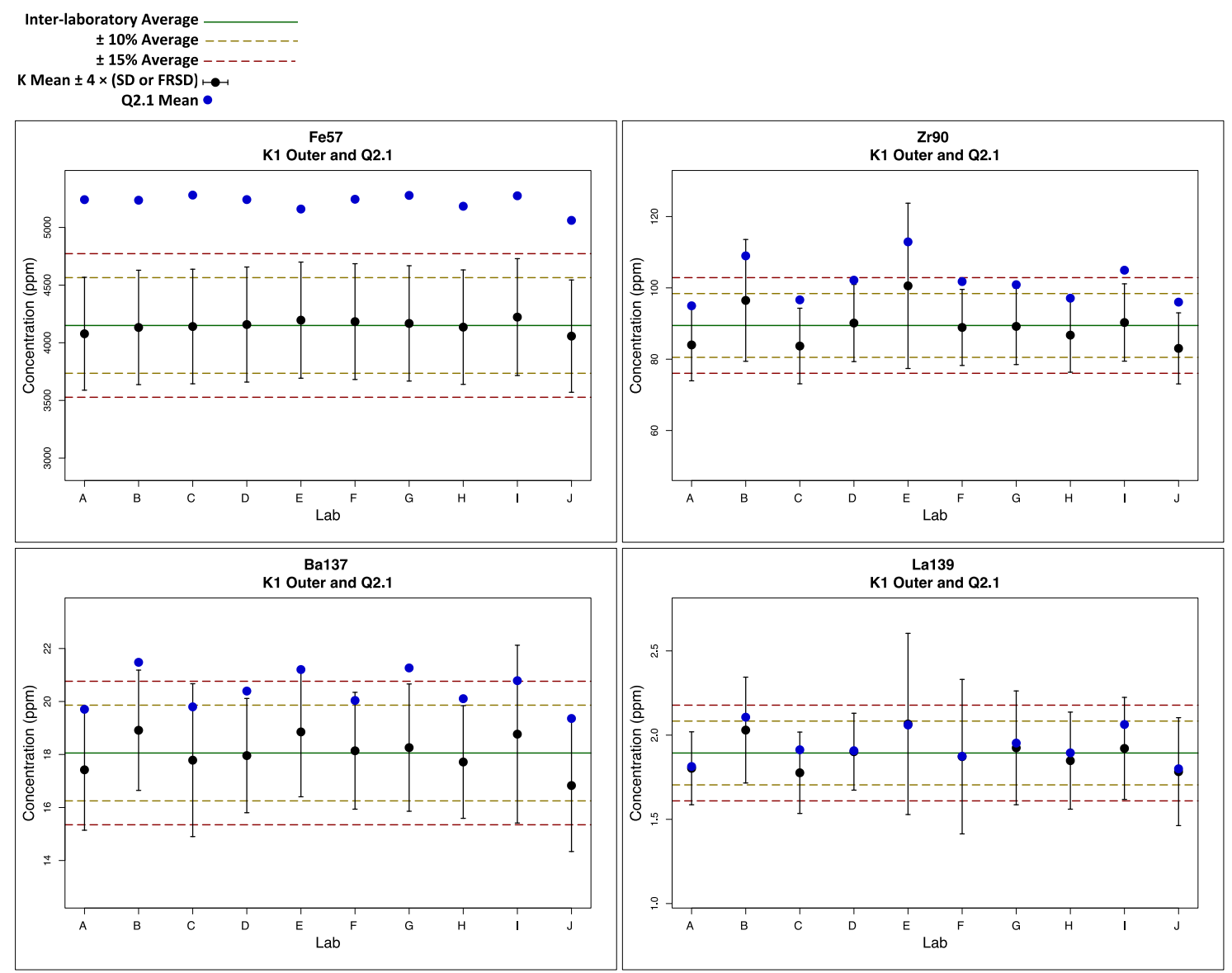

Figure 49 - ASTM E2927 comparison of K1 outer and Q2.1

10.4.2 Interpretation of the Third Inter-Laboratory Exercise Results

Using the verbal scale that was sent out, only seven laboratories sent back a response. For the comparison of K1 outer with all Q1 fragments, three of the labs said the results were a "Level 2: Association with highly discriminating characteristics." The remaining four labs classified the results as "Level 3: Association with discriminating characteristics."

\subsubsection{Frequency for Third Inter-Laboratory Test}

$\mathrm{K} 1$ for this exercise originated from sample 21. As previously mentioned, the outer panes of samples 21,45 , and 78 were found to be indistinguishable in the database, 
however for the second exercise, differences were seen between the data submitted by the labs for sample 78 and the database data for samples 21 and 45 . The data for K1 outer for this exercise from all labs were indistinguishable with the outer pane of samples 21 and 45 in the database. For eight of the labs, K1 outer was indistinguishable with the outer pane of sample 78. For the other two labs, this sample was distinguished by the same element, ${ }^{137} \mathrm{Ba}$. When examining how labs' data for sample 21 compares to the database's 21, Figure 50, the concentration for ${ }^{137} \mathrm{Ba}$ was found to be very similar, unlike what was seen for ${ }^{208} \mathrm{~Pb}$ in the second exercise. Therefore, the differences between the lab's data for $\mathrm{K} 1$ and sample 78 in the database are most likely caused by the "fall off the cliff" effect and not by failing to account for the inter-day variation of ${ }^{137} \mathrm{Ba}$ in the database. For K1 outer the frequency is $3 / 703$ for Labs B, C, D, E, F, G, and I. The frequency is $2 / 703$ for Labs A and J. 


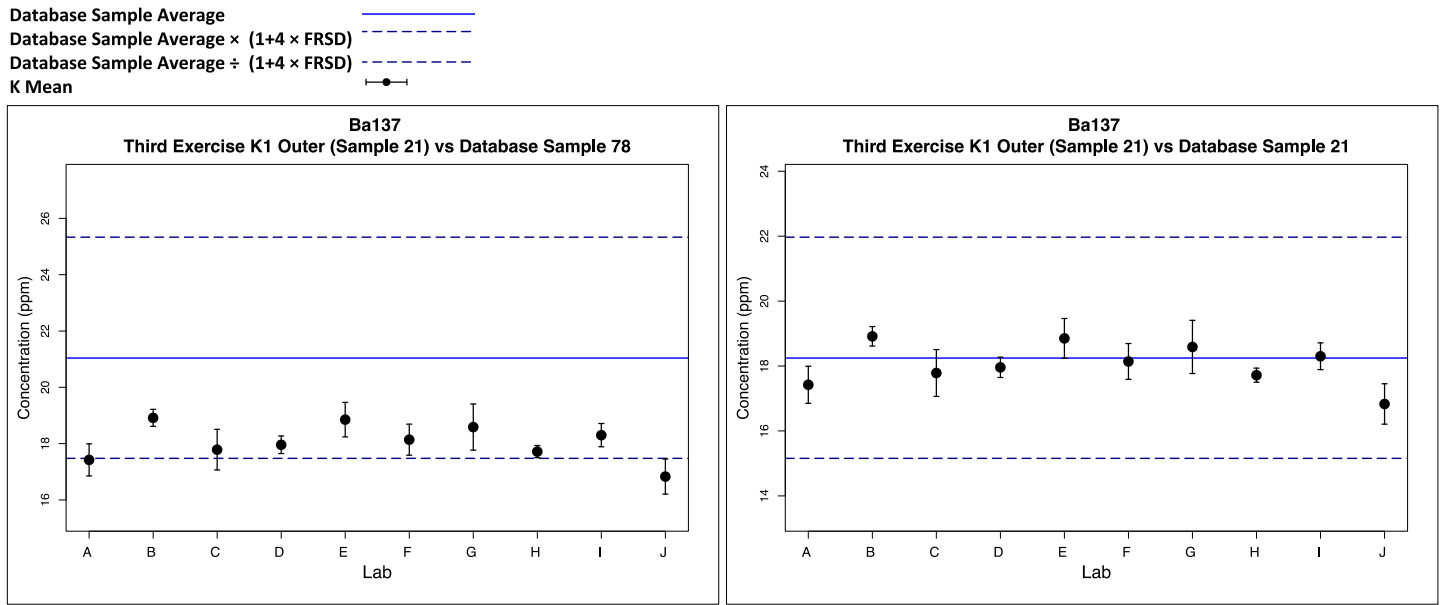

Figure 50 - Comparison of K1 outer to sample 78 and 21 in the database

10.5 Conclusions

The performance of ten (10) forensic laboratories participating in three (3) interlaboratory exercises that assess the use of ASTM 2927-16e1 for the LA-ICP-MS analysis and comparison of glass evidence from mock case scenarios was evaluated. The rate of misleading evidence (ROME) for these sets of blind scenarios when the participating laboratories were asked to compare the glass samples (K vs. Q) and report on their findings as they would in an actual case was also determined. Three different databases were used as background populations in order to calculate frequency of occurrence for the case scenarios that were distributed as part of the inter-laboratory exercises. The results of the inter-laboratory exercises suggest that, the participating laboratories correctly associate glass samples originating from the same source $100 \%$ of the time (total of 57 comparisons requested) and correctly discriminate glass samples from different sources $100 \%$ of the time (total of 167 comparisons requested throughout the three exercises). This error rate does not include the results of the first inter-laboratory exercise because each lab used a different match criterion rather than the standardized ASTM match criterion. The random match probability of glass samples known to 
originate from two different glass sources was found to be $\sim 0.1 \%$ and is in agreement with previously reported values by other research groups. 


\section{CHAPTER 11. Refractive Index Study}

For the first inter-laboratory study, K1 outer and Q1 originated from very similar vehicles (same make, model, year and VINs $<5000$ apart). Elemental analysis using LAICP-MS was not able to detect differences in these glasses, however, the labs that included RI as part of their analytical scheme were able to differentiate these glasses. Today, some forensic labs are considering removing RI from their analytical scheme. In this chapter, the usefulness of RI to distinguish cars with indistinguishable elemental profiles was tested.

\subsection{Sample Set}

All samples came from the FIU M\&M vehicle glass collection. The samples were selected in pairs of vehicles with the same make, same year, and elemental profiles that were indistinguishable from each other. The sample pairs can be seen in Table 25. Care was taken to select some glass samples that were indistinguishable with multiple glasses: sample 002 , sample 018 , sample 024 . A total of 31 samples were sent which resulted in 22 pairs. A full thickness glass fragment for each sample was selected, wrapped in weighing paper, and placed in a labeled white pill box with only the sample number on it. These samples were sent off to another lab for the RI measurements.

\subsection{Refractive Index Measurements}

The refractive index measurements were conducted at West Virginia University. The true identity of each sample was not disclosed. The lab was asked to take at least 4 replicate measurements using ASTM E1967 [63] and report the replicate measurements for each sample. 


\subsection{Comparing Refractive Index}

There is no standard for the forensic comparison of refractive index, however most participants in the GIWG use a comparison interval created by the mean of the $\mathrm{K} \pm$ $3 \times$ SD. Some state a minimum SD of 0.00003 or 0.000033 . One participant used a comparison criterion of the mean $\mathrm{K} \pm 0.0001$. The SD was not taken into account at all. Regardless of the comparison interval, if the Q mean falls within the comparison interval, the samples are indistinguishable.

To compare the RI of these samples, the comparison interval of $\mathrm{K} \pm 3 \times \mathrm{SD}$ with a minimum SD of 0.00003 was used.

\subsection{Results}

The results of the RI comparisons can be seen in the last column of Table 25 . Sample 002 inner and sample 005 outer were analyzed twice. Both duplicates were used in the comparison. Since the comparison interval changes depending on which sample is selected as the $\mathrm{K}$ and taking into consideration that two samples, which are included in 3 of the pairs were ran in duplicate, there were 50 of these challenging comparisons. There were 12 comparisons that were distinguished by RI. However, there were instances where a comparison pair was distinguished when one sample was $\mathrm{K}$ and indistinguishable when

the other was selected as the K. The duplicates also provided mixed results. The results for these types of comparison pairs were therefore called inconclusive. In total, there were 3 pairs (6 comparisons) of vehicles that were distinguished by RI regardless of which sample was used as the K. 
These 3 pairs of vehicles were all same make, model, and year. The VINs are very similar. Most of these samples did not have a readable window marking, so the manufacturer or the glass and date the glass was manufactured is unknown. Since most vehicle manufacturers have an annual contract with a glass manufacturer, it is assumed that the pairs selected for this study were all produced by the same manufacturer around the same time. It could be that during the process of turning the float glass sheets into laminated windshields, the heating process slightly alters the refractive index. If different enough, this difference in thermal history could be used to distinguish very similar glass samples from each other.

If all possible pairs are considered and not just the challenging ones, there is a total of 1056 comparison pairs. Of these 1056 comparisons, 950 were distinguished by RI $(\sim 90 \%)$.

This study shows that refractive index is still a useful tool in forensic labs. All the samples selected were challenging cases in which sensitive elemental tools were unable to detect statistical differences. The fact that some of these challenging cases were distinguished by RI shows that this technique still is useful in forensic laboratories. 
Table 25 - Sample pairs that were found to be indistinguishable with LA-ICP-MS analysis used for refractive index study

\begin{tabular}{|c|c|c|c|c|c|c|}
\hline & Pairs & Year & Make & Model & VIN & RI Results \\
\hline 1 & $\begin{array}{l}002 \text { Inner } \\
068 \text { Outer }\end{array}$ & $\begin{array}{l}2013 \\
2013\end{array}$ & $\begin{array}{l}\text { Honda } \\
\text { Honda }\end{array}$ & $\begin{array}{l}\text { Civic } \\
\text { Civic }\end{array}$ & $\begin{array}{l}\text { 2HGFG3B54DH500424 } \\
\text { 2HGFG3B59DH501522 }\end{array}$ & Indistinguishable \\
\hline 2 & $\begin{array}{l}002 \text { Inner } \\
069 \text { Outer }\end{array}$ & $\begin{array}{l}2013 \\
2013 \\
\end{array}$ & $\begin{array}{l}\text { Honda } \\
\text { Honda }\end{array}$ & $\begin{array}{l}\text { Civic } \\
\text { Civic }\end{array}$ & $\begin{array}{l}\text { 2HGFG3B54DH500424 } \\
\text { 2HGFB2F50DH504344 }\end{array}$ & Inconclusive \\
\hline 3 & $\begin{array}{l}005 \text { Outer } \\
080 \text { Inner }\end{array}$ & $\begin{array}{l}2016 \\
2016\end{array}$ & $\begin{array}{l}\text { Ford } \\
\text { Ford }\end{array}$ & $\begin{array}{l}\text { Mustang } \\
\text { F-150 }\end{array}$ & $\begin{array}{l}\text { 1FA6P8CF7G5224485 } \\
\text { 1FTEX1CF1GFA20367 }\end{array}$ & Inconclusive \\
\hline 4 & $\begin{array}{l}010 \text { Inner } \\
090 \text { Inner }\end{array}$ & $\begin{array}{l}2015 \\
2015\end{array}$ & $\begin{array}{l}\mathrm{Kia} \\
\mathrm{Kia}\end{array}$ & $\begin{array}{l}\text { Sedona } \\
\text { Sedona }\end{array}$ & $\begin{array}{l}\text { KNDMC5C16F6019328 } \\
\text { KNDMC5C14F6018761 }\end{array}$ & Indistinguishable \\
\hline 5 & $\begin{array}{l}012 \text { Outer } \\
\text { 020 Inner }\end{array}$ & $\begin{array}{l}2013 \\
2013\end{array}$ & $\begin{array}{l}\text { Honda } \\
\text { Honda }\end{array}$ & $\begin{array}{l}\text { Accord } \\
\text { Accord }\end{array}$ & $\begin{array}{l}\text { 1HGCT1B38DA000184 } \\
\text { 1HGCR2F37DA011773 }\end{array}$ & Indistinguishable \\
\hline 6 & $\begin{array}{l}018 \text { Inner } \\
\text { 024 Inner }\end{array}$ & $\begin{array}{l}2014 \\
2014\end{array}$ & $\begin{array}{l}\text { Mitsubishi } \\
\text { Mitsubishi }\end{array}$ & $\begin{array}{l}\text { Mirage } \\
\text { Mirage }\end{array}$ & $\begin{array}{l}\text { ML32A3НJ6EH003209 } \\
\text { ML32A3HJ4EH003547 }\end{array}$ & Indistinguishable \\
\hline 7 & $\begin{array}{l}018 \text { Inner } \\
106 \text { Outer }\end{array}$ & $\begin{array}{l}2014 \\
2014 \\
\end{array}$ & $\begin{array}{l}\text { Mitsubishi } \\
\text { Mitsubishi }\end{array}$ & $\begin{array}{l}\text { Mirage } \\
\text { Mirage }\end{array}$ & $\begin{array}{l}\text { ML32A3HJ6EH003209 } \\
\text { ML32A3HJ1EH003425 }\end{array}$ & Indistinguishable \\
\hline 8 & $\begin{array}{l}\text { 024 Inner } \\
106 \text { Outer }\end{array}$ & $\begin{array}{l}2014 \\
2014\end{array}$ & $\begin{array}{l}\text { Mitsubishi } \\
\text { Mitsubishi }\end{array}$ & $\begin{array}{l}\text { Mirage } \\
\text { Mirage }\end{array}$ & $\begin{array}{l}\text { ML32A3HJ4EH003547 } \\
\text { ML32A3HJ1EH003425 }\end{array}$ & Indistinguishable \\
\hline 9 & $\begin{array}{l}\text { 025 Inner } \\
041 \text { Inner }\end{array}$ & $\begin{array}{l}2010 \\
2010 \\
\end{array}$ & $\begin{array}{l}\text { Kia } \\
\text { Kia } \\
\end{array}$ & $\begin{array}{l}\text { Forte } \\
\text { Forte }\end{array}$ & $\begin{array}{l}\text { KNAFW4A34A5148033 } \\
\text { KNAFU4A29A5143195 }\end{array}$ & Indistinguishable \\
\hline 10 & $\begin{array}{l}025 \text { Inner } \\
097 \text { Inner }\end{array}$ & $\begin{array}{l}2010 \\
2010\end{array}$ & $\begin{array}{l}\mathrm{Kia} \\
\mathrm{Kia}\end{array}$ & $\begin{array}{l}\text { Forte } \\
\text { Forte }\end{array}$ & $\begin{array}{l}\text { KNAFW4A34A5148033 } \\
\text { KNAFU4A23A5809774 }\end{array}$ & Indistinguishable \\
\hline 11 & $\begin{array}{l}028 \text { Outer } \\
047 \text { Outer }\end{array}$ & $\begin{array}{l}2012 \\
2012\end{array}$ & $\begin{array}{l}\text { Honda } \\
\text { Honda }\end{array}$ & $\begin{array}{l}\text { Civic } \\
\text { Pilot }\end{array}$ & $\begin{array}{l}\text { 2HGFB2F52CH300384 } \\
\text { 5FNYF4H41CB001552 }\end{array}$ & Indistinguishable \\
\hline 12 & $\begin{array}{l}\text { 029 Inner } \\
\text { 053 Inner }\end{array}$ & $\begin{array}{l}2016 \\
2016 \\
\end{array}$ & $\begin{array}{l}\text { Toyota } \\
\text { Toyota }\end{array}$ & $\begin{array}{l}\text { Tundra } \\
\text { Tundra }\end{array}$ & $\begin{array}{l}\text { 5TFEY5F18GX197447 } \\
\text { 5TFEY5F14GX199602 }\end{array}$ & Indistinguishable \\
\hline & $\begin{array}{l}029 \text { Outer } \\
\text { 053 Inner }\end{array}$ & $\begin{array}{l}2016 \\
2016 \\
\end{array}$ & $\begin{array}{l}\text { Toyota } \\
\text { Toyota }\end{array}$ & $\begin{array}{l}\text { Tundra } \\
\text { Tundra }\end{array}$ & $\begin{array}{l}\text { 5TFEY5F18GX197447 } \\
\text { 5TFEY5F14GX199602 }\end{array}$ & Indistinguishable \\
\hline 13 & $\begin{array}{l}039 \text { Outer } \\
055 \text { Outer }\end{array}$ & $\begin{array}{l}2016 \\
2016\end{array}$ & $\begin{array}{l}\text { Mazda } \\
\text { Mazda }\end{array}$ & $\begin{array}{l}\mathrm{CX}-3 \\
\mathrm{CX}-3\end{array}$ & $\begin{array}{l}\text { JM1DKFB72G0129070 } \\
\text { JM1DKFC79G0136435 }\end{array}$ & Indistinguishable \\
\hline 14 & $\begin{array}{l}041 \text { Inner } \\
097 \text { Inner }\end{array}$ & $\begin{array}{l}2010 \\
2010\end{array}$ & $\begin{array}{l}\text { Kia } \\
\text { Kia }\end{array}$ & $\begin{array}{l}\text { Forte } \\
\text { Forte }\end{array}$ & $\begin{array}{l}\text { KNAFU4A29A5143195 } \\
\text { KNAFU4A23A5809774 }\end{array}$ & Indistinguishable \\
\hline 15 & $\begin{array}{l}042 \text { Inner } \\
185 \text { Inner }\end{array}$ & $\begin{array}{l}2014 \\
2014\end{array}$ & $\begin{array}{l}\text { BMW } \\
\text { BMW }\end{array}$ & $\begin{array}{l}2 \text { Series } \\
2 \text { Series }\end{array}$ & $\begin{array}{l}\text { WBA1F5C58EVV98871 } \\
\text { WBA1F5C59EVV98894 }\end{array}$ & Inconclusive \\
\hline 16 & $\begin{array}{l}044 \text { Inner } \\
129 \text { Inner }\end{array}$ & $\begin{array}{l}2016 \\
2016 \\
\end{array}$ & $\begin{array}{l}\text { Nissan } \\
\text { Nissan }\end{array}$ & $\begin{array}{l}\text { Maxima } \\
\text { Maxima }\end{array}$ & $\begin{array}{l}\text { 1N4AA6AP7GC380842 } \\
\text { 1N4AA6AP3GC378439 }\end{array}$ & Distinguished \\
\hline 17 & $\begin{array}{l}\text { 050 Inner } \\
149 \text { Inner }\end{array}$ & $\begin{array}{l}2014 \\
2014 \\
\end{array}$ & $\begin{array}{l}\text { Hyundai } \\
\text { Hyundai }\end{array}$ & $\begin{array}{l}\text { Veloster } \\
\text { Veloster }\end{array}$ & $\begin{array}{l}\text { KMHTC6AD8EU195530 } \\
\text { KMHTC6AD9EU195701 }\end{array}$ & Indistinguishable \\
\hline 18 & $\begin{array}{l}065 \text { Outer } \\
066 \text { Outer }\end{array}$ & $\begin{array}{l}2009 \\
2009 \\
\end{array}$ & $\begin{array}{l}\text { Mitsubishi } \\
\text { Mitsubishi }\end{array}$ & $\begin{array}{l}\text { Galant } \\
\text { Galant }\end{array}$ & $\begin{array}{l}\text { 4A3AB36F49E015450 } \\
\text { 4A3AB36F39E029145 }\end{array}$ & Indistinguishable \\
\hline 19 & $\begin{array}{l}065 \text { Outer } \\
110 \text { Outer }\end{array}$ & $\begin{array}{l}2009 \\
2009\end{array}$ & $\begin{array}{l}\text { Mitsubishi } \\
\text { Mitsubishi }\end{array}$ & $\begin{array}{l}\text { Galant } \\
\text { Galant }\end{array}$ & $\begin{array}{l}\text { 4A3AB36F49E015450 } \\
\text { 4A3AB36F39E024088 }\end{array}$ & Indistinguishable \\
\hline 20 & $\begin{array}{l}066 \text { Outer } \\
110 \text { Outer }\end{array}$ & $\begin{array}{l}2009 \\
2009\end{array}$ & $\begin{array}{l}\text { Mitsubishi } \\
\text { Mitsubishi }\end{array}$ & $\begin{array}{l}\text { Galant } \\
\text { Galant }\end{array}$ & $\begin{array}{l}\text { 4A3AB36F39E029145 } \\
\text { 4A3AB36F39E024088 }\end{array}$ & Distinguished \\
\hline 21 & $\begin{array}{l}068 \text { Outer } \\
069 \text { Outer }\end{array}$ & $\begin{array}{l}2013 \\
2013\end{array}$ & $\begin{array}{l}\text { Honda } \\
\text { Honda }\end{array}$ & $\begin{array}{l}\text { Civic } \\
\text { Civic }\end{array}$ & $\begin{array}{l}\text { 2HGFG3B59DH501522 } \\
\text { 2HGFB2F50DH504344 }\end{array}$ & Distinguished \\
\hline
\end{tabular}




\section{CHAPTER 12. Analysis \& Interpretation of Glass Conclusions}

The main goals of this project were to collect existing elemental glass databases and create of a new vehicle database, evaluate the current ASTM guidelines for the analysis and comparison of glass evidence, and asses the use of different statistical models for the objective and quantitative interpretation of glass evidence using a large user community of glass examiners named the Glass Interpretation Working Group (GIWG).

Five databases were collected and a new vehicle glass database consisting of windshield glass from 210 different vehicles was created using LA-ICP-MS. The new vehicle database was used to determine a comparison criterion for comparing database samples to each other and to casework samples. This comparison criterion needed to incorporate inter-day variation since most databases are generated over a couple of years. The comparison criterion that was found to correctly associate duplicate pairs $92 \%$ (48/52 pairs) of the time was a comparison interval for each element where the upper limit was sample 1 mean $\times(1+4 \times$ FRSD $)$ and the lower limit was sample 1 mean $\div(1+4 \times$ FRSD). If the mean of sample 2 fell within the comparison interval for all elements, the samples were indistinguishable. This comparison interval was selected because the end result, indistinguishable or distinguished, is the same regardless of which sample was selected as sample 1.The FRSDs were based off of the inter-day variability of two control glasses and the variability of each element in glasses that originate from the same source and different sources.

Using this comparison criteria, the random match probability (false inclusion) of the collected and created databases was found to range from $0.06-0.2 \%$. The $0.2 \%$ was 
for the FIU vehicle database. This database contains a smaller population variation since it has many cars with similar years and makes. Thus it is highly likely to contain many glasses that were produced at the same manufacturer around the same time. When similar vehicles are removed, the random match probability drops to $\sim 0.1 \%$. Even though each tested database represents a snapshot of a different place and time, the probability of finding two glasses from different sources with the same elemental profile was found to consistently be $\sim 0.1 \%$. This is a useful observation that should be used to place a higher value on LA-ICP-MS elemental glass evidence.

This comparison criterion was also used to calculate the frequency of a specific elemental profile in the inter-laboratory trials. The FIU vehicle glass database and a donated casework database were used in the frequency calculations. The use of the different databases provided different frequencies. All samples used in the interlaboratory study were part of the FIU database, thus labs should find their sample to have the same elemental profile as at least one sample in the database, its duplicate. When using the FIU database, which contains glass from very similar vehicles, it was not surprising to discover that some of the samples had a frequency greater than $1 / 333$. However when the casework database was used, only one sample was found to be similar to one in the database. This sample was the Q2 in the third inter-laboratory study. It is advised to only calculate a frequency for a specific glass sample when there is a large database to work with. One forensic examiner said to only calculate a frequency when your database is greater than 4000 samples. Therefore, the frequency calculations are only used here to see if other laboratories can produce data for a sample that is found to be indistinguishable with the same sample in a database created by another user. If labs 
are able to correctly associate their data with the data from other labs, it could mean that a single common glass database can be created. For the first inter-laboratory study, there were differences in the frequency of data from labs that used a different calibration strategy than the only used to create the database. The different calibration techniques caused a large variation in the concentrations determined for $\mathrm{Mg}, \mathrm{Fe}, \mathrm{K}$, and $\mathrm{Ti}$. However, labs that calibrated their data the same way as the database found similar results. In the second inter-laboratory study, all participants were told to use single-point calibration with FGS2 as the calibrator. This improved the results, but some laboratories had problematic elements, which differed from the other participants. These laboratories were not able to correctly associate their sample to its duplicate in the database. By the third inter- laboratory study, most of the problematic elements were fixed. The data from all of the participants were correctly associated to the duplicate in the database, and similar frequencies were seen when using the FIU vehicle database. Though these results indicate that it may be possible to combine databases intro a central repository, it may not be appropriate for a German casework database to be combined with a database of windshield glass collected in the United States. More research needs to be done. However, the combined database was included as an exercise in this study in order to test how the random match probability and frequency changes when using glass databases of different sizes and comprised of diverse samples.

When evaluating the ASTM E2927 comparison criterion for comparing K and Q samples ran on the same day, the results of the inter-laboratory exercises suggest that the participating laboratories correctly associate glass samples originating from the same source $100 \%$ of the time (total of 57 comparisons requested) and correctly discriminate 
glass samples from different sources $100 \%$ of the time (total of 167 comparisons requested throughout the three exercises). This error rate does not include the results of the first inter-laboratory exercise because each lab used a different match criterion rather than the standardized ASTM match criterion.

The inter-laboratory studies also showed how labs using a verbal scale differ in the significance they place on the same evidence. For example when the participants were told to interpret their results considering only the LA-ICP-MS analysis, some labs said that the evidence strongly suggests the $\mathrm{K}$ and Q originated from the same source while others said very strongly suggests. Even when all labs were given the same K and Q samples, found similar concentrations for the elements within these samples, and were given the same verbal scale to use and told to only consider LA-ICP-MS, forensic glass examiners still differed in what level they would place the results in. Half of the forensic glass examiners consider LA-ICP-MS and highly discriminating technique while the other half consider it to be only a discriminating technique.

My recommendations for future studies would be to see how the random match probability changes when eliminating problematic elements such as $\mathrm{Pb}$. This in one element that was discriminating some of the lab's data from the duplicate in the database. It also may be possible to combine NIST612 and FGS2 calibrated databases together with the removal of the elements that were found to differ with calibration technique: $\mathrm{K}, \mathrm{Mg}$, $\mathrm{Fe}, \mathrm{Ti}$, and Mn. Also, other forensic glass examiners suggest that a database of at least 4000 samples should be used to calculate the frequency of a specific glass. Removing problematic elements and combining databases would result in a database of $\sim 2500$ samples. This new combined database could be furthers tested to see if any of the inter- 
laboratory samples are found to have a similar profile to glasses in other databases. Another recommendation would be to further test how well refractive index can pick up differences in the thermal history of glass. This tool could be used to distinguish vehicle glass produced at the same manufacturing plant around the same time since the glass from these plants needs to be heated in molds to form the vehicle windshields, and thus have a slightly different thermal history. Lastly, since the new comparison criterion needed to incorporate inter-day variation, there were some samples from different vehicles that were found to be indistinguishable. If these samples would have been analyzed on the same day and compared with the ASTM E2927 comparison criterion with a FRSD of 3\%, would they still be indistinguishable or are there differences that can be detected. 


\section{CHAPTER 13. Overall Conclusions}

The first study focused on the development of a laser based method for the elemental analysis of solid milk powder. A variety of sample preparations and calibration strategies were tested and evaluated using a reference milk powder IAEA-153. These sample preparations included: spreading milk powder on tape, dried discs, dried spots, and pellets. The calibration strategies tested included external calibration curves, standard addition curves, and single point calibration. The best sample preparation method was a standard addition method that involved creating pellets. This method yields accurate elemental concentrations for $\mathrm{Na}, \mathrm{Mg}, \mathrm{Ca}, \mathrm{Rb}$, and $\mathrm{Sr}$ without the need for acid digestion. Precision of $10 \%$ RSD or better and a bias of $10 \%$ were achieved for most elements.

The accuracy of this method was further evaluated by comparing the LA-ICP-MS results of 13 samples to those obtained from a primary technique, $k_{0}$-INAA. The comparison of LA-ICP-MS to $k_{0}$-INAA showed overlap of the $95 \%$ confidence intervals for all comparison samples. Thus showing that the LA-ICP-MS method performs well for a variety of different milk samples.

As a preliminary study on combining datasets from different labs, five laboratories were asked to analyze IAEA-153 and submit their data. $\mathrm{Ca}$ and $\mathrm{Zn}$ were problematic elements and their concentration differed between participants and produced a high bias for some laboratories. Thus, these elements were excluded from the menu. $\mathrm{Na}, \mathrm{Mg}, \mathrm{Sr}$, and $\mathrm{Rb}$ all performed well between the participants, which suggests that database creation is a viable option for select elements.

The data for 68 authentic milk powder samples representing five different countries (Argentina, Russia, Singapore, Slovenia, and the United States) was collected 
and used as a preliminary database. The data submitted was limited to the following elements: $\mathrm{Na}, \mathrm{Mg}, \mathrm{Rb}$, and Sr. Principle component analysis (PCA) showed different groupings for the United States, Argentina, Singapore, and Slovenia. However, the collected data represented a few localized areas and does not account for the total variation within every country. Samples collected from a large geographic area within Russia produces an expected large variation in the elemental profiles and therefore the limited element menu was not able to distinguish the Russian milk samples from those for Argentina, the United States, and Slovenia. The goal of being able to determine if commercial milk could have originated from a specific country has not been met. However, the few samples from Singapore appear to be very different from the samples from the drying facilities in the United States and Slovenia, and from the samples from Russia. If milk produced in Singapore was labeled as being made in the Russia, the elemental profile of that milk would not line up with that the current profile found in southern Russia. More samples would need be analyzed for each county to better account for the within country variation. The addition of isotope ratios, additional trace elements, or another discriminating factor may help in further distinguishing one country from another thus making it easier to determine if an unknown milk could have originated from a specific country.

The second study focused on the use of trace elemental databases for the objective interpretation of forensic glass evidence. The main goals of this study were to collect existing elemental glass databases and create a new vehicle glass database, evaluate the current ASTM guidelines for the analysis and comparison of glass evidence, and assess the use of different statistical models for the objective and quantitative interpretation of 
glass evidence using a large user community of glass examiners named the Glass Interpretation Working Group (GIWG).

Five glass databases were collected and a new vehicle glass database composed of 420 vehicle windshield samples collected from 210 different vehicles representing manufacturing dates between 2004-2017 and 26 vehicle manufacturers was created.

The new database, and data from the EAWG and GIWG were used to evaluate the ASTM E2927 comparison criterion and test the performance of the equivalence test. The inter-laboratory data from the EAWG contains some glass comparisons that are examples of worst-case scenarios (produced at the same manufacturing plant only weeks apart). Using this data, the equivalence test performed very poorly with a false exclusion error rate that ranged from $37-100 \%$ depending on how theta was calculated vs the false exclusion error rate of $0-28 \%$ for the ASTM E2927 and ASTM E2330 comparison criterion. The number of false inclusions for the equivalence test was also larger than the error rate for the ASTM comparison criterion (0-12\% vs 0-5\%).

Using the new FIU vehicle database the false exclusion rate of the ASTM E2927 comparison criterion with a FRSD of $3 \%$ for all elements was calculated. Fragments were split up as $\mathrm{K}$ and $\mathrm{Q}$ samples and compared to each other. The average false exclusion error rate was found to be $1.7 \%$ with some elements such as $\mathrm{Pb}$ flagged as problematic. ASTM E2927 states that a minimum FRSD of at least 3\% should be used. Therefore, it is possible to use a larger FRSD for elements that have been shown to vary between fragments. Each lab prior to performing casework should validate their FRSDs and element menu. 
Also the data from second and third inter-laboratory studies of the GIWG were used to assess the performance of ASTM E2927. The results showed that even with a FRSD of $3 \%$ for all elements, all laboratories were able to correctly associate samples that originated from the same source (total of 57 comparisons requested) and exclude samples that originated from different sources (total of 167 comparisons requested throughout the exercises) for all comparisons. This error rate does not include the results of the first inter-laboratory exercise because each lab used a different match criterion rather than the standardized ASTM match criterion.

The ASTM E2927 comparison criterion performs great when comparing a K and Q that were analyzed on the same day. However, this comparison criterion is not designed to compare samples that were analyzed days, months, or years apart. Therefore, the new vehicle database was used to determine a comparison criterion for comparing database samples to each other and to casework samples. Daily quality control samples as well as 52 duplicate samples analyzed months apart were assessed to determine the interday variability of the database and evaluate different approaches to incorporate the interday variation into a comparison criterion.

The comparison criterion that was found to correctly associate the duplicate pairs $98 \%$ (51/52 pairs) of the time was a comparison interval for each element where the upper limit was sample 1 mean $\times(1+4 \times$ FRSD $)$ and the lower limit was sample 1 mean $\div(1+4 \times$ FRSD). If the mean of sample 2 fell within the comparison interval for all elements, the samples were indistinguishable. This comparison interval was selected because the end result, indistinguishable or distinguished, is the same regardless of which sample was selected as sample 1.The FRSDs were based off of the inter-day variability of 
two control glasses and the variability of each element in glasses that originate from the same source and different sources. There were some issues with improper calibration of the detector for $\mathrm{Ce}$, so the seven samples in the new database with a concentration above 2000 ppm were flagged and the Ce of these samples was not used for the comparisons.

Using this new comparison criteria, the random match probability (false inclusion) of the collected and created databases was found to range from $0.06-0.2 \%$. The $0.2 \%$ was for the FIU vehicle database. This database contains a smaller population variation since it has many cars with similar years and makes. Thus it is highly likely to contain many glasses that were produced at the same manufacturer around the same time. Removing the indistinguishable pairs that are known to come from the same car (inner and outer panes) and cars that are the same make and year, the false inclusion error rate decreased to $0.1 \%$. The fact that most of the collected databases (the exception being a very small database of $\sim 40$ samples) that represent different times and locations have a random match probability of $\sim 0.1 \%$ should be noted. This means that 1 out of 1000 comparisons will result in a false inclusions. This random match probability gives an indication of how powerful a tool LA-ICP-MS is for glass analysis. Also, this random match probability could even be reduced further if forensic laboratories incorporate thickness measurements and/or RI as part of their analytical scheme. The usefulness of RI to distinguish cars with indistinguishable elemental profiles was tested. A small sample set of samples in which elemental analysis using LA-ICP-MS was not able to detect differences was selected. Since there is currently no standard for the forensic comparison of refractive index, the comparison criterion used by some of the participants in the GIWG was used to compare samples. This criterion was a comparison interval created by 
the mean of the $\mathrm{K} \pm 3 \times \mathrm{SD}$. Some participants state a minimum SD of 0.00003 . If the $\mathrm{Q}$ mean falls within the comparison interval, the samples are indistinguishable. Though there were some mixed results in which switching the K and Q samples caused the pair to go from indistinguishable to distinguished, there were some pairs that were distinguished both ways by RI. This small study shows that refractive index is still a useful tool in forensic labs. All the samples selected were challenging cases in which sensitive elemental tools were unable to detect statistical differences.

This comparison criterion was also used to calculate the frequency of a specific elemental profile in the GIWG inter-laboratory trials. The new FIU vehicle glass database and a donated casework database were used in the frequency calculations. The use of the different databases provided different frequencies. All samples used in the interlaboratory study were part of the FIU database, thus labs should find their sample to have the same elemental profile as at least one sample in the database, its duplicate. When using the FIU database, which contains glass from very similar vehicles, it was not surprising to discover that some of the samples had a frequency greater than $1 / 333$. However when the casework database was used, only one sample was found to be similar to one in the database. This sample was the Q2 in the third inter-laboratory study. It is advised to only calculate a frequency for a specific glass sample when there is a large database to work with. One forensic examiner said to only calculate a frequency when your database is greater than 4000 samples. Therefore, the frequency calculations are only used here to see if other laboratories can produce data for a sample that is found to be indistinguishable with the same sample in a database created by another user. If labs 
are able to correctly associate their data with the data from other labs, it could mean that a single common glass database can be created.

For the first inter-laboratory study, there were differences in the frequency of data from labs that used a different calibration strategy than the database. The different calibration technique caused a large variation in the concentrations determined for $\mathrm{Mg}$, $\mathrm{Fe}, \mathrm{K}$, and Ti. However, labs that calibrated their data the same way as the database found similar results. In the second inter-laboratory study, all participants were told to use single-point calibration with FGS2 as the calibrator. This improved the results, but some laboratories had problematic elements, which differed from the other participants. These laboratories were not able to correctly associate their sample to its duplicate in the database. By the third inter- laboratory study, most of the problematic elements were fixed. The data from all of the participants were correctly associated to the duplicate in the database, and similar frequencies were seen when using the FIU vehicle database. Though these results indicate that it may be possible to combine databases intro a central repository, it may not be appropriate for a European casework database to be combined with a database of windshield glass collected in the United States. More research needs to be done. However, the combined database was included as an exercise in this study in order to test how the random match probability and frequency changes when using glass databases of different sizes and comprised of diverse samples.

The inter-laboratory study indicates that the majority of forensic laboratories use a comparison criterion to compare a $\mathrm{K}$ and $\mathrm{Q}$ sample. Of all participating laboratories, two use a database to calculate a frequency and/or the random match probability and only one uses a likelihood ratio (score) for casework. Many of the labs used their own verbal scale, 
which sometimes resulted in different strengths for the evidence. Even when a generic verbal scale was supplied to the participants in the third inter-laboratory study, differences were still seen. Half of the respondents said that elemental analysis using LAICP-MS for the samples in the third study were highly discriminating while the other half said it was only discriminating. Seven forensic laboratories responded to a survey and, when asked what interpretation tools are appropriate for LA-ICP-MS analysis of glass (select all that apply), four participants selected a verbal scale, four selected a frequency and/or random match probability, two selected a LR, and three selected a match criterion combined with a LR. The results of this research suggest that the use of databases adds additional information that can be used to assess the significance of glass comparisons.

During both studies, I had the opportunity to interact with two different groups of researchers. The group involved in the first study all came from research laboratories and were just starting to develop methods to answer their research questions, which differed by participant. Some participants were more focused on detecting organic adulterants added to milk. Others were focused on elemental analysis to detect diluted whole milk and provenancing milk to a specific region or country. The instrumentation available to each participant varied, however each participant was willing to help donate samples and run analyses for those who needed assistance answering their research questions. Besides the development of a LA-ICP-MS method, my other major contributions to this group were evaluating the quality of data for those performing elemental analysis by comparing their concentrations for IAEA-153 and IAEA-155 to the reported values and helping the ICP-MS labs work towards a common method for digestion and analysis. My contribution assisted the participants by showing them their problematic elements and the 
importance of running a daily control sample. I also talked with them about steps in their sample preparation and analysis method that may be unnecessary and adding sources of error. This group of researchers was willing to incorporate changes to enhance the accuracy and precision of their methods.

The group of researchers in the second study mostly came from forensic laboratories. The methods of analyzing glass fragments, comparing the $\mathrm{K}$ and $\mathrm{Q}$, and interpreting the results were well defined within each laboratory. Though there is a standardized method for LA-ICP-MS analysis of soda-lime glass (ASTM E2927), not all laboratories follow this method and some of these laboratories were resistant to incorporate changes to their methods. Working with this group helped me to realize the amount of work that is involved with incorporating something new into forensic laboratories. Each laboratory was comfortable with the way they were doing things and hesitant to do anything that differed from their SOP. I was able to make some progress with the participants such as showing them that the calibration technique and choice of standard reference material are important, and when all labs used FGS2 to calibrate, I was able to show the participants how they compared with the other laboratories and identified problematic elements. I also was able to introduce some additional statistical tools that could be used to aid in interpreting results. I was able to show the laboratories how their interpretation of glass evidence differed in strength from other participants and how a standardized interpretation needs to be developed and incorporated into the report for forensic glass evidence. Most of my contribution to this group was providing evidence that forensic labs around the world differed in how they were evaluating glass 
evidence and interpreting results and making small steps to introduce them to the idea of a standardized method for the evaluation and interpretation of glass evidence. 


\section{REFERENCES}

1. United States Pharmacopeia New Additions Increase Number of Records in Usp Food Fraud Database by 60 Percent, Add Seafood, Clouding Agents, and Lemon Juice as Food Vulnerable to Fraud. Food Chemicals Codex e-Newsletter, 2013. p. $1-5$.

2. Relsch, M. S., Momentum Builds to Keep Fraudulent Food Off of Store Shelves. C\&EN. 92: p. 8-13.

3. Xiu, C., Klein, K. K., Melamine in Milk Products in China: Examining the Factors That Led to Deliberate Use of the Contaminant. Food Policy, 2010. 35: p. 463-470.

4. Brans, H., The Eu's Country of Origin Labeling (Cool) Policy, in USDA Foreign Agriculture Service GAIN Report, C. Guven, Editor. 2014.

5. Purcell, D., New Milk Powder Scandal in China, in The New Zealand Herald.

6. Potocnik, D., Necemer, M., Mazej, D., Jacimovic, R., Ogrinc, N., MultiElemental Composition of Slovenian Milk: Analytical Approach and Geographical Origin Determination. Acta Imeko, 2016. 5: p. 15-21.

7. Park, Y. W., Haenlein, G. F. W., Milk and Dairy Products in Human Nutrition: Production, Composition and Health. 2013, Chichester, West Sussex, UK: John Wiley \& Sons, Ltd.

8. Bylund, G., Dairy Processing Handbook. 2003, Tetra Pak Processing Systems AB, 2003: Lund, Sweden.

9. Zamberlin, S., Antunac, N., Havranek, J., Samarzija, D., Mineral Elements in Milk and Dairy Products. Mjekarstvo, 2012. 62: p. 111-125.

10. National Research Council (US) Committee on Technological Options to Improve the Nutritional Attributes of Animal Products, Designing Foods: Animal Product Options in the Marketplace, in Factors affecting the composition of milk from dairy cows. 1998, National Academies Press (US): Washington (DC). 
11. Miciński, J., Pogorzelska, J., Beisenov, A., Aitzhanova, I., Shaikamal, G. I., Dzięgelewska-Kuzmińska, D., Miciński, B., Sobczuk-Szul, M., Basic and Mineral Composition of Colostrum from Cows in Different Ages and Calving Period. Journal of Elementology, 2016.

12. Tsioulpas, A., Grandison, A. S., Lewis, M. J., Changes in Physical Properties of Bovine Milk from the Colostrum Period to Early Lactation. Journal of Dairy Science, 2007. 90: p. 5012-5017.

13. Cerbulis, J., Farrell, H., Composition of the Milks of Dairy Cattle. Ii. Ash, Calcium, Magnesium, and Phosphorus. Journal of Dairy Science, 1976. 59: p. 589-593.

14. Dandare, S., Ezeonwumelu, I., Abubakar, M., Comparative Analysis of Nutrient Composition of Milk from Different Breeds of Cows. European Journal of Applied Engineering and Scientific Research, 2014. 3: p. 33-36.

15. Hermansen, J., Badsberg, J., Kristensen, T., Gundersen, V., Major and Trace Elements in Organically or Conventionally Produced Milk. J Dairy Res, 2005. 72: p. 362-368.

16. Gaucheron, F., Milk Minerals, Trace Elements, and Macroelements, in Milk and Dairy Products in Human Nutrition: Production, Composition, and Health. 2013, John Wiley \& Sons, Ltd.: Chichester, West Sussex, UK.

17. Patra, R. C., Swarup, D., Kumar, P., Nandi, D., Naresh, R., Ali, S. L., Milk Trace Elements in Lactating Cows Environmentally Exposed to Higher Level of Lead and Cadmium around Different Industrial Units. Science of The Total Environment, 2008. 404: p. 36-43.

18. Krelowska - Kulas, M., Lead, Cadmium, Iron, Copper and Zinc in Fresh Milk from the Selected Areas of the Cracow Region. Food / Nahrung, 1990. 34: p. 213217.

19. Coni, E., Bocca, A., Ianni, D., Caroli, S., Preliminary Evaluation of the Factors Influencing the Trace Element Content of Milk and Dairy Products. Food Chemistry, 1995. 52: p. 123-130. 
20. Herwig, N., Stephan, K., Panne, U., Pritzkow, W., Vogl, J., Multi-Element Screening in Milk and Feed by Sf-Icp-Ms. Food Chemistry, 2011. 124: p. 12231230.

21. Sola-Larrañaga, C., Navarro-Blasco, I., Chemometric Analysis of Minerals and Trace Elements in Raw Cow Milk from the Community of Navarra, Spain. Food Chemistry, 2009. 112: p. 189-196.

22. Sacco, D., Brescia, M. A., Sgaramella, A., Casiello, G., Buccolieri, A., Ogrinc, N., Sacco, A., Discrimination between Southern Italy and Foreign Milk Samples Using Spectroscopic and Analytical Data. Food Chemistry, 2009. 114: p. 15591563.

23. Tamime, A. Y., Robinson R. K, Michel, M., Microstructure of Concentrated and Dried Milk Products, in Structure of Dairy Products, A.Y. Tamime, Editor. 2008, Blackwell Publishing Ltd,: Ames, Iowa. p. 122-151.

24. Schuck, P., Condensed and Powdered Milk, in Milk and Dairy Products in Human Nutrition: Production, Composition and Health, Y.W. Park and G.F.W. Haenlein, Editors. 2013, John Wiley \& Sons, Ltd: Chichester, West Sussex, UK. p. 412-434.

25. Brescia, M., Caldarola, V., Buccolieri, G., Dell'Atti, A., Sacco, A., Chemometric Determination of the Geographical Origin of Cow Milk Using Icp-Oes Data and Isotopic Ratios. A Preliminary Study. Italian Journal of Food Science, 2003: p. 329-336.

26. McKinstry, P., Indyk, H., Kim, N. D., The Determination of Major and Minor Elements in Milk and Infant Formula by Slurry Nebulisation and Inductively Coupled Plasma - Optical Emission Spectrometry (Icp-Oes). Food Chemistry, 1999. 65: p. 245-252.

27. Ataro, A., McCrindle, R., Botha, B., McCrindle, C., Ndibewu, P., Quantification of Trace Elements in Raw Cow's Milk by Inductively Coupled Plasma Mass Spectrometry (Icp-Ms). Food Chemistry, 2008. 111: p. 243-248.

28. Hua, K., Kay, M., Indyk, H., Nutritional Element Analysis in Infant Formulas by Direct Dispersion and Inductively Coupled Plasma-Optical Emission Spectrometry. Food Chemistry, 2000. 68: p. 463-470. 
29. Nobrega, J. A., Gelinas, Y., Krushevska, A., Barnes, R. M., Direct Determination of Major and Trace Elements in Milk by Inductively Coupled Plasma Atomic Emission and Mass Spectrometry. Journal of Analytical Atomic Spectrometry, 1997. 12: p. 1243-1246.

30. Abdel-Salam, Z., Al Sharnoubi, J., Harith, M., Qualitative Evaluation of Maternal Milk and Commercial Infant Formulas Via Libs. Talanta, 2013. 115: p. 422-426.

31. Ghezelbash, M., Darbani, M., Rosta, S., Eslami Majd, A., Soltanol Kotab, M., Mosavi Azar, A., Application of Libs for Identification and Determination of Element Concentrations in Milk. Iranian Journal of Nutrition Sciences \& Food Technology, 2015. 10: p. 89-96.

32. Gilon, N., El-Haddad, J., Stankova, A., Lei, W., Ma, Q., Motto-Ros, V., Yu, J., A Matrix Effect and Accuracy Evaluation for the Determination of Elements in Milk Powder Libs and Laser Ablation/Icp-Oes Spectrometry. Analytical and bioanalytical chemistry, 2011. 401: p. 2681-2689.

33. Lei, W., El Haddad, J., Motto-Ros, V., Gilon-Delepine, N., Stankova, A., Ma, Q., Bai, X., Zheng, L., Zeng, H., Yu, J., Comparative Measurements of Mineral Elements in Milk Powders with Laser-Induced Breakdown Spectroscopy and Inductively Coupled Plasma Atomic Emission Spectroscopy. Analytical and bioanalytical chemistry, 2011. 400: p. 3303-3313.

34. Greenberg, R., Bode, P., De Nadai Fernandes, E., Neutron Activation Analysis: A Primary Method of Measurement. Spectrochimica Acta Part B: Atomic Spectroscopy, 2011. 66: p. 193-241.

35. Choppin, G., Liljenzin, J., Rydberg, J., Ekberg, C., Radiochemistry and Nuclear Chemistry. 2013: Elsevier Science.

36. Corliss, W., Neutron Activation Analysis. 1963: U.S. Atomic Energy Commission, Division of Technical Information.

37. Skoog, D., Holler, F. J., Crouch, S. R., Instrumental Analysis. 2007: CENGAGE Learning. 
38. Cherry, S., Sorenson, J., Phelps, M., Chapter 7 - Radiation Detectors, in Physics in Nuclear Medicine (Fourth Edition). 2012, W.B. Saunders: Philadelphia. p. 87106.

39. Hoffman, T., Jaćimović, R., Bay, L. J., Griboff, J., Jagodic, M., Monferrán, M., Ogrinc, N., Podkolzin, I., Wunderlin, D., Almirall, J., Development of a Method for the Elemental Analysis of Milk Powders Using Laser Ablation-Inductively Coupled Plasma-Mass Spectrometry (La-Icp-Ms) and Its Potential Use in Geographic Sourcing. Talanta, 2018.

40. Aramendía, M., Rello, L., Vanhaecke, F., Resano, M., Direct Trace-Elemental Analysis of Urine Samples by Laser Ablation-Inductively Coupled Plasma Mass Spectrometry after Sample Deposition on Clinical Filter Papers. Analytical Chemistry, 2012. 84: p. 8682-8690.

41. Nischkauer, W., Vanhaecke, F., Bernacchi, S., Herwig, C., Limbeck, A., Radial Line-Scans as Representative Sampling Strategy in Dried-Droplet Laser Ablation of Liquid Samples Deposited on Pre-Cut Filter Paper Disks. Spectrochimica Acta Part B: Atomic Spectroscopy, 2014. 101: p. 123-129.

42. Latkoczy, C., Becker, S., Ducking, M., Gunther, D., Hoogewerff, J., Almirall, J., Buscaglia, J., Dobney, A., Koons, R., Montero, S., van der Peijl, G., Stoecklein, W., Trejos, T., Watling, J., Zdanowicz, V., Development and Evaluation of a Standard Method for the Quantitative Determination of Elements in Float Glass Samples by La-Icp-Ms. Journal of forensic sciences, 2005. 50: p. 1327-1341.

43. Trejos, T., Koons, R., Becker, S., Berman, T., Buscaglia, J., Duecking, M., Eckert-Lumsdon, T., Ernst, T., Hanlon, C., Heydon, A., Mooney, K., Nelson, R., Olsson, K., Palenik, C., Pollock, E., Rudell, D., Ryland, S., Tarifa, A., Valadez, M., Weis, P., Almirall, J., Cross-Validation and Evaluation of the Performance of Methods for the Elemental Analysis of Forensic Glass by M-Xrf, Icp-Ms, and LaIcp-Ms. Analytical and bioanalytical chemistry, 2013. 405: p. 5393-5409.

44. Trejos, T., Koons, R., Weis, P., Becker, S., Berman, T., Dalpe, C., Duecking, M., Buscaglia, J., Eckert-Lumsdon, T., Ernst, T., Hanlon, C., Heydon, A., Mooney, K., Nelson, R., Olsson, K., Schenk, E., Palenik, C., Pollock, E. C., Rudell, D., Ryland, S., Tarifa, A., Valadez, M., van Es, A., Zdanowicz, V., Almirall, J., Forensic Analysis of Glass by Micro-Xrf, Sn-Icp-Ms, La-Icp-Ms and La-Icp-Oes: Evaluation of the Performance of Different Criteria for Comparing Elemental Composition. Journal of Analytical Atomic Spectrometry, 2013. 28: p. 12701282. 
45. Weis, P., Ducking, M., Watzke, P., Menges, S., Becker, S., Establishing a Match Criterion in Forensic Comparison Analysis of Float Glass Using Laser Ablation Inductively Coupled Plasma Mass Spectrometry. Journal of Analytical Atomic Spectrometry, 2011. 26: p. 1273-1284.

46. Dorn, H., Ruddell, D., Heydon, A., Burton, B., Discrimination of Float Glass by La-Icp-Ms: Assessment of Exclusion Criteria Using Casework Samples. Canadian Society of Forensic Science Journal, 2015. 48: p. 85-96.

47. Berends-Montero, S., Wiarda, W., de Joode, P., van der Peijl, G., Forensic Analysis of Float Glass Using Laser Ablation Inductively Coupled Plasma Mass Spectrometry (La-Icp-Ms): Validation of a Method. Journal of Analytical Atomic Spectrometry, 2006. 21: p. 1185-1193.

48. Bommarito, C., A Likely Misguided Attempt at Changing the Way We Write Associative Reports That Could Have Far Reaching Impact on How Our Message Is Misunderstood, in 2009 Trace Evidence Symposium. 2009: Clearwater Beach, Florida.

49. The Composition and Manufacture of Glass and Its Domestic and Industrial Applications. Forensic Examination of Glass and Paint, ed. B. Caddy. 2001, London: Taylor \& Francis. p. 27-46.

50. Dorn, H., Ruddell, D., Heydon, A., Stefanescu, A., Stryjnik, A., Burton, B., Unusual Variation of Thickness within a Pane of Annealed Float Glass. Canadian Society of Forensic Science Journal, 2013. 46: p. 166-169.

51. Koons, R., Buscaglia, J., Bottrell, M., Miller, E., Forensic Glass Comparisons, in Forensic Science Handbook, R. Saferstein, Editor. 2002, Prentice Hall: Upper Saddle River, New Jersey. p. 183-184.

52. Standard Test Method for the Automated Determination of Refractive Index of Glass Samples Using the Oil Immersion Method and a Phase Contrast Microscope.

53. Ebdon, L., Evans, E., Fisher, A., Hill, S., An Introduction to Analytical Atomic Spectrometry. 1998: Wiley. 
54. de Hoffmann, E., Stroobant, V., Mass Spectrometry: Principles and Applications. 3rd ed. 2012: Wiley.

55. Latkoczy, C., Becker, S., Ducking, M., Gunther, D., Hoogewerff, J. A., Almirall, J. R., Buscaglia, J., Dobney, A., Koons, R. D., Montero, S., van der Peijl, G. J., Stoecklein, W. R., Trejos, T., Watling, J. R., Zdanowicz, V. S., Development and Evaluation of a Standard Method for the Quantitative Determination of Elements in Float Glass Samples by La-Icp-Ms. Journal of forensic sciences, 2005. 50: p. 1327-1341.

56. Campbell, G., Curran, J., The Interpretation of Elemental Composition Measurements from Forensic Glass Evidence Iii. Science \& Justice, 2009. 49: p. 2-7.

57. Curran, J., Triggs, C., Almirall, J., Buckleton, J., Walsh, K., The Interpretation of Elemental Composition Measurements from Forensic Glass Evidence: I. Science \& Justice, 1997. 37: p. 241-244.

58. Curran, J., Triggs, C., Almirall, J., Buckleton, J., Walsh, K., The Interpretation of Elemental Composition Measurements from Forensic Glass Evidence: Ii. Science \& Justice, 1997. 37: p. 245-249.

59. Koons, R., Buscaglia, J., Interpretation of Glass Composition Measurements: The Effects of Match Criteria on Discrimination Capability. Journal of forensic sciences, 2002. 47: p. 505-512.

60. ASTM E2927-16e1 Standard Test Method for Determination of Trace Elements in Soda-Lime Glass Samples Using Laser Ablation Inductively Coupled Plasma Mass Spectrometry for Forensic Comparisons. 2016, https://doi.org/10.1520/E2927-16E01, ASTM International: West Conshohocken, PA.

61. Limentani, G. B., Ringo Mc Fau - Ye, F., Ye F Fau - Berquist, M. L., Berquist Ml Fau - McSorley, E. O., McSorley, E. O., Beyond the T-Test: Statistical Equivalence Testing.

62. Corzo, R., Hoffman, T., Weis, P., Franco-Pedroso, J., Ramos, D., Almirall, J., The Use of La-Icp-Ms Databases to Calculate Likelihood Ratios for the Forensic Analysis of Glass Evidence. Talanta, 2018. 
63. ASTM Standard E1967-11a Standard Test Method for the Automated Determination of Refractive Index of Glass Samples Using the Oil Immersion Method and a Phase Contrast Microscope. 2011, https://doi.org/10.1520/E196711A, ASTM International: West Conshohocken, PA. 


\section{APPENDIX}

\section{M\&M Glass Samples}

\begin{tabular}{|c|c|c|c|c|}
\hline$\#$ & VIN & Year & Make & Model \\
\hline 1 & 4T1BE32K94U272646 & 2004 & Toyota & Camry \\
\hline 2 & 2HGFG3B54DH500424 & 2013 & Honda & Civic \\
\hline 3 & 4JGCB65E18A071448 & 2008 & Mercedes & R350 \\
\hline 4 & 2HGFG12629H527108 & 2009 & Honda & Civic \\
\hline 5 & 1FA6P8CF7G5224485 & 2016 & Ford & Mustang \\
\hline 6 & 1YVHP80C185M32614 & 2008 & Mazda & 6 \\
\hline 7 & WMWXP5C59G3B76438 & 2016 & Mini & Cooper \\
\hline 8 & JTDKTUD35CD500860 & 2012 & Toyota & Yaris \\
\hline 9 & JTDZN3EU1C3024669 & 2012 & Toyota & Prius v \\
\hline 10 & KNDMC5C16F6019328 & 2015 & $\mathrm{Kia}$ & Sedona \\
\hline 11 & 4T1BK1EB5DU003862 & 2013 & Toyota & Avalon \\
\hline 12 & 1HGCT1B38DA000184 & 2013 & Honda & Accord \\
\hline 13 & JM3KE4BE0D0100445 & 2013 & Mazda & $\mathrm{CX}-5$ \\
\hline 14 & 3N1CN7AP5CL809250 & 2012 & Nissan & Versa \\
\hline 15 & 3TMJU4GN0AM093246 & 2010 & Toyota & Tacoma \\
\hline 16 & WAUAFAFC6CN003676 & 2012 & Audi & A6 \\
\hline 17 & JTKJF5C70B3001720 & 2011 & Scion & $\mathrm{tC}$ \\
\hline 18 & ML32A3HJ6ЕH003209 & 2014 & Mitsubishi & Mirage \\
\hline 19 & WVWJK73C99P049019 & 2009 & Volkswagen & Passat \\
\hline 20 & 1HGCR2F37DA011773 & 2013 & Honda & Accord \\
\hline 21 & 2HGFG12607H511521 & 2007 & Honda & Civic \\
\hline 22 & 4JGCB65E59A094913 & 2009 & Mercedes & R-Class \\
\hline 23 & WBAVL1C56DVR91057 & 2013 & BMW & $\mathrm{X} 1$ \\
\hline 24 & ML32A3HJ4EH003547 & 2014 & Mitsubishi & Mirage \\
\hline 25 & KNAFW4A34A5148033 & 2010 & $\mathrm{Kia}$ & Forte \\
\hline 26 & WAUGNAF49HN009162 & 2017 & Audi & A4 \\
\hline 27 & KNADM4A34D6227438 & 2013 & $\mathrm{Kia}$ & Rio \\
\hline 28 & 2HGFB2F52CH300384 & 2012 & Honda & Civic \\
\hline 29 & 5TFEY5F18GX197447 & 2016 & Toyota & Tundra \\
\hline 30 & JM1BL1K53B1445800 & 2011 & Mazda & 3 \\
\hline 31 & 5NPDH4AEXBH019866 & 2011 & Hyundai & Elantra \\
\hline 32 & KNAFX4A65E5055079 & 2014 & $\mathrm{Kia}$ & Forte \\
\hline 33 & JF1ZNAA11D2703970 & 2013 & Scion & FR-S \\
\hline 34 & 2T1KU40E69C102910 & 2009 & Toyota & Matrix \\
\hline 35 & JTMBD33V065032593 & 2006 & Toyota & RAV4 \\
\hline 36 & 5TFRM5F14BX023073 & 2011 & Toyota & Tundra \\
\hline 37 & 5TDZA23C55S339489 & 2005 & Toyota & Sienna \\
\hline 38 & KNADM4A37D6178283 & 2013 & Kia & Rio \\
\hline 39 & JM1DKFB72G0129070 & 2016 & Mazda & CX-3 \\
\hline 40 & WA1EFCFS2GR000662 & 2016 & Audi & Q3 \\
\hline 41 & KNAFU4A29A5143195 & 2010 & Kia & Forte \\
\hline 42 & WBA1F5C58EVV98871 & 2014 & BMW & 2 Series \\
\hline 43 & 3MYDLBZV8GY100139 & 2016 & Scion & iA \\
\hline 44 & 1N4AA6AP7GC380842 & 2016 & Nissan & Maxima \\
\hline 45 & 4T1BK36B96U135066 & 2006 & Toyota & Avalon \\
\hline 46 & WBA3A9C59CF270171 & 2012 & BMW & 3 Series \\
\hline 47 & 5FNYF4H41CB001552 & 2012 & Honda & Pilot \\
\hline 48 & 5N1AZ2MH3FN202392 & 2015 & Nissan & Murano \\
\hline 49 & JHMGE88209S009320 & 2009 & Honda & Fit \\
\hline 50 & KMHTC6AD8EU195530 & 2014 & Hyundai & Veloster \\
\hline 51 & JM1CW2BL7E0166862 & 2014 & Mazda & 5 \\
\hline
\end{tabular}




\begin{tabular}{|c|c|c|c|c|}
\hline$\#$ & VIN & Year & Make & Model \\
\hline 52 & 2HGFC4B51GH301966 & 2016 & Honda & Civic \\
\hline 53 & 5TFEY5F14GX199602 & 2016 & Toyota & Tundra \\
\hline 54 & JM1DKFC70G0136470 & 2016 & Mazda & CX-3 \\
\hline 55 & JM1DKFC79G0136435 & 2016 & Mazda & $\mathrm{CX}-3$ \\
\hline 56 & 1G1ZB5EB5A4129282 & 2010 & Chevrolet & Malibu \\
\hline 57 & 1VWAT7A37GC002279 & 2016 & Volkswagen & Passat \\
\hline 58 & JTDKARFUXG3001654 & 2016 & Toyota & Prius \\
\hline 59 & JA32U2FU0EU010058 & 2014 & Mitsubishi & Lancer \\
\hline 60 & 1G1ZB5EB0AF210940 & 2010 & Chevrolet & Malibu \\
\hline 61 & 3FA6P08D7HR103611 & 2017 & Ford & Fusion \\
\hline 62 & 2LMTJ8KRXGBL39605 & 2016 & Lincoln & MKX \\
\hline 63 & KMHD74LF5HU166048 & 2017 & Hyundai & Elantra \\
\hline 64 & 4A3AB36FX9E043222 & 2009 & Mitsubishi & Galant \\
\hline 65 & 4A3AB36F49E015450 & 2009 & Mitsubishi & Galant \\
\hline 66 & 4A3AB36F39E029145 & 2009 & Mitsubishi & Galant \\
\hline 67 & 2C3CCAAGXFH842181 & 2015 & Chrysler & 300 \\
\hline 68 & 2HGFG3B59DH501522 & 2013 & Honda & Civic \\
\hline 69 & 2HGFB2F50DH504344 & 2013 & Honda & Civic \\
\hline 70 & JHMZF1D43BS008964 & 2011 & Honda & CR-Z \\
\hline 71 & 5J6RM4H35CL072875 & 2012 & Honda & CR-Z \\
\hline 72 & JHMZF1D48BS007552 & 2011 & Honda & CR-Z \\
\hline 73 & 2HKRM4H38FH627963 & 2015 & Honda & CR-Z \\
\hline 74 & 2HGFC2F52GH504878 & 2016 & Honda & Civic \\
\hline 75 & 5J6RM4H34FL000599 & 2015 & Honda & CR-Z \\
\hline 76 & 3CZRU6H39GM717785 & 2016 & Honda & HR-V \\
\hline 77 & 3HGGK5H58FM712771 & 2015 & Honda & Fit \\
\hline 78 & 2HGFG21506H707035 & 2006 & Honda & Civic \\
\hline 79 & 1FA6P8CF3G5236374 & 2016 & Ford & Mustang \\
\hline 80 & 1FTEX1CF1GFA20367 & 2016 & Ford & F-150 \\
\hline 81 & 1FTEX1CF7FFB30998 & 2015 & Ford & F-150 \\
\hline 82 & 2FMTK4J85FBB64687 & 2015 & Ford & Edge \\
\hline 83 & KL4CJFSB7FB044306 & 2015 & Buick & Encore \\
\hline 84 & 1LNHL9DK9EG608524 & 2014 & Lincoln & MKS \\
\hline 85 & 1G1RE6E47EU140143 & 2014 & Chevrolet & Volt \\
\hline 86 & 1GCRCREC3GZ171577 & 2016 & Chevrolet & Silverado 1500 \\
\hline 87 & 1G1ZC5E0XAF215588 & 2010 & Chevrolet & Malibu \\
\hline 88 & YS3FD79Y876001896 & 2007 & Saab & $9-3$ \\
\hline 89 & 1G1JC5SH2F4177056 & 2015 & Chevrolet & Sonic \\
\hline 90 & KNDMC5C14F6018761 & 2015 & Kia & Sedona \\
\hline 91 & 5XYPHDA55GG004235 & 2016 & Kia & Sorento \\
\hline 92 & KNDJN2A23F7115161 & 2015 & $\mathrm{Kia}$ & Soul \\
\hline 93 & KNAFK4A61F5256185 & 2015 & Kia & Forte \\
\hline 94 & KNDJT2A69C7365668 & 2012 & Kia & Soul \\
\hline 95 & KNADN5A31C6064782 & 2012 & Kia & Rio \\
\hline 96 & KNADM4A35D6250775 & 2013 & Kia & Rio \\
\hline 97 & KNAFU4A23A5809774 & 2010 & Kia & Forte \\
\hline 98 & KNAFU4A20A5061193 & 2010 & Kia & Forte \\
\hline 99 & KNAFU4A24A5196726 & 2010 & $\mathrm{Kia}$ & Forte \\
\hline 100 & KNDUP131646544318 & 2004 & Kia & Sedona \\
\hline 101 & KNDJC735685790186 & 2008 & Kia & Sorento \\
\hline 102 & KNDMB233466028628 & 2006 & $\mathrm{Kia}$ & Sedona \\
\hline 103 & KNDJC733855460026 & 2005 & Kia & Sorento \\
\hline 104 & 1C3CCCAB4FN500832 & 2015 & Chrysler & 200 \\
\hline 105 & JA4AZ3A30EZ000940 & 2014 & Mitsubishi & Outlander \\
\hline 106 & ML32A3HJ1EH003425 & 2014 & Mitsubishi & Mirage \\
\hline 107 & 2B3КA43DX9H576961 & 2009 & Dodge & Charger \\
\hline
\end{tabular}




\begin{tabular}{|c|c|c|c|c|}
\hline \# & VIN & Year & Make & Model \\
\hline 108 & 1C4RDJAG4FC709700 & 2015 & Dodge & Durango \\
\hline 109 & JA3AU26U88U042188 & 2008 & Mitsubishi & Lancer \\
\hline 110 & 4A3AB36F39E024088 & 2009 & Mitsubishi & Galant \\
\hline 111 & 4A3AB36F29E039116 & 2009 & Mitsubishi & Galant \\
\hline 112 & JA4AR4AU9CZ000785 & 2012 & Mitsubishi & Outlander \\
\hline 113 & JM1BM1L7XE1140067 & 2014 & Mazda & 3 \\
\hline 114 & JM1DE1KY7D0166100 & 2014 & Mazda & 2 \\
\hline 115 & JM1CW2BL1E0169143 & 2015 & Mazda & 5 \\
\hline 116 & 1FADP5AU6EL509856 & 2014 & Ford & C-Max Hybrid \\
\hline 117 & JM1DE1HYXB0105280 & 2011 & Mazda & 2 \\
\hline 118 & JM1BK323761529766 & 2006 & Mazda & 3 \\
\hline 119 & JM1GJ1V51E1101202 & 2014 & Mazda & 6 \\
\hline 120 & 1YVHZ8DH8C5M40525 & 2012 & Mazda & 6 \\
\hline 121 & 1N4AL2AP0AN450308 & 2010 & Nissan & Altima \\
\hline 122 & KMHDU4AD8AU171938 & 2010 & Hyundai & Elantra \\
\hline 123 & JS2YC415585103206 & 2008 & Suzuki & SX4 \\
\hline 124 & JS2RE9A32A6101695 & 2010 & Suzuki & Kizashi \\
\hline 125 & JS2RE9A36C6101041 & 2012 & Suzuki & Kizashi \\
\hline 126 & 1N4AL3AP5DC153542 & 2013 & Nissan & Altima \\
\hline 127 & 1N4AL11D46N373837 & 2006 & Nissan & Altima \\
\hline 128 & 1N4AL3AP0GC123773 & 2016 & Nissan & Altima \\
\hline 129 & 1N4AA6AP3GC378439 & 2016 & Nissan & Maxima \\
\hline 130 & 3N1BC13E57L352925 & 2007 & Nissan & Versa \\
\hline 131 & JN8AF5MR1BT006565 & 2011 & Nissan & Juke \\
\hline 132 & JN8AE2KP1E9107979 & 2014 & Nissan & Quest \\
\hline 133 & 5N1AZ2MH8FN203215 & 2015 & Nissan & Murano \\
\hline 134 & 5N1AR2MM0FC604332 & 2015 & Nissan & Pathfinder \\
\hline 135 & 3N1AB61E78L637820 & 2008 & Nissan & Sentra \\
\hline 136 & 3N1CN7AP8EL809326 & 2014 & Nissan & Versa \\
\hline 137 & JN8AF5MR6ET354981 & 2014 & Nissan & Juke \\
\hline 138 & 3N1AB7AP6FY215175 & 2015 & Nissan & Sentra \\
\hline 139 & 1N4AZ0CP2EC330348 & 2014 & Nissan & Leaf \\
\hline 140 & 3N1AB61EX8L637586 & 2008 & Nissan & Sentra \\
\hline 141 & JN8AZ28R59T100782 & 2009 & Nissan & Cube \\
\hline 142 & 1N4AL21E49C197032 & 2009 & Nissan & Altima \\
\hline 143 & WAUAFAFL0CA118555 & 2012 & Audi & A4 \\
\hline 144 & WVWLK73C87E003165 & 2007 & Volkswagen & Passat \\
\hline 145 & KM8SMDHF5FU099649 & 2015 & Hyundai & Santa Fe \\
\hline 146 & KM8JUCAC9AU071268 & 2010 & Hyundai & Tucson \\
\hline 147 & 5NPEU46F36H004326 & 2006 & Hyundai & Sonata \\
\hline 148 & 5NPE24AA4GH265905 & 2016 & Hyundai & Sonata \\
\hline 149 & KMHTC6AD9EU195701 & 2014 & Hyundai & Veloster \\
\hline 150 & KM8J3CA44GU039705 & 2016 & Hyundai & Tucson \\
\hline 151 & KMHCT4AE5DU423849 & 2013 & Hyundai & Accent \\
\hline 152 & KMHGN4JE1FU012205 & 2015 & Hyundai & Genesis \\
\hline 153 & KMHGC46E59U043461 & 2009 & Hyundai & Genesis \\
\hline 154 & 5NPE24AA7GH327619 & 2016 & Hyundai & Sonata \\
\hline 155 & KM8JN72D26U380194 & 2006 & Hyundai & Tucson \\
\hline 156 & 5NPE24AF0FH005401 & 2015 & Hyundai & Sonata \\
\hline 157 & WAUFFAFC6GN005150 & 2016 & Audi & A6 \\
\hline 158 & 1VWAP7A32CC062775 & 2012 & Volkswagen & Passat \\
\hline 159 & WVGBV3AX7DW592597 & 2013 & Volkswagen & Tiguan \\
\hline 160 & 3VW2K7AJ4CM385273 & 2012 & Volkswagen & Jetta \\
\hline 161 & WVGBV75N99W000785 & 2009 & Volkswagen & Tiguan \\
\hline 162 & 3VWJP7AT2DM675477 & 2013 & Volkswagen & Beetle \\
\hline 163 & JN1DV6AP2CM811431 & 2012 & Infiniti & G \\
\hline 164 & 3FAHP06Z17R191545 & 2007 & Ford & Fusion \\
\hline
\end{tabular}




\begin{tabular}{|c|c|c|c|c|}
\hline$\#$ & VIN & Year & Make & Model \\
\hline 165 & JH4KB2F56AC000729 & 2010 & Acura & RL \\
\hline 166 & 19UUB2F33FA005996 & 2015 & Acura & TLX \\
\hline 167 & 19UUB2F75FA001855 & 2015 & Acura & TLX \\
\hline 168 & 5J8TB4H34GL002597 & 2016 & Acura & RDX \\
\hline 169 & 2LMTJ8LR7GBL47532 & 2016 & Lincoln & MKX \\
\hline 170 & 19UUA8F23CA021785 & 2012 & Acura & $\mathrm{TL}$ \\
\hline 171 & 19UUA8F20CA012719 & 2012 & Acura & $\mathrm{TL}$ \\
\hline 172 & JH4CU2F44CC004593 & 2012 & Acura & TSX \\
\hline 173 & JH4KC1F38EC002509 & 2014 & Acura & RLX \\
\hline 174 & YV1MK672X92146049 & 2009 & Volvo & $\mathrm{C} 30$ \\
\hline 175 & YV4982DZ2A2058523 & 2010 & Volvo & XC60 \\
\hline 176 & YV1MC68267J021595 & 2007 & Volvo & $\mathrm{C} 70$ \\
\hline 177 & YV1622FS3C2037006 & 2012 & Volvo & S60 \\
\hline 178 & YV4CZ592361284695 & 2006 & Volvo & XC90 \\
\hline 179 & YV1622FS1C2087628 & 2012 & Volvo & S60 \\
\hline 180 & YV1622FS0C2102782 & 2012 & Volvo & S60 \\
\hline 181 & YV4952CZ9E1684771 & 2014 & Volvo & XC90 \\
\hline 182 & YV1952AS0E1179656 & 2014 & Volvo & S80 \\
\hline 183 & WBA3A5C55CF259029 & 2012 & BMW & 3 Series \\
\hline 184 & WMWXM5C55ET936691 & 2014 & Mini & Cooper \\
\hline 185 & WBA1F5C59EVV98894 & 2014 & BMW & 2 Series \\
\hline 186 & WBA5B1C52ED484411 & 2014 & BMW & 5 Series \\
\hline 187 & WBAVL1C58DVR88063 & 2013 & BMW & $\mathrm{X} 1$ \\
\hline 188 & WMWZC3C52BWH97939 & 2011 & Mini & Cooper Countryman \\
\hline 189 & WDDGF4HB6DR283277 & 2013 & Mercedes & C Class \\
\hline 190 & WDDGF4HB4CR213372 & 2012 & Mercedes & C Class \\
\hline 191 & 5TFRY5F12FX187772 & 2015 & Toyota & Tundra \\
\hline 192 & JTEBU5JR5E5155284 & 2014 & Toyota & 4Runner \\
\hline 193 & JF1GD75647G505362 & 2007 & Subaru & Impreza \\
\hline 194 & JF2SH6AC4AH737008 & 2010 & Subaru & Forester \\
\hline 195 & JF1GD67656H504575 & 2006 & Subaru & Impreza \\
\hline 196 & 4S3BNAA61F3003502 & 2015 & Subaru & Legacy \\
\hline 197 & JF1GE61659H515022 & 2009 & Subaru & Impreza \\
\hline 198 & 4S4WX82C864430559 & 2009 & Subaru & B9 Tribeca \\
\hline 199 & JF2SJADC7EH400163 & 2014 & Subaru & Forester \\
\hline 200 & 4S3BNAA69F3003523 & 2015 & Subaru & Legacy \\
\hline 201 & JF1GE61658H503418 & 2008 & Subaru & Impreza \\
\hline 202 & JF2SJAAC0EH409856 & 2014 & Subaru & Forester \\
\hline 203 & JF2SH61699H704494 & 2009 & Subaru & Forester \\
\hline 204 & 4S3BMCA65C3032608 & 2012 & Subaru & Legacy \\
\hline 205 & JF1GH61699H816786 & 2009 & Subaru & Impreza \\
\hline 206 & JF1GPAA63EH203000 & 2014 & Subaru & Impreza \\
\hline 207 & 3C4PDDBG3FT598049 & 2015 & Dodge & Journey \\
\hline 208 & ZACCJBAT1FPC09734 & 2015 & Jeep & Renegade \\
\hline 209 & ZACCJBAT5FPC27590 & 2015 & Jeep & Renegade \\
\hline 210 & 2C3CDZBT9GH108611 & 2016 & Dodge & Challenger \\
\hline
\end{tabular}


2. M\&M Database Control Charts for FGS1
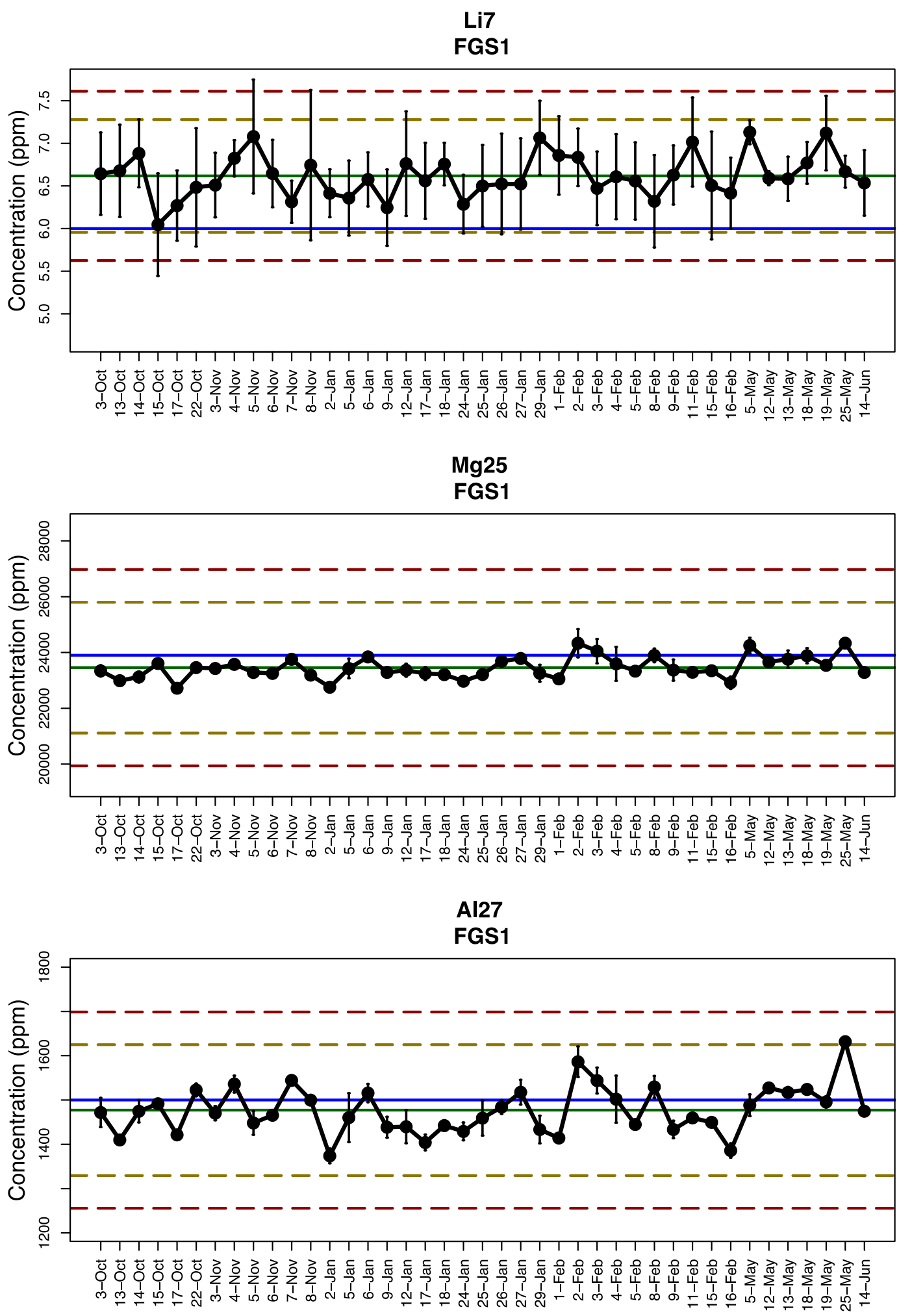

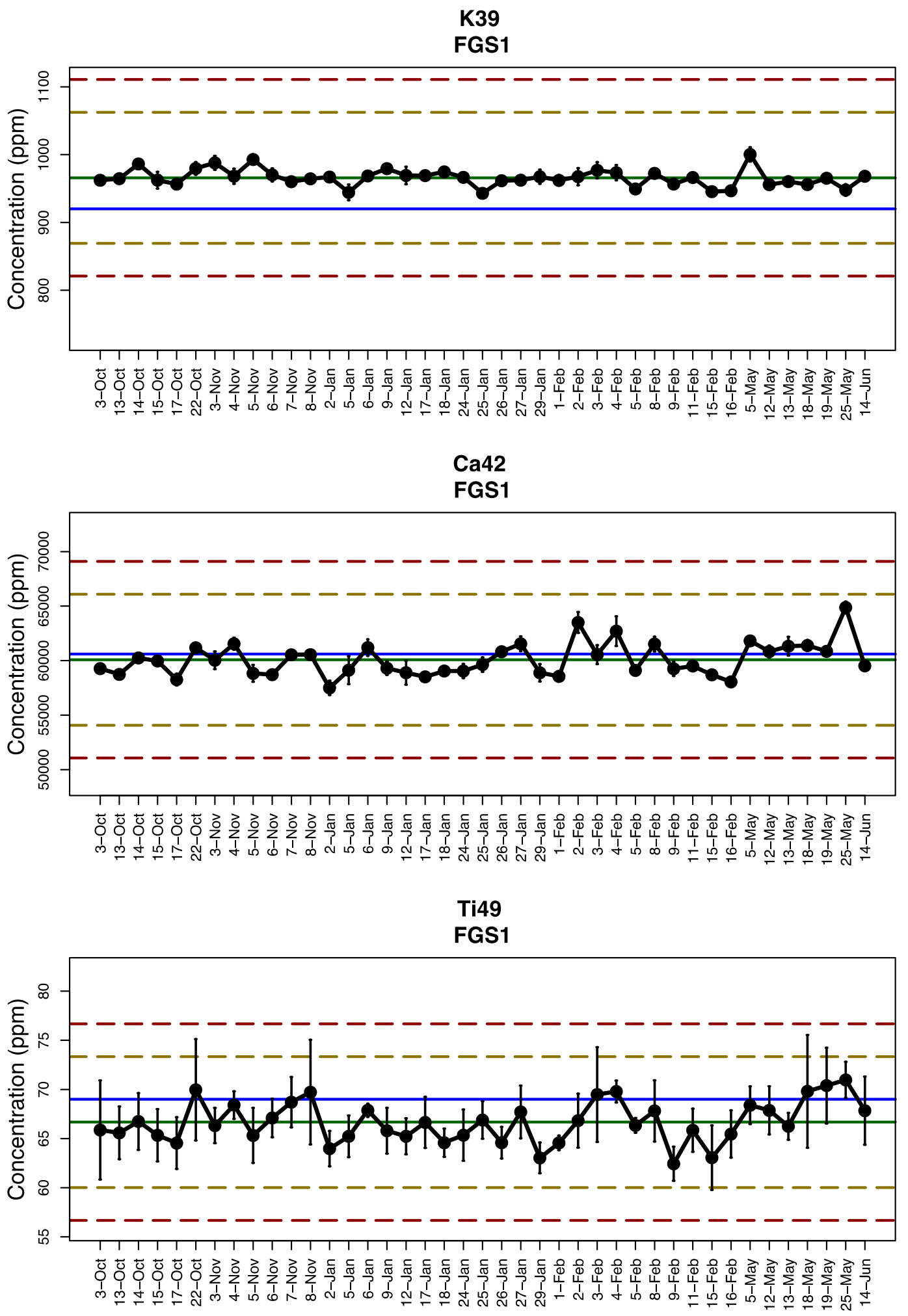

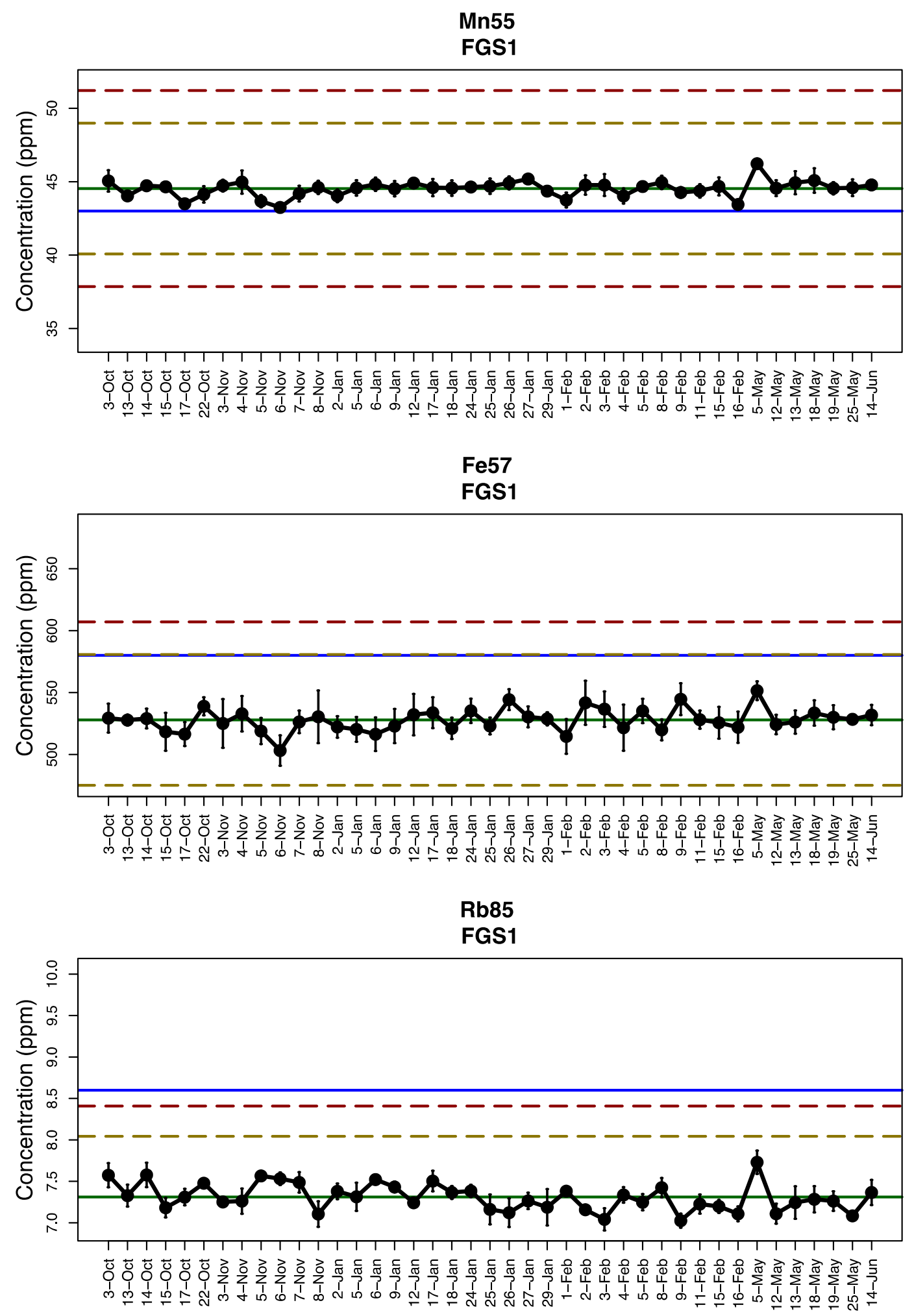

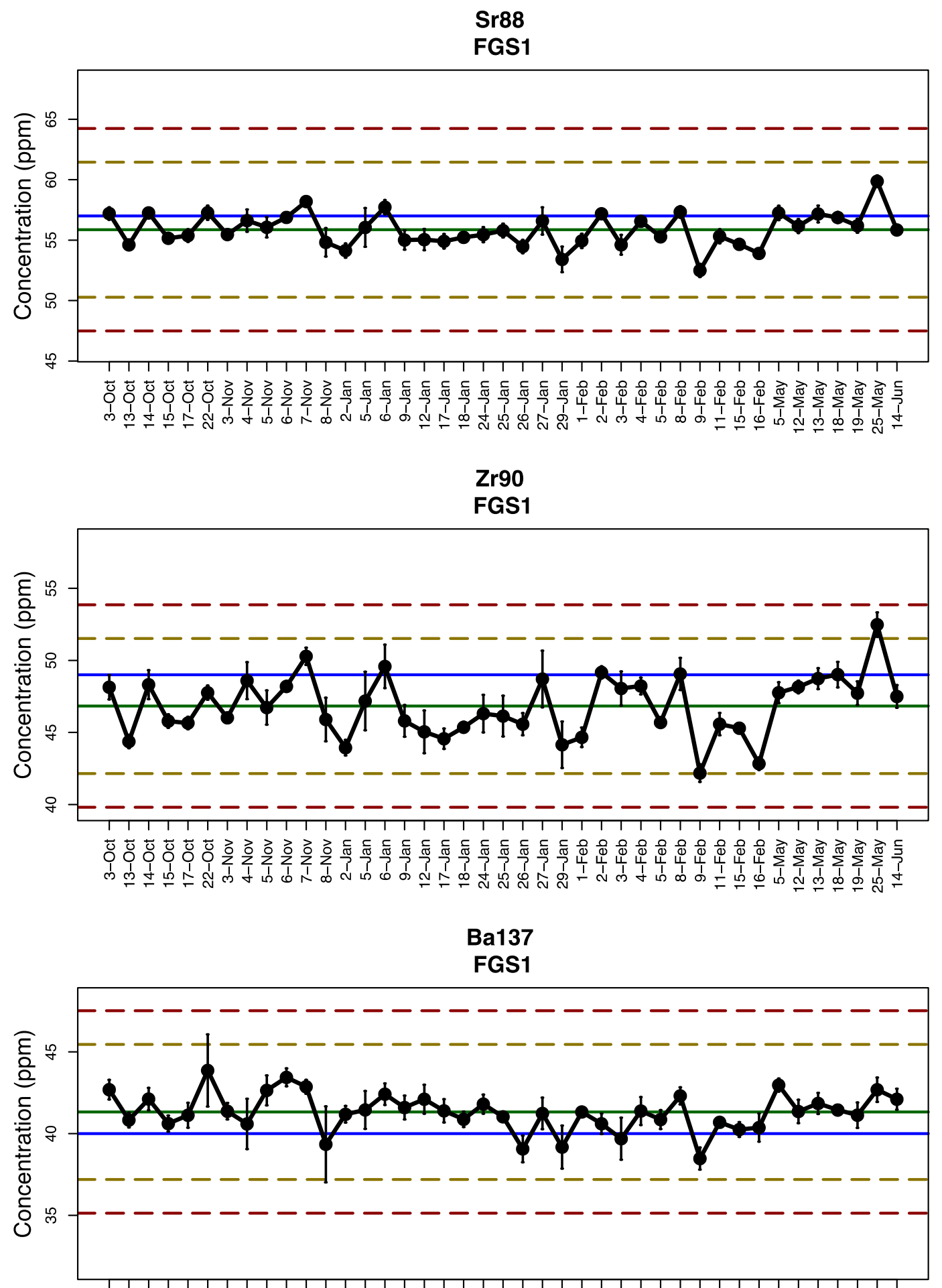

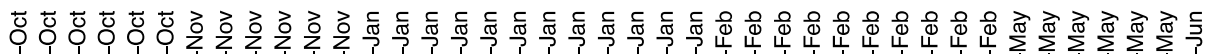

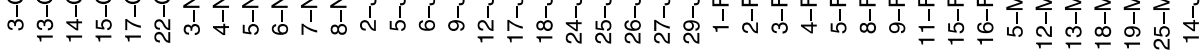



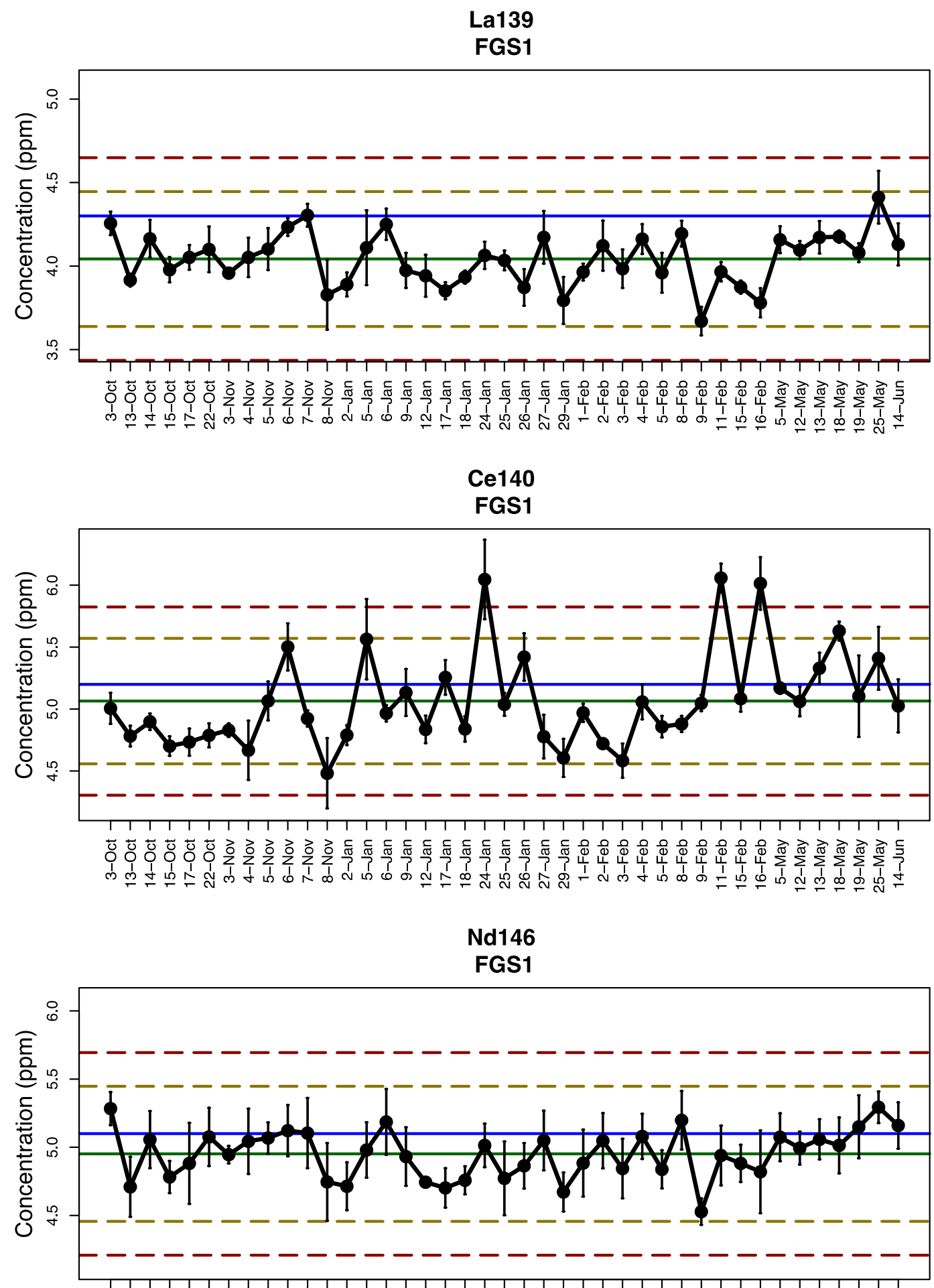

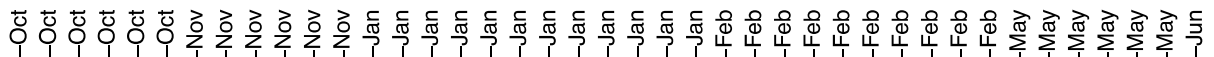

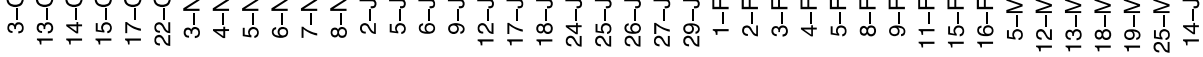



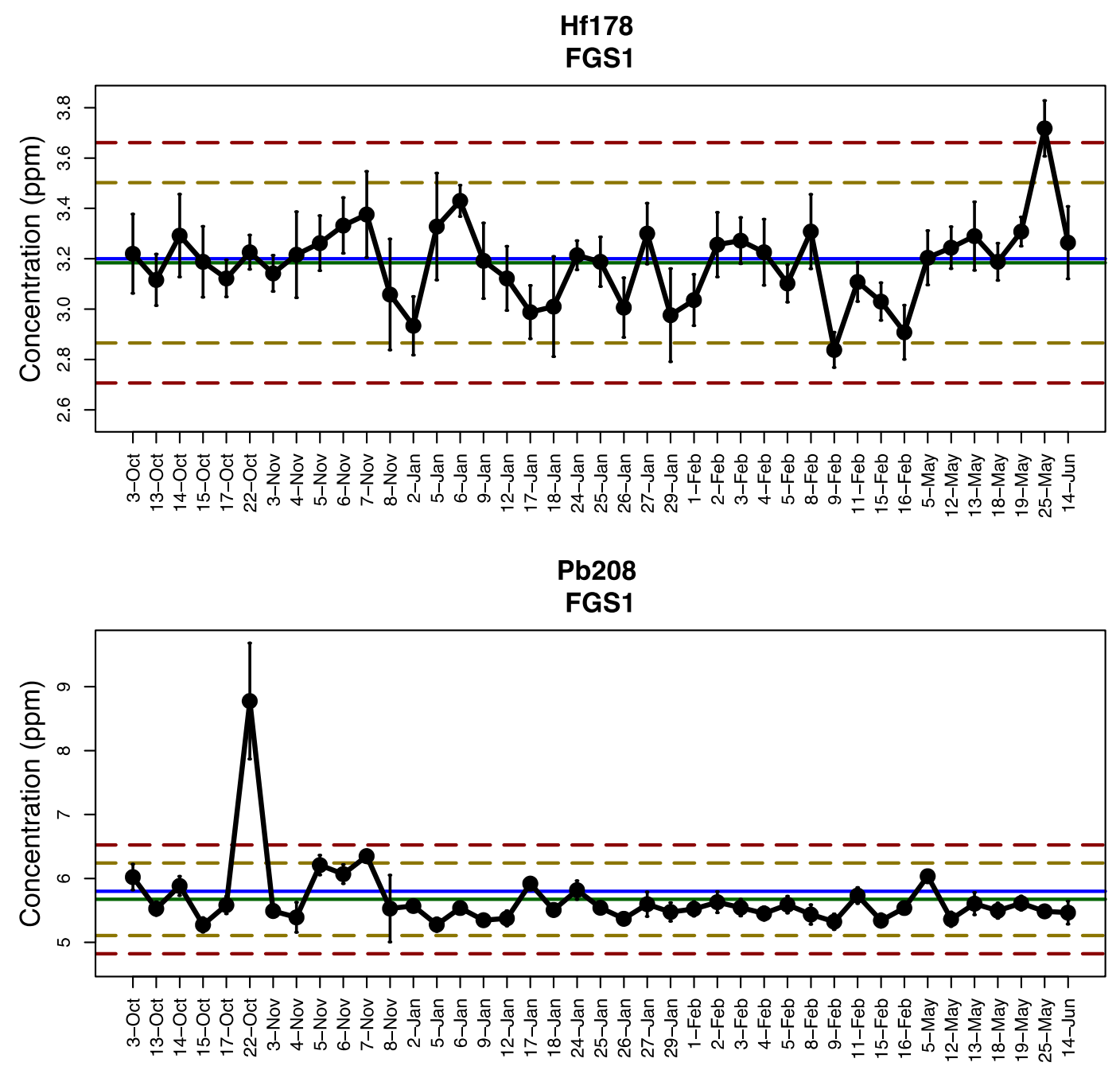
3. Thickness Measurements

\begin{tabular}{|c|c|c|c|}
\hline Sample & Thickness (mm) & Sample & Thickness (mm) \\
\hline 001.2 Inner & 2.07 & 020.1 Outer & 2.03 \\
\hline 001.2 Outer & 2.07 & 021.1 Inner & 2.11 \\
\hline 002.1 Inner & 1.90 & 021.1 Outer & 2.15 \\
\hline 002.1 Outer & 2.04 & 022.3 Inner & 2.20 \\
\hline 003.1 Inner & 2.20 & 022.3 Outer & 2.12 \\
\hline 003.1 Outer & 2.10 & 023.1 Inner & 1.57 \\
\hline 004.1 Inner & 2.03 & 023.1 Outer & 2.10 \\
\hline 004.1 Outer & 2.10 & 024.2 Inner & 2.09 \\
\hline 005.1 Inner & 1.94 & 024.2 Outer & 2.10 \\
\hline 005.1 Outer & 1.94 & 025.1 Inner & 2.11 \\
\hline 006.1 Inner & 2.10 & 025.1 Outer & 2.10 \\
\hline 006.1 Outer & 2.09 & 026.1 Inner & 1.63 \\
\hline 007.1 Inner & 1.76 & 026.1 Outer & 2.03 \\
\hline 007.1 Outer & 2.04 & 027.1 Inner & 1.80 \\
\hline 008.1 Inner & 1.84 & 027.1 Outer & 2.06 \\
\hline 008.1 Outer & 2.01 & 028.1 Inner & 1.85 \\
\hline 009.1 Inner & 1.80 & 028.1 Outer & 2.06 \\
\hline 009.1 Outer & 1.95 & 029.1 Inner & 2.26 \\
\hline 010.1 Inner & 2.06 & 029.1 Outer & 2.25 \\
\hline 010.1 Outer & 2.05 & 030.1 Inner & 2.03 \\
\hline 011.1 Inner & 1.85 & 030.1 Outer & 2.06 \\
\hline 011.1 Outer & 1.99 & 031.1 Inner & 2.07 \\
\hline 012.1 Inner & 2.04 & 031.1 Outer & 2.05 \\
\hline 012.1 Outer & 2.00 & 032.1 Inner & 2.10 \\
\hline 013.1 Inner & 1.93 & 032.1 Outer & 2.08 \\
\hline 013.1 Outer & 2.02 & 033.3 Inner & 2.00 \\
\hline 014.1 Inner & 2.11 & 033.3 Outer & 2.01 \\
\hline 014.1 Outer & 2.18 & 034.3 Inner & 2.01 \\
\hline 015.1 Inner & 2.25 & 034.3 Outer & 2.09 \\
\hline 015.1 Outer & 2.25 & 035.1 Inner & 2.09 \\
\hline 016.1 Inner & 1.60 & 035.1 Outer & 2.07 \\
\hline 016.1 Outer & 2.11 & 036.1 Inner & 2.19 \\
\hline 017.1 Inner & 2.04 & 036.1 Outer & 2.26 \\
\hline 017.1 Outer & 2.02 & 037.1 Inner & 2.00 \\
\hline 018.1 Inner & 2.06 & 037.1 Outer & 2.09 \\
\hline 018.1 Outer & 2.07 & 038.1 Inner & 1.84 \\
\hline 019.1 Inner & 1.64 & 038.1 Outer & 2.09 \\
\hline 019.1 Outer & 2.07 & 039.1 Inner & 1.82 \\
\hline 020.1 Inner & 2.02 & 039.1 Outer & 2.03 \\
\hline
\end{tabular}




\begin{tabular}{|c|c|c|c|}
\hline Sample & Thickness (mm) & Sample & Thickness (mm) \\
\hline 040.1 Inner & 1.63 & 061.1 Outer & 2.12 \\
\hline 040.1 Outer & 2.03 & 062.3 Inner & 2.22 \\
\hline 041.1 Inner & 2.09 & 062.3 Outer & 2.09 \\
\hline 041.1 Outer & 2.08 & 063.2 Inner & 2.09 \\
\hline 042.3 Inner & 1.63 & 063.2 Outer & 2.09 \\
\hline 042.3 Outer & 2.17 & 064.2 Inner & 2.00 \\
\hline 043.1 Inner & 1.59 & 064.2 Outer & 2.27 \\
\hline 043.1 Outer & 2.13 & 065.2 Inner & 2.01 \\
\hline 044.1 Inner & 2.05 & 065.2 Outer & 2.29 \\
\hline 044.1 Outer & 2.15 & 066.3 Inner & 2.05 \\
\hline 045.1 Inner & 2.09 & 066.3 Outer & 2.27 \\
\hline 045.1 Outer & 2.11 & 067.1 Inner & 2.27 \\
\hline 046.1 Inner & 1.63 & 067.1 Outer & 2.26 \\
\hline 046.1 Outer & 2.08 & 068.2 Inner & 1.89 \\
\hline 047.1 Inner & 1.87 & 068.2 Outer & 2.06 \\
\hline 047.1 Outer & 2.04 & 069.1 Inner & 2.07 \\
\hline 048.1 Inner & 2.02 & 069.1 Outer & 1.90 \\
\hline 048.1 Outer & 1.95 & 070.1 Inner & 2.03 \\
\hline 049.1 Inner & 1.84 & 070.1 Outer & 2.09 \\
\hline 049.1 Outer & 1.99 & 071.1 Inner & 2.05 \\
\hline 050.3 Inner & 2.10 & 071.1 Outer & 2.05 \\
\hline 050.3 Outer & 2.09 & 072.3 Inner & 2.04 \\
\hline 051.1 Inner & 1.96 & 072.3 Outer & 2.10 \\
\hline 051.1 Outer & 1.95 & 073.1 Inner & 2.00 \\
\hline 052.2 Inner & 1.85 & 073.1 Outer & 2.00 \\
\hline 052.2 Outer & 2.05 & 074.1 Inner & 1.80 \\
\hline 053.3 Inner & 2.25 & 074.1 Outer & 2.05 \\
\hline 053.3 Outer & 2.23 & 075.3 Inner & 2.10 \\
\hline 054.1 Inner & 2.04 & 075.3 Outer & 2.09 \\
\hline 054.1 Outer & 1.83 & 076.1 Inner & 1.63 \\
\hline 055.1 Inner & 1.85 & 076.1 Outer & 2.12 \\
\hline 055.1 Outer & 2.01 & 077.1 Inner & 1.88 \\
\hline 056.1 Inner & 2.25 & 077.1 Outer & 2.05 \\
\hline 056.1 Outer & 2.24 & 078.1 Inner & 2.10 \\
\hline 057.2 Inner & 2.11 & 078.1 Outer & 2.02 \\
\hline 057.2 Outer & 2.08 & 079.1 Inner & 1.96 \\
\hline 058.2 Inner & 1.98 & 079.1 Outer & 1.96 \\
\hline 058.2 Outer & 1.62 & 080.1 Inner & 2.18 \\
\hline 059.1 Inner & 1.95 & 080.1 Outer & 2.21 \\
\hline 059.1 Outer & 1.95 & 081.1 Inner & 2.22 \\
\hline 060.3 Inner & 2.26 & 081.1 Outer & 2.23 \\
\hline 060.3 Outer & 2.23 & 082.1 Inner & 2.11 \\
\hline 061.1 Inner & 2.11 & 082.1 Outer & 2.12 \\
\hline
\end{tabular}




\begin{tabular}{cc}
\hline Sample & Thickness $\mathbf{( m m})$ \\
\hline 083.1 Inner & 2.08 \\
083.1 Outer & 2.09 \\
084.1 Inner & 2.24 \\
084.1 Outer & 2.23 \\
085.1 Inner & 2.04 \\
085.1 Outer & 2.02 \\
086.1 Inner & 2.30 \\
086.1 Outer & 2.31 \\
087.1 Inner & 2.24 \\
087.1 Outer & 2.24 \\
088.1 Inner & 2.10 \\
088.1 Outer & 2.63 \\
089.1 Inner & 2.02 \\
089.1 Outer & 2.02 \\
090.1 Inner & 2.08 \\
090.1 Outer & 2.14 \\
091.1 Inner & 2.06 \\
091.1 Outer & 2.07 \\
092.1 Inner & 2.06 \\
092.1 Outer & 2.07 \\
093.1 Inner & 2.10 \\
093.1 Outer & 2.06 \\
094.1 Inner & 2.05 \\
094.1 Outer & 2.07 \\
095.1 Inner & 1.84 \\
095.1 Outer & 2.10 \\
096.1 Inner & 1.81 \\
096.1 Outer & 2.08 \\
097.1 Inner & 2.07 \\
097.1 Outer & 2.11 \\
098.1 Inner & 2.06 \\
098.1 Outer & 2.11 \\
099.1 Inner & 2.07 \\
099.1 Outer & 2.12 \\
100.1 Inner & 2.11 \\
100.1 Outer & 2.21 \\
101.1 Inner & 2.11 \\
101.1 Outer & 2.12 \\
102.1 Inner & 2.11 \\
102.1 Outer & 2.12 \\
103.1 Inner & 2.09 \\
103.1 Outer & 2.06 \\
104.1 Inner & 2.04 \\
\hline
\end{tabular}

\begin{tabular}{cc}
\hline Sample & Thickness $(\mathbf{m m})$ \\
\hline 104.1 Outer & 2.03 \\
105.1 Inner & 1.97 \\
105.1 Outer & 2.04 \\
106.1 Inner & 2.02 \\
106.1 Outer & 2.06 \\
107.1 Inner & 2.25 \\
107.1 Outer & 2.22 \\
108.1 Inner & 2.09 \\
108.1 Outer & 2.09 \\
109.1 Inner & 2.06 \\
109.1 Outer & 2.05 \\
110.1 Inner & 2.04 \\
110.1 Outer & 2.24 \\
111.1 Inner & 2.26 \\
111.1 Outer & 2.03 \\
112.1 Inner & 2.00 \\
112.1 Outer & 2.03 \\
113.1 Inner & 1.95 \\
113.1 Outer & 1.93 \\
114.1 Inner & 2.00 \\
114.1 Outer & 2.00 \\
115.1 Inner & 2.01 \\
115.1 Outer & 2.00 \\
116.1 Inner & 2.13 \\
116.1 Outer & 2.12 \\
117.1 Inner & 1.95 \\
117.1 Outer & 1.98 \\
118.1 Inner & 1.94 \\
118.1 Outer & 1.93 \\
119.1 Inner & 1.90 \\
119.1 Outer & 2.04 \\
120.1 Inner & 2.16 \\
120.1 Outer & 2.16 \\
121.1 Inner & 1.99 \\
121.1 Outer & 2.00 \\
122.1 Inner & 1.82 \\
122.1 Outer & 2.08 \\
123.1 Inner & 2.01 \\
123.1 Outer & 2.05 \\
124.1 Inner & 2.03 \\
124.1 Outer & 2.03 \\
125.1 Inner & 1.99 \\
125.1 Outer & 1.98 \\
\end{tabular}




\begin{tabular}{|c|c|c|c|}
\hline Sample & Thickness (mm) & Sample & Thickness (mm) \\
\hline 126.1 Inner & 1.99 & 147.1 Outer & 2.12 \\
\hline 126.1 Outer & 1.99 & 148.1 Inner & 2.05 \\
\hline 127.1 Inner & 2.09 & 148.1 Outer & 2.06 \\
\hline 127.1 Outer & 2.11 & 149.1 Inner & 2.11 \\
\hline 128.1 Inner & 2.02 & 149.1 Outer & 2.10 \\
\hline 128.1 Outer & 2.01 & 150.1 Inner & 1.63 \\
\hline 129.1 Inner & 2.06 & 150.1 Outer & 2.08 \\
\hline 129.1 Outer & 2.08 & 151.1 Inner & 2.07 \\
\hline 130.1 Inner & 2.13 & 151.1 Outer & 2.06 \\
\hline 130.1 Outer & 2.07 & 152.1 Inner & 1.81 \\
\hline 131.1 Inner & 1.93 & 152.1 Outer & 2.09 \\
\hline 131.1 Outer & 2.10 & 153.1 Inner & 1.84 \\
\hline 132.1 Inner & 1.95 & 153.1 Outer & 2.07 \\
\hline 132.1 Outer & 1.95 & 154.1 Inner & 2.04 \\
\hline 133.1 Inner & 1.98 & 154.1 Outer & 2.07 \\
\hline 133.1 Outer & 1.97 & 155.1 Inner & 2.10 \\
\hline 134.1 Inner & 2.04 & 155.1 Outer & 2.14 \\
\hline 134.1 Outer & 1.95 & 156.1 Inner & 2.06 \\
\hline 135.1 Inner & 2.03 & 156.1 Outer & 2.07 \\
\hline 135.1 Outer & 2.04 & 157.1 Inner & 1.63 \\
\hline 136.1 Inner & 2.12 & 157.1 Outer & 2.11 \\
\hline 136.1 Outer & 2.12 & 158.1 Inner & 2.06 \\
\hline 137.1 Inner & 1.99 & 158.1 Outer & 2.09 \\
\hline 137.1 Outer & 2.00 & 159.1 Inner & 1.64 \\
\hline 138.1 Inner & 2.13 & 159.1 Outer & 2.05 \\
\hline 138.1 Outer & 2.10 & 160.1 Inner & 1.61 \\
\hline 139.1 Inner & 2.00 & 160.1 Outer & 2.12 \\
\hline 139.1 Outer & 2.00 & 161.1 Inner & 1.68 \\
\hline 140.1 Inner & 2.02 & 161.1 Outer & 2.05 \\
\hline 140.1 Outer & 2.00 & 162.1 Inner & 1.60 \\
\hline 141.1 Inner & 1.75 & 162.1 Outer & 2.09 \\
\hline 141.1 Outer & 1.96 & 163.1 Inner & 1.96 \\
\hline 142.1 Inner & 2.14 & 163.1 Outer & 2.05 \\
\hline 142.1 Outer & 2.14 & 164.1 Inner & 2.01 \\
\hline 143.1 Inner & 1.61 & 164.1 Outer & 2.10 \\
\hline 143.1 Outer & 2.11 & 165.1 Inner & 2.09 \\
\hline 144.1 Inner & 2.01 & 165.1 Outer & 2.05 \\
\hline 144.1 Outer & 2.03 & 166.1 Inner & 1.84 \\
\hline 145.1 Inner & 1.67 & 166.1 Outer & 2.01 \\
\hline 145.1 Outer & 2.06 & 167.1 Inner & 1.84 \\
\hline 146.1 Inner & 2.05 & 167.1 Outer & 2.00 \\
\hline 146.1 Outer & 2.05 & 168.1 Inner & 2.04 \\
\hline 147.1 Inner & 2.11 & 168.1 Outer & 2.00 \\
\hline
\end{tabular}




\begin{tabular}{cc}
\hline Sample & Thickness $(\mathbf{m m})$ \\
\hline 169.1 Inner & 2.23 \\
169.1 Outer & 2.24 \\
170.1 Inner & 1.88 \\
170.1 Outer & 1.98 \\
171.1 Inner & 1.83 \\
171.1 Outer & 1.96 \\
172.1 Inner & 1.99 \\
172.1 Outer & 2.05 \\
173.1 Inner & 1.93 \\
173.1 Outer & 2.04 \\
174.1 Inner & 1.64 \\
174.1 Outer & 2.03 \\
175.2 Inner & 2.09 \\
175.2 Outer & 2.61 \\
176.1 Inner & 1.61 \\
176.1 Outer & 2.12 \\
177.1 Inner & 2.12 \\
177.1 Outer & 2.61 \\
178.1 Inner & 2.20 \\
178.1 Outer & 2.53 \\
179.1 Inner & 2.09 \\
179.1 Outer & 2.60 \\
180.1 Inner & 2.09 \\
180.1 Outer & 2.59 \\
181.1 Inner & 2.11 \\
181.1 Outer & 2.58 \\
182.1 Inner & 1.62 \\
182.1 Outer & 2.09 \\
183.1 Inner & 1.62 \\
183.1 Outer & 2.08 \\
184.1 Inner & 1.59 \\
184.1 Outer & 2.10 \\
185.1 Inner & 1.61 \\
185.1 Outer & 2.17 \\
186.1 Inner & 1.62 \\
186.1 Outer & 2.09 \\
187.1 Inner & 1.54 \\
187.1 Outer & 2.11 \\
188.1 Inner & 1.60 \\
188.1 Outer & 2.09 \\
189.1 Inner & 1.79 \\
189.1 Outer & 1.80 \\
190.1 Inner & 1.78 \\
\hline
\end{tabular}

\begin{tabular}{cc}
\hline Sample & Thickness $(\mathbf{m m})$ \\
\hline 190.1 Outer & 1.77 \\
191.1 Inner & 2.25 \\
191.1 Outer & 2.25 \\
192.1 Inner & 2.17 \\
192.1 Outer & 2.20 \\
193.1 Inner & 1.97 \\
193.1 Outer & 1.99 \\
194.1 Inner & 1.99 \\
194.1 Outer & 2.01 \\
195.1 Inner & 1.97 \\
195.1 Outer & 1.93 \\
196.1 Inner & 1.96 \\
196.1 Outer & 1.99 \\
197.1 Inner & 2.03 \\
197.1 Outer & 2.06 \\
198.1 Inner & 2.02 \\
198.1 Outer & 1.99 \\
199.1 Inner & 2.05 \\
199.1 Outer & 2.06 \\
200.1 Inner & 2.03 \\
200.1 Outer & 1.99 \\
201.1 Inner & 1.94 \\
201.1 Outer & 1.94 \\
202.1 Inner & 2.00 \\
202.1 Outer & 1.99 \\
203.1 Inner & 1.95 \\
203.1 Outer & 1.94 \\
204.1 Inner & 2.10 \\
204.1 Outer & 2.11 \\
205.1 Inner & 2.06 \\
205.1 Outer & 2.05 \\
206.1 Inner & 1.94 \\
206.1 Outer & 1.94 \\
207.2 Inner & 2.06 \\
207.2 Outer & 2.27 \\
208.1 Inner & 1.68 \\
208.1 Outer & 2.13 \\
209.1 Inner & 1.60 \\
209.1 Outer & 2.08 \\
210.1 Inner & 2.06 \\
210.1 Outer & 2.05 \\
\hline
\end{tabular}


4. Comparison of First Inter-laboratory K glasses to Duplicate in Database

- K1 Inner vs. Sample 110 Inner

Li7

K1 Inner vs Sample 110 Inner

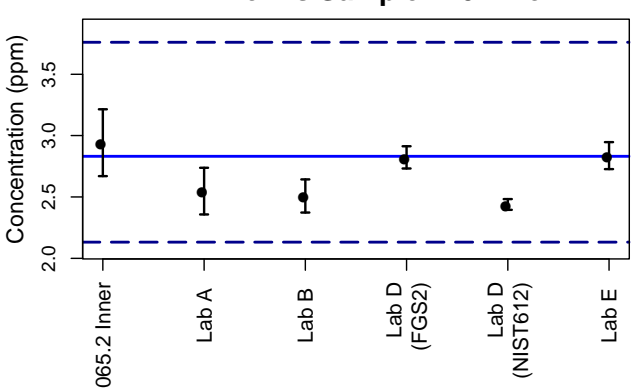

Al27

K1 Inner vs Sample 110 Inner

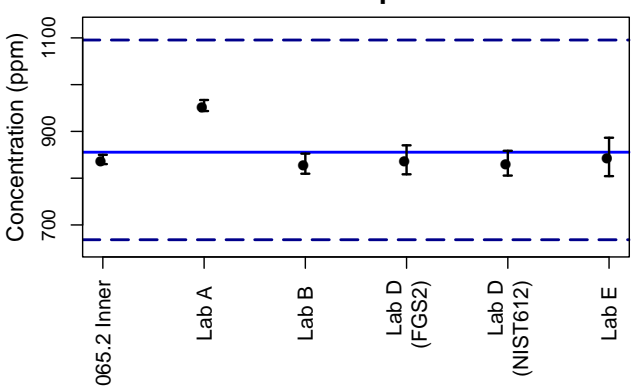

Ca42

K1 Inner vs Sample 110 Inner

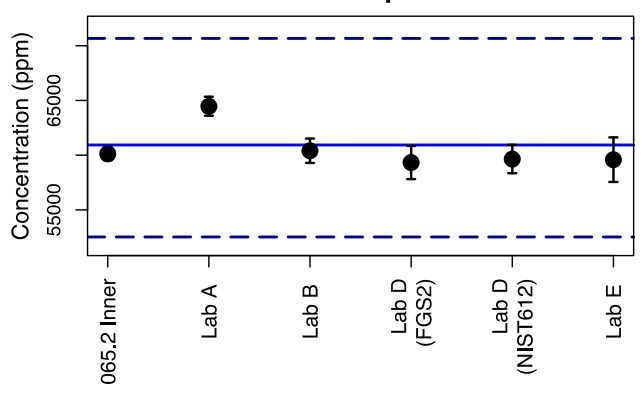

Mn55

K1 Inner vs Sample 110 Inner

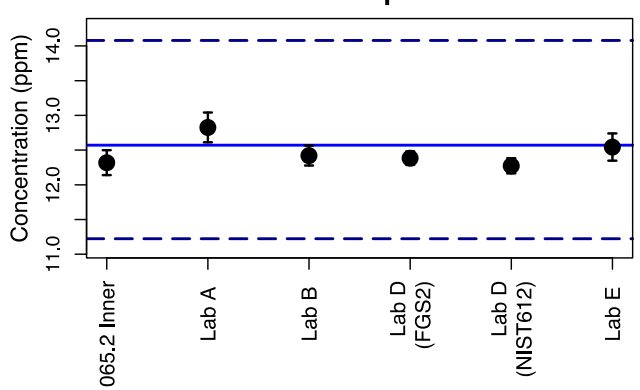

Mg25

K1 Inner vs Sample 110 Inner

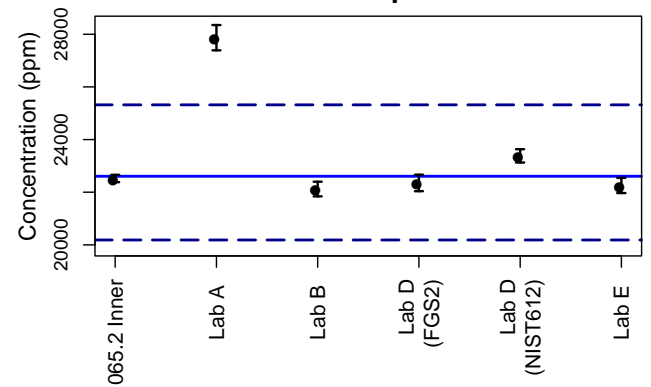

K39

K1 Inner vs Sample 110 Inner

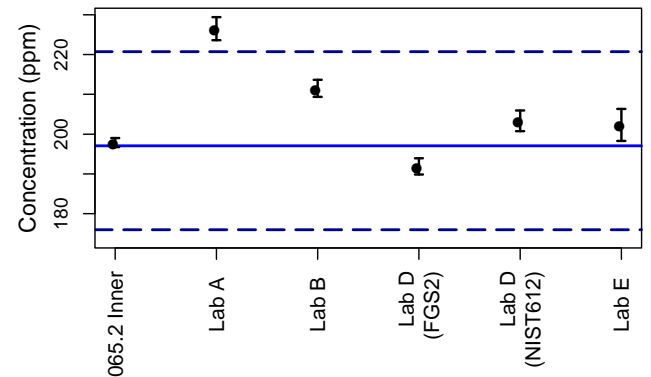

Ti49

K1 Inner vs Sample 110 Inner

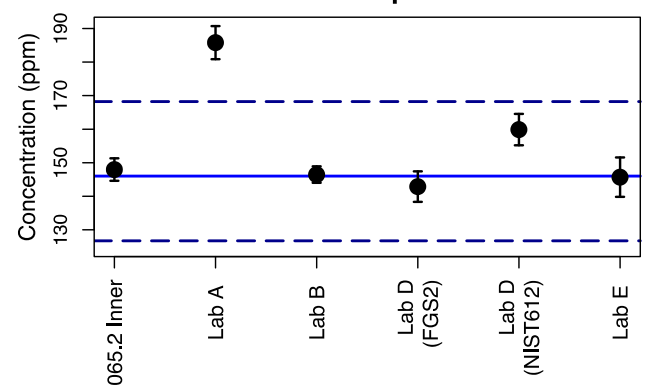

Fe57

K1 Inner vs Sample 110 Inner

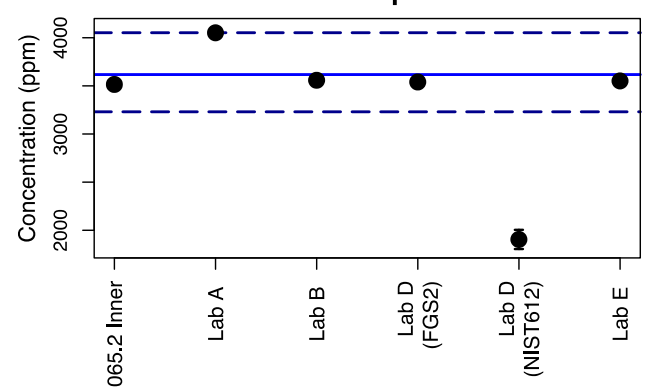


Rb85

K1 Inner vs Sample 110 Inner

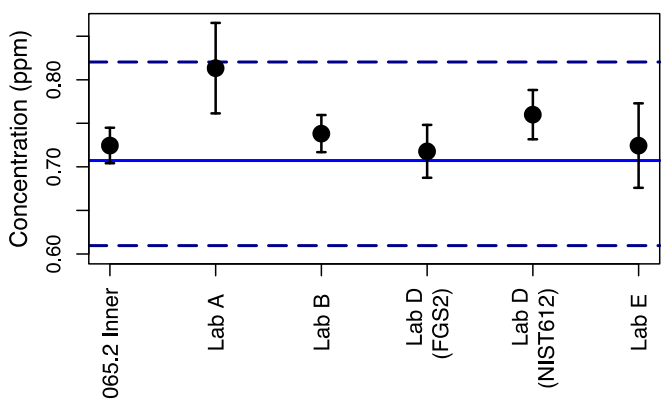

Zr90

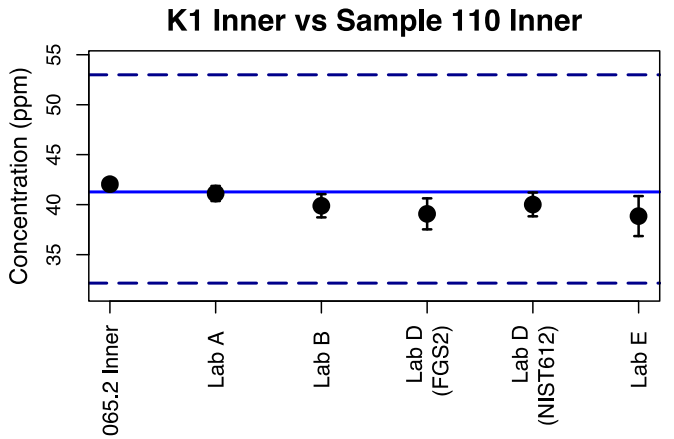

La139

K1 Inner vs Sample 110 Inner

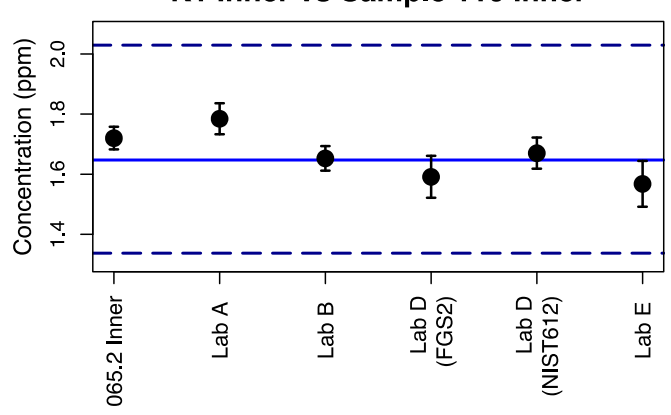

Nd146

K1 Inner vs Sample 110 Inner

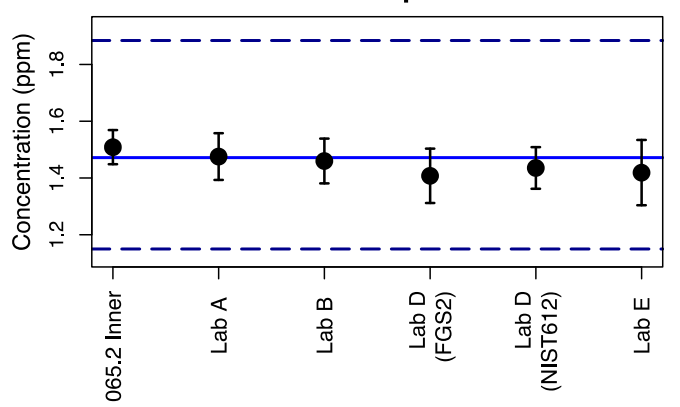

Sr88

K1 Inner vs Sample 110 Inner

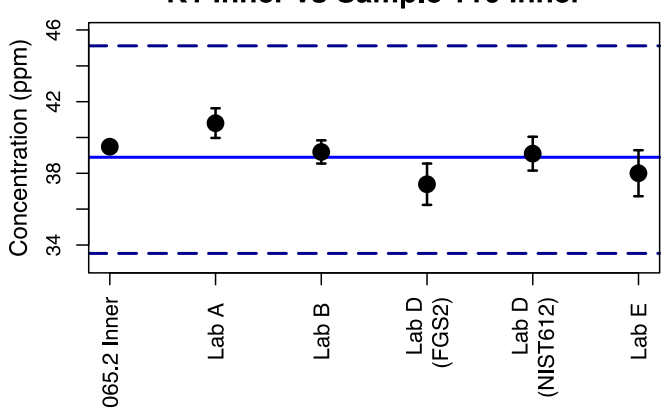

Ba137

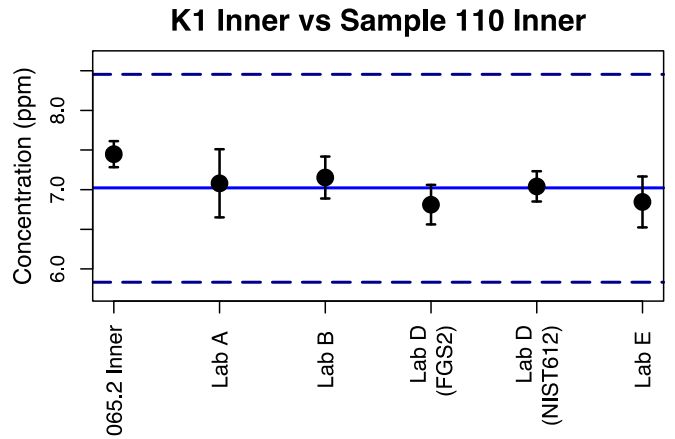

Ce140

K1 Inner vs Sample 110 Inner

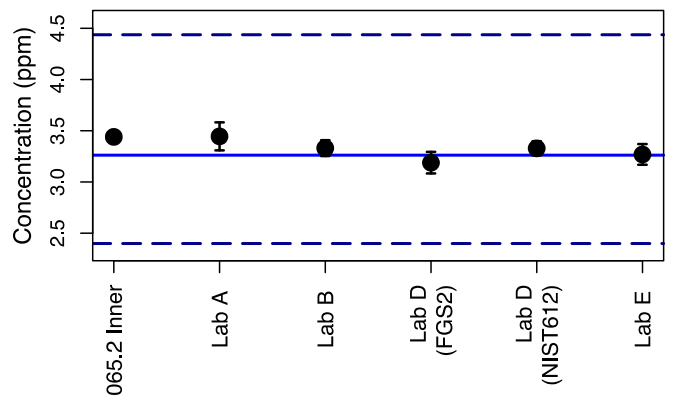

Pb208

K1 Inner vs Sample 110 Inner

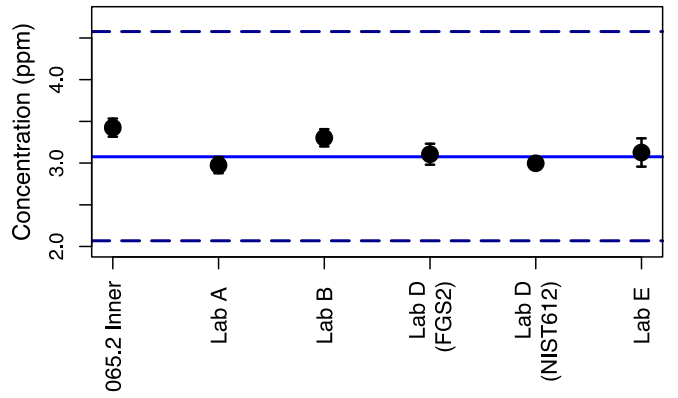


- K1 Outer vs. Sample 110 Outer

Li7

K1 Outer vs Sample 110 Outer

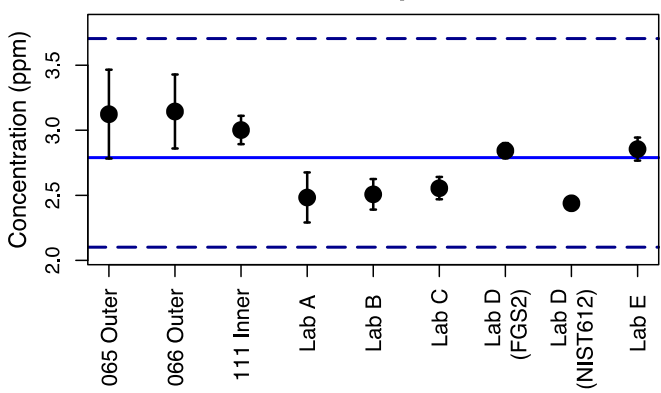

Al27

K1 Outer vs Sample 110 Outer

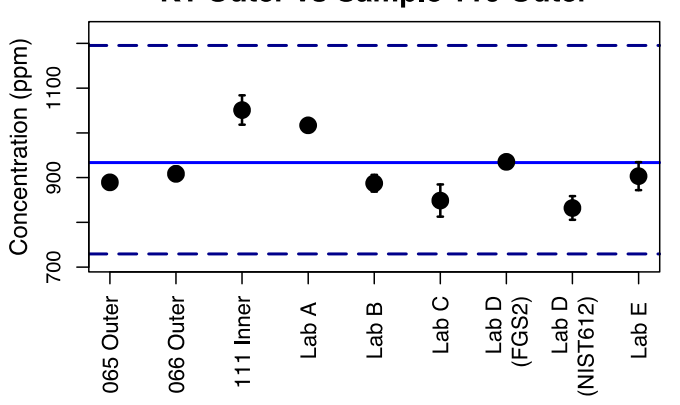

Ca42

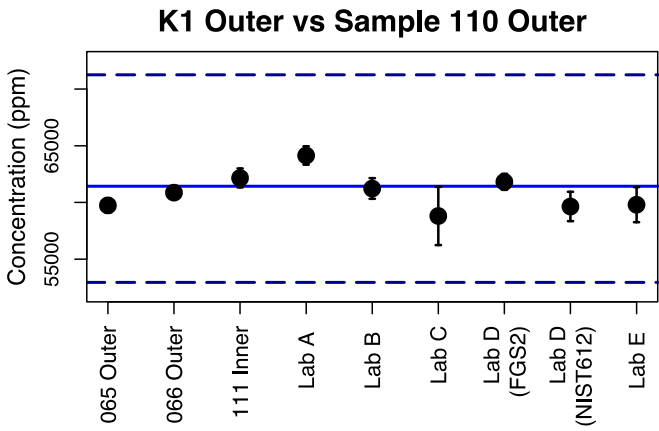

Mn55

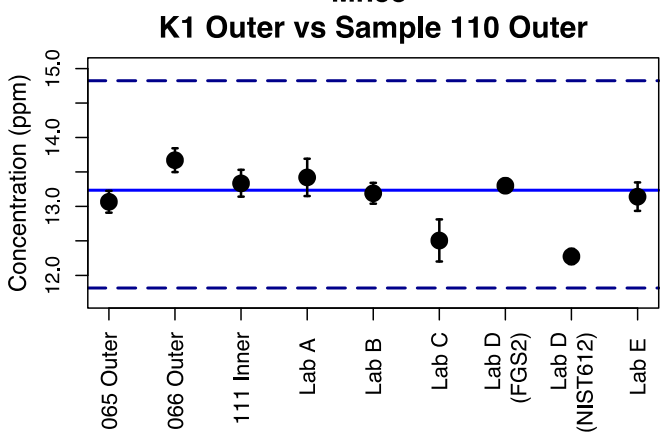

Mg25

K1 Outer vs Sample 110 Outer

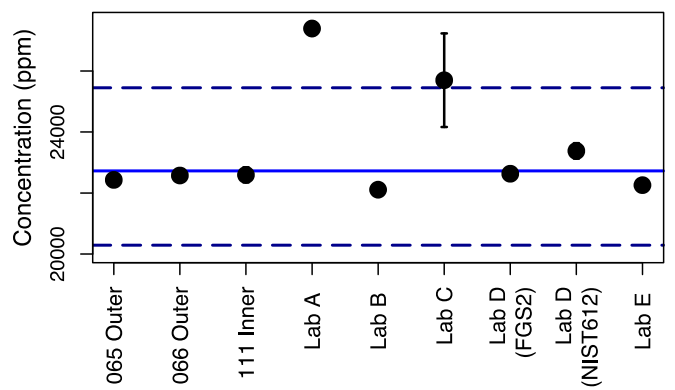

K39

K1 Outer vs Sample 110 Outer

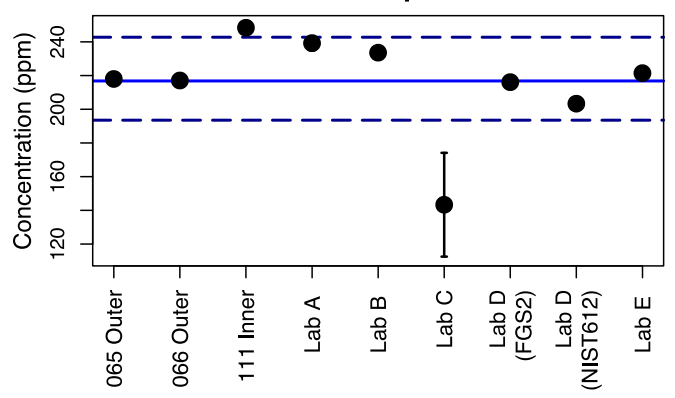

Ti49

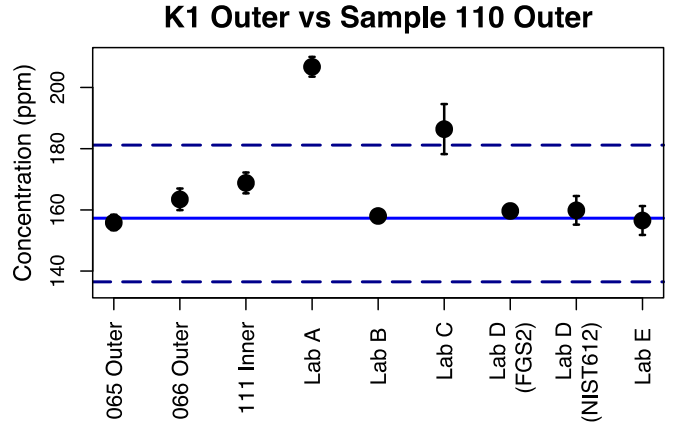

Fe57

K1 Outer vs Sample 110 Outer

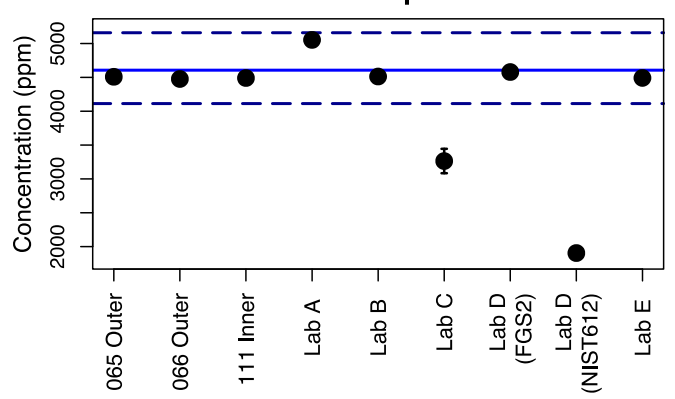


Rb85

K1 Outer vs Sample 110 Outer

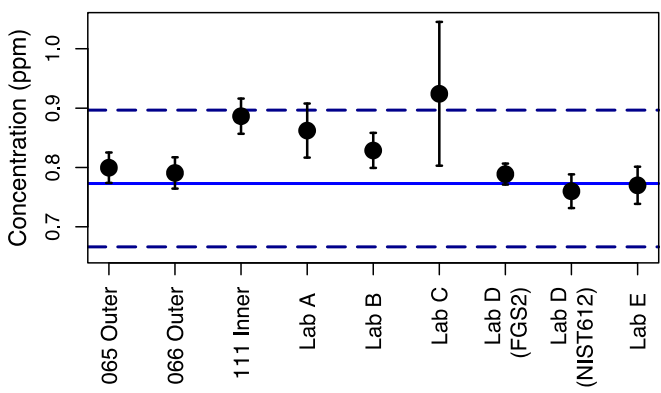

Zr90

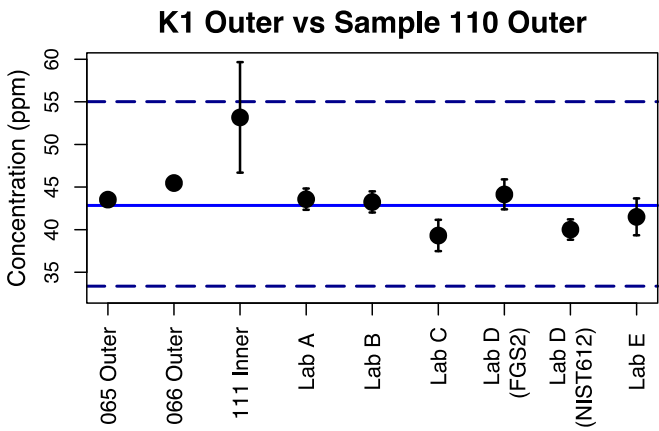

La139

K1 Outer vs Sample 110 Outer

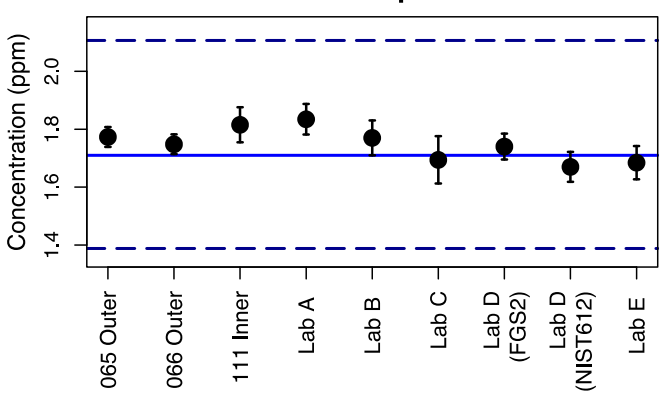

Nd146

K1 Outer vs Sample 110 Outer

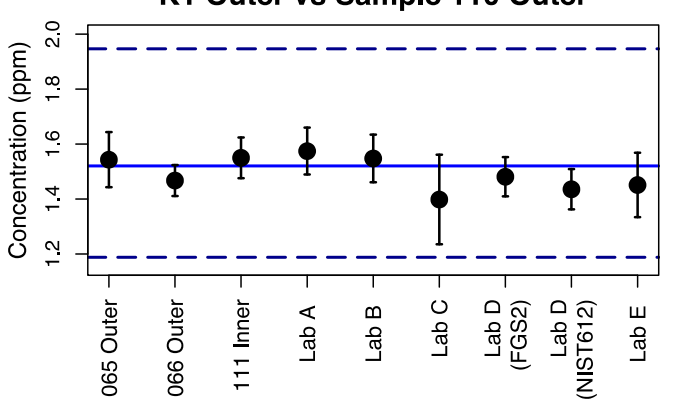

Sr88

K1 Outer vs Sample 110 Outer

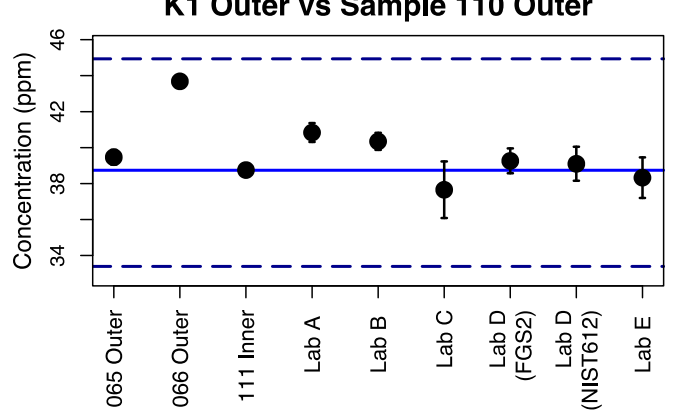

Ba137

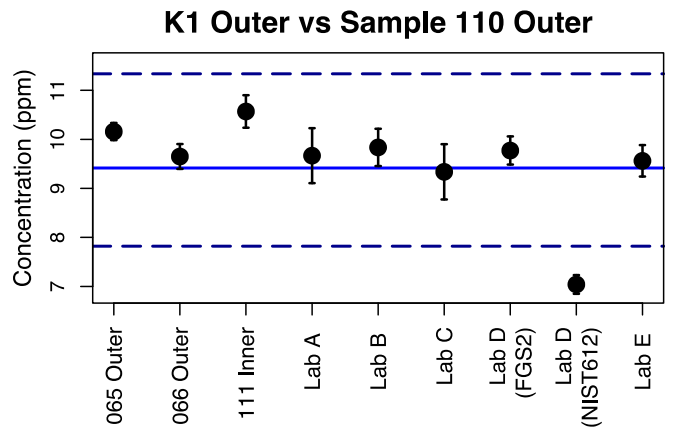

Ce140

K1 Outer vs Sample 110 Outer

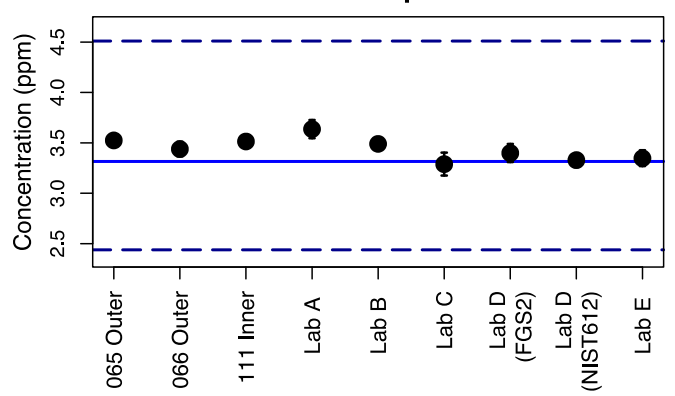

Pb208

K1 Outer vs Sample 110 Outer

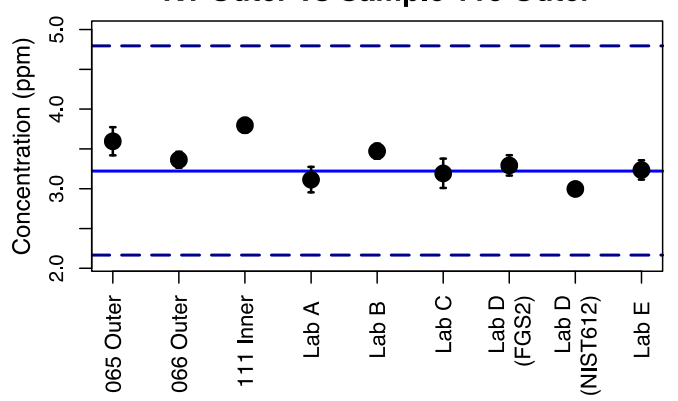


- $\quad$ K2 vs. Sample 201

Li7

K2 Inner vs Sample 201 Inner

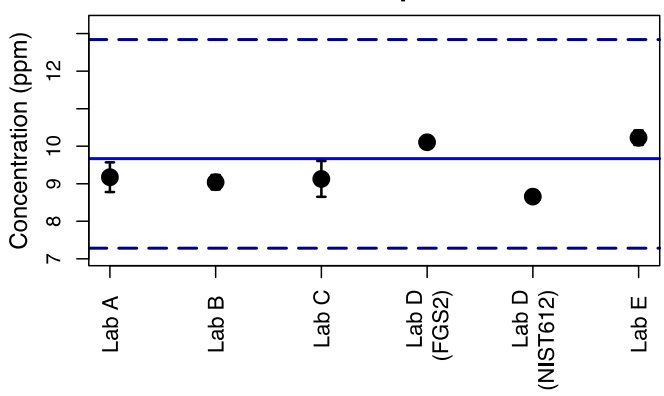

Al27

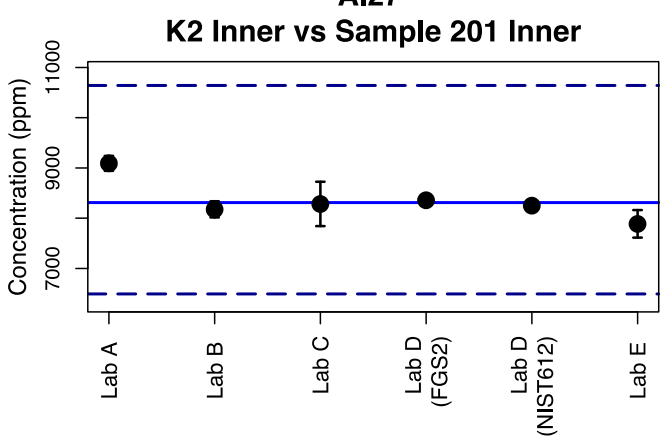

Ca42

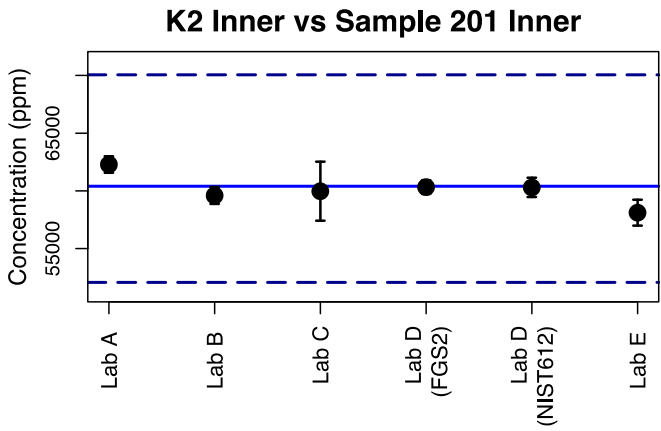

Mn55

K2 Inner vs Sample 201 Inner

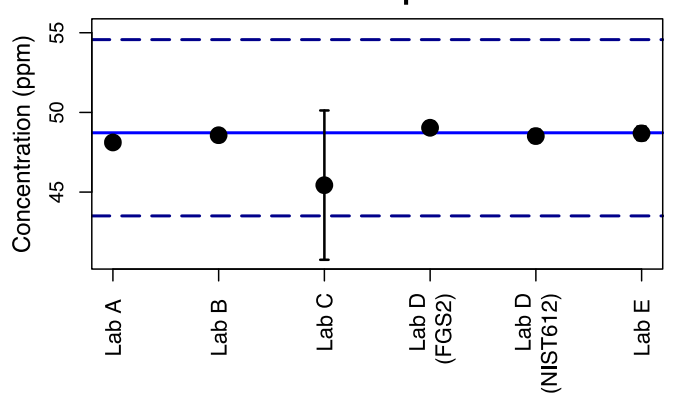

Mg25

K2 Inner vs Sample 201 Inner

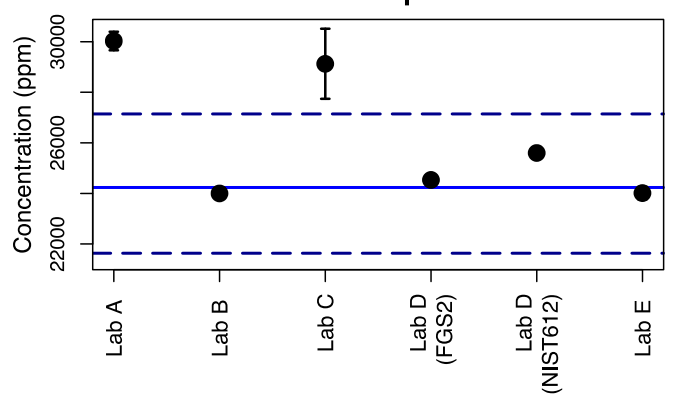

K39

K2 Inner vs Sample 201 Inner

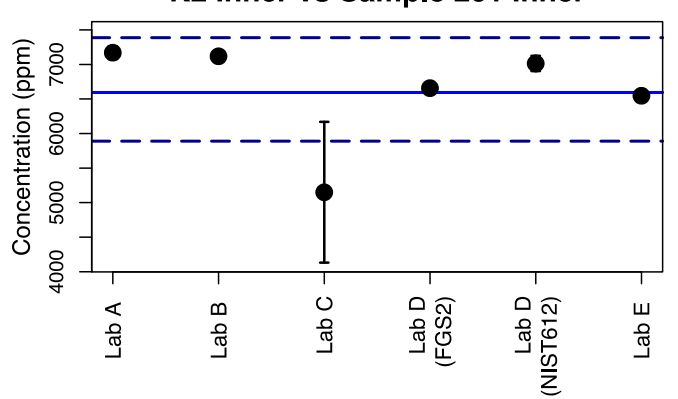

Ti49

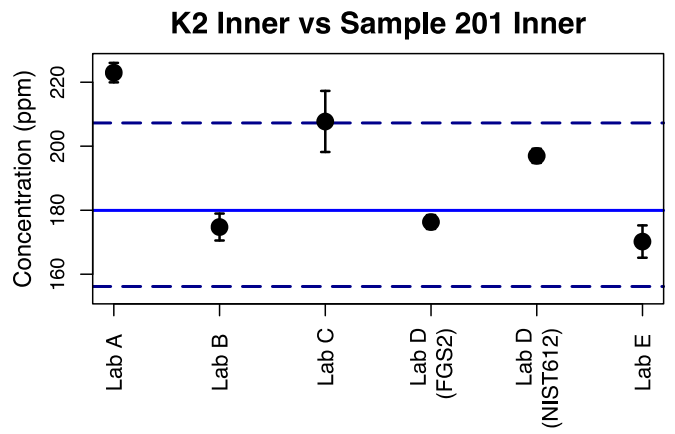

Fe57

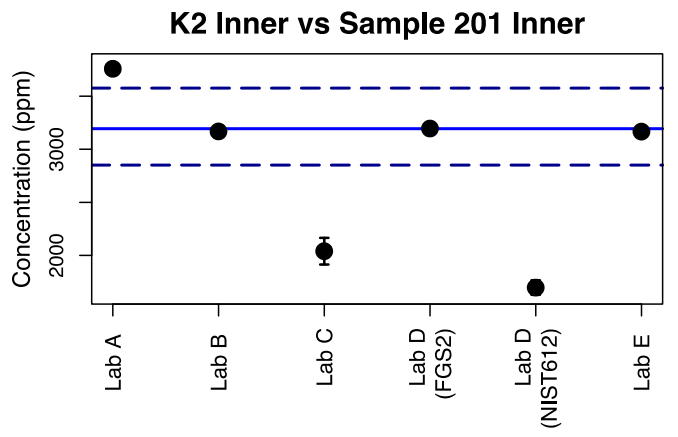


Rb85

K2 Inner vs Sample 201 Inner

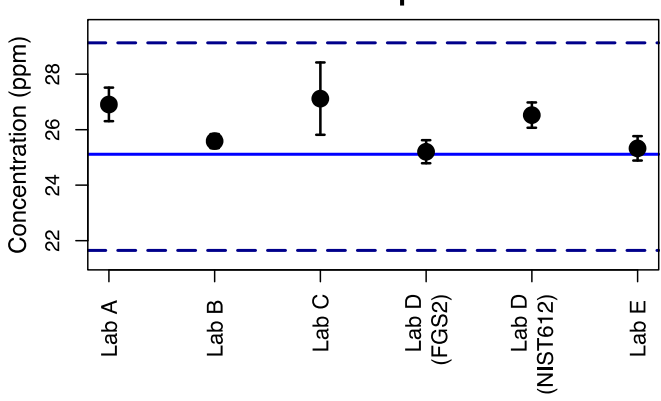

Zr90

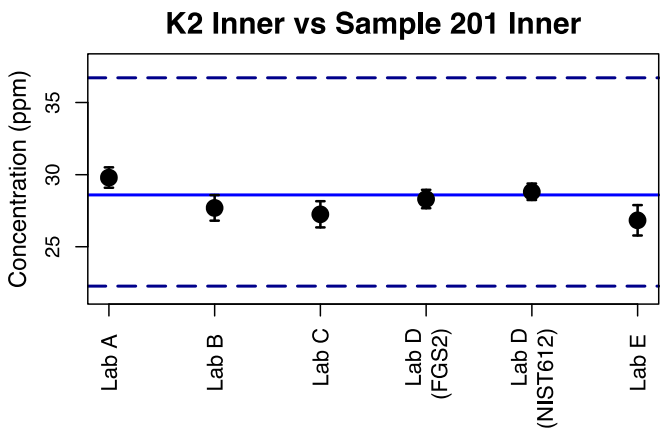

La139

K2 Inner vs Sample 201 Inner

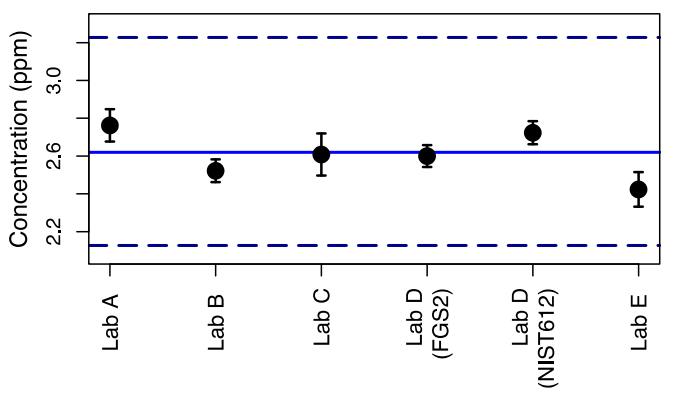

Nd146

K2 Inner vs Sample 201 Inner

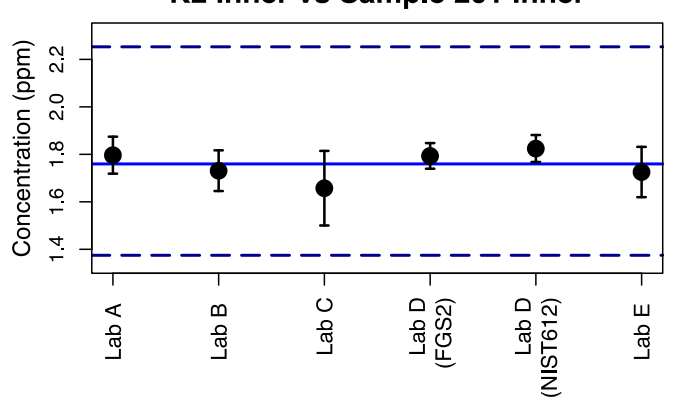

Sr88

K2 Inner vs Sample 201 Inner

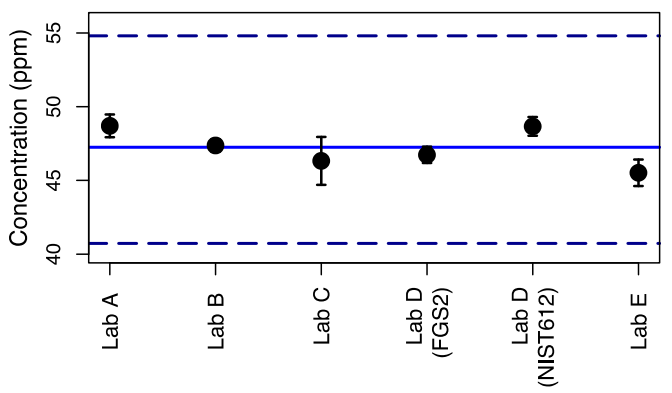

Ba137

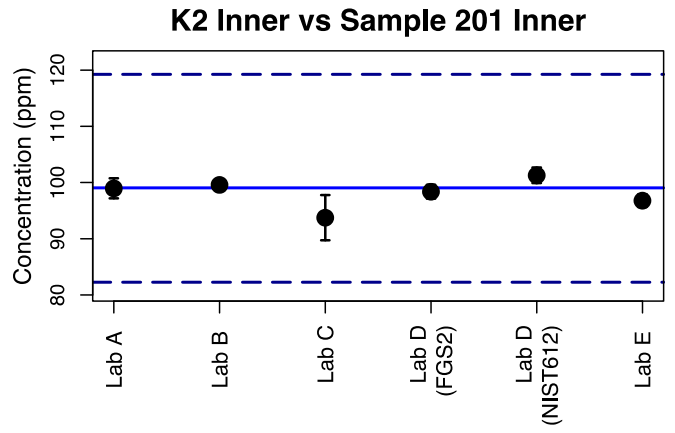

Ce140

K2 Inner vs Sample 201 Inner

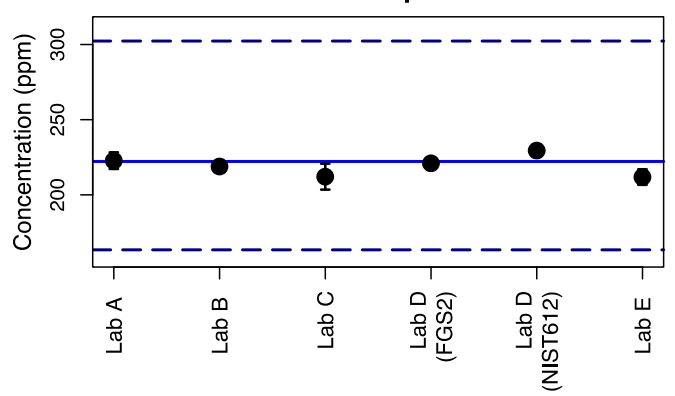

$\mathrm{Pb} 208$

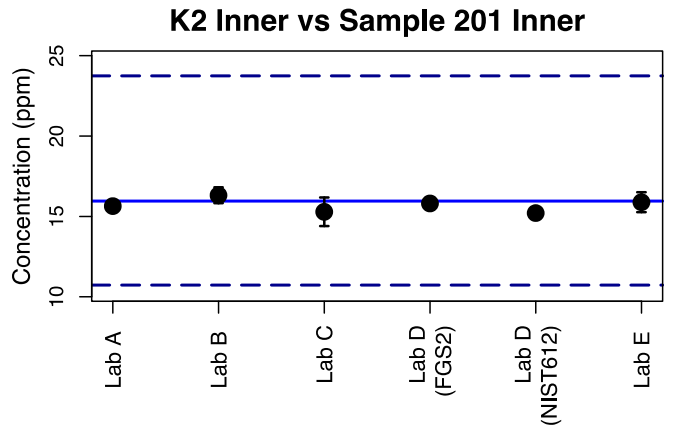


5. Comparison of Second Inter-laboratory K glasses to Duplicate

- K1 Inner vs. Sample 78 Inner

Li7

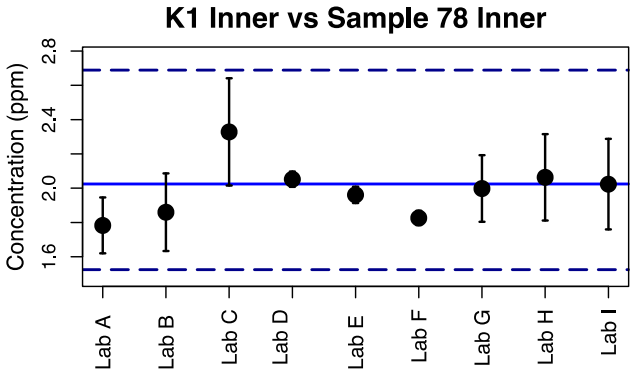

Al27

K1 Inner vs Sample 78 Inner

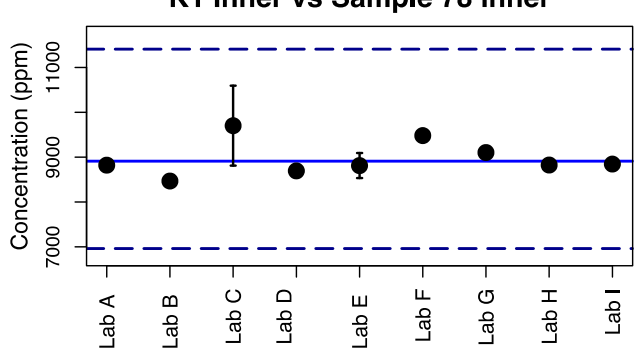

Ca42

K1 Inner vs Sample 78 Inner

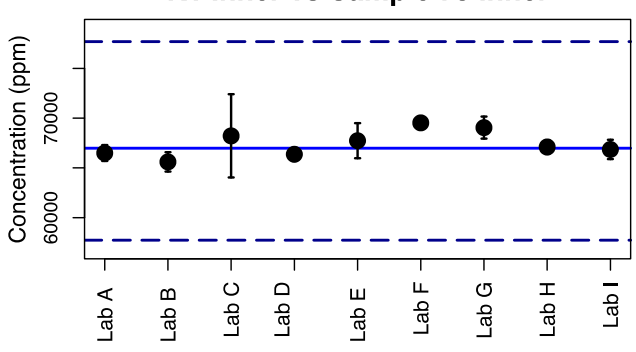

Mn55

K1 Inner vs Sample 78 Inner

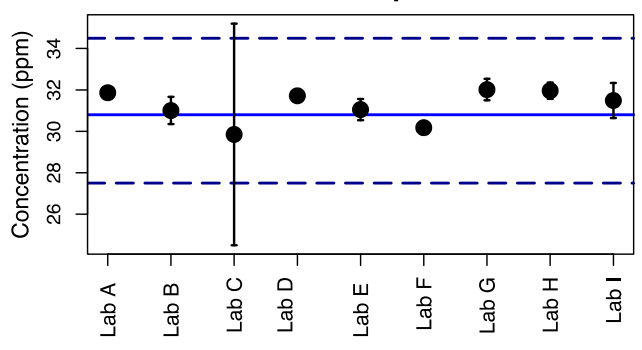

Mg25

K1 Inner vs Sample 78 Inner

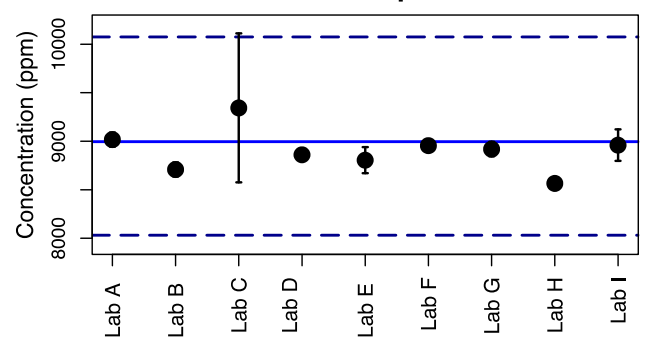

K39

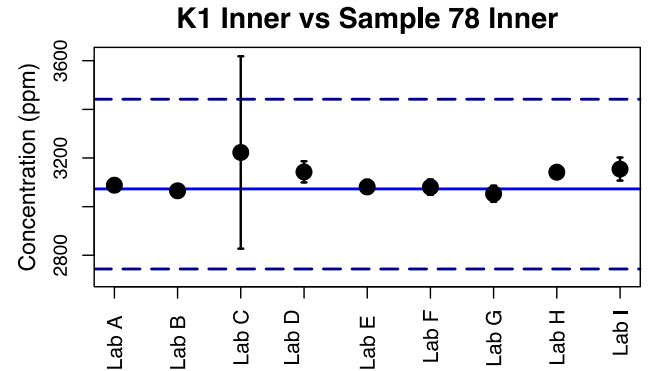

Ti49

K1 Inner vs Sample 78 Inner

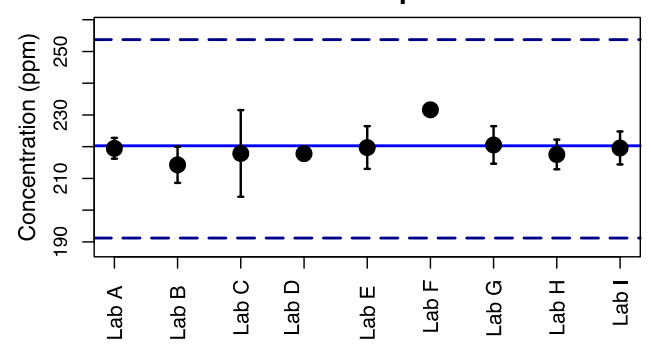

Fe57

K1 Inner vs Sample 78 Inner

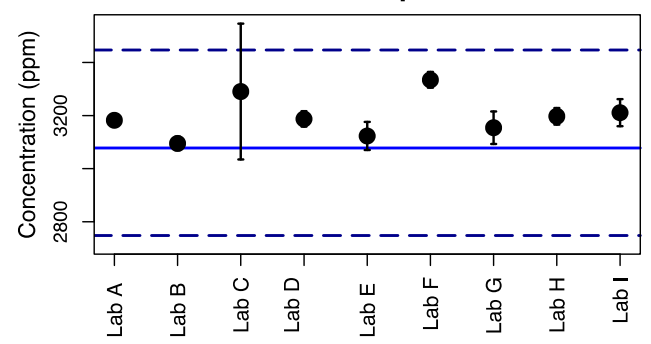


Rb85

K1 Inner vs Sample 78 Inner

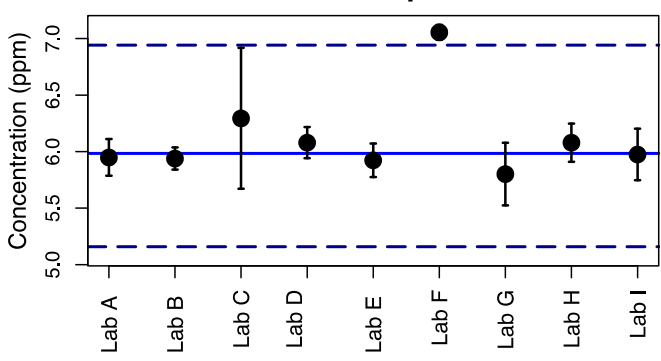

Zr90

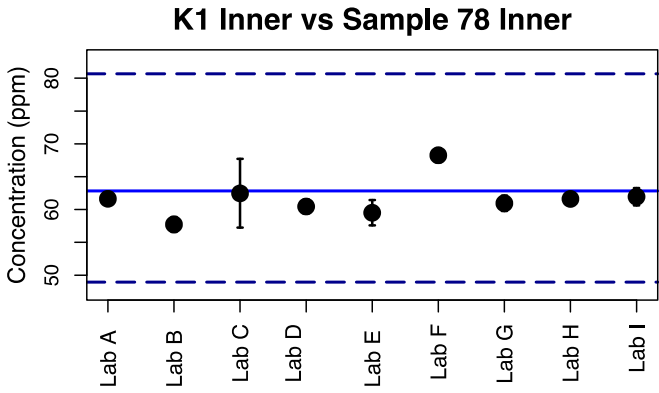

La139

K1 Inner vs Sample 78 Inner

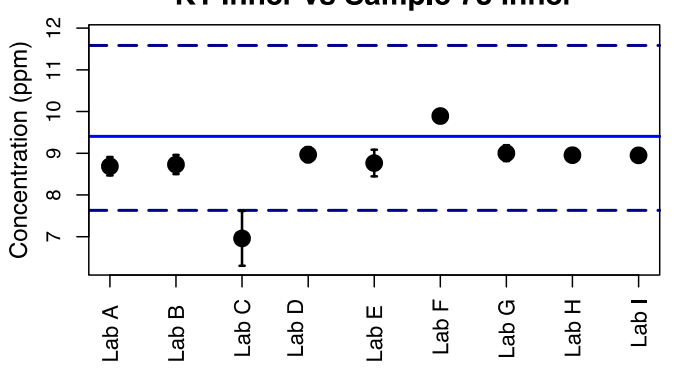

Nd146

K1 Inner vs Sample 78 Inner

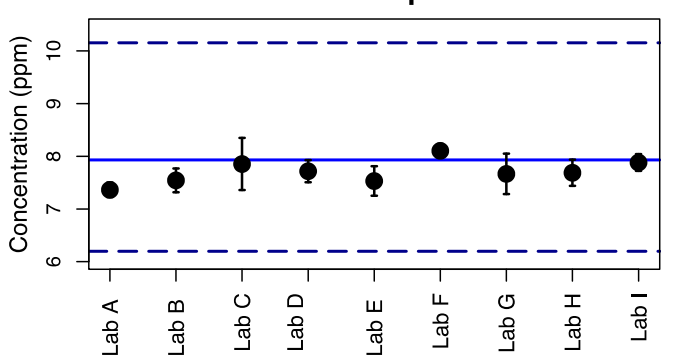

Sr88

K1 Inner vs Sample 78 Inner

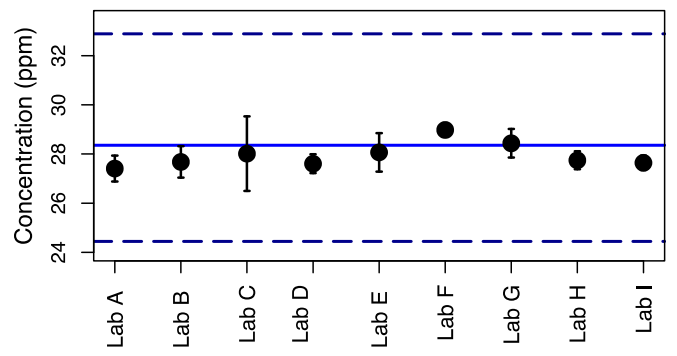

Ba137

K1 Inner vs Sample 78 Inner

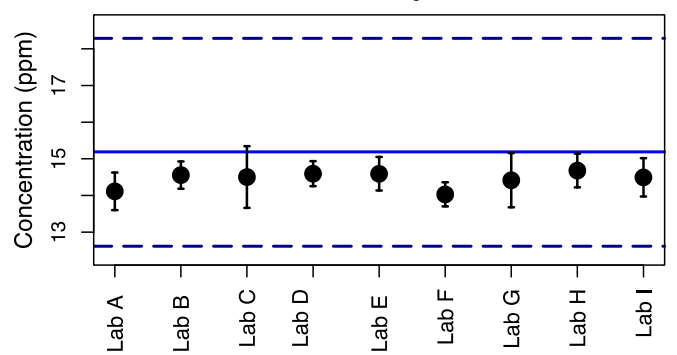

Ce140

K1 Inner vs Sample 78 Inner

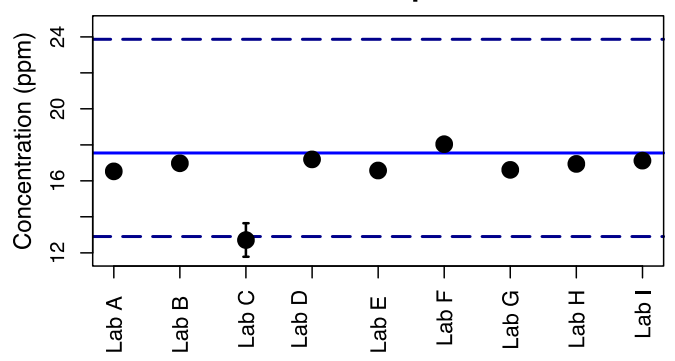

$\mathrm{Pb} 208$

K1 Inner vs Sample 78 Inner

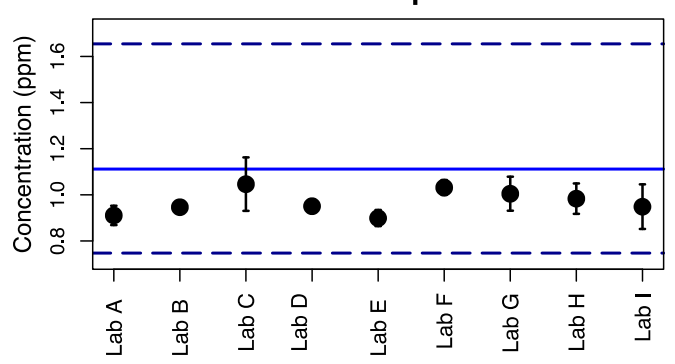


- K1 Outer vs. Sample 78 Outer

Li7

K1 Outer vs Sample 78 Outer

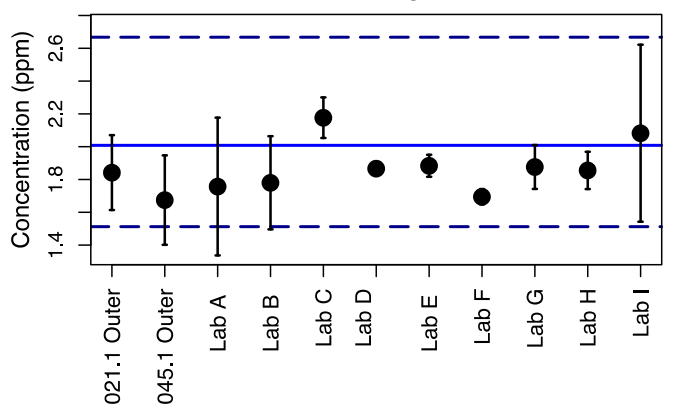

Al27

K1 Outer vs Sample 78 Outer

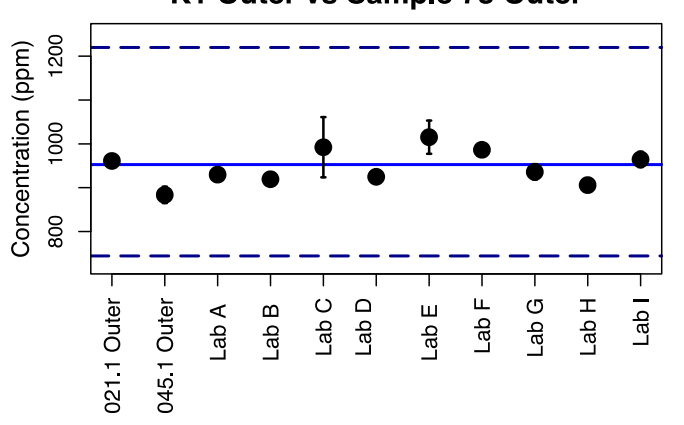

Ca42

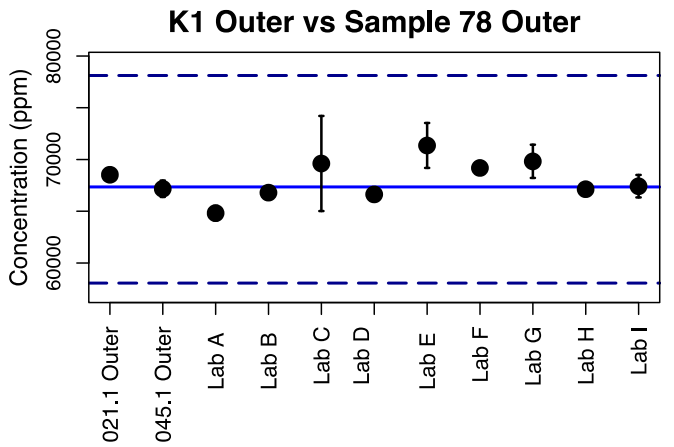

Mn55

K1 Outer vs Sample 78 Outer

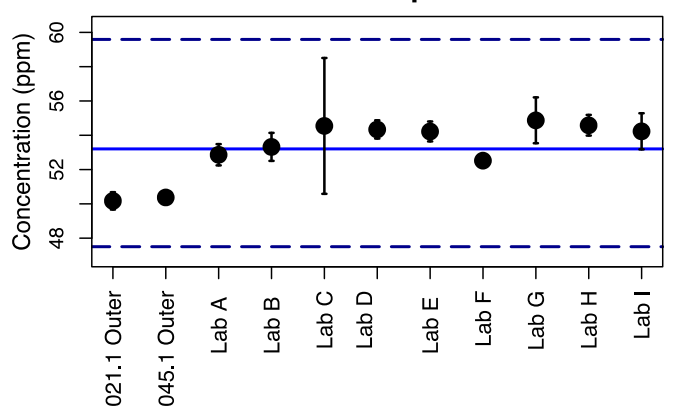

Mg25

K1 Outer vs Sample 78 Outer

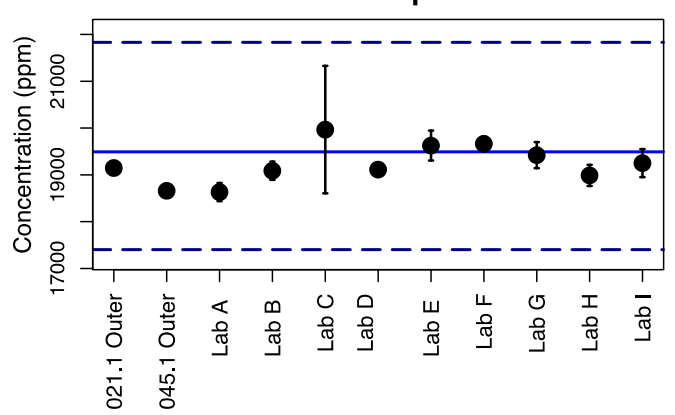

K39

K1 Outer vs Sample 78 Outer

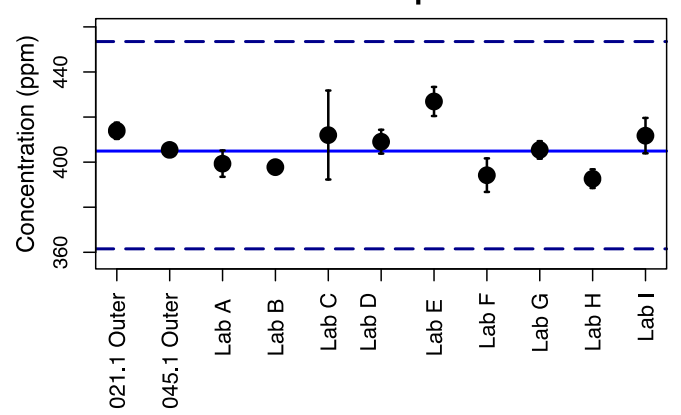

Ti49

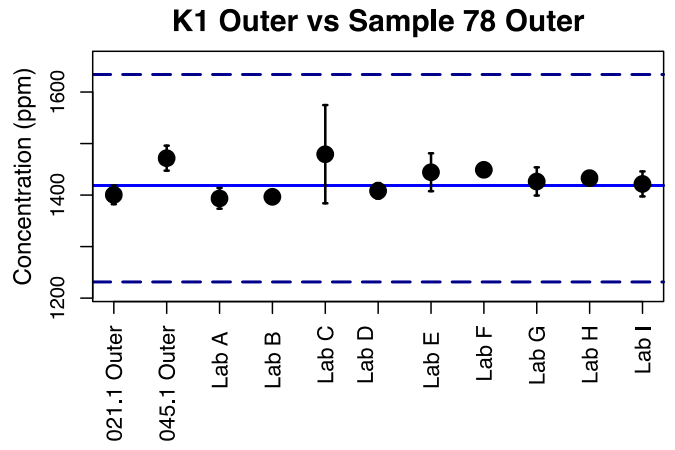

Fe57

K1 Outer vs Sample 78 Outer

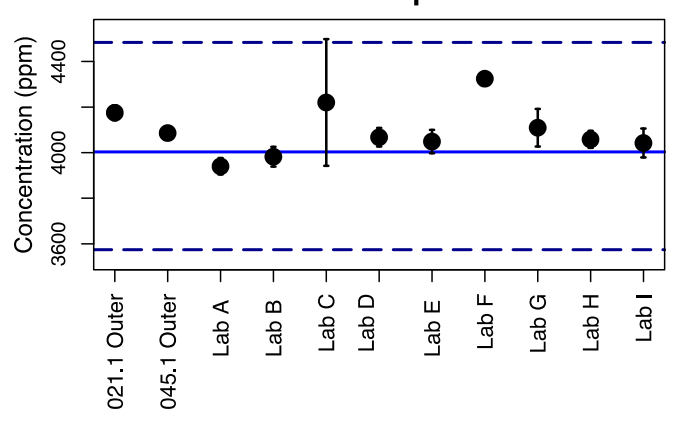


Rb85

K1 Outer vs Sample 78 Outer

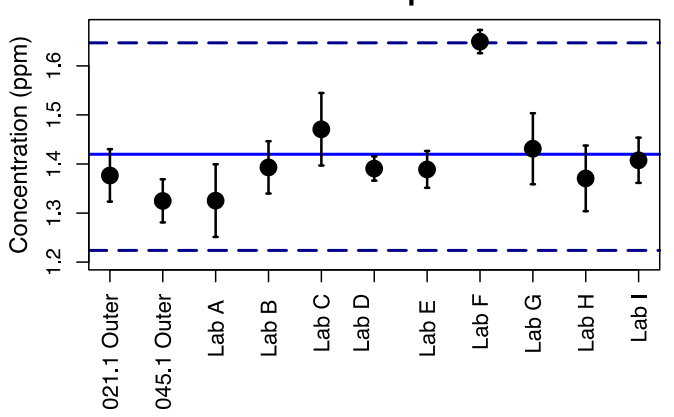

Zr90

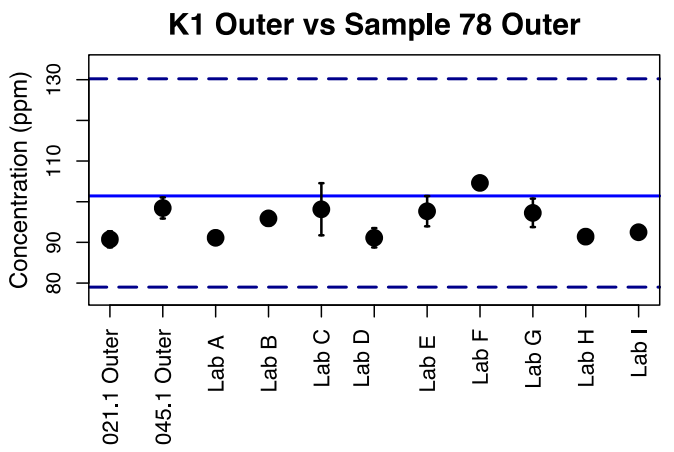

La139

K1 Outer vs Sample 78 Outer

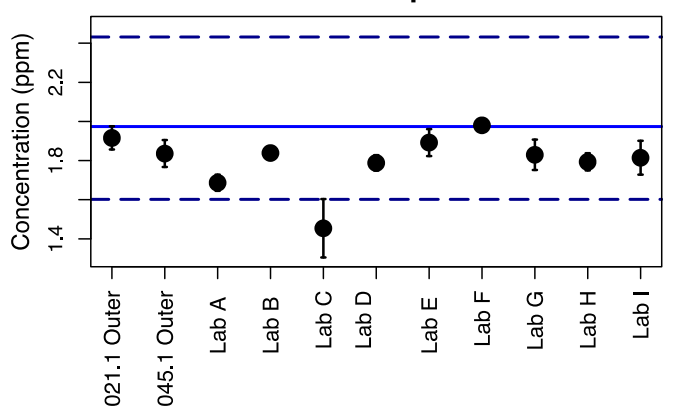

Nd146

K1 Outer vs Sample 78 Outer

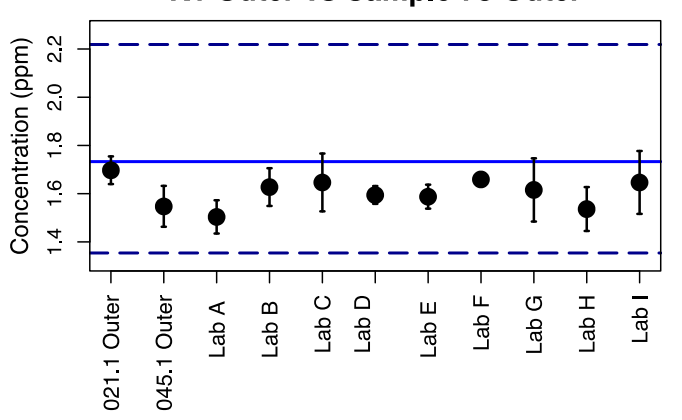

Sr88

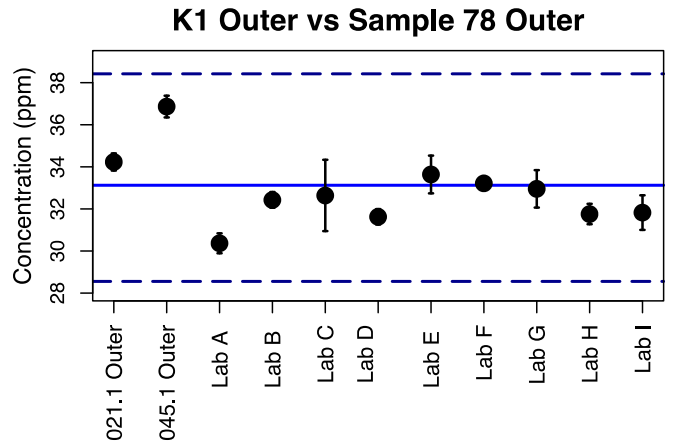

Ba137

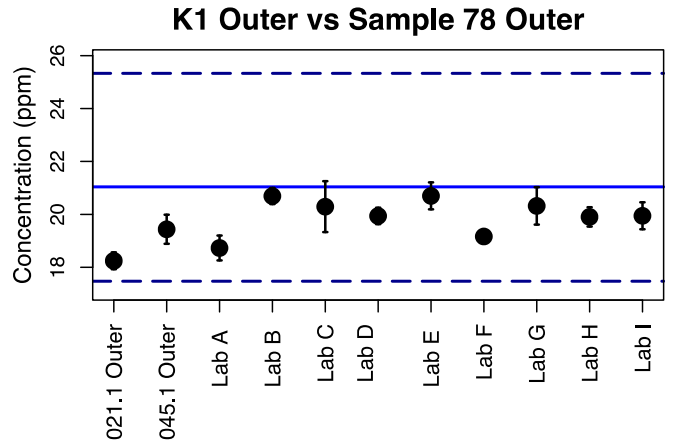

Ce140

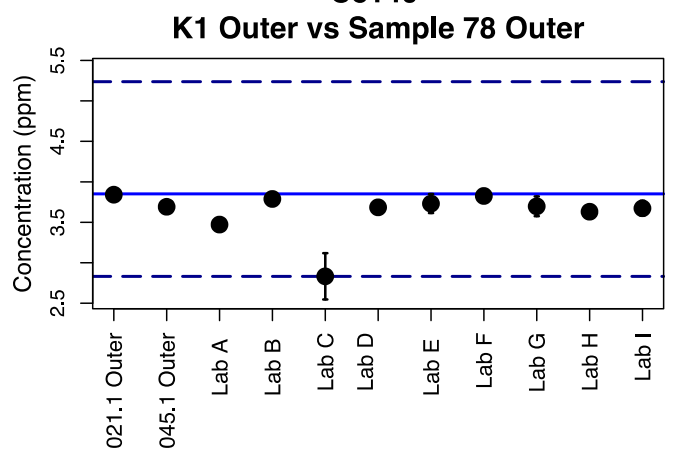

Pb208

K1 Outer vs Sample 78 Outer

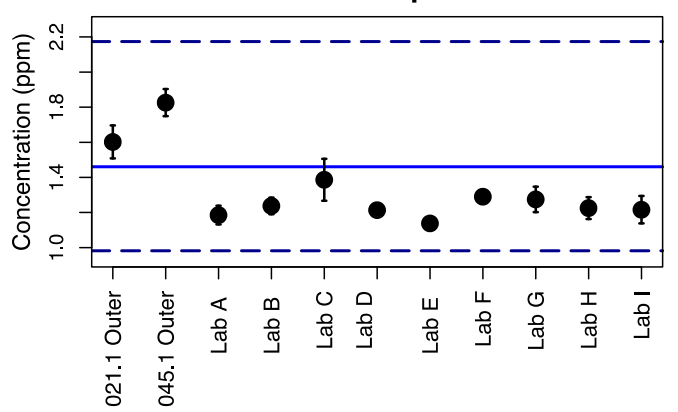


6. Comparison of Third Inter-laboratory $\mathrm{K}$ glasses to Duplicate

- K1 Inner vs. Sample 21

Li7

K1 Inner vs Sample 21 Inner

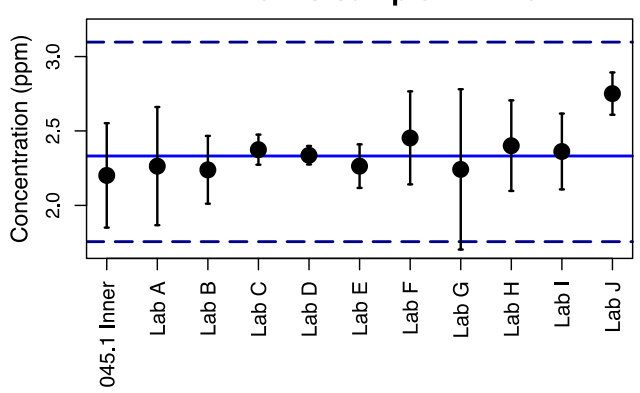

Al27

K1 Inner vs Sample 21 Inner

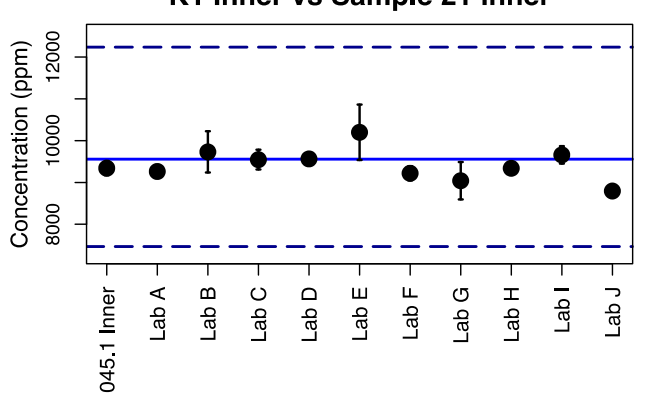

Ca42

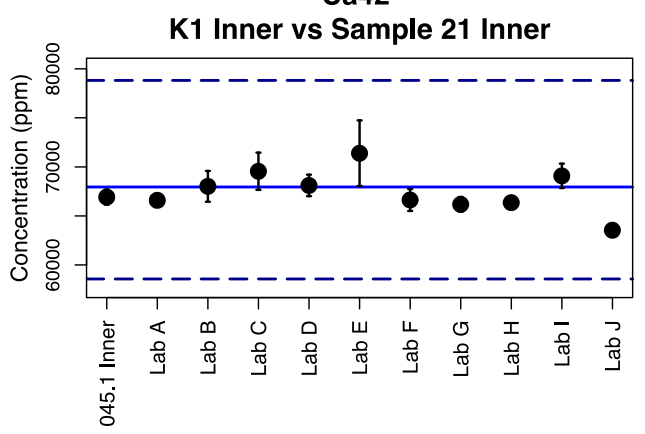

Mn55

K1 Inner vs Sample 21 Inner

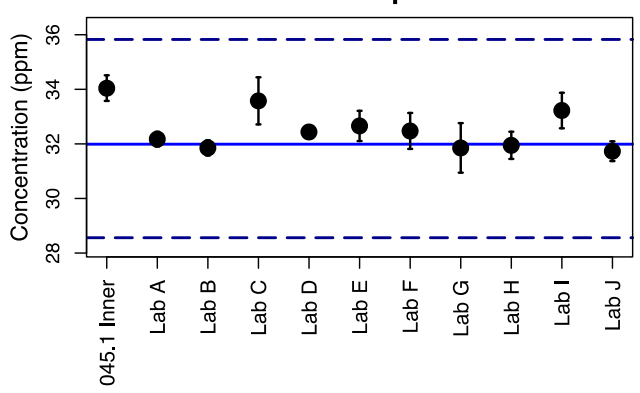

Mg25

K1 Inner vs Sample 21 Inner

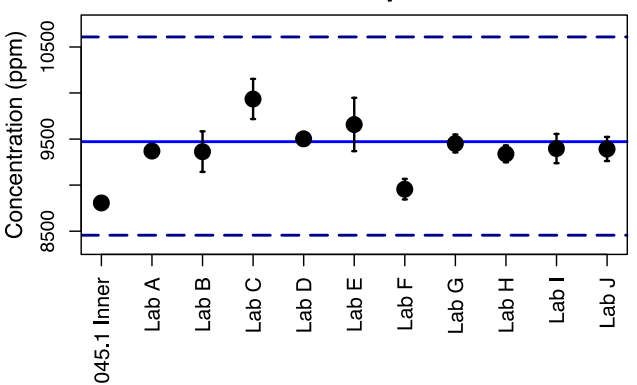

K39

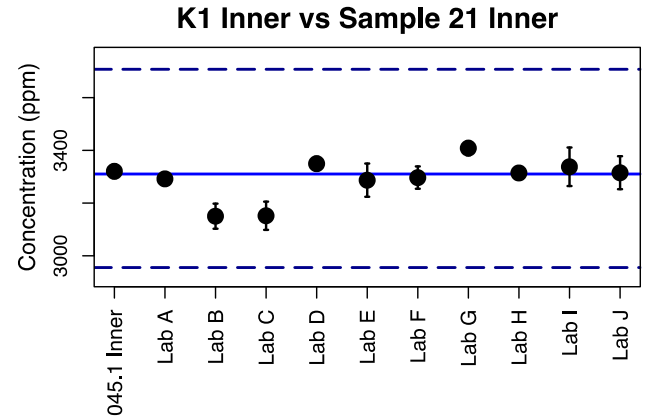

Ti49

K1 Inner vs Sample 21 Inner

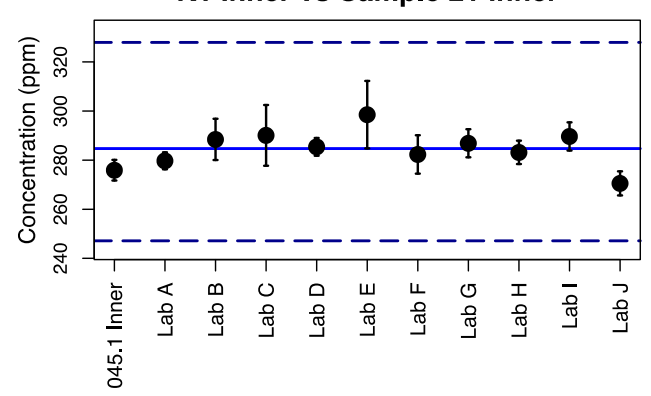

Fe57

K1 Inner vs Sample 21 Inner

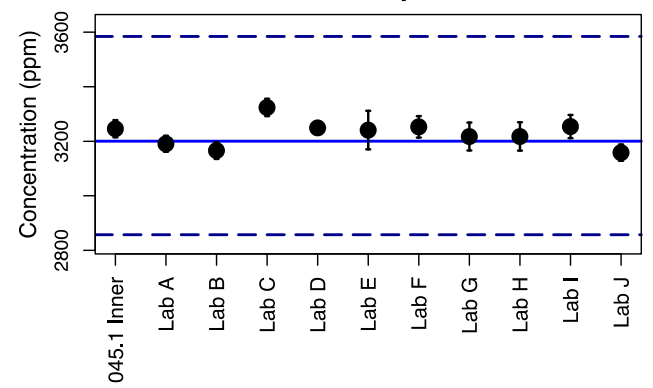


Rb85

K1 Inner vs Sample 21 Inner

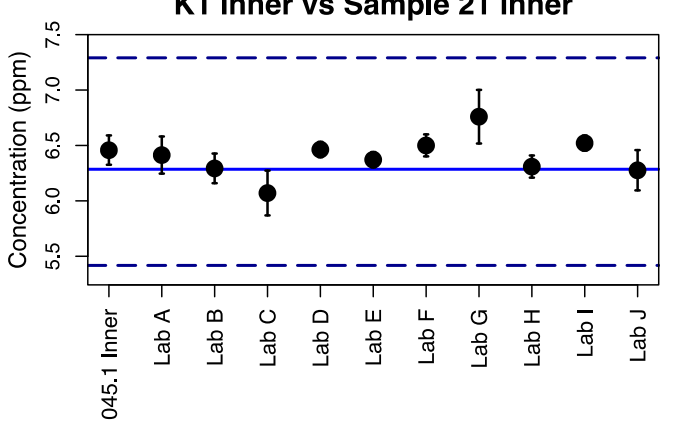

Zr90

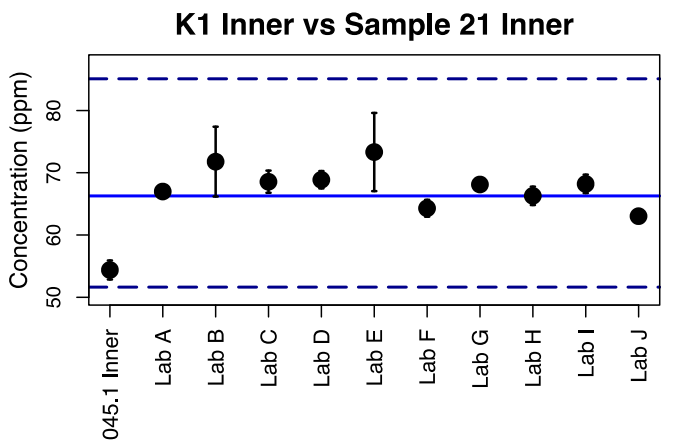

La139

K1 Inner vs Sample 21 Inner

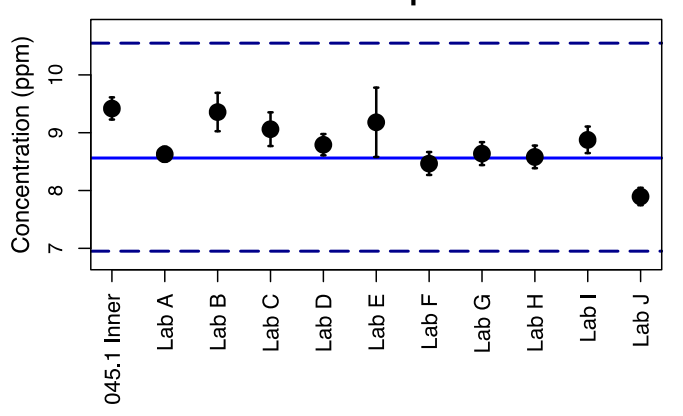

Nd146

K1 Inner vs Sample 21 Inner

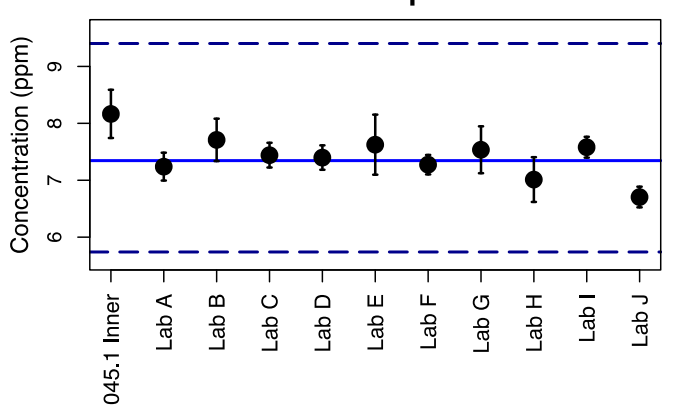

Sr88

K1 Inner vs Sample 21 Inner

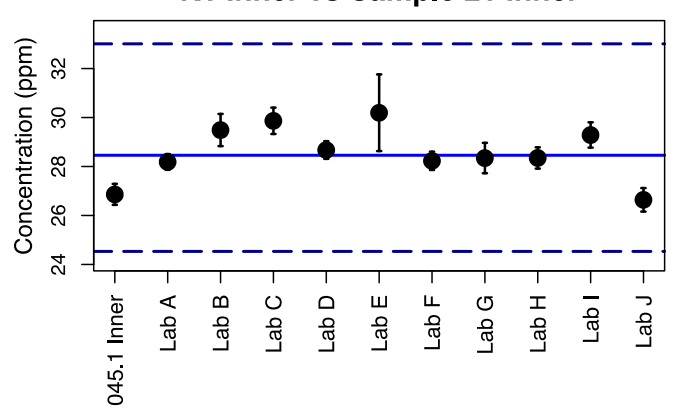

Ba137

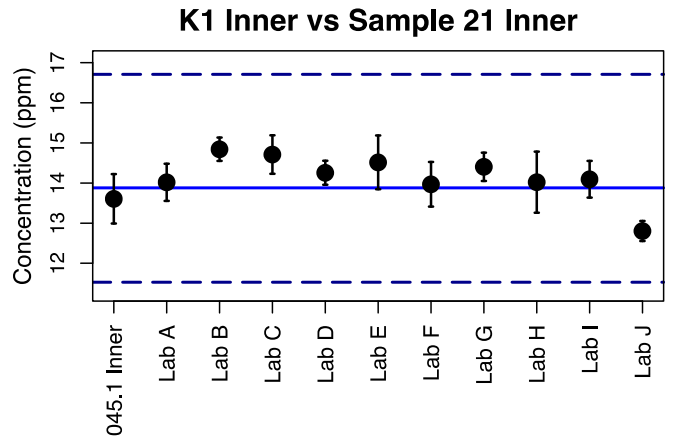

Ce140

K1 Inner vs Sample 21 Inner

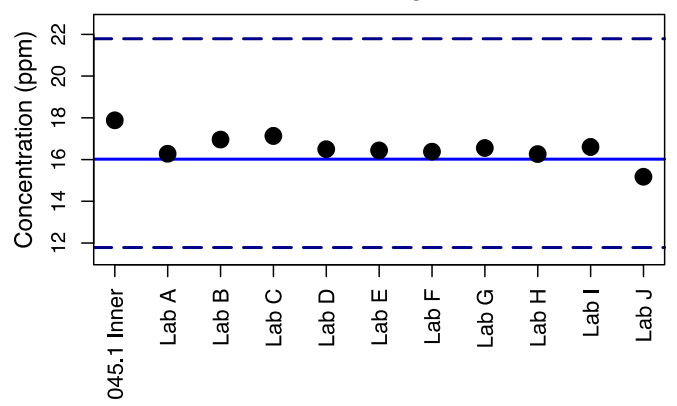

Pb208

K1 Inner vs Sample 21 Inner

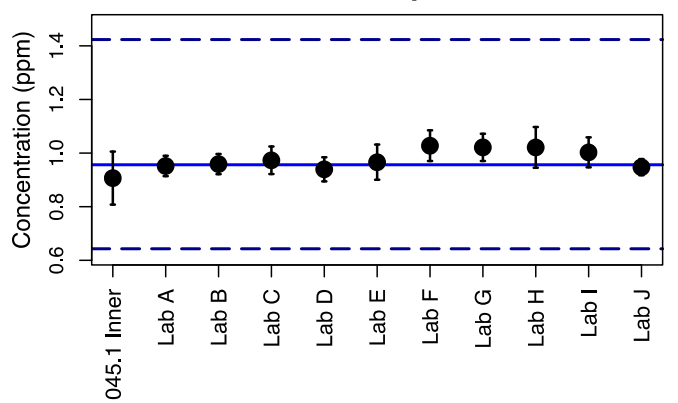


- K1 Outer vs. Sample 21 Outer

Li7

K1 Outer vs Sample 21 Outer

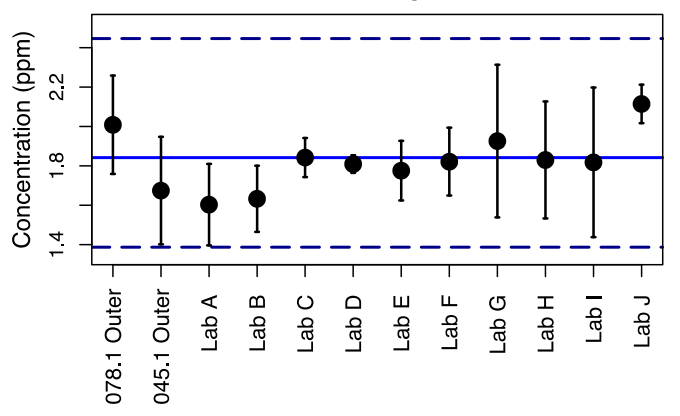

Al27

K1 Outer vs Sample 21 Outer

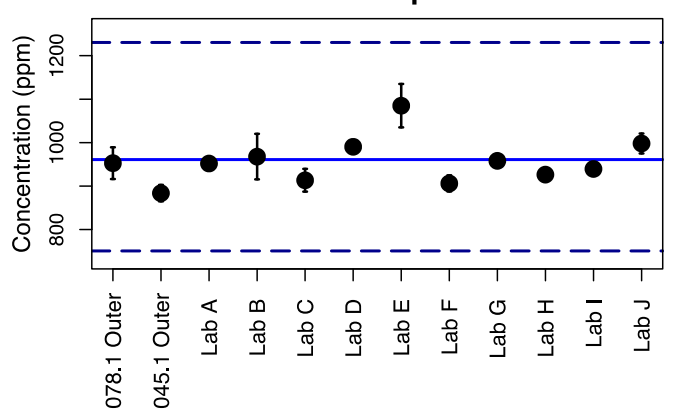

Ca42

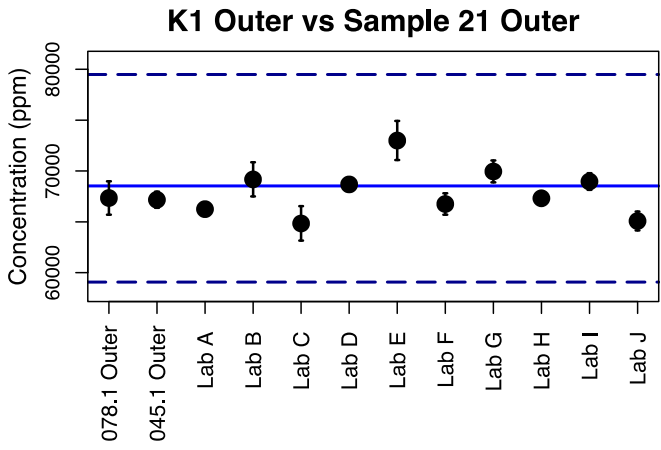

Mn55

K1 Outer vs Sample 21 Outer

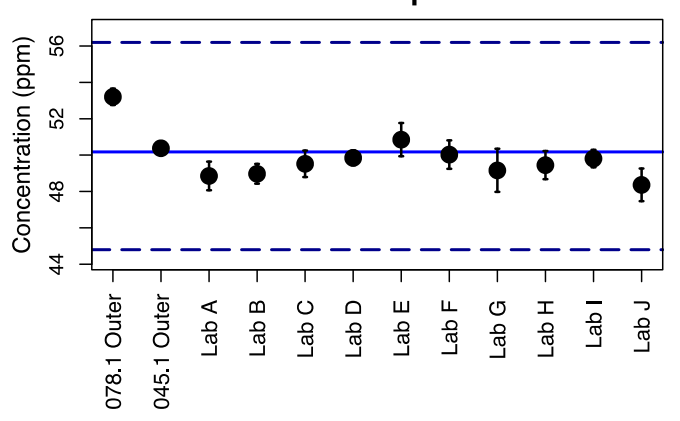

Mg25

K1 Outer vs Sample 21 Outer

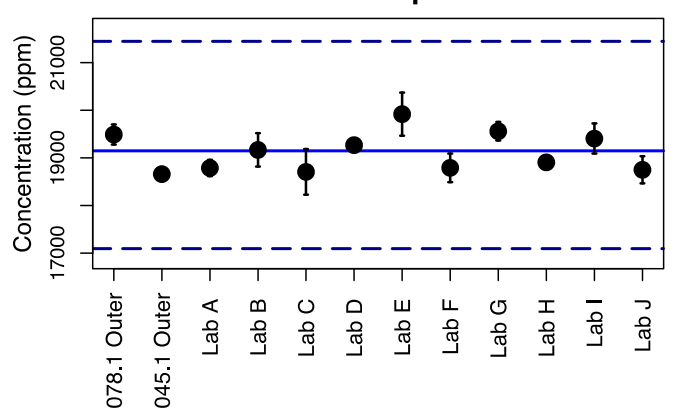

K39

K1 Outer vs Sample 21 Outer

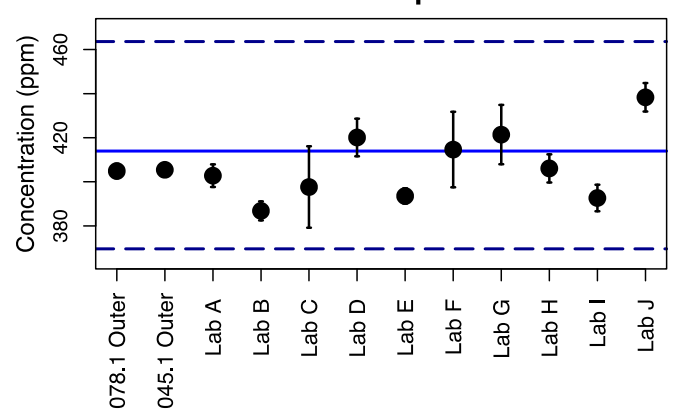

Ti49

K1 Outer vs Sample 21 Outer

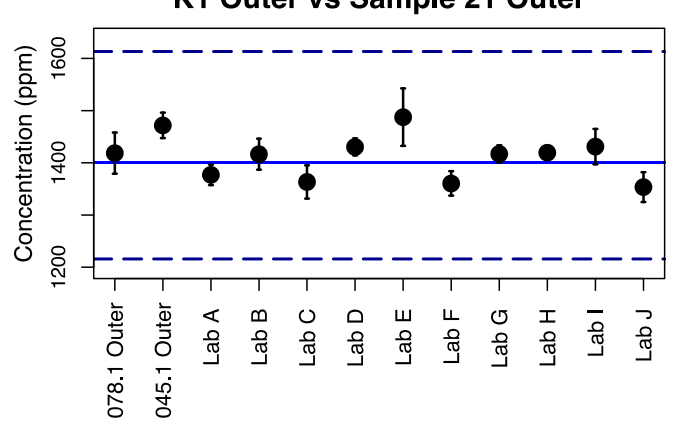

Fe57

K1 Outer vs Sample 21 Outer

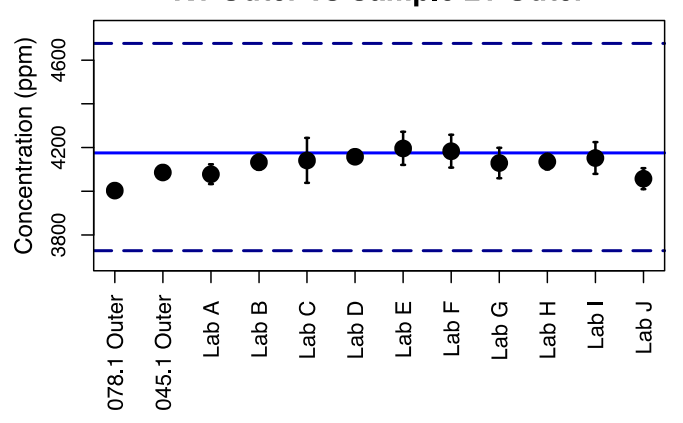


Rb85

K1 Outer vs Sample 21 Outer

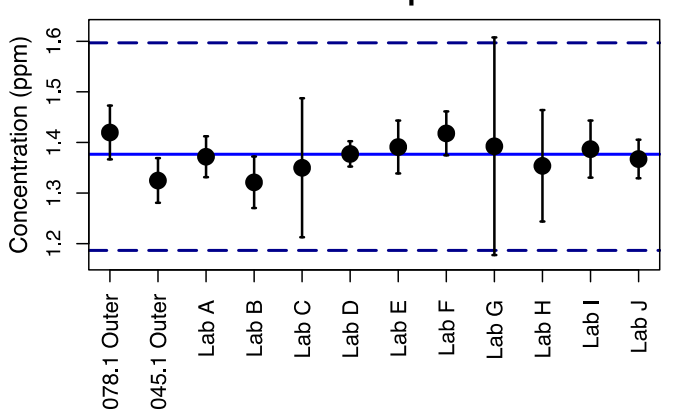

Zr90

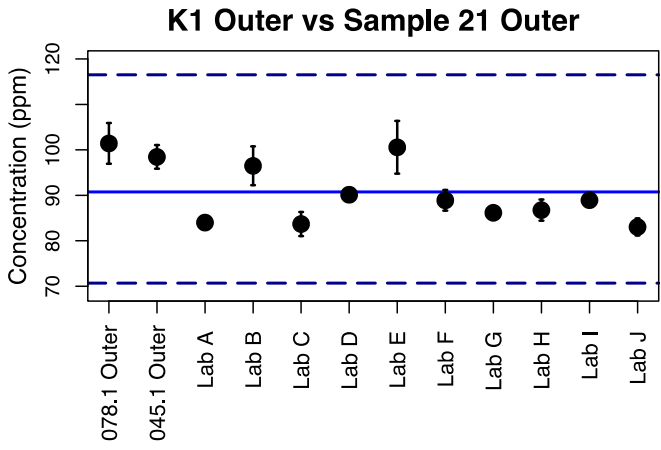

La139

K1 Outer vs Sample 21 Outer

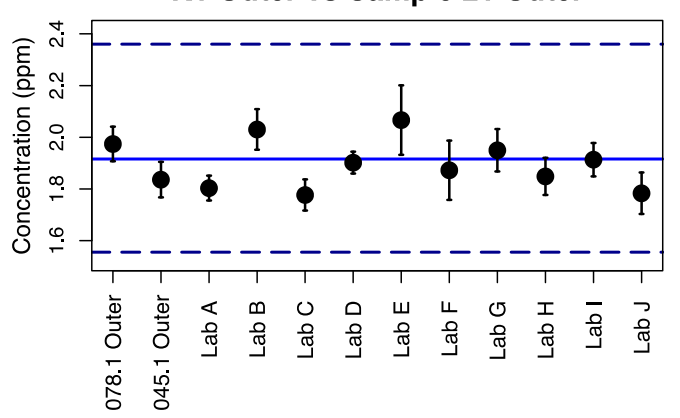

Nd146

K1 Outer vs Sample 21 Outer

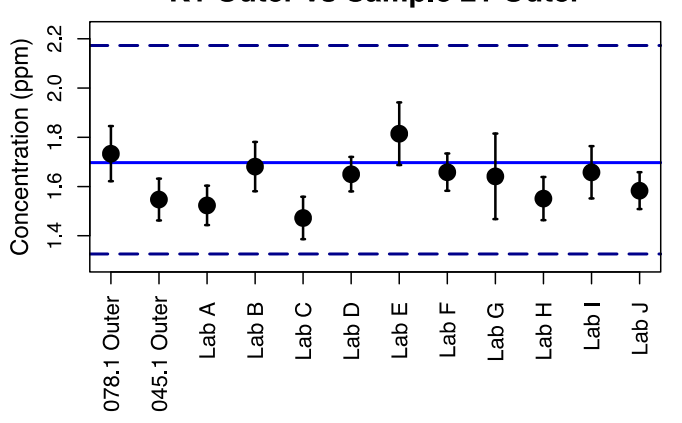

Sr88

K1 Outer vs Sample 21 Outer

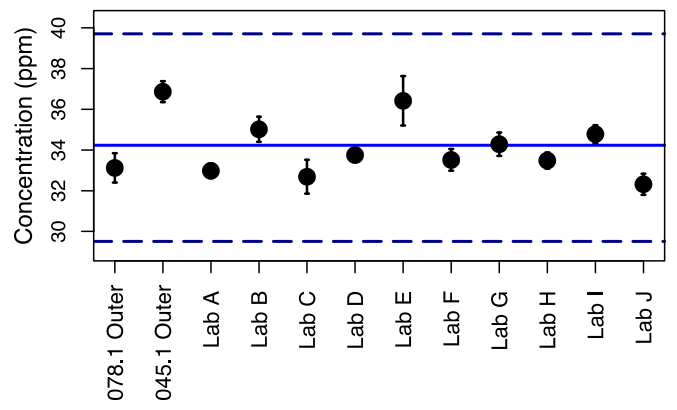

Ba137

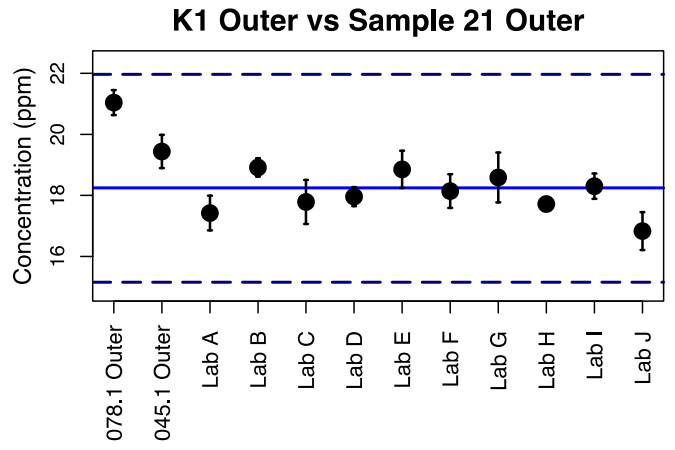

Ce140

K1 Outer vs Sample 21 Outer

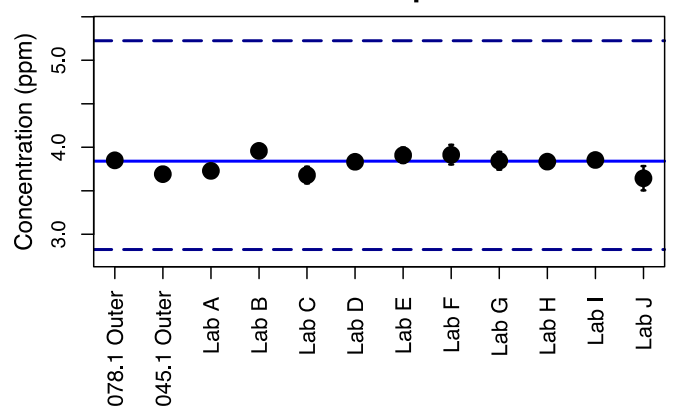

Pb208

K1 Outer vs Sample 21 Outer

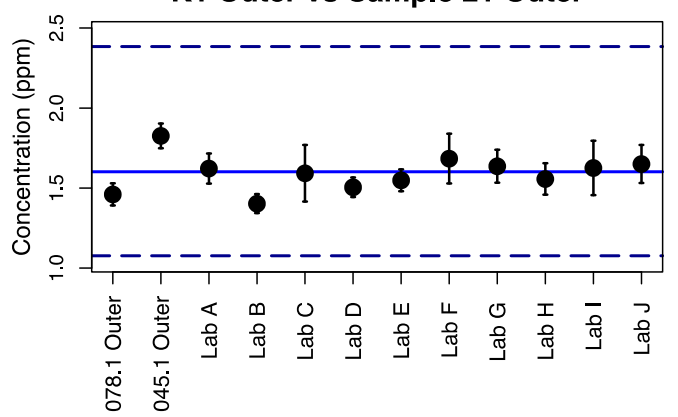


7. Inter-laboratory Quality Control Glasses NIST1831 and FGS1

- First Inter-Laboratory Study NIST1831
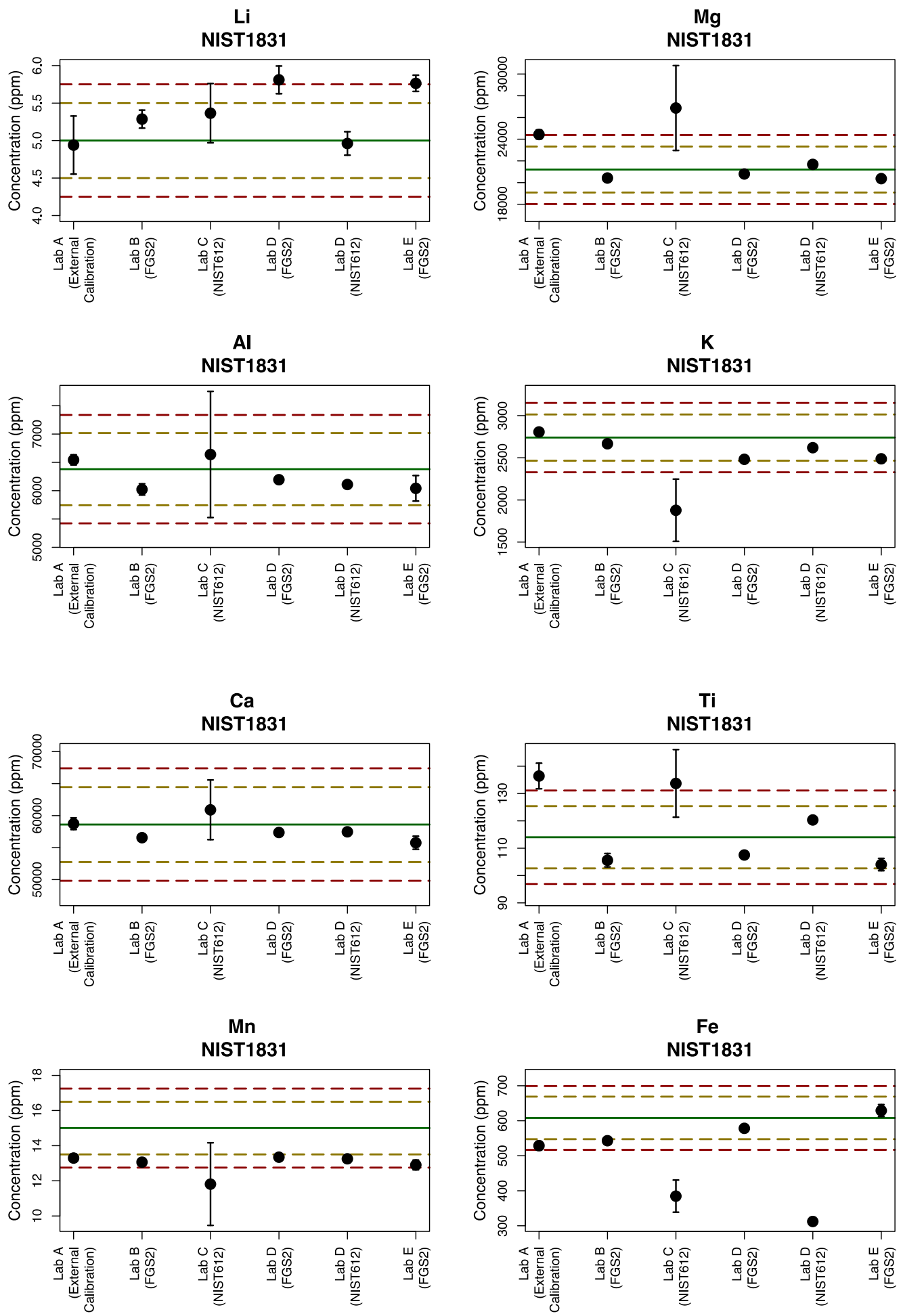

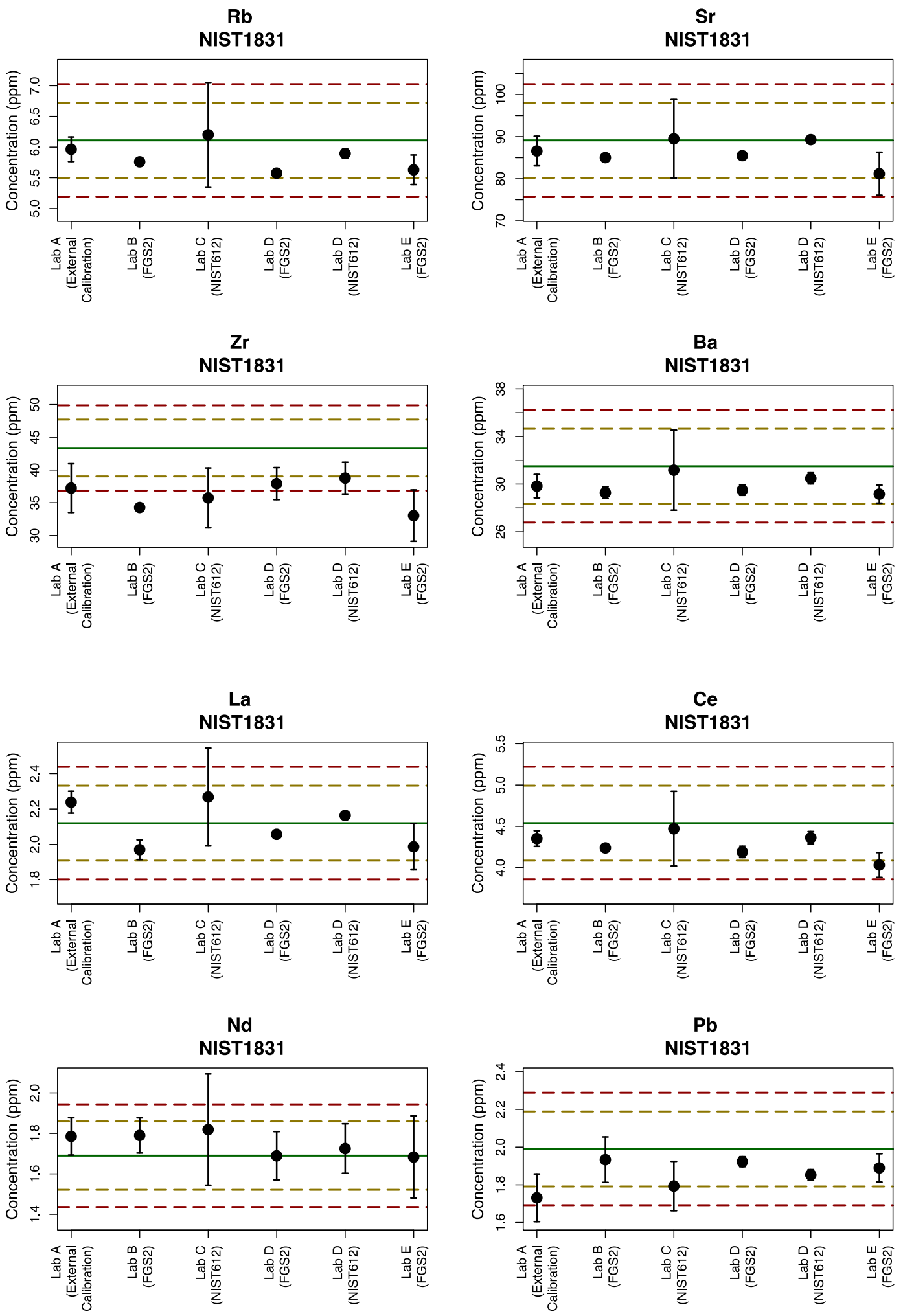
- Second Inter-Laboratory Study FGS1
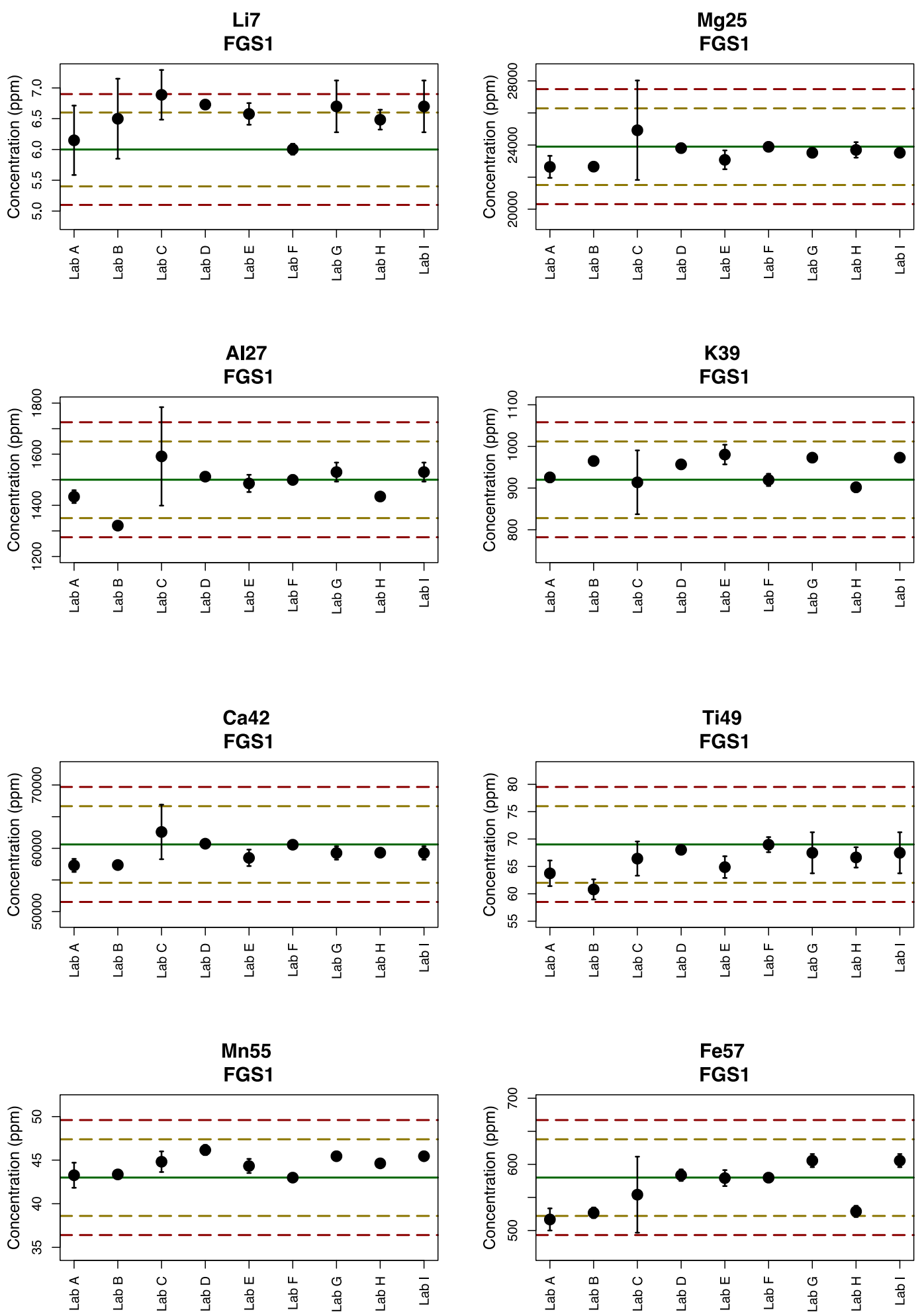

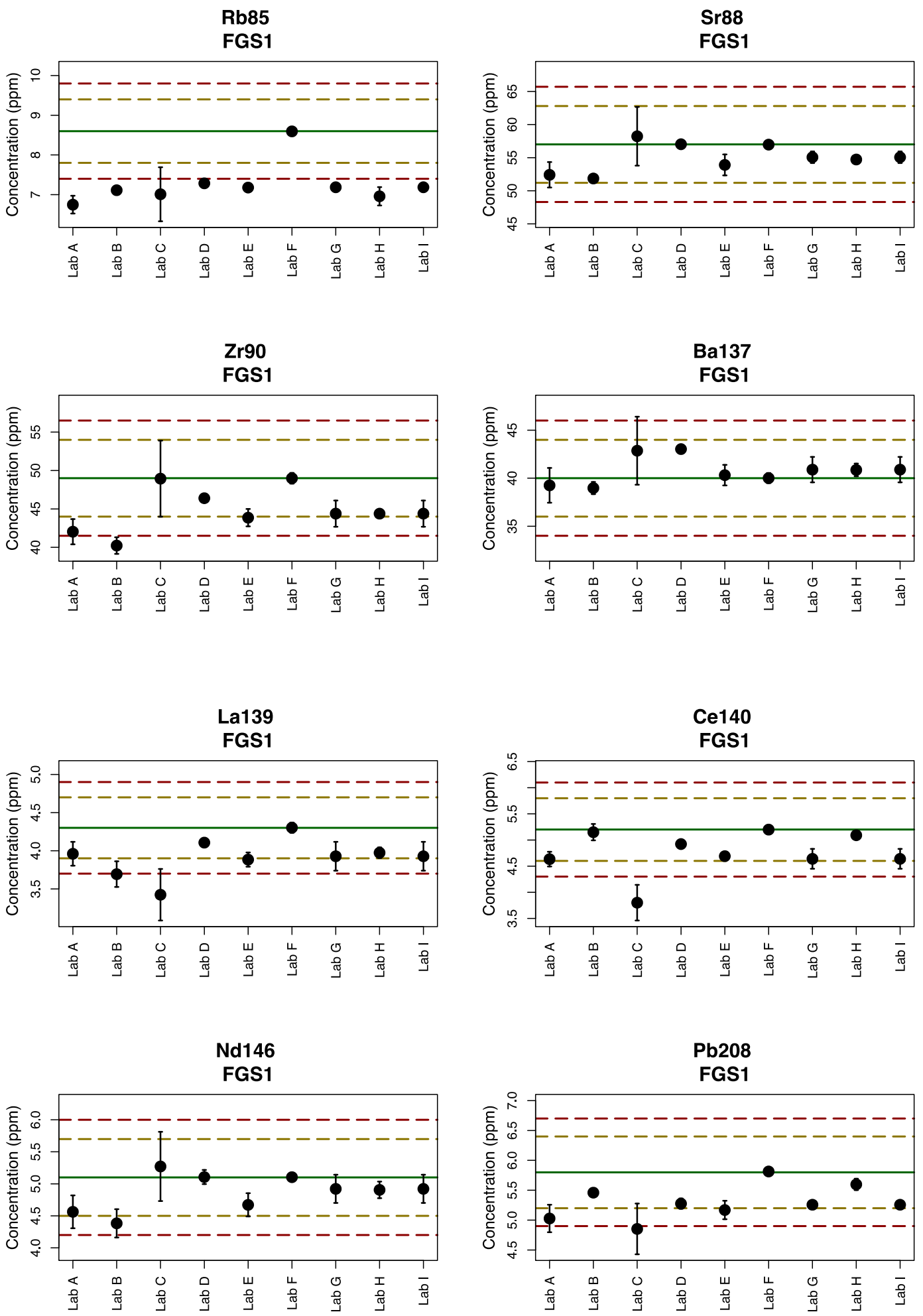
- Third Inter-Laboratory Study FGS1
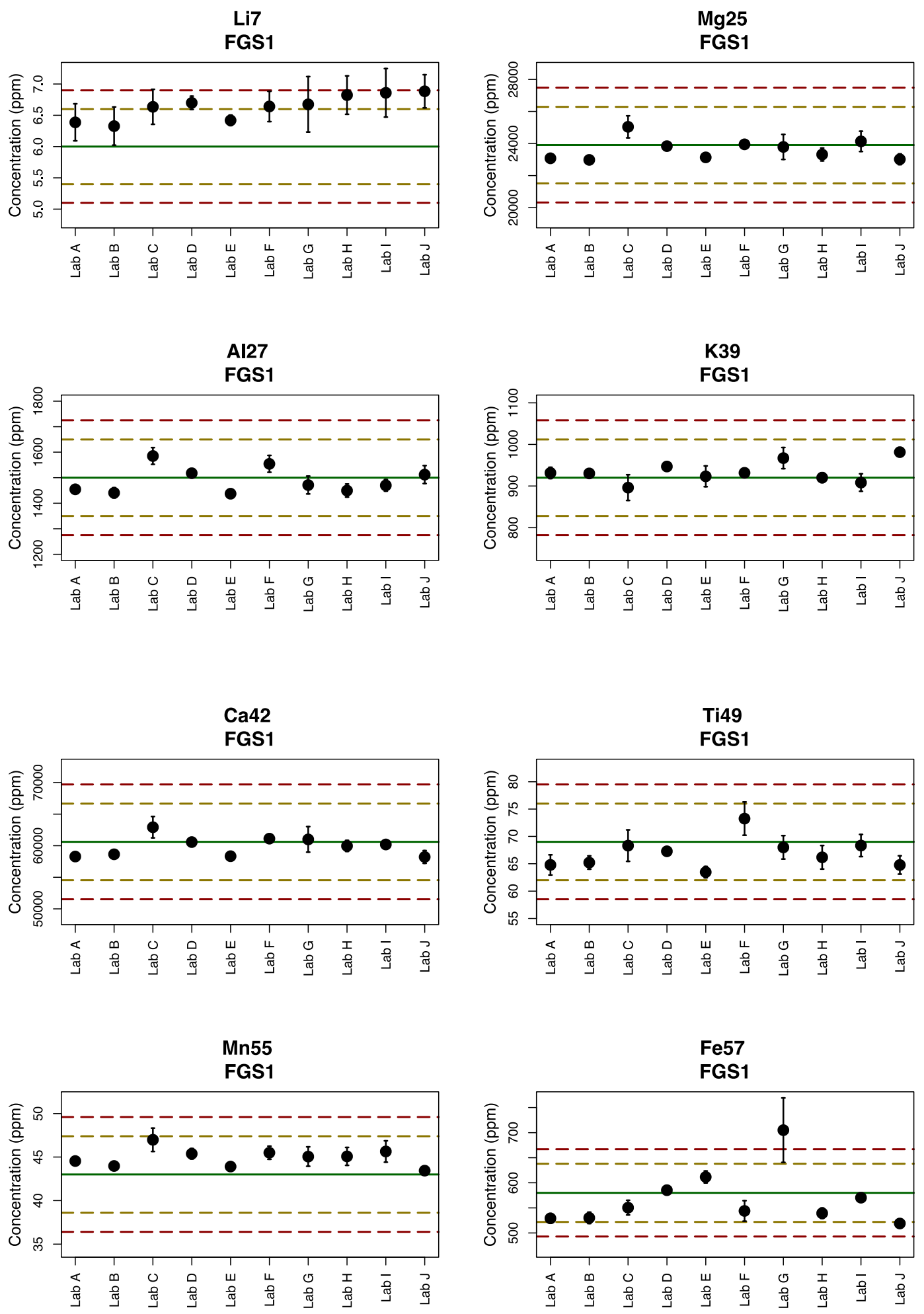
Rb85

FGS1

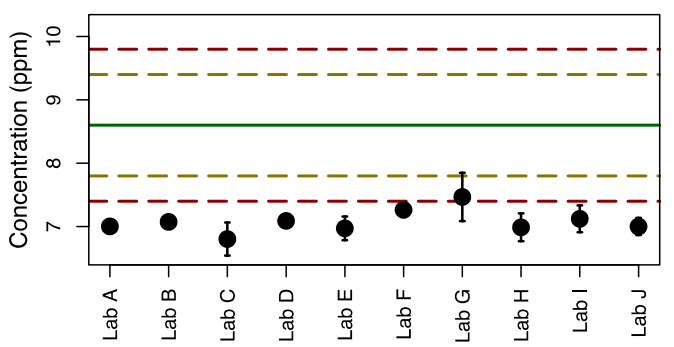

Zr90

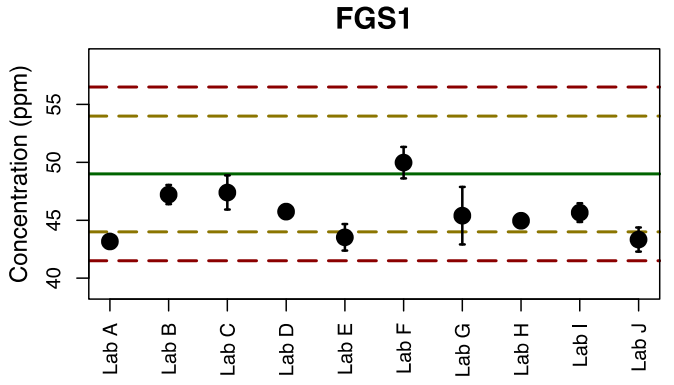

Sr88

FGS1

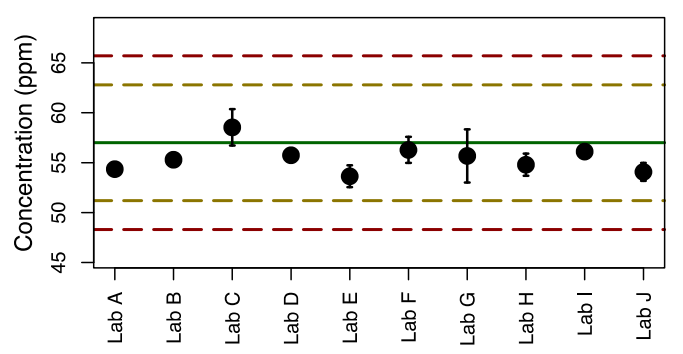

Ba137

FGS1

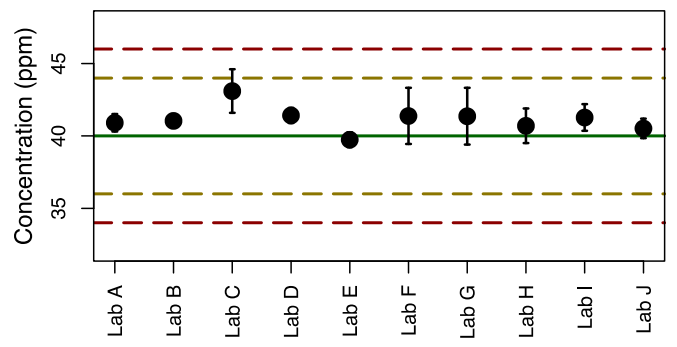

Ce140

FGS1

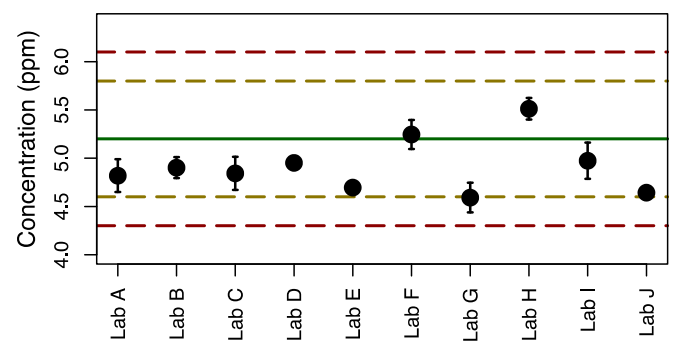

Pb208

FGS1

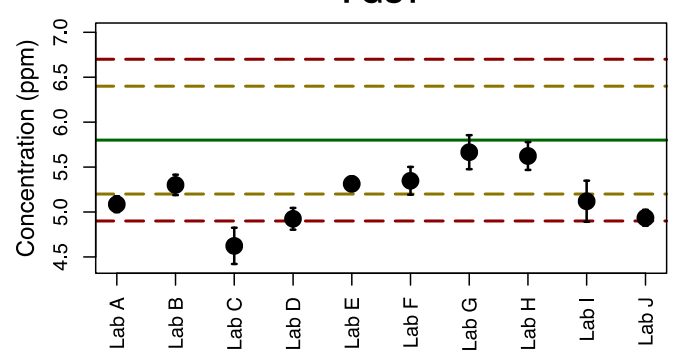


VITA

TRICIA MARIE HOFFMAN

Born, Saint Marys, PA

2009-2013 B.S., Chemistry

Edinboro University of Pennsylvania

Edinboro, PA

2013-2016 M.S., Chemistry

Florida International University

Miami, FL

2015-2017

Nuclear Regulatory Commission Fellow

2016-2018 Doctoral Candidate, Chemistry

Florida International University

Miami, FL

\section{PUBLICATIONS AND PRESENTATIONS}

R. Corzo, T. Hoffman, P. Weis, J. Franco-Pedroso, D. Ramos, J. Almirall. The Use of LA-ICP-MS Databases to Estimate Likelihood Ratios for the Forensic Analysis of Glass Evidence. Talanta 2018.

T. Hoffman, R. Jaćimović, L. J. Bay, J. Griboff, M. Jagodic, M. Monferrán, N. Ogrinc, I. Podkolzin, D. Wunderlin, J. Almirall. Development of a Method for the Elemental Analysis of Milk Powders Using Laser Ablation-Inductively Coupled Plasma-Mass Spectrometry (LA-ICP-MS) and its Potential Use in Geographic Sourcing. Talanta 2018.

T. Hoffman, R. Corzo, P. Weis, E. Pollock, A. van Es, W. Wiarda, A. Stryjnik, H. Dorn, A. Heydon, E. Hoise, S. Le Franc, X. Huifang, B. Pena, T. Scholz, J. Gonzalez, J. Almirall. An Inter-Laboratory Evaluation of LA-ICP-MS Analysis of Glass and the Use of a Database for the Interpretation of Glass Evidence. Forensic Chemistry (Submitted).

T. Hoffman, J. Almirall, Elemental analysis of milk powders using laser-based methods, Third Research Coordination Meeting on the Accessible Technologies for the Verification of Origin of Dairy Products as an Example Control System to Enhance Global Trade and Food Safety, October 2017, Vienna, Austria. 
T. Hoffman, R. Corzo, J. R. Almirall. Glass Interpretation Working Group: Results from Two Inter-laboratory Exercises. 23rd Annual EPG Meeting, September 2017, Athens, Greece.

R. Corzo, T. Hoffman, J. R. Almirall. Development of a LIBS Database for the Forensic Interpretation of Glass Evidence. First Industry Advisory Board Meeting, August 2017, Miami, Florida, USA.

T. Hoffman, R. Corzo, J. R. Almirall. Strengthening the Significance of Glass Evidence through Inter-laboratory Tests: Design and Implementation of FIU Working Groups. NIST 2nd Colloquium on the Weight of Evidence, June 2017, Gaithersburg, Maryland, USA.

T. Hoffman, J. Almirall, Quantitative analysis of milk powders using laser-based methods, Accessible Technologies for the Verification of Origin of Dairy Products as an Example Control System to Enhance Global Trade and Food Safety, October 2016, Rabat, Morocco.

J. Almirall, T. Hoffman, R. Corzo, The state of data in glass analysis, NIST Trace Evidence Data Workshop, July 2016, Gaithersburg, Maryland, USA.

J.Almirall, T. Hoffman, Novel forensic applications using LA-ICP-MS and LIBS, IAEA CRP planning meeting, April 2016, Vienna, Austria.

T. Hoffman, T. Trejos, J. Almirall, Strengthening the evaluation and interpretation of glass evidence using statistical analysis of collection sets of refractive index and elemental data, AAFS, February 2016, Las Vegas, Nevada, USA (poster).

T. Hoffman, J. Almirall, Geographic sourcing and traceability of milk powder using chemical methods, US Dairy Export, May 2015, Washington DC, USA.

J. Almirall, T. Hoffman, Novel forensic applications using LA-ICP-MS and LIBS, SCIX, September 2015, Providence, Rhode Island, USA. 